\title{
Seven-point conformal blocks in the extended snowflake channel and beyond
}

\author{
Jean-François Fortin, ${ }^{1, *}$ Wen-Jie Ma๑ ${ }^{1, \dagger}$ and Witold Skiba $\odot^{2, \$}$ \\ ${ }^{1}$ Département de Physique, de Génie Physique et d'Optique Université Laval, \\ Québec, QC GIV 0A6, Canada \\ ${ }^{2}$ Department of Physics, Yale University, New Haven, Connecticut 06520, USA
}

(Received 1 September 2020; accepted 21 October 2020; published 1 December 2020)

\begin{abstract}
Seven-point functions have two inequivalent topologies or channels. The comb channel has been computed previously, and here we compute scalar conformal blocks in the extended snowflake channel in $d$ dimensions. Our computation relies on the known action of the differential operator that sets up the operator product expansion in embedding space. The scalar conformal blocks in the extended snowflake channel are obtained as a power series expansion in the conformal cross-ratios whose coefficients are a triple sum of the hypergeometric type. This triple sum factorizes into a single sum and a double sum. The single sum can be seen as originating from the comb channel and is given in terms of $\mathrm{a}_{3} F_{2}$-hypergeometric function, while the double sum originates from the snowflake channel which corresponds to a Kampé de Fériet function. We verify that our results satisfy the symmetry properties of the extended snowflake topology. Moreover, we check that the behavior of the extended snowflake conformal blocks under several limits is consistent with known results. Finally, we conjecture rules leading to a partial construction of scalar $M$-point conformal blocks in arbitrary topologies.
\end{abstract}

DOI: $10.1103 /$ PhysRevD.102.125007

\section{INTRODUCTION}

With their extended spacetime symmetry group, conformal field theories (CFTs) are possibly amenable to exact nonperturbative solutions. Indeed, from the operator product expansion (OPE), which expresses the product of two quasiprimary operators in terms of an infinite sum of quasiprimary operators and their descendants, conformal correlation functions can be expanded in terms of the CFT data (the OPE coefficients and operator dimensions, which encode in particular the spectrum of quasiprimary operators of the CFT and their couplings) and conformal blocks. The blocks are functions of the conformal cross-ratios (scalar quantities built from the spacetime coordinates that are invariant under the conformal group) and are completely determined by conformal covariance, although it is notoriously difficult to compute them in all generality. Once the four-point conformal blocks are known, the CFT data are in principle constrained from associativity of the four-point

\footnotetext{
*jean-francois.fortin@phy.ulaval.ca wenjie.ma.1@ulaval.ca

witold.skiba@yale.edu
}

Published by the American Physical Society under the terms of the Creative Commons Attribution 4.0 International license. Further distribution of this work must maintain attribution to the author(s) and the published article's title, journal citation, and DOI. Funded by SCOAP ${ }^{3}$. correlation functions, the so-called conformal bootstrap introduced in [1]. For this reason, a lot of effort has been put towards computing four-point conformal blocks; see for example the Casimir equations [2], the shadow formalism [3], the weight-shifting formalism [4], integrability [5], AdS/CFT [6], and the OPE [7-12].

The study of higher-point conformal blocks is a relatively recent field of research in CFT. For example, the scalar $M$-point blocks in the comb channel in one and two spacetime dimensions as well as the scalar five-point blocks in any spacetime dimensions were first computed in $[13,14]$. In any number of dimensions, the scalar $M$-point conformal blocks in the comb channel were introduced in $[15,16]$, and the scalar six-point conformal blocks in the snowflake channel were obtained in [17]. ${ }^{1}$

The method used in $[16,17]$ to compute the higher-point conformal blocks is based on the embedding space OPE formalism introduced in $[9,10]$. Since the embedding space OPE formalism is general, it can in principle be used for any correlation functions in any topology, including internal and external quasiprimary operators in arbitrary irreducible representations of the Lorentz group. In practice, we start with known $(M-1)$-point correlation functions, express them in terms of the natural conformal cross-ratios for the OPE differential operator, trivially apply the latter

\footnotetext{
${ }^{1}$ See also [18] for specific snowflake conformal blocks in twodimensional CFTs.
} 
on the known $(M-1)$-point correlation functions, ${ }^{2}$ express the resulting $M$-point correlation functions in terms of the appropriate conformal cross-ratios, and finally evaluate the extra sums using simple hypergeometric identities. All the steps are straightforward, although the final re-summations can be somewhat tedious.

One interesting property of higher-point conformal blocks is the existence of several inequivalent topologies, as exemplified for example by the two topologies appearing at six points, namely the comb channel and the snowflake channel. In [17], we argued that the symmetry group of a given topology, denoted by $H_{M \mid \text { channel, }}$, plays an interesting role in determining the number of equivalent ways of writing the conformal blocks, or in other words the number of identities the conformal blocks must satisfy. This observation also led to an understanding of the number of $M$-point conformal bootstrap channels one should expect. Moreover, we conjectured an identity between the number of topologies and the symmetry groups, given by

$$
\sum_{1 \leq i \leq T_{0}(M)} \frac{1}{\left|H_{M \mid \text { channel } i}\right|}=\frac{T(M)}{\left|S_{M}\right|}=\frac{(2 M-5) ! !}{M !} .
$$

In (1.1), $T_{0}(M)$ is the number of inequivalent $M$-point topologies, which corresponds also to the number of unrooted binary trees with $M$ unlabeled leaves ${ }^{3} ; T(M)$ is the number of different ways of expressing the same full $M$-point correlation functions, which is given by the number of unrooted binary trees with $M$ labeled leaves; and $S_{M}$ is the symmetry group of the full $M$-point correlation functions, which is simply the symmetry group of $M$ elements.

Another interesting feature of higher-point conformal blocks is the appearance of extra sums, denoted by the $F$-function [see (2.5)], in the conformal cross-ratio power series. We argued in $[16,17]$ that the minimal number of extra sums for $M$-point conformal blocks in an arbitrary channel is $M-4$ and that it is always possible to express $F_{M}$ as $M-4$ sums (extra sums can be evaluated). This was proven by direct computation in the comb channel and for six-point conformal blocks in the snowflake channel. We also noticed that the $F$-function factorizes in the comb channel, but not in the six-point snowflake channel.

In this paper, we continue the analysis of higher-point correlation functions by computing scalar seven-point conformal blocks in all topologies, i.e., in the comb channel and the so-called extended snowflake channel. From the topologies, it is clear that the symmetry groups are

\footnotetext{
${ }^{2}$ The action of the OPE differential operator has been worked out explicitly in [10].

${ }^{3}$ Unfortunately, there does not exist an analytic expression for $T_{0}(M)$. Starting at $M=2$, the first few numbers in the sequence are $(1,1,1,1,2,2,4,6,11, \ldots)$. See The On-line Encyclopedia of Integer Sequences at https://oeis.org/A000672 and https:// oeis.org/A129860 for more details.
}

$H_{7 \mid \mathrm{comb}}=\left(\mathbb{Z}_{2}\right)^{2} \rtimes \mathbb{Z}_{2}$ and $H_{7 \mid \text { | expovenflake }}=\mathbb{Z}_{2} \times\left(\left(\mathbb{Z}_{2}\right)^{2} \rtimes \mathbb{Z}_{2}\right)$,

in agreement with (1.1). Focusing on the coset cardinalities of the two topologies $\left|S_{7} / H_{7 \mid \text { comb }}\right|=630$ and $S_{7} / H_{\left.7\right|_{\text {lnowentlake }} ^{\text {exted }}} \mid=315$, we conclude that there are 945 different ways of writing the full seven-point correlation functions.

Moreover, we prove that $F_{\left.7\right|_{\text {sxounflake }}}$ has interesting factorization properties. Although this function does not factorize into three independent sums as in the comb channel, we show that it factorizes into one sum and an object with two intertwined sums. In fact, these two factors correspond to $F_{5 \mid \text { comb }}$ and $F_{6 \mid \text { snowflake, }}$, respectively. From this observation, we conjecture rules to partially write down scalar $M$-point conformal blocks in any topology, up to the knowledge of the conformal cross-ratios.

This paper is organized as follows: Sec. II summarizes the embedding space OPE formalism. We first review the OPE differential operator in the scalar case, and then we give its action on products of conformal cross-ratios. After, we describe our notation for contributions to scalar $M$-point correlation functions from exchanged quasiprimary operators in trivial irreducible representations, and we discuss the recurrence relation transforming scalar $(M-1)$-point correlation functions to scalar $M$-point correlation functions. We finally review the scalar $M$-point conformal blocks in the comb channel as well as the scalar six-point conformal blocks in the snowflake channel before introducing the scalar seven-point conformal blocks in the extended snowflake channel. In Sec. III, we perform several consistency checks for the scalar seven-point conformal blocks in the extended snowflake channel. We start by verifying that the extended snowflake conformal blocks transform appropriately under the three generators of its topology symmetry group $H_{\left.7\right|_{\text {| }} ^{\text {extended lake }}}$. We then study the OPE limit, where two embedding space coordinates are taken to coincide, and the limit of unit operator, where one external quasiprimary operator is set to the identity operator. Finally, we conclude in Sec. IV with a lengthy discussion of the implications of our results by conjecturing rules to construct scalar conformal blocks in arbitrary topologies from partially fixed building blocks. Appendix A presents the computations necessary to obtain the scalar seven-point conformal blocks in the extended snowflake channel from the scalar six-point conformal blocks in the snowflake channel. Appendix B gives the proofs for the identities of the extended snowflake conformal blocks under the symmetry generators of $H_{\left.7\right|_{\text {snowntake }} ^{\text {extend }}}$ Lastly, Appendix C contains the remaining proofs related to the OPE limit and the limit of unit operator.

\section{HIGHER-POINT CONFORMAL BLOCKS}

This section presents the scalar seven-point conformal blocks in the extended snowflake channel. Following 
$[10,16,17]$, we first give a quick review of the OPE formalism and the scalar conformal blocks in all channels for four-, five-, and six-point correlation functions, as well as for the seven-point correlation functions in the comb channel only. We then give results for the remaining scalar seven-point conformal blocks in the extended snowflake channel, exhausting all the seven-point topologies. The relevant proofs are left for the Appendixes.

\section{A. $M$-point correlation functions from the OPE}

The form of the OPE as introduced in $[9,10]$ is applicable to operators with any spins in any dimension $d$. Using the explicit action obtained in $[8,10]$, it is straightforward to compute $M$-point correlation functions from $(M-1)$-point correlation functions. By recurrence, we can thus determine any conformal blocks by simply applying the OPE recursively in the proper order. For example, the OPE has been used to compute two-, three-, and four-point correlation functions in [11], and general rules for four-point conformal blocks in arbitrary irreducible representations, relevant for the bootstrap, were presented in [12]. Recently, higher-point scalar conformal blocks in the comb channel were obtained in [16], and scalar six-point conformal blocks in the snowflake channel were evaluated in [17] using this method.

The OPE in embedding space simplifies considerably for scalar operators because there are no free Lorentz indices,

$$
\begin{aligned}
\mathcal{O}_{i}\left(\eta_{1}\right) \mathcal{O}_{j}\left(\eta_{2}\right) & =c_{i j}^{k} \frac{1}{\left(\eta_{1} \cdot \eta_{2}\right)^{p_{i j k}}}\left(\mathcal{D}_{12}^{2}\right)^{h_{i j k}} \mathcal{O}_{k}\left(\eta_{2}\right)+\ldots, \\
p_{i j k} & =\frac{1}{2}\left(\Delta_{i}+\Delta_{j}-\Delta_{k}\right), \\
h_{i j k} & =-\frac{1}{2}\left(\Delta_{i}-\Delta_{j}+\Delta_{k}\right),
\end{aligned}
$$

where $\Delta$ 's are the dimensions of operators $\mathcal{O}(\eta)$, and $\eta_{1,2}$ are the embedding space coordinates. We omitted an infinite sum over quasiprimary operators and kept only the scalar primary in the OPE. The differential operator $\left(\mathcal{D}_{12}^{2}\right)^{h_{i j k}}$ generates the appropriate infinite towers of descendants of $\mathcal{O}_{k}\left(\eta_{2}\right)$, while $c_{i j}{ }^{k}$ is the OPE coefficient. All quantities defined in (2.1) are introduced and explained in detail in [10] as is the more complicated case of operators with spin.

The scalar differential operator $\mathcal{D}_{12}^{2}$ in (2.1) can be defined for any pairs of coordinates as

$$
\mathcal{D}_{i j}^{2}=\left(\eta_{i} \cdot \eta_{j}\right) \partial_{j}^{2}-\left(d+2 \eta_{j} \cdot \partial_{j}\right) \eta_{i} \cdot \partial_{j},
$$

where $\partial_{j}=\frac{\partial}{\partial \eta_{j}}$ and $d$ is the dimension of spacetime. $\mathcal{D}_{i j}^{2}$ is homogeneous of degree 1 with respect to $\eta_{i}$ and of degree -1 with respect to $\eta_{j}$. The scalar OPE differential operator can be made homogeneous of degree 0 with respect to all coordinates, where for brevity $\eta_{i j} \equiv \eta_{i} \cdot \eta_{j}$,

$$
\overline{\mathcal{D}}_{i j ; k l ; m}^{2}=\frac{\eta_{i j} \eta_{k l}}{\eta_{i k} \eta_{i l}} \mathcal{D}_{i j}^{2}
$$

for some $k, l$, and $m$. While $\eta_{m}$ does not appear above, it is singled out in the choice of the cross-ratios below. The action of $\overline{\mathcal{D}}_{i j ; k l ; m}^{2}$ on the conformal cross-ratios,

$$
\begin{aligned}
x_{m} & =\frac{\eta_{i j} \eta_{k l} \eta_{i m}}{\eta_{i k} \eta_{i l} \eta_{j m}}, \\
y_{a} & =1-\frac{\eta_{i m} \eta_{j a}}{\eta_{i a} \eta_{j m}}, \quad 1 \leq a \leq M, \quad a \neq i, j, m, \\
z_{a b} & =\frac{\eta_{i k} \eta_{i l} \eta_{a b}}{\eta_{k l} \eta_{i a} \eta_{i b}}, \quad 1 \leq a<b \leq M, \quad a, b \neq i, j,
\end{aligned}
$$

can be written as an infinite series of the hypergeometric type as

$$
\begin{aligned}
\overline{\mathcal{D}}_{i j ; k l ; m}^{2 h} x_{m}^{\bar{q}} \prod_{\substack{1 \leq a \leq M \\
a \neq i, j, m}}\left(1-y_{a}\right)^{-q_{a}} \\
=x_{m}^{\bar{q}+h} \sum_{\left\{n_{a}, n_{a m}, n_{a b}\right\} \geq 0} \frac{(-h)_{\bar{n}_{m}+\overline{\bar{n}}}\left(q_{m}\right)_{\bar{n}_{m}}(\bar{q}+h)_{\bar{n}-\overline{\bar{n}}}}{(\bar{q})_{\bar{n}+\bar{n}_{m}}(\bar{q}+1-d / 2)_{\bar{n}_{m}+\overline{\bar{n}}}} \\
\quad \times \prod_{\substack{1 \leq a \leq M \\
a \neq i, j, m}} \frac{\left(q_{a}\right)_{n_{a}}}{n_{a m} !\left(n_{a}-n_{a m}-\bar{n}_{a}\right) !} y_{a}^{n_{a}}\left(\frac{x_{m} z_{a m}}{y_{a}}\right)^{n_{a m}} \\
\quad \times \prod_{\substack{1 \leq a \leq b \leq M \\
a, b \neq i, j, m}} \frac{1}{n_{a b} !}\left(\frac{x_{m} z_{a b}}{y_{a} y_{b}}\right)^{n_{a b}},
\end{aligned}
$$

for any real $h$. We note here that the OPE differential operator (2.3) differs by a numerical scaling factor from the one defined in [10]. Moreover, in (2.3) we introduced $\bar{q}=$ $\sum_{\substack{1 \leq a \leq M \\ a \neq i, j}} q_{a}$ as well as

$$
\begin{aligned}
\bar{n} & =\sum_{\substack{1 \leq \leq \leq M \\
a \neq i, j, m}} n_{a}, \quad \bar{n}_{m}=\sum_{\substack{1 \leq a \leq M \\
a \neq i, j, m}} n_{a m}, \\
\bar{n}_{a} & =\sum_{\substack{1 \leq b \leq M \\
b \neq i, j, m, a}} n_{a b}, \quad \overline{\bar{n}}=\sum_{\substack{1 \leq a<b \leq M \\
a, b \neq i, j, m}} n_{a b} .
\end{aligned}
$$

On the one hand, the action of the OPE is most elementary with the choice of conformal cross-ratios shown above in (2.2). On the other hand, the expressions for conformal blocks may be simpler when written in terms of a priori unknown set of cross-ratios. Once a convenient set of cross-ratios for the conformal blocks has been found one needs to change variables between the different choices for the cross-ratios. First, it is necessary to transform an $(M-1)$-point correlation function to be given in terms of the cross-ratios that work well with the OPE differential operator. Then, one acts with with the OPE. Finally, the result is reexpressed using the cross-ratios that simplify the $M$-point conformal blocks. This last step implies that we 
explicitly evaluate series of the hypergeometric type to reach the simplest possible form for the conformal blocks.

\section{B. Scalar $M$-point correlation functions}

We now focus on the contributions from exchanged scalar quasiprimary operators with conformal dimensions $\Delta_{k_{a}}$ to $M$-point correlation functions of external scalar quasiprimary operators with conformal dimensions $\Delta_{i_{a}}$, denoted by

$$
\begin{aligned}
& \left.I_{M\left(\Delta_{k_{1}}, \ldots, \Delta_{k_{M-3}}\right)}^{\left(\Delta_{i_{2}}, \ldots, \Delta_{i_{M}}, \Delta_{i_{1}}\right)}\right|_{\text {channel }}=L_{M \mid \text { channel }}^{\left(\Delta_{\left.i_{2}, \ldots, \Delta_{i_{M}}, \Delta_{i_{1}}\right)}\right)}\left[\prod_{1 \leq a \leq M-3}\left(u_{a}^{M}\right)^{\frac{\Delta_{k_{a}}}{2}}\right] \\
& \times G_{M \mid \text { channel }}^{(d, \boldsymbol{h} ; \boldsymbol{p})}\left(\boldsymbol{u}^{M}, \boldsymbol{v}^{M}\right) .
\end{aligned}
$$

Obviously, the full scalar correlation functions are sums of $I_{M}$ for scalar exchanges as in (2.4) (the focus of this paper) and $I_{M}$ for exchanges of quasiprimary operators in nontrivial irreducible representations of the Lorentz group (not discussed here).

In (2.4), the quantity $L_{M}$ corresponds to the external operators, or the "legs," and is a product of $M$ factors of embedding space coordinates responsible for the proper scaling behavior of the correlation functions. Meanwhile, $G_{M}$ are the conformal blocks in the channel (or topology) of interest, given by

$$
\begin{aligned}
& G_{M \mid \text { channel }}^{(d, \boldsymbol{h} ; \boldsymbol{p})}\left(\boldsymbol{u}^{M}, \boldsymbol{v}^{M}\right) \\
& =\sum_{\left\{m_{a}, m_{a b}\right\} \geq 0} C_{M \mid \text { channel }}^{(d, \boldsymbol{h} ; \boldsymbol{p})}(\boldsymbol{m}, \mathbf{m}) F_{M \mid \text { channel }}^{(d, \boldsymbol{h} ; \boldsymbol{p})}(\boldsymbol{m}, \mathbf{m}) \\
& \quad \times \prod_{1 \leq a \leq M-3} \frac{\left(u_{a}^{M}\right)^{m_{a}}}{m_{a} !} \prod_{1 \leq a \leq b \leq M-3} \frac{\left(1-v_{a b}^{M}\right)^{m_{a b}}}{m_{a b} !} .
\end{aligned}
$$

Equations (2.4) and (2.5) are functions of a set of conformal cross-ratios denoted by the vector $\boldsymbol{u}^{M}$ of $u_{a}^{M}$ and the matrix $v^{M}$ of $v_{a b}^{M}$. We stress here that the best sets of conformal cross-ratios are not known a priori (there are usually more than one) and that they are dependent on the topology. The scalar conformal blocks (2.5) are thus expressed as series of the hypergeometric type in powers of the conformal crossratios, with extra sums encoded in the functions $F_{M}$. Although there is freedom in the division between $C_{M}$ and $F_{M}$, we conjectured in [17] that the latter is a function of the vector $\boldsymbol{m}$ of powers of the cross-ratios $\boldsymbol{u}^{M}$ only,

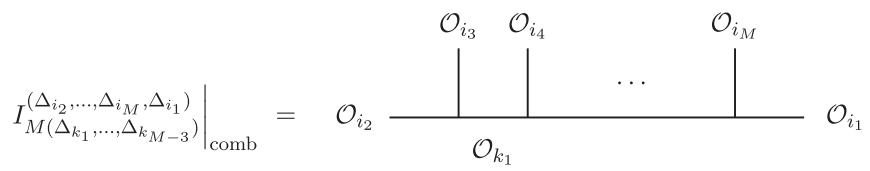

FIG. 1. Topology of scalar $M$-point conformal blocks in the comb channel.

implying it would not depend on the matrix $\mathbf{m}$ of powers of the cross-ratios $1-v^{M}$. With that in mind, we also construct $F_{M}$ such that it satisfies interesting symmetry properties related to the symmetries of the associated topology.

Finally, we mention that the vectors of parameters $\boldsymbol{h}$ and $\boldsymbol{p}$ originate from the action of the OPE (2.1). They are thus simple linear combinations of the conformal dimensions for the external and internal quasiprimary operators. This statement can be seen directly by recursion of the OPE, from which it is straightforward to obtain the following identity for some choice of $k, l$ and $m$ :

$$
\begin{aligned}
& \left.I_{M\left(\Delta_{k_{1}}, \ldots, \Delta_{k_{M-3}}\right)}^{\left(\Delta_{\left.i_{2}, \ldots, \Delta_{i_{M}}, \Delta_{i_{1}}\right)}\right)}\right|_{\substack{\text { channel } \\
M \text { points }}} \\
& \quad=\left.\frac{1}{\eta_{1 M}^{p_{M}}}\left(\frac{\eta_{k M} \eta_{l M}}{\eta_{1 M} \eta_{k l}}\right)^{h_{M-1}} \overline{\mathcal{D}}_{M 1 ; k l ; m}^{2 h_{M-1}} I_{M-1\left(\Delta_{k_{1}}, \ldots, \Delta_{k_{M-4}}\right)}^{\left(\Delta_{i_{2}}, \ldots, \Delta_{i_{M-1}}, \Delta_{k_{M-3}}\right)}\right|_{\begin{array}{c}
\text { channel } \\
M-1 \text { points }
\end{array}} \cdot
\end{aligned}
$$

It is important to point out that to reach the desired channel on the lhs of (2.6), it is necessary to start from some appropriate channels on the rhs of (2.6).

Before proceeding with the scalar seven-point conformal blocks in the extended snowflake channel, we now state the results for the scalar $M$-point correlation functions in the comb channel as well as the scalar six-point correlation functions in the snowflake channel that were obtained previously by using (2.6). While these channels were computed elsewhere, the results will be important for the factorization properties of the extended snowflake channel and for certain general observations made in Sec. IV.

\section{Scalar $M$-point correlation functions in the comb channel}

The comb channel has the topology depicted in Fig. 1. Starting from the scalar four-point correlation functions and using (2.6), we found in [16] that the $M$-point correlation functions in the comb channel can be expressed as

$$
\begin{aligned}
L_{M \mid \mathrm{comb}}^{\left(\Delta_{\left.i_{2}, \ldots, \Delta_{i_{M}}, \Delta_{i_{1}}\right)}\right.} & =\left(\frac{\eta_{34}}{\eta_{23} \eta_{24}}\right)^{\frac{\Delta_{i_{2}}}{2}}\left[\prod_{1 \leq a \leq M-2}\left(\frac{\eta_{a+1, a+3}}{\eta_{a+1, a+2} \eta_{a+2, a+3}}\right)^{\frac{\Delta_{a+2}}{2}}\right]\left(\frac{\eta_{M-1, M}}{\eta_{1, M-1} \eta_{1 M}}\right)^{\frac{\Delta_{i_{1}}}{2}}, \\
u_{a}^{M} & =\frac{\eta_{1+a, 2+a} \eta_{3+a, 4+a}}{\eta_{1+a, 3+a} \eta_{2+a, 4+a}}, \quad v_{a b}^{M}=\frac{\eta_{2-a+b, 4+b}}{\eta_{2+b, 4+b}} \prod_{1 \leq c \leq a} \frac{\eta_{3+b-c, 4+b-c}}{\eta_{2+b-c, 4+b-c}},
\end{aligned}
$$


with

$$
\begin{aligned}
C_{M \mid \mathrm{comb}}^{(d, \boldsymbol{h} ; \boldsymbol{p})}(\boldsymbol{m}, \mathbf{m})= & \frac{\left(p_{3}\right)_{m_{1}+\mathrm{tr}_{0} \mathbf{m}}\left(p_{2}+h_{2}\right)_{m_{1}+\operatorname{tr}_{1} \mathbf{m}}}{\left(p_{3}\right)_{m_{1}+\operatorname{tr}_{1} \mathbf{m}}}\left[\prod_{1 \leq a \leq M-3} \frac{\left(\bar{p}_{a+2}+\bar{h}_{a+2}\right)_{m_{a}+m_{a+1}+\bar{m}_{a}+\bar{m}_{a}}}{\left(\bar{p}_{a+2}+\bar{h}_{a+1}\right)_{2 m_{a}+\bar{m}_{a-1}+\bar{m}_{a}+\bar{m}_{a}}}\right. \\
& \left.\times\left(p_{a+2}-m_{a-1}\right)_{m_{a}+\operatorname{tr}_{a} \mathbf{m} \mathbf{m}} \frac{\left(-h_{a+2}\right)_{m_{a}}\left(-h_{a+2}+m_{a}-m_{a+1}\right)_{\bar{m}_{a-1}}}{\left(\bar{p}_{a+2}+\bar{h}_{a+1}+1-d / 2\right)_{m_{a}}}\right], \\
\operatorname{tr}_{a} \mathbf{m}= & \sum_{b} m_{b, a+b}, \quad \bar{m}_{a}=\sum_{b \leq a} m_{b a}, \quad \overline{\bar{m}}_{a}=\sum_{b>a}\left(\bar{m}_{b}-\operatorname{tr}_{b} \mathbf{m}\right),
\end{aligned}
$$

and

$$
F_{M \mid \mathrm{comb}}^{(d, \boldsymbol{h} ; \boldsymbol{p})}(\boldsymbol{m})=\prod_{1 \leq a \leq M-4}{ }_{3} F_{2}\left[\begin{array}{c}
-m_{a},-m_{a+1},-\bar{p}_{a+2}-\bar{h}_{a+1}+d / 2-m_{a} \\
p_{a+3}-m_{a}, h_{a+2}+1-m_{a}
\end{array} ;\right] .
$$

We define $\bar{p}_{a}=\sum_{b=2}^{a} p_{b}$ and $\bar{h}_{a}=\sum_{b=2}^{a} h_{b}$, while the explicit expressions for the vectors $\boldsymbol{h}$ and $\boldsymbol{p}$ in the comb channel are given by

$$
\begin{aligned}
2 h_{2} & =\Delta_{k_{1}}-\Delta_{i_{2}}-\Delta_{i_{3}}, \quad 2 h_{a}=\Delta_{k_{a-1}}-\Delta_{k_{a-2}}-\Delta_{i_{a+1}}, \\
p_{2} & =\Delta_{i_{3}}, \quad 2 p_{3}=\Delta_{i_{2}}+\Delta_{k_{1}}-\Delta_{i_{3}}, \quad 2 p_{a}=\Delta_{i_{a}}+\Delta_{k_{a-2}}-\Delta_{k_{a-3}},
\end{aligned}
$$

where $k_{M-2} \equiv i_{1}$.

As mentioned earlier, the set of conformal cross-ratios is not unique, and (2.7) was chosen based on the OPE limit while other choice are possible. Moreover, we point out that $F_{M}$ in the comb channel is a product of $M-4$ hypergeometric functions. The factorization property of $F_{M \mid \text { comb }}$ will play an important role later.

\section{Scalar six-point correlation functions in the snowflake channel}

Starting at six points, there are more topologies than the comb channel. For six-point correlation functions, the remaining topology is the snowflake channel, depicted in Fig. 2 and studied in [17].

Using the known five-point correlation functions in the comb channel and applying the OPE as in (2.6) on the appropriate quasiprimary operator, we generate the scalar six-point correlation functions in the snowflake channel for which their decomposition as in (2.4) and (2.5) is

$$
\begin{aligned}
& L_{6 \mid \text { snowflake }}^{\left(\Delta_{i_{2}}, \ldots, \Delta_{i_{6}}, \Delta_{i_{1}}\right)}=\left(\frac{\eta_{13}}{\eta_{12} \eta_{23}}\right)^{\frac{\Delta_{i_{2}}}{2}}\left(\frac{\eta_{12}}{\eta_{13} \eta_{23}}\right)^{\frac{\Delta_{i_{3}}}{2}}\left(\frac{\eta_{35}}{\eta_{34} \eta_{45}}\right)^{\frac{\Delta_{i_{4}}}{2}}\left(\frac{\eta_{34}}{\eta_{35} \eta_{45}}\right)^{\frac{\Delta_{i_{5}}}{2}}\left(\frac{\eta_{15}}{\eta_{16} \eta_{56}}\right)^{\frac{\Delta_{i_{6}}}{2}}\left(\frac{\eta_{56}}{\eta_{15} \eta_{16}}\right)^{\frac{\Delta_{i_{1}}}{2}}, \\
& u_{1}^{6}=\frac{\eta_{15} \eta_{23}}{\eta_{12} \eta_{35}}, \quad u_{2}^{6}=\frac{\eta_{13} \eta_{45}}{\eta_{15} \eta_{34}}, \quad u_{3}^{6}=\frac{\eta_{16} \eta_{35}}{\eta_{13} \eta_{56}}, \\
& v_{11}^{6}=\frac{\eta_{13} \eta_{25}}{\eta_{12} \eta_{35}}, \quad v_{12}^{6}=\frac{\eta_{14} \eta_{35}}{\eta_{15} \eta_{34}}, \quad v_{22}^{6}=\frac{\eta_{13} \eta_{24}}{\eta_{12} \eta_{34}}, \\
& v_{13}^{6}=\frac{\eta_{15} \eta_{26}}{\eta_{12} \eta_{56}}, \quad v_{23}^{6}=\frac{\eta_{15} \eta_{36}}{\eta_{13} \eta_{56}}, \quad v_{33}^{6}=\frac{\eta_{35} \eta_{46}}{\eta_{34} \eta_{56}},
\end{aligned}
$$

with

$$
\begin{aligned}
& C_{6 \mid \text { snowflake }}^{(d, \boldsymbol{m} \boldsymbol{p})}(\boldsymbol{m}, \mathbf{m})=\frac{\left(p_{2}+h_{3}\right)_{m_{1}+m_{23}}\left(p_{3}\right)_{-m_{1}+m_{2}+m_{3}+m_{12}+m_{33}}\left(-h_{3}\right)_{m_{1}+m_{11}+m_{22}+m_{13}}}{\left(p_{2}\right)_{2 m_{1}+m_{11}+m_{13}+m_{22}+m_{23}}\left(p_{2}+1-d / 2\right)_{m_{1}}} \\
& \times \frac{\left(p_{3}-h_{2}+h_{4}\right)_{m_{2}+m_{11}}\left(p_{2}+h_{2}\right)_{m_{1}-m_{2}+m_{3}+m_{13}+m_{23}}\left(-h_{4}\right)_{m_{2}+m_{12}+m_{22}+m_{33}}}{\left(p_{3}-h_{2}\right)_{2 m_{2}+m_{11}+m_{12}+m_{22}+m_{33}}\left(p_{3}-h_{2}+1-d / 2\right)_{m_{2}}} \\
& \times \frac{\left(\bar{p}_{3}+h_{2}+h_{5}\right)_{m_{3}+m_{12}}\left(-h_{2}\right)_{m_{1}+m_{2}-m_{3}+m_{11}+m_{22}}\left(-h_{5}\right)_{m_{3}+m_{13}+m_{23}+m_{33}}}{\left(\bar{p}_{3}+h_{2}\right)_{2 m_{3}+m_{12}+m_{13}+m_{23}+m_{33}}\left(\bar{p}_{3}+h_{2}+1-d / 2\right)_{m_{3}}},
\end{aligned}
$$


and

$$
\begin{aligned}
F_{6 \mid \text { snowflake }}^{(d, \boldsymbol{h} ; \boldsymbol{p})}(\boldsymbol{m})= & \frac{1}{\left(p_{3}\right)_{-m_{1}}\left(-p_{3}+h_{2}+d / 2\right)_{-m_{2}}\left(-h_{2}\right)_{-m_{3}}} F_{2,1,0}^{1,3,2}\left[\begin{array}{c}
\bar{p}_{3}-d / 2 ;-m_{2},-h_{2}, p_{3} ;-m_{1},-m_{3} \\
-h_{2}-m_{3}, p_{3}-m_{1} ; p_{3}-h_{2}+1-d / 2 ;-
\end{array} \mid 1,1\right] \\
= & \frac{1}{\left(p_{3}\right)_{-m_{1}}\left(-p_{3}+h_{2}+d / 2\right)_{-m_{2}}\left(-\bar{p}_{3}-h_{2}+d / 2\right)_{-m_{3}}} \\
& \times F_{1,1,1}^{2,1,1}\left[\begin{array}{c}
\bar{p}_{3}-d / 2, p_{3} ;-m_{2} ;-m_{3} \\
p_{3}-m_{1} ; p_{3}-h_{2}+1-d / 2 ; \bar{p}_{3}+h_{2}+1-d / 2
\end{array} \mid 1,1\right],
\end{aligned}
$$

where the vectors $\boldsymbol{h}$ and $\boldsymbol{p}$ are given by

$$
\begin{aligned}
2 h_{2} & =\Delta_{k_{3}}-\Delta_{k_{2}}-\Delta_{k_{1}}, \quad 2 h_{3}=\Delta_{i_{3}}-\Delta_{i_{2}}-\Delta_{k_{1}}, \\
2 h_{4} & =\Delta_{i_{5}}-\Delta_{i_{4}}-\Delta_{k_{2}}, \quad 2 h_{5}=\Delta_{i_{1}}-\Delta_{i_{6}}-\Delta_{k_{3}}, \\
p_{2} & =\Delta_{k_{1}}, \quad 2 p_{3}=\Delta_{k_{2}}+\Delta_{k_{3}}-\Delta_{k_{1}}, \quad 2 p_{4}=\Delta_{i_{2}}+\Delta_{i_{3}}-\Delta_{k_{1}}, \\
2 p_{5} & =\Delta_{i_{4}}+\Delta_{i_{5}}-\Delta_{k_{2}}, \quad 2 p_{6}=\Delta_{i_{6}}+\Delta_{i_{1}}-\Delta_{k_{3}} .
\end{aligned}
$$

Contrary to the comb channel where the extra sums in $F_{M \mid \text { comb }}$ factorize, the two extra sums appearing in $F_{6 \mid \text { snowflake }}$ do not factorize. In fact, (2.13) is written in terms of Kampé de Fériet functions [19], which are defined as

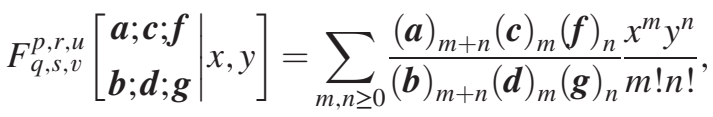

for

$$
\begin{aligned}
(\boldsymbol{a})_{m+n} & =\left(a_{1}\right)_{m+n} \cdots\left(a_{p}\right)_{m+n}, \\
(\boldsymbol{b})_{m+n} & =\left(b_{1}\right)_{m+n} \cdots\left(b_{q}\right)_{m+n}, \\
(\boldsymbol{c})_{m} & =\left(c_{1}\right)_{m} \cdots\left(c_{r}\right)_{m}, \quad(\boldsymbol{d})_{m}=\left(d_{1}\right)_{m} \cdots\left(d_{s}\right)_{m}, \\
(\boldsymbol{f})_{n} & =\left(f_{1}\right)_{n} \cdots\left(f_{u}\right)_{n}, \quad(\boldsymbol{g})_{n}=\left(g_{1}\right)_{n} \cdots\left(g_{v}\right)_{n} .
\end{aligned}
$$

We now turn to the scalar seven-point correlation functions in the extended snowflake channel.

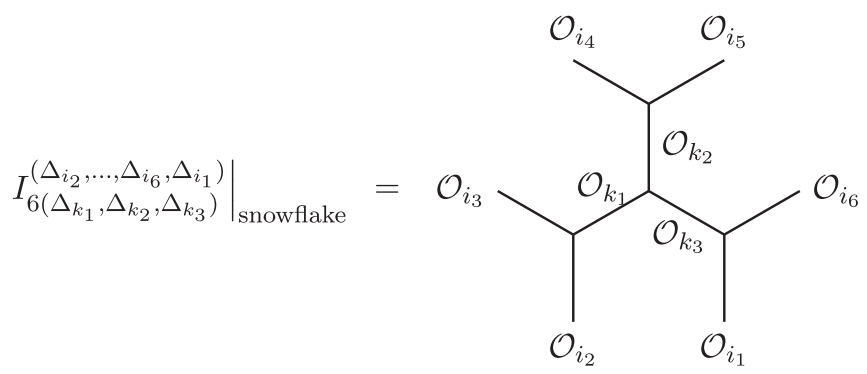

FIG. 2. Topology of the six-point conformal blocks in the snowflake channel.

\section{E. Scalar seven-point correlation functions in the extended snowflake channel}

As for six-point correlation functions, there are two different topologies for seven-point correlation functions: the comb channel discussed before and the extended snowflake channel illustrated in Fig. 3. The scalar sevenpoint correlation functions in the extended snowflake channel can be computed from the scalar six-point correlation functions in both channels.

Starting from the comb channel, it is necessary to act with the OPE on one of the two quasiprimary operators inside the comb, i.e., on $\mathcal{O}_{i_{4}}$ or $\mathcal{O}_{i_{5}}$ in Fig. 1 (acting on any one of the other quasiprimary operators leads to the seven-point comb channel). On the contrary, due to the symmetry properties of the snowflake channel seen in Fig. 2, the OPE can be applied anywhere on the snowflake to reach the extended snowflake. In Appendix A we follow the second route to compute the scalar seven-point conformal blocks in the extended snowflake channel.

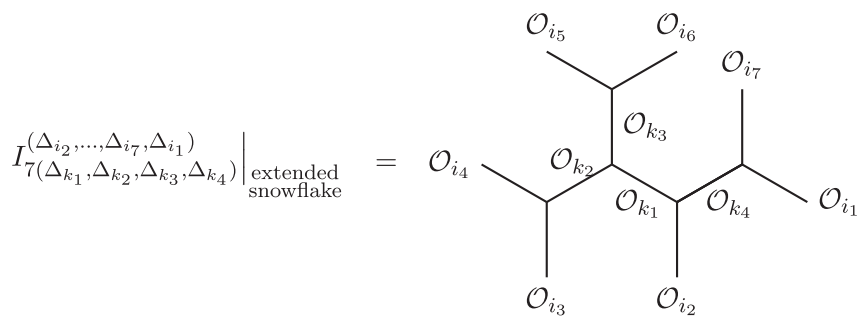

FIG. 3. Topology of the seven-point conformal blocks in the extended snowflake channel. 
To summarize, we start from the scalar six-point correlation functions in the snowflake channel (2.12), redefine the quasiprimary operators such that $\mathcal{O}_{i_{a}}\left(\eta_{a}\right) \rightarrow \mathcal{O}_{i_{a-1}}\left(\eta_{a-1}\right)$ with the knowledge that $\mathcal{O}_{i_{0}}\left(\eta_{0}\right) \equiv \mathcal{O}_{i_{6}}\left(\eta_{6}\right)$, and finally apply (2.6) with $k=5, l=6$ and $m=6$, and we obtain

$$
\begin{aligned}
& L_{\substack{7 \text { extended } \\
\text { snowilake }}}^{\left(\Delta_{i_{i}}, \ldots, \Delta_{i_{1}}, \Delta_{i_{1}}\right)}=\left(\frac{\eta_{16}}{\eta_{12} \eta_{26}}\right)^{\frac{\Delta_{i_{2}}}{2}}\left(\frac{\eta_{24}}{\eta_{23} \eta_{34}}\right)^{\frac{\Delta_{i_{3}}}{2}}\left(\frac{\eta_{23}}{\eta_{24} \eta_{34}}\right)^{\frac{\Delta_{i_{4}}}{2}}\left(\frac{\eta_{46}}{\eta_{45} \eta_{56}}\right)^{\frac{\Delta_{i_{5}}}{2}}\left(\frac{\eta_{45}}{\eta_{46} \eta_{56}}\right)^{\frac{\Delta_{i_{6}}}{2}}\left(\frac{\eta_{16}}{\eta_{17} \eta_{67}}\right)^{\frac{\Delta_{i_{7}}}{2}}\left(\frac{\eta_{67}}{\eta_{16} \eta_{17}}\right)^{\frac{\Delta_{i_{1}}}{2}}, \\
& u_{1}^{7}=\frac{\eta_{12} \eta_{46}}{\eta_{16} \eta_{24}}, \quad u_{2}^{7}=\frac{\eta_{26} \eta_{34}}{\eta_{23} \eta_{46}}, \quad u_{3}^{7}=\frac{\eta_{24} \eta_{56}}{\eta_{26} \eta_{45}}, \quad u_{4}^{7}=\frac{\eta_{17} \eta_{26}}{\eta_{12} \eta_{67}}, \\
& v_{11}^{7}=\frac{\eta_{14} \eta_{26}}{\eta_{16} \eta_{24}}, \quad v_{12}^{7}=\frac{\eta_{24} \eta_{36}}{\eta_{23} \eta_{46}}, \quad v_{13}^{7}=\frac{\eta_{15} \eta_{46}}{\eta_{16} \eta_{45}}, \quad v_{14}^{7}=\frac{\eta_{16} \eta_{27}}{\eta_{12} \eta_{67}}, \\
& v_{22}^{7}=\frac{\eta_{13} \eta_{26}}{\eta_{16} \eta_{23}}, \quad v_{23}^{7}=\frac{\eta_{25} \eta_{46}}{\eta_{26} \eta_{45}}, \quad v_{24}^{7}=\frac{\eta_{26} \eta_{37}}{\eta_{23} \eta_{67}}, \quad v_{33}^{7}=\frac{\eta_{24} \eta_{35}}{\eta_{23} \eta_{45}}, \\
& v_{34}^{7}=\frac{\eta_{26} \eta_{47}}{\eta_{24} \eta_{67}}, \quad v_{44}^{7}=\frac{\eta_{46} \eta_{57}}{\eta_{45} \eta_{67}},
\end{aligned}
$$

with

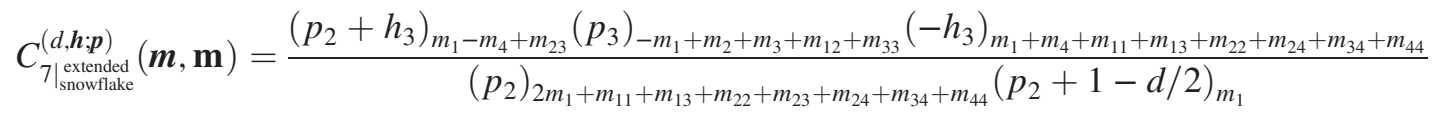

$$
\begin{aligned}
& \times \frac{\left(p_{3}-h_{2}+h_{4}\right)_{m_{2}+m_{11}+m_{34}}\left(p_{2}+h_{2}\right)_{m_{1}-m_{2}+m_{3}+m_{13}+m_{23}+m_{44}}\left(-h_{4}\right)_{m_{2}+m_{12}+m_{22}+m_{24}+m_{33}}}{\left(p_{3}-h_{2}\right)_{2 m_{2}+m_{11}+m_{12}+m_{22}+m_{24}+m_{33}+m_{34}}\left(p_{3}-h_{2}+1-d / 2\right)_{m_{2}}}
\end{aligned}
$$

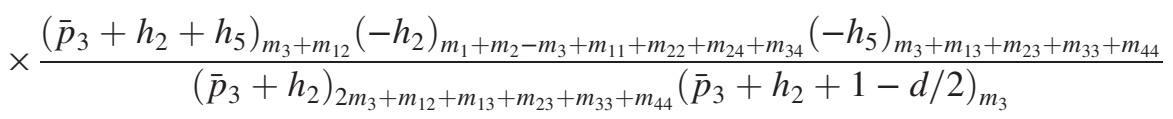

$$
\begin{aligned}
& \times \frac{\left(p_{4}-m_{1}\right)_{m_{4}+m_{14}}\left(-h_{6}\right)_{m_{4}+m_{14}+m_{24}+m_{34}+m_{44}}\left(p_{4}-h_{3}+h_{6}\right)_{m_{4}+m_{11}+m_{13}+m_{22}}}{\left(p_{2}+h_{3}+m_{1}\right)_{-m_{4}}\left(p_{4}-h_{3}\right)_{2 m_{4}+m_{11}+m_{13}+m_{14}+m_{22}+m_{24}+m_{34}+m_{44}}\left(p_{4}-h_{3}+1-d / 2\right)_{m_{4}}},
\end{aligned}
$$

as well as

$$
\begin{aligned}
& F_{\left.7\right|_{\text {snowfiake }} ^{\text {extend }}}^{(d, \boldsymbol{h} \cdot \boldsymbol{p})}(\boldsymbol{m})=\frac{1}{\left(p_{3}\right)_{-m_{1}}\left(-p_{3}+h_{2}+d / 2\right)_{-m_{2}}\left(-\bar{p}_{3}-h_{2}+d / 2\right)_{-m_{3}}}
\end{aligned}
$$

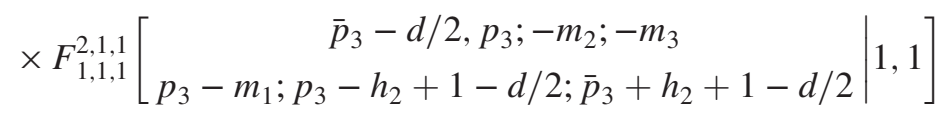

$$
\begin{aligned}
& \times_{3} F_{2}\left[\begin{array}{c}
-m_{1},-m_{4},-p_{2}+d / 2-m_{1} \\
p_{4}-m_{1}, 1-p_{2}-h_{3}-m_{1}
\end{array} ; 1\right],
\end{aligned}
$$

and finally,

$$
\begin{aligned}
& 2 h_{2}=\Delta_{k_{3}}-\Delta_{k_{2}}-\Delta_{k_{1}}, \quad 2 h_{3}=\Delta_{i_{2}}-\Delta_{k_{4}}-\Delta_{k_{1}}, \quad 2 h_{4}=\Delta_{i_{4}}-\Delta_{i_{3}}-\Delta_{k_{2}}, \\
& 2 h_{5}=\Delta_{i_{6}}-\Delta_{i_{5}}-\Delta_{k_{3}}, \quad 2 h_{6}=\Delta_{i_{1}}-\Delta_{i_{7}}-\Delta_{k_{4}}, \\
& p_{2}=\Delta_{k_{1}}, \quad 2 p_{3}=\Delta_{k_{2}}+\Delta_{k_{3}}-\Delta_{k_{1}}, \quad 2 p_{4}=\Delta_{k_{4}}+\Delta_{i_{2}}-\Delta_{k_{1}}, \\
& 2 p_{5}=\Delta_{i_{3}}+\Delta_{i_{4}}-\Delta_{k_{2}}, \quad 2 p_{6}=\Delta_{i_{5}}+\Delta_{i_{6}}-\Delta_{k_{3}}, \quad 2 p_{7}=\Delta_{i_{7}}+\Delta_{i_{1}}-\Delta_{k_{4}} .
\end{aligned}
$$

As conjectured and argued for based on consistency under several limits in [16,17], the number of extra sums for scalar seven-point conformal blocks [see (2.9) with $M=7$ and (2.18)] is three. Moreover, a comparison between (2.18) on one

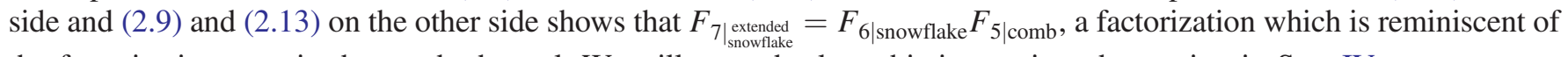
the factorization seen in the comb channel. We will come back to this interesting observation in Sec. IV.

The following sections analyze the extended snowflake results (2.16), (2.17), (2.18), and (2.19). We first verify that the symmetry properties of the extended snowflake topology extend to the scalar conformal blocks $G_{7}(2.17)$ and (2.18). 


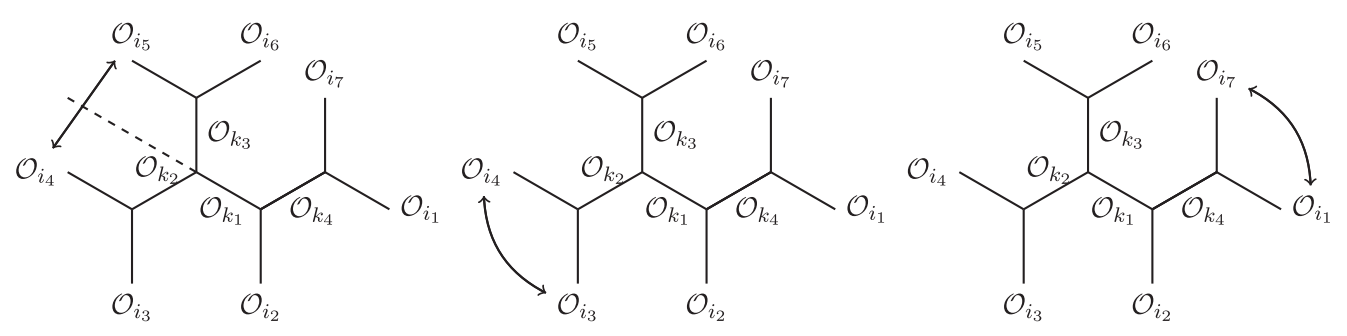

FIG. 4. Symmetries of the scalar seven-point conformal blocks in the extended snowflake channel. The figure shows the three generators with reflection (left), dendrite permutation of the first kind (middle), and dendrite permutation of the second kind (right).

We then check that the scalar conformal blocks in the extended snowflake channel exhibit the proper behavior under the OPE limit and the limit of unit operator. All proofs are left for Appendixes B and C.

\section{SANITY CHECKS}

Conformal blocks must satisfy several interesting properties. In this section, we verify that the symmetries of the scalar seven-point conformal blocks in the extended snowflake channel coincide with the symmetry group $H_{\left.7\right|_{\text {snowtlane }}}=\mathbb{Z}_{2} \times\left(\left(\mathbb{Z}_{2}\right)^{2} \rtimes \mathbb{Z}_{2}\right)$ of the extended snowflake topology. We also check that the scalar seven-point correlation functions reduce to the corresponding scalar six-point correlation functions under the OPE limit and the limit of unit operator.

\section{A. Symmetry properties}

For a given topology, there exists an associated symmetry group which we denote by $H_{M \mid \text { channel }}$. Each element of the symmetry group is a symmetry transformation of the topology, i.e., acting with a symmetry element transforms the topology back to itself. As a consequence, the scalar conformal blocks must verify several identities related to the symmetry group $H_{M \mid \text { channel }}$ where the conformal crossratios and the vectors $\boldsymbol{h}$ and $\boldsymbol{p}$ are transformed according to the symmetries. All the identities can be generated from a smaller subset of identities corresponding to the subset of generators of the symmetry group of the associated topology.
For seven-point correlation functions in the extended snowflake channel, the symmetry group is $H_{\left.7\right|_{\text {sexnendalate }}}=$ $\mathbb{Z}_{2} \times\left(\left(\mathbb{Z}_{2}\right)^{2} \rtimes \mathbb{Z}_{2}\right)$ since the extended snowflake topology is invariant under the three generators shown in Fig. 4. The first $\mathbb{Z}_{2}$ subgroup corresponds to dendrite (or OPE) permutations of the second kind, i.e., permutations $\mathcal{O}_{i_{1}} \leftrightarrow \mathcal{O}_{i_{7}}$. The second and third cyclic groups, denoted

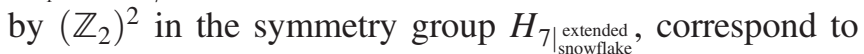
dendrite permutations of the first kind, i.e., permutations $\mathcal{O}_{i_{3}} \leftrightarrow \mathcal{O}_{i_{4}}$ as well as $\mathcal{O}_{i_{5}} \leftrightarrow \mathcal{O}_{i_{6}}$. Finally, the last $\mathbb{Z}_{2}$ subgroup corresponds to simultaneous reflections $\mathcal{O}_{i_{3}} \leftrightarrow$ $\mathcal{O}_{i_{5}}$ and $\mathcal{O}_{i_{4}} \leftrightarrow \mathcal{O}_{i_{6}}$. The latter can be seen as an internal dendrite permutation, and its effect is to switch the two external dendrites associated to the $\left(\mathbb{Z}_{2}\right)^{2}$ subgroup. This observation implies the semidirect nature of the product $\left(\mathbb{Z}_{2}\right)^{2} \rtimes \mathbb{Z}_{2}$ and distinguishes between the two different kinds (first and second) of external dendrite permutations.

Since the order of the symmetry group for the extended snowflake topology is $\left|H_{\left.7\right|_{\text {snowfiake }} \text { exted }}\right|=16$, there are sixteen equivalent ways of writing the contributions to the scalar seven-point correlation functions $I_{7}$. Hence, there are fifteen identities that the scalar seven-point conformal blocks in the extended snowflake channel $G_{7}$ must verify. We now present the three identities associated to the three generators of Fig. 4 from which all remaining identities can be obtained. The proofs are left to Appendix B.

For the reflection generator, where we choose

$$
\mathcal{O}_{i_{3}}\left(\eta_{3}\right) \leftrightarrow \mathcal{O}_{i_{5}}\left(\eta_{5}\right), \quad \mathcal{O}_{i_{4}}\left(\eta_{4}\right) \leftrightarrow \mathcal{O}_{i_{6}}\left(\eta_{6}\right), \quad \Delta_{k_{2}} \leftrightarrow \Delta_{k_{3}},
$$

the legs and conformal cross-ratios (2.16) transform as

$$
\begin{array}{rlrl}
L_{7} \prod_{1 \leq a \leq 4}\left(u_{a}^{7}\right)^{\frac{\Delta_{k}}{2}} & \rightarrow\left(v_{11}^{7}\right)^{h_{3}-h_{6}}\left(v_{12}^{7}\right)^{h_{4}}\left(v_{23}^{7}\right)^{h_{5}}\left(v_{34}^{7}\right)^{h_{6}} L_{7} \prod_{1 \leq a \leq 4}\left(u_{a}^{7}\right)^{\frac{\Delta_{a}}{2}}, \\
u_{1}^{7} & \rightarrow \frac{u_{1}^{7}}{v_{11}^{7}}, \quad u_{2}^{7} \rightarrow \frac{u_{3}^{7}}{v_{23}^{7}}, \quad u_{3}^{7} \rightarrow \frac{u_{2}^{7}}{v_{12}^{7}}, \quad u_{4}^{7} \rightarrow \frac{u_{4}^{7}}{v_{34}^{7}}, \\
v_{11}^{7} & \rightarrow \frac{1}{v_{11}^{7}}, \quad v_{12}^{7} \rightarrow \frac{1}{v_{23}^{7}}, \quad v_{13}^{7} \rightarrow \frac{v_{22}^{7}}{v_{11}^{7} v_{12}^{7}}, \quad v_{14}^{7} \rightarrow \frac{v_{11}^{7} v_{14}^{7}}{v_{34}^{7}}, \quad v_{22}^{7} \rightarrow \frac{v_{13}^{7}}{v_{11}^{7} v_{23}^{7}}, \\
v_{23}^{7} & \rightarrow \frac{1}{v_{12}^{7}}, & v_{24}^{7} & \rightarrow \frac{v_{44}^{7}}{v_{23}^{7} v_{34}^{7}}, \quad v_{33}^{7} \rightarrow \frac{v_{33}^{7}}{v_{12}^{7} v_{23}^{7}}, \quad v_{34}^{7} \rightarrow \frac{1}{v_{34}^{7}}, \quad v_{44}^{7} \rightarrow \frac{v_{24}^{7}}{v_{12}^{7} v_{34}^{7}},
\end{array}
$$


which leads to the following identity:

$$
\begin{aligned}
& G_{7 \mid \text { serended }}^{\left(d, h_{2}, h_{3}, h_{4}, h_{5}, h_{6} ; p_{2}, p_{3}, p_{4}, p_{5}, p_{6}, p_{7}\right)}\left(u_{1}^{7}, u_{2}^{7}, u_{3}^{7}, u_{4}^{7} ; v_{11}^{7}, v_{12}^{7}, v_{13}^{7}, v_{14}^{7}, v_{22}^{7}, v_{23}^{7}, v_{24}^{7}, v_{33}^{7}, v_{34}^{7}, v_{44}^{7}\right) \\
& =\left(v_{11}^{7}\right)^{h_{3}-h_{6}}\left(v_{12}^{7}\right)^{h_{4}}\left(v_{23}^{7}\right)^{h_{5}}\left(v_{34}^{7}\right)^{h_{6}} \\
& \times G_{\left.7\right|_{\text {snowferfake }}}^{\left(d,-p_{2}-h_{2}, h_{3}, h_{5}, h_{4}, h_{6} ; p_{2}, p_{3}, p_{4}, p_{6}, p_{5}, p_{7}\right)}\left(\frac{u_{1}^{7}}{v_{11}^{7}}, \frac{u_{3}^{7}}{v_{23}^{7}}, \frac{u_{2}^{7}}{v_{12}^{7}}, \frac{u_{4}^{7}}{v_{34}^{7}}, \frac{1}{v_{11}^{7}}, \frac{1}{v_{23}^{7}}, \frac{v_{22}^{7}}{v_{11}^{7} v_{12}^{7}}, \frac{v_{11}^{7} v_{14}^{7}}{v_{34}^{7}}, \frac{v_{13}^{7}}{v_{11}^{7} v_{23}^{7}}, \frac{1}{v_{12}^{7}}, \frac{v_{44}^{7}}{v_{23}^{7} v_{34}^{7}}, \frac{v_{33}^{7}}{v_{12}^{7} v_{23}^{7}}, \frac{1}{v_{34}^{7}}, \frac{v_{24}^{7}}{v_{12}^{7} v_{34}^{7}}\right) .
\end{aligned}
$$

With the help of (2.5) and using (2.17) and (2.18), (3.1) implies that $F_{7}$ does not change under the reflection generator. As such, there is an associated identity for $C_{7}$ which we prove in the Appendix B.

The generator of dendrite permutations of the first kind that we choose acts as $\mathcal{O}_{i_{3}}\left(\eta_{3}\right) \leftrightarrow \mathcal{O}_{i_{4}}\left(\eta_{4}\right)$. Hence, the legs and conformal cross-ratios (2.16) change under this generator as

$$
\begin{array}{rlrl}
L_{7} \prod_{1 \leq a \leq 4}\left(u_{a}^{7}\right)^{\frac{\Delta_{k}}{2}} & \rightarrow\left(v_{12}^{7}\right)^{-p_{3}-h_{5}}\left(v_{33}^{7}\right)^{h_{5}} L_{7} \prod_{1 \leq a \leq 4}\left(u_{a}^{7}\right)^{\frac{\Delta_{k}}{2},} \\
u_{1}^{7} & \rightarrow u_{1}^{7} v_{12}^{7}, \quad u_{2}^{7} \rightarrow \frac{u_{2}^{7}}{v_{12}^{7}}, \quad u_{3}^{7} \rightarrow \frac{u_{3}^{7}}{v_{33}^{7}}, \quad u_{4}^{7} \rightarrow u_{4}^{7}, \\
v_{11}^{7} & \rightarrow v_{22}^{7}, \quad v_{12}^{7} \rightarrow \frac{1}{v_{12}^{7}}, \quad v_{13}^{7} \rightarrow \frac{v_{12}^{7} v_{13}^{7}}{v_{33}^{7}}, \quad v_{14}^{7} \rightarrow v_{14}^{7}, \\
v_{22}^{7} & \rightarrow v_{11}^{7}, \quad v_{23}^{7} \rightarrow \frac{v_{12}^{7} v_{23}^{7}}{v_{33}^{7}}, & v_{24}^{7} \rightarrow v_{34}^{7}, \quad v_{33}^{7} \rightarrow \frac{1}{v_{33}^{7},} \\
v_{34}^{7} & \rightarrow v_{24}^{7}, & v_{44}^{7} & \rightarrow \frac{v_{12}^{7} v_{44}^{7}}{v_{33}^{7}},
\end{array}
$$

and that translates into the identity,

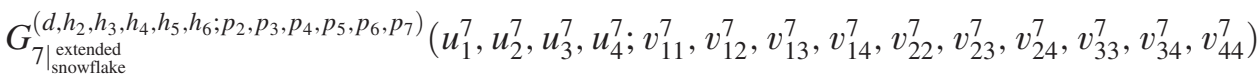

$$
\begin{aligned}
& =\left(v_{12}^{7}\right)^{-p_{3}-h_{5}}\left(v_{33}^{7}\right)^{h_{5}} \\
& \times G_{\left.7\right|_{\text {snowewlake }}}^{\left(d, h_{2}, h_{3},-p_{3}+h_{2}-h_{4}, h_{5}, h_{6} ; p_{2}, p_{3}, p_{4}, p_{5}, p_{6}, p_{7}\right)}\left(u_{1}^{7} v_{12}^{7}, \frac{u_{2}^{7}}{v_{12}^{7}}, \frac{u_{3}^{7}}{v_{33}^{7}}, u_{4}^{7} ;\right. \\
& \left.v_{22}^{7}, \frac{1}{v_{12}^{7}}, \frac{v_{12}^{7} v_{13}^{7}}{v_{33}^{7}}, v_{14}^{7}, v_{11}^{7}, \frac{v_{12}^{7} v_{23}^{7}}{v_{33}^{7}}, v_{34}^{7}, \frac{1}{v_{33}^{7}}, v_{24}^{7}, \frac{v_{12}^{7} v_{44}^{7}}{v_{33}^{7}}\right) \text {. }
\end{aligned}
$$

Again, (2.5) with (2.12) and (2.13) imply that $F_{7}$ is invariant under dendrite permutations of the first kind. Therefore, there exist a second identity for $C_{7}$.

The last generator $\mathcal{O}_{i_{1}}\left(\eta_{1}\right) \leftrightarrow \mathcal{O}_{i_{7}}\left(\eta_{7}\right)$ is the generator for dendrite permutations of the second kind, and it leads to

$$
\begin{aligned}
& L_{7} \prod_{1 \leq a \leq 4}\left(u_{a}^{7}\right)^{\frac{\Delta_{k_{a}}}{2}} \rightarrow\left(v_{14}^{7}\right)^{-p_{4}} L_{7} \prod_{1 \leq a \leq 4}\left(u_{a}^{7}\right)^{\frac{\Delta_{k_{a}}}{2}}, \\
& u_{1}^{7} \rightarrow u_{1}^{7} v_{14}^{7}, \quad u_{2}^{7} \rightarrow u_{2}^{7}, \quad u_{3}^{7} \rightarrow u_{3}^{7}, \quad u_{4}^{7} \rightarrow \frac{u_{4}^{7}}{v_{14}^{7}}, \\
& v_{11}^{7} \rightarrow v_{34}^{7}, \quad v_{12}^{7} \rightarrow v_{12}^{7}, \quad v_{13}^{7} \rightarrow v_{44}^{7}, \quad v_{14}^{7} \rightarrow \frac{1}{v_{14}^{7},} \\
& v_{22}^{7} \rightarrow v_{24}^{7}, \quad v_{23}^{7} \rightarrow v_{23}^{7}, \quad v_{24}^{7} \rightarrow v_{22}^{7}, \quad v_{33}^{7} \rightarrow v_{33}^{7}, \\
& v_{34}^{7} \rightarrow v_{11}^{7}, \quad v_{44}^{7} \rightarrow v_{13}^{7},
\end{aligned}
$$

for the legs and conformal cross-ratios (2.16). The corresponding identity for the conformal blocks is therefore 


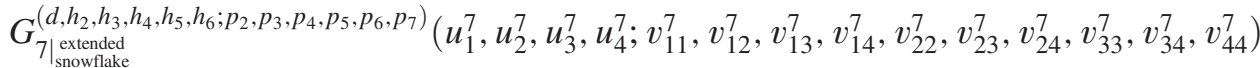

$$
\begin{aligned}
& =\left(v_{14}^{7}\right)^{-p_{4}} G_{\left.7\right|_{\text {snowflede }} ^{(\text {exted }}}^{\left(d, h_{2}, h_{3}, h_{4}, h_{5},-p_{4}+h_{3}-h_{6} ; p_{2}, p_{3}, p_{4}, p_{5}, p_{6}, p_{7}\right)}\left(u_{1}^{7} v_{14}^{7}, u_{2}^{7}, u_{3}^{7}, \frac{u_{4}^{7}}{v_{14}^{7}} ; v_{34}^{7}, v_{12}^{7}, v_{44}^{7}, \frac{1}{v_{14}^{7}}, v_{24}^{7}, v_{23}^{7}, v_{22}^{7}, v_{33}^{7}, v_{11}^{7}, v_{13}^{7}\right) \text {. }
\end{aligned}
$$

The decomposition (2.5) with (2.17) and (2.18) shows once again that $F_{7}$ given by (2.18) does not transform under this last generator, resulting in a third identity for $C_{7}$.

To summarize, the symmetry group of the extended snowflake topology is $H_{\left.7\right|_{\text {snouvtlake }} ^{\text {exted }}}=\mathbb{Z}_{2} \times\left(\left(\mathbb{Z}_{2}\right)^{2} \times \mathbb{Z}_{2}\right)$, its order is sixteen, and it is generated by the three elements shown in Fig. 4. These three generators lead to identities for the scalar seven-point conformal blocks (2.5) in the extended snowflake channel with (2.17) and (2.18). The three identities are (3.1), (3.2) and (3.3) which represent reflections, dendrite permutations of the first kind, and dendrite permutations of the second kind, respectively. It is straightforward to produce the remaining twelve equivalent representations for $I_{7}$ in the extended snowflake channel by composing the three identities above.

In the following, we investigate two limits: the OPE limit and the limit of unit operator. Due to the symmetry properties of the extended snowflake topology mentioned above, there are only two (three) limits to verify for the OPE (unit operator) case, the remaining limits being related by the action of the symmetry group.

\section{B. OPE limit}

For two external operators that emerge from the same OPE vertex in a given topology, we can define the OPE limit. In this limit, the embedding space coordinates of these two operators coincide. Therefore, the OPE limit reduces the original $M$-point correlation function to the appropriate $(M-1)$-point correlation function, up to a prefactor originating from the OPE (2.1). In the scalar case, the prefactor is obtained directly from (2.6).

For scalar seven-point correlation functions in the extended snowflake channel, as depicted in Fig. 3, there are only three possible OPE limits. They are $\eta_{3} \rightarrow \eta_{4}$, $\eta_{5} \rightarrow \eta_{6}$, and $\eta_{7} \rightarrow \eta_{1}$, respectively. With the invariance of $I_{7}$ under the symmetry group $H_{\left.7\right|_{\text {| }} ^{\text {exounfleake }} \text { end }}=\mathbb{Z}_{2} \times\left(\left(\mathbb{Z}_{2}\right)^{2} \rtimes \mathbb{Z}_{2}\right)$, we only need to investigate $I_{7}$ under two OPE limits. Here, we choose $\eta_{7} \rightarrow \eta_{1}$ (which is not related by symmetry to another pair) and $\eta_{3} \rightarrow \eta_{4}$.

For the limit $\eta_{7} \rightarrow \eta_{1}$, we have

$$
\begin{aligned}
& \left.I_{7\left(\Delta_{k_{1}}, \Delta_{k_{2}}, \Delta_{k_{3}}, \Delta_{k_{4}}\right)}^{\left(\Delta_{i_{2}}, \Delta_{i_{1}}, \Delta_{i_{1}}, \Delta_{i_{i}}, \Delta_{i}, \Delta_{i_{7}}, \Delta_{i_{1}}\right)}\right|_{\substack{\text { extended } \\
\text { snowowlake }}}
\end{aligned}
$$

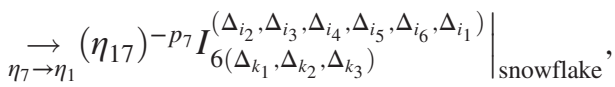

as well as

$$
\begin{array}{rlrl}
L_{7} \prod_{1 \leq a \leq 4}\left(u_{a}^{7}\right)^{\frac{\Delta_{a}}{2}} & \rightarrow\left(\eta_{17}\right)^{-p_{7}} L_{6} \prod_{1 \leq a \leq 3}\left(u_{a}^{6}\right)^{\frac{\Delta_{a}}{2}}, & \\
u_{1}^{7} & \rightarrow u_{1}^{6}, \quad u_{2}^{7} \rightarrow u_{2}^{6}, \quad u_{3}^{7} \rightarrow u_{3}^{6}, \quad u_{4}^{7} \rightarrow 0, \\
v_{11}^{7} & \rightarrow v_{11}^{6}, \quad v_{12}^{7} \rightarrow v_{12}^{6}, \quad v_{13}^{7} \rightarrow v_{13}^{6}, \quad v_{14}^{7} \rightarrow 1, \\
v_{22}^{7} & \rightarrow v_{22}^{6}, \quad v_{23}^{7} \rightarrow v_{23}^{6}, & v_{24}^{7} \rightarrow v_{22}^{6}, \quad v_{33}^{7} \rightarrow v_{33}^{6}, \\
v_{34}^{7} & \rightarrow v_{11}^{6}, \quad v_{44}^{7} \rightarrow v_{13}^{6} .
\end{array}
$$

Here, the quantities and the conformal dimensions on the rhs of the limits are the ones relevant for the six-point correlation functions in the snowflake channel, i.e., (2.11), (2.12), (2.13) and trivial substitutions for the vectors $\boldsymbol{h}$ and $\boldsymbol{p}$.

As a consequence, the OPE limit (3.4) leads to the identity $G_{7 \mid \eta_{7} \rightarrow \eta_{1}}=G_{6}$, or more precisely,

$$
\begin{aligned}
& G_{\left.7\right|_{\text {snowevflade }}}^{\left(d, h_{2}, h_{3}, h_{4}, h_{5}, h_{6} ; p_{2}, p_{3}, p_{4}, p_{5}, p_{6}, p_{7}\right)}\left(u_{1}^{6}, u_{2}^{6}, u_{3}^{6}, 0 ; v_{11}^{6}, v_{12}^{6}, v_{13}^{6}, 1, v_{22}^{6}, v_{23}^{6}, v_{22}^{6}, v_{33}^{6}, v_{11}^{6}, v_{13}^{6}\right) \\
& =G_{6 \mid \text { snowflake }}^{\left(d, h_{2}, h_{3}, h_{4}, h_{5} ; p_{2}, p_{3}, p_{4}, p_{5}, p_{6}\right)}\left(u_{1}^{6}, u_{2}^{6}, u_{3}^{6} ; v_{11}^{6}, v_{12}^{6}, v_{13}^{6}, v_{22}^{6}, v_{23}^{6}, v_{33}^{6}\right) \text {. }
\end{aligned}
$$

Since $F_{7} \rightarrow F_{6}$ in that limit, we have 


$$
\begin{aligned}
& G_{7 \mid \eta_{7} \rightarrow \eta_{1}}=\sum_{m_{a}, m_{a b} \geq 0} \frac{\left(p_{2}+h_{3}\right)_{m_{1}+m_{23}}\left(p_{3}\right)_{-m_{1}+m_{2}+m_{3}+m_{12}+m_{33}}\left(-h_{3}\right)_{m_{1}+m_{11}+m_{13}+m_{22}}}{\left(p_{2}\right)_{2 m_{1}+m_{11}+m_{13}+m_{22}+m_{23}}\left(p_{2}+1-d / 2\right)_{m_{1}}}
\end{aligned}
$$

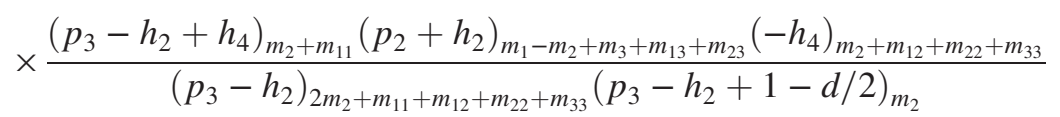

$$
\begin{aligned}
& \times \frac{\left(\bar{p}_{3}+h_{2}+h_{5}\right)_{m_{3}+m_{12}}\left(-h_{2}\right)_{m_{1}+m_{2}-m_{3}+m_{11}+m_{22}}\left(-h_{5}\right)_{m_{3}+m_{13}+m_{23}+m_{33}}}{\left(\bar{p}_{3}+h_{2}\right)_{2 m_{3}+m_{12}+m_{13}+m_{23}+m_{33}}\left(\bar{p}_{3}+h_{2}+1-d / 2\right)_{m_{3}}} \\
& \times \frac{\left(-h_{6}\right)_{m_{24}+m_{34}+m_{44}}\left(p_{4}-h_{3}+h_{6}\right)_{m_{11}+m_{13}+m_{22}-m_{24}-m_{34}-m_{44}}}{\left(p_{4}-h_{3}\right)_{m_{11}+m_{13}+m_{22}}} \\
& \times F_{6} \frac{m_{11} ! m_{13} ! m_{22} !}{\left(m_{11}-m_{34}\right) !\left(m_{13}-m_{44}\right) !\left(m_{22}-m_{24}\right) ! m_{24} ! m_{34} ! m_{44} !} \prod_{1 \leq a \leq 3} \frac{\left(u_{a}^{6}\right)^{m_{a}}}{m_{a} !} \prod_{1 \leq a \leq b \leq 3} \frac{\left(1-v_{a b}^{6}\right)^{m_{a b}}}{m_{a b} !},
\end{aligned}
$$

where we performed the following change of variables:

$$
m_{11} \rightarrow m_{11}-m_{34}, \quad m_{13} \rightarrow m_{13}-m_{44}, \quad m_{22} \rightarrow m_{22}-m_{24} .
$$

Evaluating the sums over $m_{24}, m_{34}$, and finally $m_{44}$ using standard hypergeometric resummation formula straightforwardly leads to $G_{7 \mid \eta_{7} \rightarrow \eta_{1}}=G_{6}$, proving (3.4).

Since the proof for the last independent OPE limit $\eta_{3} \rightarrow \eta_{4}$ is more intricate, it is left for Appendix C.

\section{Limit of unit operator}

The limit of unit operator consists in setting one external scalar quasiprimary operator to the identity operator. As a consequence, a given $M$-point correlation function morphs into the appropriate $(M-1)$-point correlation function.

For scalar seven-point correlation functions in the extended snowflake channel, there are only three limits of unit operator to check, thanks to the symmetry group. They are $\mathcal{O}_{i_{2}}\left(\eta_{2}\right) \rightarrow \mathbb{1}, \quad \mathcal{O}_{i_{3}}\left(\eta_{3}\right) \rightarrow \mathbb{1}$ and $\mathcal{O}_{i_{7}}\left(\eta_{7}\right) \rightarrow \mathbb{1}$, respectively.

Focusing first on the limit $\mathcal{O}_{i_{7}}\left(\eta_{7}\right) \rightarrow \mathbb{1}$, we have $\Delta_{i_{7}}=0, \Delta_{k_{4}}=\Delta_{i_{1}}$, and

$$
\begin{aligned}
& \left.I_{7\left(\Delta_{k_{1}}, \Delta_{k_{2}}, \Delta_{k_{3}}, \Delta_{k_{4}}\right)}^{\left(\Delta_{i_{2}}, \Delta_{i_{3}}, \Delta_{i}, \Delta_{i_{1}}, \Delta_{i_{6}}, \Delta_{i_{7}}, \Delta_{i_{1}}\right)}\right|_{\substack{\text { extended } \\
\text { snowflake }}} \\
& \left.\underset{\mathcal{O}_{i 7}}{\rightarrow\left(\eta_{7}\right) \rightarrow \mathbb{1}} \underset{6\left(\Delta_{k_{1}}, \Delta_{k_{2}}, \Delta_{k_{3}}\right)}{\left(\Delta_{i_{1}}, \Delta_{i_{2}}, \Delta_{i_{3}}, \Delta_{i_{4}}, \Delta_{i_{5}}, \Delta_{i_{6}}\right)}\right|_{\text {snowflake }} .
\end{aligned}
$$

In this limit, it is straightforward to see that $h_{6}=p_{7}=0$. Moreover, the remaining components of the vectors $\boldsymbol{h}$ and $\boldsymbol{p}$ (2.19) become the appropriate components of the scalar six-point correlation functions in the snowflake channel (2.14). Since the conformal cross-ratios transform as

$$
u_{a}^{7} \rightarrow u_{a}^{6} \quad(1 \leq a \leq 3), \quad v_{a b}^{7} \rightarrow v_{a b}^{6}, \quad(1 \leq a \leq b \leq 3),
$$

and the remaining conformal cross-ratios disappear due to the sums over $m_{4}, m_{14}, m_{24}, m_{34}$ and $m_{44}$ in (2.17) being trivial [thanks to the Pochhammer symbol $\left(-h_{6}\right)_{m_{4}+m_{14}+m_{24}+m_{34}+m_{44}}$ forcing $m_{4}=m_{14}=m_{24}=m_{34}=$ $\left.m_{44}=0\right]$, we obtain

$$
L_{7} \prod_{1 \leq a \leq 3}\left(u_{a}^{7}\right)^{\frac{\Delta_{k_{a}}}{2}} \rightarrow L_{6} \prod_{1 \leq a \leq 3}\left(u_{a}^{6}\right)^{\frac{\Delta_{k_{a}}}{2}}
$$

in the appropriate channels.

Furthermore, the limit of unit operator (3.5) leads to

$$
\begin{aligned}
F_{7 \mid \mathcal{O}_{i_{7}} \rightarrow \mathbb{1}}= & \frac{\left(-\bar{p}_{3}-h_{2}+d / 2-m_{3}\right)_{m_{3}}\left(-p_{3}+h_{2}+d / 2-m_{2}\right)_{m_{2}}}{\left(p_{3}\right)_{-m_{1}}} \sum_{r_{1}, r_{2} \geq 0} \frac{\left(-m_{2}\right)_{t_{1}}\left(-m_{3}\right)_{t_{2}}}{t_{1} ! t_{2} !} \\
& \times \frac{\left(p_{3}-d / 2\right)_{t_{1}+t_{2}}}{\left(\bar{p}_{3}+h_{2}+1-d / 2\right)_{t_{2}}\left(p_{3}-m_{2}\right)_{t_{1}+t_{2}}} \frac{\left(p_{3}-h_{2}+1-d / 2\right)_{t_{1}}}{\left(p_{3}\right.}
\end{aligned}
$$

which implies $F_{7} \rightarrow F_{6}$ with the help of the standard ${ }_{3} F_{2}$-hypergeometric function identity (A.3). Taking into account that $C_{7} \rightarrow C_{6}$ trivially, we thus prove that $G_{7} \rightarrow G_{6}$ in the limit of unit operator (3.5).

The two remaining limits of unit operator being longer, their proofs are left for Appendix C. 


\section{DISCUSSION AND CONCLUSION}

With the knowledge of the action of the OPE differential operator on a general product of conformal cross-ratios, the embedding space OPE formalism introduced in $[9,10]$ leads to explicit results for any conformal correlation function. Therefore, it is perfectly suited to investigate $d$-dimensional higher-point correlation functions, a nascent field of research in CFT. With the tools provided by the embedding space OPE formalism, we already determined scalar higher-point correlation functions in the comb channel in [16] and scalar six-point correlation functions in the snowflake channel in [17].

In this paper we computed the scalar seven-point conformal blocks in the extended snowflake channel, see (2.16), (2.17), (2.18), and (2.19). With that result and the scalar seven-point conformal blocks in the comb channel, the scalar conformal blocks for seven-point correlation functions are known in all topologies. We stress that all our results are derived from the OPE, as such they are exact and do not need to be verified e.g., from the Casimir equations. Nevertheless, to check the algebra, we did observe that all the symmetries [namely, the $\mathbb{Z}_{2} \times\left(\left(\mathbb{Z}_{2}\right)^{2} \rtimes \mathbb{Z}_{2}\right)$ symmetry group of the extended snowflake topology] and appropriate limits (namely, the OPE limit where two embedding space coordinates coincide, and the limit of unit operator where one external quasiprimary operator is set to the identity operator) are satisfied.

As we did in prior work, we defined the scalar conformal blocks in terms of coefficients of the power series in the conformal cross-ratios. These coefficients are products of two functions, the $C$-function which is a product of terms, and the $F$-function that contains multiple sums; see (2.5).

In [16], we argued that using OPE limits and limits of unit operators repetitively allows one to construct the $C$-function. It seems this argument can be extended to all channels. Indeed, by rewriting the vectors $\boldsymbol{h}$ and $\boldsymbol{p}$ explicitly in terms of the conformal dimensions, it is clear that a pattern emerges. Focusing on the scalar $M$-point $C$-functions in the comb channel (2.8) and the scalar sixand seven-point $C$-functions in the snowflake and extended snowflake channels (2.12) and (2.17), we have

$$
\begin{aligned}
& C_{M \mid \text { comb }}^{(d, \boldsymbol{h} ; \boldsymbol{p})}(\boldsymbol{m}, \mathbf{m})=\frac{\left(\frac{\Delta_{k_{1}}-\Delta_{i_{2}}+\Delta_{i_{3}}}{2}\right)_{m_{1}+\operatorname{tr}_{1} \mathbf{m}}\left(\frac{\Delta_{k_{1}}+\Delta_{i_{2}}-\Delta_{i_{3}}}{2}\right)_{m_{1}+\operatorname{tr}_{0} \mathbf{m}}}{\left(\Delta_{k_{1}}\right)_{2 m_{1}+\bar{m}_{1}+\overline{\bar{m}}_{1}}\left(\Delta_{k_{1}}+1-d / 2\right)_{m_{1}}} \\
& \times \frac{\left(\frac{\Delta_{k_{M-3}}-\Delta_{i_{1}}+\Delta_{i_{M}}}{2}\right)_{m_{M-3}+\bar{m}_{M-4}}\left(\frac{\Delta_{k_{M-3}}+\Delta_{i_{1}}-\Delta_{i_{M}}}{2}\right)_{m_{M-3}+\bar{m}_{M-3}+\overline{\bar{m}}_{M-3}}}{\left(\Delta_{k_{M-3}}\right)_{2 m_{M-3}+\bar{m}_{M-4}+\bar{m}_{M-3}+\overline{\bar{m}}_{M-3}}\left(\Delta_{k_{M-3}}+1-d / 2\right)_{m_{M-3}}} \\
& \times \frac{\left(\frac{\Delta_{k_{2}}-\Delta_{k_{1}}+\Delta_{i_{4}}}{2}\right)_{m_{2}-m_{1}+\operatorname{tr}_{2} \mathbf{m}}\left(\frac{\Delta_{k_{2}}+\Delta_{k_{1}}-\Delta_{i_{4}}}{2}\right)_{m_{2}+m_{1}+\bar{m}_{1}+\overline{\bar{m}}_{1}}}{\left(\Delta_{k_{2}}\right)_{2 m_{2}+\bar{m}_{1}+\bar{m}_{2}+\overline{\bar{m}}_{2}}\left(\Delta_{k_{2}}+1-d / 2\right)_{m_{2}}} \\
& \times \frac{\left(\frac{\Delta_{i_{4}}-\Delta_{k_{2}}+\Delta_{k_{1}}}{2}\right)_{m_{1}}\left(\frac{\Delta_{i_{4}}-\Delta_{k_{2}}+\Delta_{k_{1}}}{2}\right)_{m_{1}-m_{2}}}{\left(\frac{\Delta_{i_{4}}+\Delta_{k_{2}}-\Delta_{k_{1}}}{2}\right)_{-m_{1}}\left(\frac{\Delta_{i_{4}}-\Delta_{k_{2}}+\Delta_{k_{1}}}{2}\right)_{m_{1}-m_{2}}} \\
& \times \frac{\left(\frac{\Delta_{k_{3}}-\Delta_{k_{2}}+\Delta_{i_{5}}}{2}\right)_{m_{3}-m_{2}+\operatorname{tr}_{3} \mathbf{m}}\left(\frac{\Delta_{k_{3}}+\Delta_{k_{2}}-\Delta_{i_{5}}}{2}\right)_{m_{3}+m_{2}+\bar{m}_{2}+\overline{\bar{m}}_{2}}}{\left(\Delta_{k_{3}}\right)_{2 m_{3}+\bar{m}_{2}+\bar{m}_{3}+\overline{\bar{m}}_{3}}\left(\Delta_{k_{3}}+1-d / 2\right)_{m_{3}}} \\
& \times \frac{\left(\frac{\Delta_{i_{5}}-\Delta_{k_{3}}+\Delta_{k_{2}}}{2}\right)_{m_{2}}\left(\frac{\Delta_{i_{5}}-\Delta_{k_{3}}+\Delta_{k_{2}}}{2}\right)_{m_{2}-m_{3}+\bar{m}_{1}}}{\left(\frac{\Delta_{i_{5}}+\Delta_{k_{3}}-\Delta_{k_{2}}}{2}\right)_{-m_{2}}\left(\frac{\Delta_{i_{5}}-\Delta_{k_{3}}+\Delta_{k_{2}}}{2}\right)_{m_{2}-m_{3}}} \\
& \times \frac{\left(\frac{\Delta_{k_{M-3}-\Delta_{k_{M-4}}+\Delta_{i_{M-1}}}}{2}\right)_{m_{M-3}-m_{M-4}+\operatorname{tr}_{M-3} \mathbf{m}}\left(\frac{\Delta_{k_{M-3}}+\Delta_{k_{M-4}}-\Delta_{i_{M-1}}}{2}\right)_{m_{M-3}+m_{M-4}+\bar{m}_{M-4}+\overline{\bar{m}}_{M-4}}}{\left(\Delta_{k_{M-4}}\right)_{2 m_{M-4}+\bar{m}_{M-5}+\bar{m}_{M-4}+\overline{\bar{m}}_{M-4}}\left(\Delta_{k_{M-4}}+1-d / 2\right)_{m_{M-4}}} \\
& \times \frac{\left(\frac{\Delta_{i_{M-1}}-\Delta_{k_{M-3}}+\Delta_{k_{M-4}}}{2}\right)_{m_{M-4}}\left(\frac{\Delta_{i_{M-1}}-\Delta_{k_{M-3}}+\Delta_{k_{M-4}}}{2}\right)_{m_{M-4}-m_{M-3}+\bar{m}_{M-5}}}{\left(\frac{\Delta_{i_{M-1}}+\Delta_{k_{M-3}}-\Delta_{k_{M-4}}}{2}\right)_{-m_{M-4}}\left(\frac{\Delta_{i_{M-1}}-\Delta_{k_{M-3}}+\Delta_{k_{M-4}}}{2}\right)_{m_{M-4}-m_{M-3}}},
\end{aligned}
$$

as well as 


$$
\begin{aligned}
& C_{6 \mid \text { snowflake }}^{(d, \boldsymbol{h} ; \boldsymbol{p})}(\boldsymbol{m}, \mathbf{m})=\frac{\left(\frac{\Delta_{k_{1}}-\Delta_{i_{2}}+\Delta_{i_{3}}}{2}\right)_{m_{1}+m_{23}}\left(\frac{\Delta_{k_{1}}+\Delta_{i_{2}}-\Delta_{i_{3}}}{2}\right)_{m_{1}+m_{11}+m_{22}+m_{13}}}{\left(\Delta_{k_{1}}\right)_{2 m_{1}+m_{11}+m_{13}+m_{22}+m_{23}}\left(\Delta_{k_{1}}+1-d / 2\right)_{m_{1}}} \\
& \times \frac{\left(\frac{\Delta_{k_{2}}-\Delta_{i_{4}}+\Delta_{i_{5}}}{2}\right)_{m_{2}+m_{11}}\left(\frac{\Delta_{k_{2}}+\Delta_{i_{4}}-\Delta_{i_{5}}}{2}\right)_{m_{2}+m_{12}+m_{22}+m_{33}}}{\left(\Delta_{k_{2}}\right)_{2 m_{2}+m_{11}+m_{12}+m_{22}+m_{33}}\left(\Delta_{k_{2}}+1-d / 2\right)_{m_{2}}} \\
& \times \frac{\left(\frac{\Delta_{k_{3}}-\Delta_{i_{6}}+\Delta_{i_{1}}}{2}\right)_{m_{3}+m_{12}}\left(\frac{\Delta_{k_{3}}+\Delta_{i_{6}}-\Delta_{i_{1}}}{2}\right)_{m_{3}+m_{13}+m_{23}+m_{33}}}{\left(\Delta_{k_{3}}\right)_{2 m_{3}+m_{12}+m_{13}+m_{23}+m_{33}}\left(\Delta_{k_{3}}+1-d / 2\right)_{m_{3}}} \\
& \times\left(\frac{-\Delta_{k_{1}}+\Delta_{k_{2}}+\Delta_{k_{3}}}{2}\right)_{-m_{1}+m_{2}+m_{3}+m_{12}+m_{33}} \\
& \times\left(\frac{-\Delta_{k_{2}}+\Delta_{k_{3}}+\Delta_{k_{1}}}{2}\right)_{-m_{2}+m_{3}+m_{1}+m_{13}+m_{23}} \\
& \times\left(\frac{-\Delta_{k_{3}}+\Delta_{k_{1}}+\Delta_{k_{2}}}{2}\right)_{-m_{3}+m_{1}+m_{2}+m_{11}+m_{22}},
\end{aligned}
$$

and

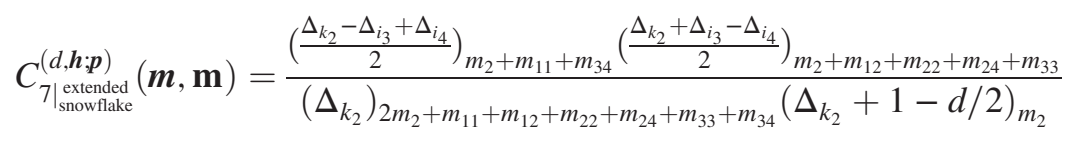

$$
\begin{aligned}
& \times \frac{\left(\frac{\Delta_{k_{3}}-\Delta_{i_{5}}+\Delta_{i_{6}}}{2}\right)_{m_{3}+m_{12}}\left(\frac{\Delta_{k_{3}}+\Delta_{i_{5}}-\Delta_{i_{6}}}{2}\right)_{m_{3}+m_{13}+m_{23}+m_{33}+m_{44}}}{\left(\Delta_{k_{3}}\right)_{2 m_{3}+m_{12}+m_{13}+m_{23}+m_{33}+m_{44}}\left(\Delta_{k_{3}}+1-d / 2\right)_{m_{3}}} \\
& \times \frac{\left(\frac{\Delta_{k_{4}}-\Delta_{i_{7}}+\Delta_{i_{1}}}{2}\right)_{m_{4}+m_{11}+m_{13}+m_{22}}\left(\frac{\Delta_{k_{4}}+\Delta_{i_{7}}-\Delta_{i_{1}}}{2}\right)_{m_{4}+m_{14}+m_{24}+m_{34}+m_{44}}}{\left(\Delta_{k_{4}}\right)_{2 m_{4}+m_{11}+m_{13}+m_{14}+m_{22}+m_{24}+m_{34}+m_{44}}\left(\Delta_{k_{4}}+1-d / 2\right)_{m_{4}}} \\
& \times \frac{\left(\frac{\Delta_{k_{1}}-\Delta_{k_{4}}+\Delta_{i_{2}}}{2}\right)_{m_{1}-m_{4}+m_{23}}\left(\frac{\Delta_{k_{1}}+\Delta_{k_{4}}-\Delta_{i_{2}}}{2}\right)_{m_{1}+m_{4}+m_{11}+m_{13}+m_{22}+m_{24}+m_{34}+m_{44}}}{\left(\Delta_{k_{1}}\right)_{2 m_{1}+m_{11}+m_{13}+m_{22}+m_{23}+m_{24}+m_{34}+m_{44}}\left(\Delta_{k_{1}}+1-d / 2\right)_{m_{1}}} \\
& \times \frac{\left(\frac{\Delta_{i_{2}}+\Delta_{k_{1}}-\Delta_{k_{4}}}{2}\right)_{m_{1}}\left(\frac{\Delta_{i_{2}}-\Delta_{k_{1}}+\Delta_{k_{4}}}{2}\right)_{-m_{1}+m_{4}+m_{14}}}{\left(\frac{\Delta_{i_{2}}-\Delta_{k_{1}}+\Delta_{k_{4}}}{2}\right)_{-m_{1}}\left(\frac{\Delta_{i_{2}}+\Delta_{k_{1}}-\Delta_{k_{4}}}{2}\right)_{m_{1}-m_{4}}} \\
& \times\left(\frac{-\Delta_{k_{1}}+\Delta_{k_{2}}+\Delta_{k_{3}}}{2}\right)_{-m_{1}+m_{2}+m_{3}+m_{12}+m_{33}} \\
& \times\left(\frac{-\Delta_{k_{2}}+\Delta_{k_{3}}+\Delta_{k_{1}}}{2}\right)_{-m_{2}+m_{3}+m_{1}+m_{13}+m_{23}+m_{44}} \\
& \times\left(\frac{-\Delta_{k_{3}}+\Delta_{k_{1}}+\Delta_{k_{2}}}{2}\right)_{-m_{3}+m_{1}+m_{2}+m_{11}+m_{22}+m_{24}+m_{34}},
\end{aligned}
$$

respectively. Hence, for a proper choice of $\boldsymbol{u}$ conformal cross-ratios behaving accordingly under the OPE limit, ${ }^{4}$ the $C$-function is given by multiplying factors of

$$
\frac{\left(\frac{\Delta_{k_{a}}-\Delta_{i}+\Delta_{j}}{2}\right)_{m_{a}+\ldots}\left(\frac{\Delta_{k_{a}}+\Delta_{i}-\Delta_{j}}{2}\right)_{m_{a}+\ldots}}{\left(\Delta_{k_{a}}\right)_{2 m_{a}+\ldots}\left(\Delta_{k_{a}}+1-d / 2\right)_{m_{a}}}
$$

for each pair of external quasiprimary operators $\mathcal{O}_{i}$ and $\mathcal{O}_{j}$ connected to an internal quasiprimary operator through the OPE,

\footnotetext{
${ }^{4}$ The $u$ conformal cross-ratios must be chosen such that only one of them vanishes for a given OPE limit.
} 


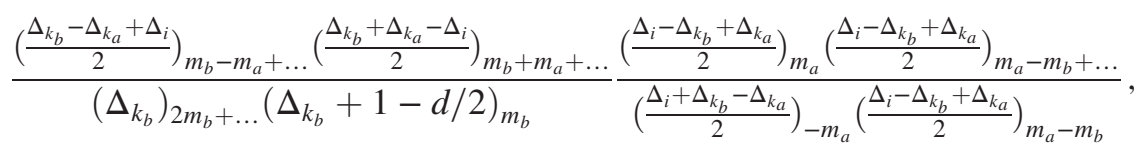

for each external quasiprimary operator $\mathcal{O}_{i}$ connected to a pair of internal quasiprimary operators $\mathcal{O}_{k_{b}}$ and $\mathcal{O}_{k_{a}}$, and

$$
\left(\frac{-\Delta_{k_{a}}+\Delta_{k_{b}}+\Delta_{k_{c}}}{2}\right)_{-m_{a}+m_{b}+m_{c}+\ldots}\left(\frac{-\Delta_{k_{b}}+\Delta_{k_{c}}+\Delta_{k_{a}}}{2}\right)_{-m_{b}+m_{c}+m_{a}+\ldots}\left(\frac{-\Delta_{k_{c}}+\Delta_{k_{a}}+\Delta_{k_{b}}}{2}\right)_{-m_{c}+m_{a}+m_{b}+\ldots},
$$

for each internal OPE with three internal quasiprimary operators $\mathcal{O}_{k_{c}}, \mathcal{O}_{k_{b}}$, and $\mathcal{O}_{k_{a}}$. Here, internal quasiprimary operators appear only once and the second factor in the rule (4.2) is not completely fixed yet (as can be seen by comparing the comb to the extended snowflake). Moreover, the dependence on the matrix of indices $\mathbf{m}$ (denoted by ellipses in the rules above) is not determined. For a proper choice of $\mathbf{v}$ conformal cross-ratios, we conjecture that the remaining ambiguities can be fixed by the OPE limit and the limit of unit operator, although it is probable that a recurring process must be used $[(M-1)$-point correlation functions must be known to verify these limits for $M$-point correlation functions].

Moreover, we observed that the $F$-functions (2.9), (2.13), and (2.18), when expressed in terms of the conformal dimensions,

$$
\begin{aligned}
F_{M \mid c o m b}^{(d, h ; p)}(\boldsymbol{m})= & { }_{3} F_{2}\left[\begin{array}{c}
-m_{1},-m_{2},-\Delta_{k_{1}}+d / 2-m_{1} \\
\frac{\Delta_{k_{2}}-\Delta_{k_{1}}+\Delta_{i_{4}}}{2}-m_{1}, \frac{\Delta_{k_{2}}-\Delta_{k_{1}}-\Delta_{i_{4}}}{2}+1-m_{1}
\end{array} ; 1\right] \\
& \times{ }_{3} F_{2}\left[\begin{array}{c}
-m_{2},-m_{3},-\Delta_{k_{2}}+d / 2-m_{2} \\
\frac{\Delta_{k_{3}}-\Delta_{k_{2}}+\Delta_{i_{5}}}{2}-m_{2}, \frac{\Delta_{k_{3}}-\Delta_{k_{2}}-\Delta_{i_{5}}}{2}+1-m_{2}
\end{array} ; 1\right] \\
& \vdots \\
& \times{ }_{3} F_{2}\left[\begin{array}{c}
-m_{M-4},-m_{M-3},-\Delta_{k_{M-4}}+d / 2-m_{M-4} \\
\frac{\Delta_{k_{M-3}}-\Delta_{k_{M-4}}+\Delta_{i_{M-1}}}{2}-m_{M-4}, \frac{\Delta_{k_{M-3}}-\Delta_{k_{M-4}}-\Delta_{i_{M-1}}}{2}+1-m_{M-4}
\end{array} ; 1\right],
\end{aligned}
$$

as well as

$$
\begin{aligned}
F_{6 \mid \text { snowflake }}^{(d, \boldsymbol{p} \boldsymbol{p})}(\boldsymbol{m})= & \frac{1}{\left(\frac{-\Delta_{k_{1}}+\Delta_{k_{2}}+\Delta_{k_{3}}}{2}\right)_{-m_{1}}\left(-\Delta_{k_{2}}+d / 2\right)_{-m_{2}}\left(-\Delta_{k_{3}}+d / 2\right)_{-m_{3}}} \\
& \times F_{1,1,1}^{2,1,1}\left[\begin{array}{c}
\frac{\Delta_{k_{1}}+\Delta_{k_{2}}+\Delta_{k_{3}}-d}{2}, \frac{-\Delta_{k_{1}}+\Delta_{k_{2}}+\Delta_{k_{3}}}{2} ;-m_{2} ;-m_{3} \\
\frac{-\Delta_{k_{1}}+\Delta_{k_{2}}+\Delta_{k_{3}}}{2}-m_{1} ; \Delta_{k_{2}}+1-d / 2 ; \Delta_{k_{3}}+1-d / 2
\end{array} \mid 1,1\right],
\end{aligned}
$$

and finally,

$$
\begin{aligned}
& F_{\left.7\right|_{\text {snowntlake }} ^{\text {extende }}}^{(\boldsymbol{m}, \boldsymbol{m})}(\boldsymbol{m})=\frac{1}{\left(\frac{-\Delta_{k_{1}}+\Delta_{k_{2}}+\Delta_{k_{3}}}{2}\right)_{-m_{1}}\left(-\Delta_{k_{2}}+d / 2\right)_{-m_{2}}\left(-\Delta_{k_{3}}+d / 2\right)_{-m_{3}}} \\
& \times F_{1,1,1}^{2,1,1}\left[\begin{array}{c|c}
\frac{\Delta_{k_{1}}+\Delta_{k_{2}}+\Delta_{k_{3}}-d}{2}, \frac{-\Delta_{k_{1}}+\Delta_{k_{2}}+\Delta_{k_{3}}}{2} ;-m_{2} ;-m_{3} \\
\frac{-\Delta_{k_{1}}+\Delta_{k_{2}}+\Delta_{k_{3}}}{2}-m_{1} ; \Delta_{k_{2}}+1-d / 2 ; \Delta_{k_{3}}+1-d / 2
\end{array} \mid 1,1\right] \\
& \times_{3} F_{2}\left[\begin{array}{c}
-m_{1},-m_{4},-\Delta_{k_{1}}+d / 2-m_{1} \\
\frac{\Delta_{k_{4}}-\Delta_{k_{1}}+\Delta_{i_{2}}}{2}-m_{1}, \frac{\Delta_{k_{4}}-\Delta_{k_{1}}-\Delta_{i_{2}}}{2}+1-m_{1}
\end{array} ; 1\right] \text {, }
\end{aligned}
$$

satisfy interesting factorization properties. Indeed, in the comb channel it was already pointed out in [16] that the $F$-function is a product of hypergeometric functions. This can be stated schematically as $F_{M \mid \text { comb }}=\left(F_{5 \mid \text { comb }}\right)^{M-4}$ with $F_{5 \mid \text { comb }}$ a building block for the $F$-function. For the snowflake channel, no such factorization occurs, implying that $F_{6 \mid \text { snowflake }}$ is another building block for the $F$-function. We now argue that $F_{5 \mid \text { comb }}$ and $F_{6 \mid \text { snowflake }}$, together with $F_{4 \mid \text { comb }}=1$, are the only 
building blocks necessary to construct the $F$-function in any topology, with the rules that the $F$-function is obtained by the multiplication of factors of

$$
{ }_{3} F_{2}\left[\begin{array}{c}
-m_{a},-m_{b},-\Delta_{k_{a}}+d / 2-m_{a} \\
\frac{\Delta_{k_{b}}-\Delta_{k_{a}}+\Delta_{i}}{2}-m_{a}, \frac{\Delta_{k_{b}}-\Delta_{k_{a}}-\Delta_{i}}{2}+1-m_{a}
\end{array} ; 1\right],
$$

for each external quasiprimary operator $\mathcal{O}_{i}$ connected to two internal quasiprimary operators $\mathcal{O}_{k_{b}}$ and $\mathcal{O}_{k_{a}}$, and factors of

$$
\frac{1}{\left(\frac{-\Delta_{k_{a}}+\Delta_{k_{b}}+\Delta_{k_{c}}}{2}\right)_{-m_{a}}\left(-\Delta_{k_{b}}+d / 2\right)_{-m_{b}}\left(-\Delta_{k_{c}}+d / 2\right)_{-m_{c}}} F_{1,1,1}^{2,1,1}\left[\begin{array}{c}
\frac{\Delta_{k_{a}}+\Delta_{k_{b}}+\Delta_{k_{c}}-d}{2}, \frac{-\Delta_{k_{a}}+\Delta_{k_{b}}+\Delta_{k_{c}}}{2} ;-m_{b} ;-m_{c} \\
\frac{-\Delta_{k_{a}}+\Delta_{k_{b}}+\Delta_{k_{c}}}{2}-m_{1} ; \Delta_{k_{b}}+1-d / 2 ; \Delta_{k_{c}}+1-d / 2
\end{array} \mid 1,1\right],
$$

for each internal OPE connecting three internal quasiprimary operators $\mathcal{O}_{k_{c}}, \mathcal{O}_{k_{b}}$ and $\mathcal{O}_{k_{a}}$.

First, we observe that this rule is respected when we consider the extended snowflake for which $F_{\left.7\right|_{\text {snowntake }}}=$ $F_{6 \mid \text { snowflake }} F_{5 \mid \text { comb }}$. The main reason why this factorization should occur for the extended snowflake again originates in the OPE limit and the limit of unit operator. It can also be understood diagrammatically directly from the topology by cutting an internal line and replacing it by a double OPE as in Fig. 5.

Indeed, concentrating on the extended snowflake, Fig. 6 leads to several identities for its $F$-function. The two identities depicted in Fig. 6 are

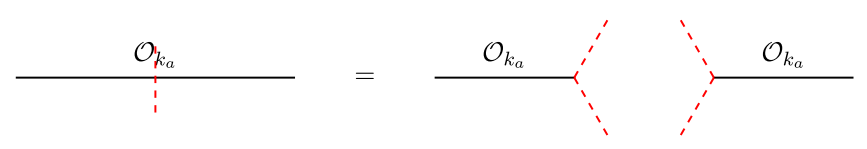

FIG. 5. Cutting procedure for the computation of the $F$ function. For a given topology, an internal line is cut and the remaining segments are both dressed as one OPE.

$$
\begin{aligned}
& F_{\left.7\right|_{\text {snowflewded }}}=F_{6 \mid \text { snowflake }} F_{5 \mid \text { comb }} \text { and } \\
& F_{\left.7\right|_{\text {snowflake }} ^{\text {extended }}}=F_{4 \mid \text { comb }} F_{\left.7\right|_{\text {snowflake }} ^{\text {extended }}},
\end{aligned}
$$

where the second identity is trivial due to $F_{4 \mid \text { comb }}=1$. Obviously, there are two extra trivial identities when the cutting procedure is performed on the internal lines with $\mathcal{O}_{k_{3}}$ and $\mathcal{O}_{k_{4}}$, respectively.

To verify that the cutting procedure of Fig. 5 makes sense, we investigate its implications for the other topologies, starting from the building blocks $F_{4 \mid \text { comb }}=1$,

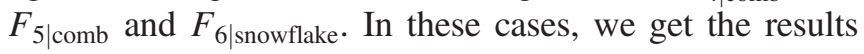
shown in Fig. 7 which imply

$$
\begin{aligned}
F_{4 \mid \mathrm{comb}} & =F_{4 \mid \mathrm{comb}} F_{4 \mid \mathrm{comb}}, \quad F_{5 \mid \mathrm{comb}}=F_{5 \mid \mathrm{comb}} F_{4 \mid \mathrm{comb}}, \\
F_{6 \mid \text { snowflake }} & =F_{6 \mid \text { snowflake }} F_{4 \mid \mathrm{comb}} .
\end{aligned}
$$

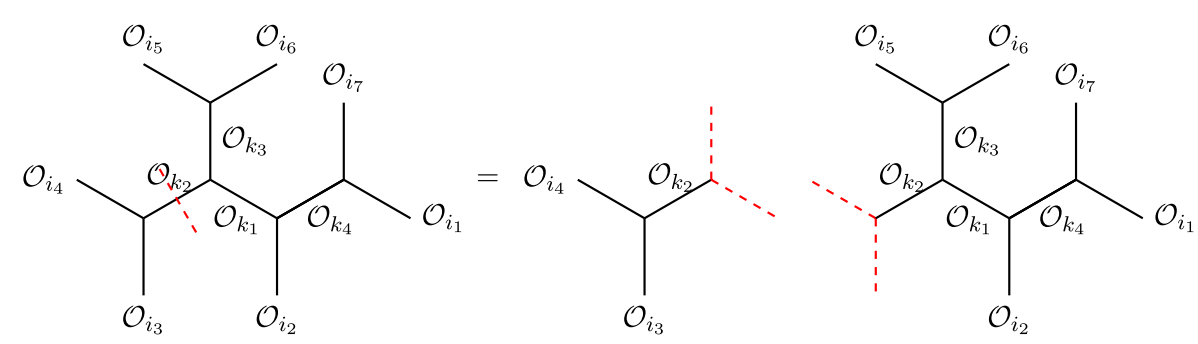

FIG. 6. Cutting procedure implemented on two different internal lines of the extended snowflake topology, leading to two factorization properties of its $F$-function. 


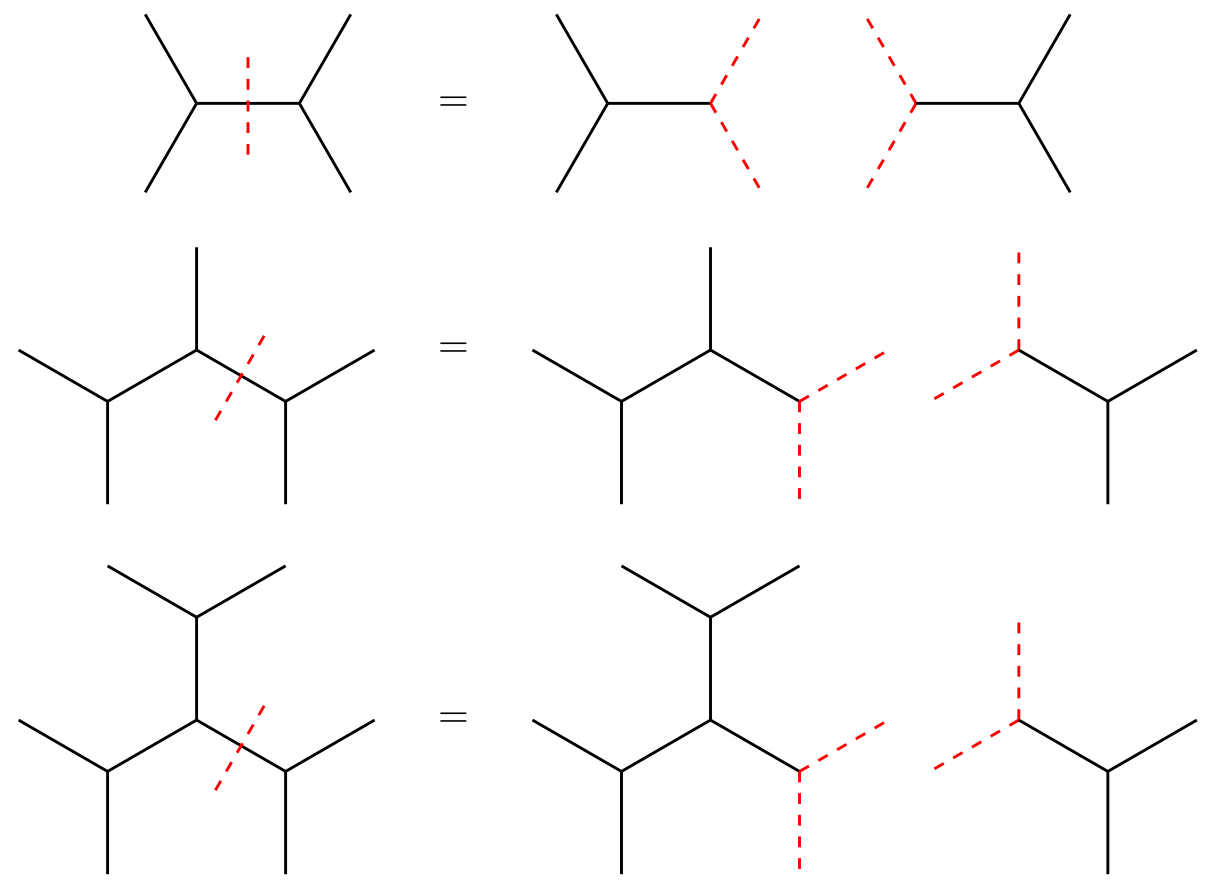

FIG. 7. Cutting procedure for the $F$-function building blocks.

These identities lead to $F_{4 \mid \text { comb }}=1$ as expected and suggest that $F_{5 \mid \text { comb }}$ and $F_{6 \mid \text { snowflake }}$ cannot be determined by the cutting procedure-they have to be computed independently, for example from the embedding space OPE formalism [16,17]. More complicated topologies always generate nontrivial factorization properties leading to the full determination of their $F$-functions from the building blocks (with the total number of extra sums appearing in $F_{M}$ always fixed to $M-4$ ).

For example, for $F_{M \mid c o m b}$, cutting the internal line with quasiprimary operator $\mathcal{O}_{k_{a}}$ in the topology depicted in Fig. 1 leads to $F_{M \mid \text { comb }}=F_{3+a \mid \text { comb }} F_{M+1-a \mid \text { comb }}$ for any $1 \leq a \leq M-3$. From its definition (2.9), this identity is verified for any $a$. Moreover, repetitively cutting the comb topology to extract factors of $F_{5 \mid \text { comb }}$ leads to the identity $F_{M \mid \mathrm{comb}}=\left(F_{5 \mid \mathrm{comb}}\right)^{M-4}$ mentioned above. Considering the ten-point correlation function in the topology of Fig. 8 as another example, we expect schematically that

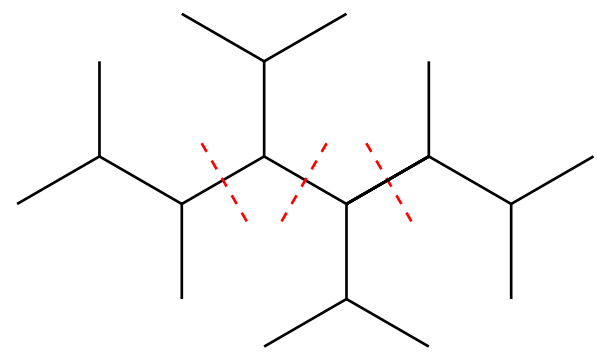

FIG. 8. Cutting procedure for one specific topology appearing in ten-point correlation functions.

$$
F_{10 \mid \text { topology of Fig. } 8}=\left(F_{5 \mid \text { comb }} F_{6 \mid \text { snowflake }}\right)^{2},
$$

where the proper parameters are determined by the quasiprimary operators as in (4.4) and (4.5).

In general, from the counting of the number of extra sums, the factorization property should imply that

$$
F_{M \mid \text { any topology }}=\left(F_{5 \mid \text { comb }}\right)^{M-4-2 n}\left(F_{6 \mid \text { snowflake }}\right)^{n},
$$

for $0 \leq n \leq\left\lfloor\frac{M-4}{2}\right\rfloor$. From Fig. 7, the factorization (4.6) should also be unique, meaning a given topology should have only one value of $n$ in the factorization that depends on the topology. Going the opposite way, one should be able to reconstruct the possible topologies by gluing the $F$-function building blocks $F_{5 \mid \text { comb }}$ and $F_{6 \mid \text { snowflake }}\left(F_{4 \mid \text { comb }}\right.$ being trivial, it can be discarded) following a gluing procedure analog to the opposite of the cutting procedure shown in Fig. 5. Indeed, for a fixed $M$-point correlation function and $n$-factorization (4.6), the gluing procedure should lead to all inequivalent topologies for fixed $M$ and $n$. Denoting the number of inequivalent $M$-point topologies with $n$-factorization as $T_{0}(M ; n)$ and taking into account the uniqueness of the factorization, this observation implies that the number of unrooted binary trees with $M$ unlabeled leaves should be expressible as

$$
T_{0}(M)=\sum_{n \geq 0}^{\left\lfloor\frac{M-4}{2}\right\rfloor} T_{0}(M ; n) .
$$

Hence, if $T_{0}(M ; n)$ can be obtained by the gluing procedure, it should lead to an expression for $T_{0}(M)$. 
Due to their symmetries, the building blocks $F_{5 \mid \text { comb }}$ and $F_{6 \mid \text { snowflake }}$ can be glued in only one independent way; however they can be glued at several different locations on an existing topology. For example, for $n=0$, the factorization (4.6) leads to $\left(F_{5 \mid \mathrm{comb}}\right)^{M-4}$ and since the $F_{5 \mid \text { comb }}$ building blocks can only be glued together in one inequivalent topology associated to the comb, we find that $T_{0}(M ; 0)=1$ corresponding to the comb channel. This is not the case when $n>0$ since the $F_{6 \mid \text { snowflake }}$ building blocks can be glued together in different topologies. Indeed, in the case of $M=8$ we have $T_{0}(8 ; 1)=2$ since the two $F_{5 \mid \text { comb }}$ building blocks can be glued in two

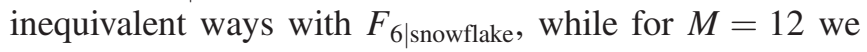
have $T_{0}(12 ; 4)=2$ because the four $F_{6 \mid \text { snowflake building }}$ blocks can be glued in two inequivalent topologies. For $n=1$, we find that $T_{0}(M ; 1)$ is given by the number of partitions of $M-6$ with at most three parts, which is given by $\left[\frac{(M-3)^{2}}{12}\right]$ where [] means rounding to the nearest integer. It would be interesting to study higher $n$-factorizations in general.

To summarize, we conjecture that there exist rules as in (4.1), (4.2) and (4.3) to construct the $C$-function as well as (4.4) and (4.5) to construct the $F$-function, for any topology. These rules rely on the OPE limit and the limit of unit operator for consistency, and they necessitate a proper set of conformal cross-ratios which is not necessarily simple to find. We hope to verify and eventually prove such rules in future work.
Finally, other research avenues worth pursuing include the conformal bootstrap from higher-point correlation functions (where the knowledge of the correlation functions with external quasiprimary operators in scalar representations only can replace the usual conformal bootstrap of four-point correlation functions when fermions are discarded), higher-point correlation functions of quasiprimary operators in arbitrary irreducible representations, and their use in the AdS/CFT correspondence (for example with respect to geodesic Witten diagrams, see for instance [14]).

\section{ACKNOWLEDGMENTS}

The authors would like to thank Sarah Hoback and Sarthak Parikh (who are completing a paper on the same topic) as well as Valentina Prilepina for useful discussions. The work of J.F. F. is supported by NSERC. W. J. M. is supported by the China Scholarship Council and in part by NSERC. The work of W. S. is supported in part by DOE HEP Grant No. DE-SC00-17660.

\section{APPENDIX A: EXTENDED SNOWFLAKE AND THE OPE}

This Appendix presents the proof leading to the scalar seven-point conformal blocks in the extended snowflake channel, starting from the OPE acting on the scalar sixpoint conformal blocks in the snowflake channel found in [17].

The proof consists in several re-summations of the hypergeometric type. For example, we use the binomial identity,

$$
(1-v)^{a+b}=\sum_{i \geq 0}(-1)^{i}\left(\begin{array}{c}
a+b \\
i
\end{array}\right) v^{i}=\sum_{i, j \geq 0}(-1)^{i+j}\left(\begin{array}{c}
a \\
i
\end{array}\right)\left(\begin{array}{c}
b \\
j
\end{array}\right) v^{i+j}
$$

as well as

$$
{ }_{2} F_{1}(a, b ; c ; z)=(1-z)^{c-a-b}{ }_{2} F_{1}(c-a, c-b ; c ; z),{ }_{2} F_{1}\left[\begin{array}{c}
-n, b \\
c
\end{array} ; 1\right]=\frac{(c-b)_{n}}{(c)_{n}},
$$

and

$$
{ }_{3} F_{2}\left[\begin{array}{c}
-n, b, c \\
d, e
\end{array} ; 1\right]=\frac{(d-b)_{n}}{(d)_{n}}{ }_{3} F_{2}\left[\begin{array}{c}
-n, b, e-c \\
b-d-n+1, e
\end{array} ; 1\right], \quad{ }_{3} F_{2}\left[\begin{array}{c}
-n, b, c \\
d, 1+b+c-d-n
\end{array} ; 1\right]=\frac{(d-b)_{n}(d-c)_{n}}{(d)_{n}(d-b-c)_{n}},
$$

for $n$ a non-negative integer.

\section{Proof of the extended snowflake}

In order to use (2.6), it is necessary to first shift the quasiprimary operators in the scalar six-point correlation functions (2.11), (2.12), and (2.13), such that $\mathcal{O}_{i_{a}}\left(\eta_{a}\right) \rightarrow \mathcal{O}_{i_{a-1}}\left(\eta_{a-1}\right)$ with $\mathcal{O}_{i_{0}}\left(\eta_{0}\right) \equiv \mathcal{O}_{i_{6}}\left(\eta_{6}\right)$. Doing so, the legs and the conformal cross-ratios transform as 


$$
\begin{array}{rlrl}
L_{6 \mid \text { snowflake }}^{\left(\Delta_{i_{2}}, \ldots, \Delta_{i_{6}}, \Delta_{k_{4}}\right)} & =\left(\frac{\eta_{16}}{\eta_{12} \eta_{26}}\right)^{\frac{\Delta_{i_{2}}}{2}}\left(\frac{\eta_{24}}{\eta_{23} \eta_{34}}\right)^{\frac{\Delta_{i 3}}{2}}\left(\frac{\eta_{23}}{\eta_{24} \eta_{34}}\right)^{\frac{\Delta_{i 4}}{2}}\left(\frac{\eta_{46}}{\eta_{45} \eta_{56}}\right)^{\frac{\Delta_{i 5}}{2}}\left(\frac{\eta_{45}}{\eta_{46} \eta_{56}}\right)^{\frac{\Delta_{i_{6}}}{2}}\left(\frac{\eta_{26}}{\eta_{12} \eta_{16}}\right)^{\frac{\Delta_{k_{4}}}{2}}, \\
u_{1}^{6} & =\frac{\eta_{12} \eta_{46}}{\eta_{16} \eta_{24}}, & u_{2}^{6}=\frac{\eta_{26} \eta_{34}}{\eta_{23} \eta_{46}}, & u_{3}^{6}=\frac{\eta_{24} \eta_{56}}{\eta_{26} \eta_{45}}, \\
v_{11}^{6} & =\frac{\eta_{14} \eta_{26}}{\eta_{16} \eta_{24}}, & v_{12}^{6}=\frac{\eta_{24} \eta_{36}}{\eta_{23} \eta_{46}}, & v_{13}^{6}=\frac{\eta_{15} \eta_{46}}{\eta_{16} \eta_{45}}, \\
v_{22}^{6} & =\frac{\eta_{13} \eta_{26}}{\eta_{16} \eta_{23}}, & v_{23}^{6}=\frac{\eta_{25} \eta_{46}}{\eta_{26} \eta_{45}}, & v_{33}^{6}=\frac{\eta_{24} \eta_{35}}{\eta_{23} \eta_{45}},
\end{array}
$$

where we also changed $\mathcal{O}_{i_{1}}\left(\eta_{1}\right) \rightarrow \mathcal{O}_{k_{4}}\left(\eta_{1}\right)$ to implement the recurrence relation (2.6). The shift also implies that the vectors $\boldsymbol{h}$ and $\boldsymbol{p}$ become the vectors (2.19) when the new components $h_{6}$ and $p_{7}$ appearing in (2.6) are included. In addition, we note that $C_{6}$ (2.12) and $F_{6}$ (2.13) are the same functions but of the new vectors $\boldsymbol{h}$ and $\boldsymbol{p}$ (2.19); thus $G_{6}$ stays the same function but of the new vectors and conformal cross-ratios (A.4).

We can now use the recurrence relation (2.6) (choosing $k=5, l=6$, and $m=6$ ) to reach the scalar seven-point conformal blocks in the extended snowflake channel. To this end, we apply the OPE differential operator following (2.3) on the conformal cross-ratios (2.2), i.e.,

$$
\begin{aligned}
x_{6}^{7} & =\frac{\eta_{17} \eta_{56}}{\eta_{57} \eta_{16}}, \quad y_{2}^{7}=1-\frac{\eta_{12} \eta_{67}}{\eta_{16} \eta_{27}}, \quad y_{3}^{7}=1-\frac{\eta_{13} \eta_{67}}{\eta_{16} \eta_{37}}, \quad y_{4}^{7}=1-\frac{\eta_{14} \eta_{67}}{\eta_{16} \eta_{47}}, \quad y_{5}^{7}=1-\frac{\eta_{15} \eta_{67}}{\eta_{16} \eta_{57}}, \\
z_{23}^{7} & =\frac{\eta_{57} \eta_{67} \eta_{23}}{\eta_{27} \eta_{37} \eta_{56}}, \quad z_{24}^{7}=\frac{\eta_{24} \eta_{57} \eta_{67}}{\eta_{27} \eta_{47} \eta_{56}}, \quad z_{25}^{7}=\frac{\eta_{25} \eta_{67}}{\eta_{27} \eta_{56}}, \quad z_{26}^{7}=\frac{\eta_{26} \eta_{57}}{\eta_{27} \eta_{56}}, \\
z_{34}^{7} & =\frac{\eta_{34} \eta_{57} \eta_{67}}{\eta_{37} \eta_{47} \eta_{56}}, \quad z_{35}^{7}=\frac{\eta_{35} \eta_{67}}{\eta_{37} \eta_{56}}, \quad z_{36}^{7}=\frac{\eta_{36} \eta_{57}}{\eta_{37} \eta_{56}}, \quad z_{45}^{7}=\frac{\eta_{45} \eta_{67}}{\eta_{47} \eta_{56}}, \quad z_{46}^{7}=\frac{\eta_{46} \eta_{57}}{\eta_{47} \eta_{56}} .
\end{aligned}
$$

Therefore, we must first reexpress the conformal cross-ratios (A.4) in terms of the conformal cross-ratios (A.5), which leads to

$$
u_{1}^{6}=\frac{1-y_{2}^{7}}{1-y_{6}^{7}} \frac{z_{46}^{7}}{z_{24}^{7}}, \quad v_{11}^{6}=\frac{1-y_{4}^{7}}{1-y_{6}^{7}} \frac{z_{26}^{7}}{z_{24}^{7}}, \quad v_{13}^{6}=\frac{1-y_{5}^{7}}{1-y_{6}^{7}} \frac{z_{46}^{7}}{z_{45}^{7}}, \quad v_{22}^{6}=\frac{1-y_{3}^{7}}{1-y_{6}^{7}} \frac{z_{26}^{7}}{z_{23}^{7}} .
$$

After acting with the OPE differential operator, we then substitute the conformal cross-ratios (A.5) with the conformal cross-ratios (2.16) using

$$
\begin{aligned}
& x_{6}^{7}=\frac{u_{1}^{7} u_{3}^{7} u_{4}^{7}}{v_{44}^{7}}, \quad y_{2}^{7}=1-\frac{1}{v_{14}^{7}}, \quad y_{3}^{7}=1-\frac{v_{22}^{7}}{v_{24}^{7}}, \quad y_{4}^{7}=1-\frac{v_{11}^{7}}{v_{34}^{7}}, \quad y_{5}^{7}=1-\frac{v_{13}^{7}}{v_{44}^{7},} \\
& z_{23}^{7}=\frac{v_{44}^{7}}{v_{14}^{7} v_{24}^{7} u_{1}^{7} u_{3}^{7}}, \quad z_{24}^{7}=\frac{v_{44}^{7}}{v_{14}^{7} v_{34}^{7} u_{1}^{7} u_{3}^{7}}, \quad z_{25}^{7}=\frac{v_{23}^{7}}{v_{14}^{7} u_{1}^{7} u_{3}^{7}}, \quad z_{26}^{7}=\frac{v_{44}^{7}}{v_{14}^{7} u_{1}^{7} u_{3}^{7}}, \\
& z_{34}^{7}=\frac{v_{44}^{7} u_{2}^{7}}{v_{24}^{7} v_{34}^{7} u_{3}^{7}}, \quad z_{35}^{7}=\frac{v_{33}^{7}}{v_{24}^{7} u_{3}^{7}}, \quad z_{36}^{7}=\frac{v_{12}^{7} v_{44}^{7}}{v_{24}^{7} u_{3}^{7}}, \quad z_{45}^{7}=\frac{1}{v_{34}^{7} u_{3}^{7}}, \quad z_{46}^{7}=\frac{v_{44}^{7}}{v_{34}^{7} u_{3}^{7}},
\end{aligned}
$$

which results in

$$
\begin{aligned}
& G_{7}=\sum(-1)^{l_{2}+l_{3}+l_{4}+l_{5}+m_{11}+m_{13}+m_{14}+m_{22}+m_{24}+m_{34}+m_{44}+k_{12}+k_{23}+k_{33}+s_{11}+s_{22}+s_{13}} \\
& \times \frac{\left(-h_{6}\right)_{m_{4}}\left(-h_{3}+n_{1}+s_{11}+s_{22}+s_{13}\right)_{\bar{r}_{6}}\left(p_{4}-h_{3}+h_{6}\right)_{m_{4}+\sum_{a=2}^{5} \sigma_{a}}}{\left(p_{4}-h_{3}\right)_{2 m_{4}+\sum_{a=2}^{5} \sigma_{a}}\left(p_{4}-h_{3}+1-d / 2\right)_{m_{4}}} \\
& \times \frac{\left(p_{4}-n_{1}\right)_{\sigma_{2}+r_{26}+\bar{r}_{2}}\left(-s_{22}\right)_{\sigma_{3}+r_{36}+\bar{r}_{3}}}{\sigma_{2} ! \sigma_{3} !} \frac{\left(-s_{11}\right)_{\sigma_{4}+r_{46}+\bar{r}_{4}}\left(-s_{13}\right)_{\sigma_{5}+r_{56}+\bar{r}_{5}}}{\sigma_{4} ! \sigma_{5} !}\left(\begin{array}{l}
n_{11} \\
s_{11}
\end{array}\right)\left(\begin{array}{l}
n_{22} \\
s_{22}
\end{array}\right)\left(\begin{array}{l}
n_{13} \\
s_{13}
\end{array}\right) \\
& \times\left(\begin{array}{c}
\sigma_{2} \\
l_{2}
\end{array}\right)\left(\begin{array}{l}
\sigma_{3} \\
l_{3}
\end{array}\right)\left(\begin{array}{l}
\sigma_{4} \\
l_{4}
\end{array}\left(\begin{array}{c}
\sigma_{5} \\
l_{5}
\end{array}\right)\left(\begin{array}{c}
l_{5} \\
m_{13}
\end{array}\right)\left(\begin{array}{c}
-p_{4}+n_{1}-r_{26}-\bar{r}_{2}-l_{2} \\
m_{14}
\end{array}\right)\left(\begin{array}{c}
l_{4} \\
m_{11}
\end{array}\right)\left(\begin{array}{l}
r_{36} \\
k_{12}
\end{array}\right)\right. \\
& \times\left(\begin{array}{c}
l_{3} \\
m_{22}
\end{array}\right)\left(\begin{array}{l}
r_{25} \\
k_{23}
\end{array}\right)\left(\begin{array}{l}
r_{35} \\
k_{33}
\end{array}\right)\left(\begin{array}{c}
s_{22}-r_{36}-\bar{r}_{3}-l_{3} \\
m_{24}
\end{array}\right)\left(\begin{array}{c}
s_{11}-r_{46}-\bar{r}_{4}-l_{4} \\
m_{34}
\end{array}\right)\left(\begin{array}{c}
s_{13}-r_{56}-\bar{r}_{5}-l_{5} \\
m_{44}
\end{array}\right) \\
& \times \frac{\left(u_{1}^{7}\right)^{n_{1}+\sum_{a, b \neq 2} r_{a b}}\left(u_{2}^{7}\right)^{n_{2}+r_{34}}\left(u_{3}^{7}\right)^{n_{3}+r_{56}}\left(u_{4}^{7}\right)^{\bar{r}_{6}+\overline{\bar{r}}} \prod_{1 \leq a \leq b \leq 4}\left(1-v_{a b}^{7}\right)^{m_{a b}}}{r_{23} ! r_{24} ! r_{25} ! r_{26} ! r_{34} ! r_{35} ! r_{36} ! r_{45} ! r_{46} ! r_{56} ! n_{1} ! n_{2} ! n_{3} ! n_{11} ! m_{12} ! n_{13} ! n_{22} ! m_{23} ! n_{33} !} C_{6} F_{6},
\end{aligned}
$$


with the appropriate legs (2.16) and also $n_{12}=m_{12}, n_{23}=m_{23}, m_{12}=n_{12}+k_{12}, m_{23}=n_{23}+k_{23}$, and $n_{33}+k_{33}=m_{33}$ after some simple manipulations (here the new indices of summation originating from the OPE are denoted by $r$ ). In (A.6) and most of the Appendixes, we omit the indices under the summation sign to avoid cluttering the equations. The scalar seven-point conformal blocks in the extended snowflake channel are thus given explicitly by (A.6), and the rest of this Appendix is dedicated to resumming the extra sums [using (A.1), (A.2) and (A.3) repetitively] to reach the results stated in (2.17) and (2.18).

We first evaluate the sums over $l_{a}$ and $\sigma_{a}$ by changing variables to

$$
\begin{array}{rlrl}
l_{3} & \rightarrow l_{3}+m_{22}, & l_{4} \rightarrow l_{4}+m_{11}, & l_{5} \rightarrow l_{5}+m_{13}, \\
\sigma_{3} \rightarrow \sigma_{3}+m_{22}, & \sigma_{4} \rightarrow \sigma_{4}+m_{11}, & \sigma_{5} \rightarrow \sigma_{5}+m_{13},
\end{array}
$$

which results in

$$
\begin{aligned}
& G_{7}=\sum(-1)^{k_{12}+k_{23}+k_{33}+s_{11}+s_{22}+s_{13}}\left(\begin{array}{c}
n_{11} \\
s_{11}
\end{array}\right)\left(\begin{array}{l}
n_{22} \\
s_{22}
\end{array}\right)\left(\begin{array}{l}
n_{13} \\
s_{13}
\end{array}\right)\left(\begin{array}{l}
r_{36} \\
k_{12}
\end{array}\right)\left(\begin{array}{l}
r_{25} \\
k_{23}
\end{array}\right)\left(\begin{array}{l}
r_{35} \\
k_{33}
\end{array}\right) \\
& \times \frac{\left(p_{4}-n_{1}\right)_{m_{14}+r_{26}+\bar{r}_{2}}\left(-s_{22}\right)_{m_{22}+m_{24}+r_{36}+\bar{r}_{3}}}{r_{23} ! r_{24} ! r_{25} ! r_{26} ! r_{34} ! r_{35} ! r_{36} ! r_{45} ! r_{46} ! r_{56} !} \frac{m_{33} !\left(-s_{11}\right)_{m_{11}+m_{34}+r_{46}+\bar{r}_{4}}\left(-s_{13}\right)_{m_{13}+m_{44}+r_{56}+\bar{r}_{5}}}{n_{11} ! n_{13} ! n_{22} ! n_{33} !} \\
& \times \frac{\left(-h_{6}\right)_{m_{4}+m_{14}+m_{24}+m_{34}+m_{44}}\left(-h_{3}+n_{1}+s_{11}+s_{22}+s_{13}\right)_{\bar{r}_{6}}\left(p_{4}-h_{3}+h_{6}\right)_{m_{4}+m_{11}+m_{13}+m_{22}}}{\left(p_{4}-h_{3}\right)_{2 m_{4}+m_{11}+m_{13}+m_{14}+m_{22}+m_{24}+m_{34}+m_{44}}\left(p_{4}-h_{3}+1-d / 2\right)_{m_{4}}} \\
& \times \frac{\left(u_{1}^{7}\right)^{n_{1}+\sum_{a, b \neq 2} r_{a b}}\left(u_{2}^{7}\right)^{n_{2}+r_{34}}\left(u_{3}^{7}\right)^{n_{3}+r_{56}}\left(u_{4}^{7}\right)^{\bar{r}_{6}+\overline{\bar{r}}}}{n_{1} ! n_{2} ! n_{3} !} \prod_{1 \leq a \leq b \leq 4} \frac{\left(1-v_{a b}^{7}\right)^{m_{a b}}}{m_{a b} !} C_{6} F_{6} .
\end{aligned}
$$

We then change variables as

$$
s_{11} \rightarrow s_{11}+m_{11}+m_{34}+r_{46}+\bar{r}_{4}, \quad s_{22} \rightarrow s_{22}+m_{22}+m_{24}+r_{36}+\bar{r}_{3}, \quad s_{13} \rightarrow s_{13}+m_{13}+m_{44}+r_{56}+\bar{r}_{5},
$$

and define a new set of summation indices by

$$
\begin{aligned}
r_{23} & =r_{23}, \quad r_{34}=r_{34}, \quad r_{46}=r_{46}, \quad r_{56}=r_{56}, \\
r_{1}^{*} & =r_{23}+r_{24}, \quad r_{2}^{*}=r_{1}^{*}+r_{25}, \quad r_{1}=r_{34}+r_{35}, \quad r_{2}=r_{1}+r_{45}, \\
r_{26} & =m_{4}-r-r_{2}^{*}, \quad r_{36}=r-r_{46}-r_{56}-r_{2},
\end{aligned}
$$

such that

$$
\begin{aligned}
& G_{7}=\sum \frac{\left(-h_{6}\right)_{m_{4}+m_{14}+m_{24}+m_{34}+m_{44}}\left(p_{4}-h_{3}+h_{6}\right)_{m_{4}+m_{11}+m_{13}+m_{22}}}{\left(p_{4}-h_{3}\right)_{2 m_{4}+m_{11}+m_{13}+m_{14}+m_{22}+m_{24}+m_{34}+m_{44}}\left(p_{4}-h_{3}+1-d / 2\right)_{m_{4}}} \\
& \times \frac{\left(p_{4}-n_{1}\right)_{m_{4}+m_{14}-r}}{r_{23} !\left(r_{1}^{*}-r_{23}\right) !\left(r_{2}^{*}-r_{1}^{*}\right) !\left(m_{4}-r-r_{2}^{*}\right) ! r_{34} !\left(r_{1}-r_{34}\right) !\left(r-r_{46}-r_{56}-r_{2}\right) !\left(r_{2}-r_{1}\right) ! r_{46} ! r_{56} !} \\
& \times \frac{m_{33} !(-1)^{k_{12}+k_{23}+k_{33}+s_{11}+s_{22}+s_{13}}}{\left(n_{13}-s_{13}-m_{13}-m_{44}-r_{56}-r_{2}-r_{2}^{*}+r_{1}^{*}+r_{34}\right) ! n_{33} !}\left(\begin{array}{c}
r-r_{46}-r_{56}-r_{2} \\
k_{12}
\end{array}\right)\left(\begin{array}{c}
r_{2}^{*}-r_{1}^{*} \\
k_{23}
\end{array}\right)\left(\begin{array}{c}
r_{1}-r_{34} \\
k_{33}
\end{array}\right) \\
& \times \frac{\left(-h_{3}+n_{1}+m_{11}+m_{13}+m_{22}+m_{24}+m_{34}+m_{44}+s_{11}+s_{22}+s_{13}+r+r_{2}+r_{2}^{*}\right)_{m_{4}-r_{2}-r_{2}^{*}}}{\left(n_{22}-s_{22}-m_{22}-m_{24}-r-r_{1}-r_{23}+r_{2}+r_{46}+r_{56}\right) !} \\
& \times \frac{1}{\left(n_{11}-s_{11}-m_{11}-m_{34}-r_{46}-r_{1}^{*}-r_{2}-r_{34}+r_{1}+r_{23}\right) ! s_{11} ! s_{13} ! s_{22} !} \\
& \times \frac{\left(u_{1}^{7}\right)^{n_{1}+r}\left(u_{2}^{7}\right)^{n_{2}+r_{34}}\left(u_{3}^{7}\right)^{n_{3}+r_{56}}\left(u_{4}^{7}\right)^{m_{4}}}{n_{1} ! n_{2} ! n_{3} !} \prod_{1 \leq a \leq b \leq 4} \frac{\left(1-v_{a b}^{7}\right)^{m_{a b}}}{m_{a b} !} C_{6} F_{6} .
\end{aligned}
$$


We can now perform the summations over $r_{23}, r_{1}^{*}, r_{2}^{*}, r_{46}, r_{1}, r_{2}$, and finally $s_{13}, 5$ leading to

$$
\begin{aligned}
& G_{7}=\sum \frac{\left(-h_{6}\right)_{m_{4}+m_{14}+m_{24}+m_{34}+m_{44}}\left(p_{4}-h_{3}+h_{6}\right)_{m_{4}+m_{11}+m_{13}+m_{22}}}{\left(p_{4}-h_{3}\right)_{2 m_{4}+m_{11}+m_{13}+m_{14}+m_{22}+m_{24}+m_{34}+m_{44}}\left(p_{4}-h_{3}+1-d / 2\right)_{m_{4}}} \\
& \times \frac{\left(-h_{3}+n_{1}+n_{11}+n_{13}+n_{22}\right)_{m_{4}-r-k_{23}}\left(p_{4}-n_{1}\right)_{m_{4}+m_{14}-r}}{\left(m_{4}-r-k_{23}\right) ! r_{34} !\left(r-r_{56}-r_{34}-k_{12}-k_{33}\right) ! r_{56} ! k_{12} ! k_{23} ! k_{33} !} \\
& \times \frac{m_{33} !(-1)^{r-r_{34}-r_{56}+k_{23}+s_{11}+s_{22}}\left(-r_{56}-k_{12}\right)_{n_{13}-m_{13}-m_{44}-r_{56}-k_{23}-k_{33}}}{\left(n_{13}-m_{13}-m_{44}-r_{56}-k_{23}-k_{33}\right) ! n_{33} !} \\
& \times \frac{\left(-h_{3}+n_{1}+n_{13}+m_{4}+m_{11}+m_{22}+m_{24}+m_{34}+s_{11}+s_{22}+r_{34}-r_{56}-k_{23}\right)_{m_{13}+m_{44}-n_{13}+r+r_{56}+k_{23}-r_{34}}}{\left(n_{22}-s_{22}-m_{22}-m_{24}-r_{34}-k_{12}-k_{33}\right) !} \\
& \times \frac{\left(-n_{11}-n_{22}+s_{11}+s_{22}+m_{11}+m_{22}+m_{24}+m_{34}+2 r_{34}+k_{12}+k_{33}\right)_{r-r_{34}-r_{56}-k_{12}-k_{33}}}{\left(n_{11}-s_{11}-m_{11}-m_{34}-r_{34}\right) ! s_{11} ! s_{22} !} \\
& \times \frac{\left(u_{1}^{7}\right)^{n_{1}+r}\left(u_{2}^{7}\right)^{n_{2}+r_{34}}\left(u_{3}^{7}\right)^{n_{3}+r_{56}}\left(u_{4}^{7}\right)^{m_{4}}}{n_{1} ! n_{2} ! n_{3} !} \prod_{1 \leq a \leq b \leq 4} \frac{\left(1-v_{a b}^{7}\right)^{m_{a b}}}{m_{a b} !} C_{6} F_{6} .
\end{aligned}
$$

After defining $s_{22}=s-s_{11}$, we evaluate the sum over $s_{11}$ and $s$, giving

$$
\begin{aligned}
& G_{7}=\sum \frac{\left(-h_{6}\right)_{m_{4}+m_{14}+m_{24}+m_{34}+m_{44}}\left(p_{4}-h_{3}+h_{6}\right)_{m_{4}+m_{11}+m_{13}+m_{22}}}{\left(p_{4}-h_{3}\right)_{2 m_{4}+m_{11}+m_{13}+m_{14}+m_{22}+m_{24}+m_{34}+m_{44}}\left(p_{4}-h_{3}+1-d / 2\right)_{m_{4}}} \\
& \times \frac{\left(-m_{13}-m_{44}+n_{13}+r_{34}-r-r_{56}-k_{23}\right)_{n_{11}+n_{22}-m_{11}-m_{22}-m_{24}-m_{34}-r-r_{34}+r_{56}}\left(p_{4}-n_{1}\right)_{m_{4}+m_{14}-r}}{\left(m_{4}-r-k_{23}\right) ! r_{34} !\left(r-r_{56}-r_{34}-k_{12}-k_{33}\right) ! r_{56} ! k_{12} ! k_{23} ! k_{33} !} \\
& \times \frac{m_{33} !(-1)^{r-r_{34}-r_{56}+k_{23}}\left(-r_{56}-k_{12}\right)_{n_{13}-m_{13}-m_{44}-r_{56}-k_{23}-k_{33}}}{\left(n_{13}-m_{13}-m_{44}-r_{56}-k_{23}-k_{33}\right) !\left(n_{22}-m_{22}-m_{24}-r_{34}-k_{12}-k_{33}\right) ! n_{33} !} \\
& \times \frac{\left(-n_{11}-n_{22}+m_{11}+m_{22}+m_{24}+m_{34}+2 r_{34}+k_{12}+k_{33}\right)_{r-r_{34}-r_{56}-k_{12}-k_{33}}}{\left(n_{11}-m_{11}-m_{34}-r_{34}\right) !} \\
& \times \frac{\left(p_{2}+h_{3}\right)_{n_{1}+n_{23}}\left(p_{3}-h_{2}+h_{4}\right)_{n_{2}+n_{11}}\left(\bar{p}_{3}+h_{2}+h_{5}\right)_{n_{3}+n_{12}}}{\left(\bar{p}_{3}+h_{2}\right)_{2 n_{3}+n_{12}+n_{13}+n_{23}+n_{33}\left(\bar{p}_{3}+h_{2}+1-d / 2\right)_{n_{3}}}} \\
& \times \frac{\left(p_{3}\right)_{n_{2}+n_{3}-n_{1}+n_{12}+n_{33}}\left(p_{2}+h_{2}\right)_{n_{1}-n_{2}+n_{3}+n_{13}+n_{23}}\left(-h_{2}\right)_{n_{1}+n_{2}-n_{3}+n_{11}+n_{22}}}{\left(p_{2}\right)_{2 n_{1}+n_{11}+n_{22}+n_{13}+n_{23}}\left(p_{2}+1-d / 2\right)_{n_{1}}} \\
& \times \frac{\left(-h_{3}\right)_{m_{1}+m_{4}+m_{11}+m_{13}+m_{22}+m_{24}+m_{34}+m_{44}}\left(-h_{4}\right)_{n_{2}+n_{12}+n_{22}+n_{33}}\left(-h_{5}\right)_{n_{3}+n_{13}+n_{23}+n_{33}}}{\left(p_{3}-h_{2}\right)_{2 n_{2}+n_{11}+n_{12}+n_{22}+n_{33}}\left(p_{3}-h_{2}+1-d / 2\right)_{n_{2}}} \\
& \times \frac{\left(u_{1}^{7}\right)^{n_{1}+r}\left(u_{2}^{7}\right)^{n_{2}+r_{34}}\left(u_{3}^{7}\right)^{n_{3}+r_{56}}\left(u_{4}^{7}\right)^{m_{4}}}{n_{1} ! n_{2} ! n_{3} !} \prod_{1 \leq a \leq b \leq 4} \frac{\left(1-v_{a b}^{7}\right)^{m_{a b}}}{m_{a b} !} F_{6} \text {, }
\end{aligned}
$$

where we explicitly expanded $C_{6}(2.12)$.

\footnotetext{
${ }^{5}$ To evaluate the sums over $r_{2}^{*}, r_{1}$ and $r_{2}$, we first change variables by

$$
r_{2}^{*} \rightarrow r_{2}^{*}+k_{23}, \quad r_{1} \rightarrow r_{1}+r_{34}+k_{33}, \quad r_{2} \rightarrow r_{2}+r_{34}+k_{33} .
$$
}


We then define $n_{22}=n-n_{11}$ and change variables by $n_{11} \rightarrow n_{11}+m_{11}+m_{34}+r_{34}$. The sum over $n_{11}$ thus leads to

$$
\begin{aligned}
& G_{7}=\sum \frac{\left(-h_{6}\right)_{m_{4}+m_{14}+m_{24}+m_{34}+m_{44}}\left(p_{4}-h_{3}+h_{6}\right)_{m_{4}+m_{11}+m_{13}+m_{22}}}{\left(p_{4}-h_{3}\right)_{2 m_{4}+m_{11}+m_{13}+m_{14}+m_{22}+m_{24}+m_{34}+m_{44}}\left(p_{4}-h_{3}+1-d / 2\right)_{m_{4}}} \\
& \times \frac{\left(-m_{13}-m_{44}+n_{13}+r_{34}-r-r_{56}-k_{23}\right)_{n-m_{11}-m_{22}-m_{24}-m_{34}-r-r_{34}+r_{56}}\left(p_{4}-n_{1}\right)_{m_{4}+m_{14}-r}}{\left(m_{4}-r-k_{23}\right) ! r_{34} !\left(r-r_{56}-r_{34}-k_{12}-k_{33}\right) ! r_{56} ! k_{12} ! k_{23} ! k_{33} !} \\
& \times \frac{m_{33} !(-1)^{r-r_{34}-r_{56}+k_{23}}\left(-r_{56}-k_{12}\right)_{n_{13}-m_{13}-m_{44}-r_{56}-k_{23}-k_{33}}}{\left(n_{13}-m_{13}-m_{44}-r_{56}-k_{23}-k_{33}\right) ! n_{33} !} \\
& \times \frac{\left(-n+m_{11}+m_{22}+m_{24}+m_{34}+2 r_{34}+k_{12}+k_{33}\right)_{r-r_{34}-r_{56}-k_{12}-k_{33}}}{\left(n-m_{11}-m_{34}-m_{22}-m_{24}-2 r_{34}-k_{12}-k_{33}\right) !} \\
& \times \frac{\left(p_{2}+h_{3}\right)_{n_{1}+n_{23}}\left(p_{3}-h_{2}+h_{4}\right)_{m_{2}+m_{11}+m_{34}}\left(\bar{p}_{3}+h_{2}+h_{5}\right)_{n_{3}+n_{12}}}{\left(\bar{p}_{3}+h_{2}\right)_{2 n_{3}+n_{12}+n_{13}+n_{23}+n_{33}}\left(\bar{p}_{3}+h_{2}+1-d / 2\right)_{n_{3}}} \\
& \times \frac{\left(p_{3}\right)_{n_{2}+n_{3}-n_{1}+n_{12}+n_{33}}\left(p_{2}+h_{2}\right)_{n_{1}-n_{2}+n_{3}+n_{13}+n_{23}}\left(-h_{2}\right)_{n_{1}+n_{2}-n_{3}+n}}{\left(p_{2}\right)_{2 n_{1}+n+n_{13}+n_{23}}\left(p_{2}+1-d / 2\right)_{n_{1}}}
\end{aligned}
$$

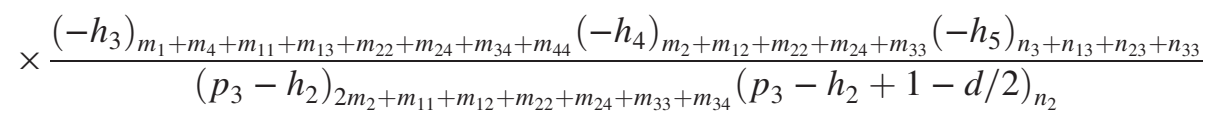

$$
\begin{aligned}
& \times \frac{\left(u_{1}^{7}\right)^{n_{1}+r}\left(u_{2}^{7}\right)^{n_{2}+r_{34}}\left(u_{3}^{7}\right)^{n_{3}+r_{56}}\left(u_{4}^{7}\right)^{m_{4}}}{n_{1} ! n_{2} ! n_{3} !} \prod_{1 \leq a \leq b \leq 4} \frac{\left(1-v_{a b}^{7}\right)^{m_{a b}}}{m_{a b} !} F_{6} .
\end{aligned}
$$

After shifting $n$ such that $n \rightarrow n+m_{11}+m_{22}+m_{24}+m_{34}+r+r_{34}-r_{56}$, we can evaluate the sum over $n$, giving

$$
\begin{aligned}
& G_{7}=\sum \frac{\left(-h_{6}\right)_{m_{4}+m_{14}+m_{24}+m_{34}+m_{44}}\left(p_{4}-h_{3}+h_{6}\right)_{m_{4}+m_{11}+m_{13}+m_{22}}}{\left(p_{4}-h_{3}\right)_{2 m_{4}+m_{11}+m_{13}+m_{14}+m_{22}+m_{24}+m_{34}+m_{44}}\left(p_{4}-h_{3}+1-d / 2\right)_{m_{4}}} \\
& \times \frac{\left(p_{4}-n_{1}\right)_{m_{4}+m_{14}-r}}{\left(m_{4}-r-k_{23}\right) ! r_{34} !\left(r-r_{56}-r_{34}-k_{12}-k_{33}\right) ! r_{56} ! k_{12} ! k_{23} ! k_{33} !} \\
& \times \frac{m_{33} !(-1)^{k_{12}+k_{23}+k_{33}}\left(-r_{56}-k_{12}\right)_{n_{13}-m_{13}-m_{44}-r_{56}-k_{23}-k_{33}}}{\left(n_{13}-m_{13}-m_{44}-r_{56}-k_{23}-k_{33}\right) ! n_{33} !} \\
& \times \frac{\left(p_{2}+h_{3}\right)_{n_{1}+n_{23}}\left(p_{3}-h_{2}+h_{4}\right)_{m_{2}+m_{11}+m_{34}}\left(\bar{p}_{3}+h_{2}+h_{5}\right)_{n_{3}+n_{12}}}{\left(\bar{p}_{3}+h_{2}\right)_{2 n_{3}+n_{12}+n_{13}+n_{23}+n_{33}\left(\bar{p}_{3}+h_{2}+1-d / 2\right)_{n_{3}}}} \\
& \times \frac{\left(p_{3}\right)_{n_{2}+n_{3}-n_{1}+n_{12}+n_{33}}\left(p_{2}+h_{2}\right)_{m_{1}-m_{2}+m_{3}+m_{13}+m_{23}+m_{44}}\left(-h_{2}\right)_{m_{1}+m_{2}-m_{3}+m_{11}+m_{22}+m_{24}+m_{34}}}{\left(p_{2}\right)_{2 m_{1}+m_{11}+m_{13}+m_{22}+m_{23}+m_{24}+m_{34}+m_{44}}\left(p_{2}+1-d / 2\right)_{n_{1}}}
\end{aligned}
$$

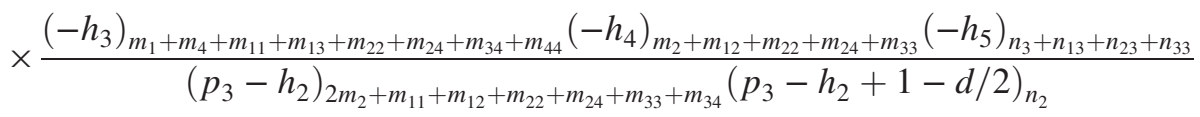

$$
\begin{aligned}
& \times \frac{\left(u_{1}^{7}\right)^{n_{1}+r}\left(u_{2}^{7}\right)^{n_{2}+r_{34}}\left(u_{3}^{7}\right)^{n_{3}+r_{56}}\left(u_{4}^{7}\right)^{m_{4}}}{n_{1} ! n_{2} ! n_{3} !} \prod_{1 \leq a \leq b \leq 4} \frac{\left(1-v_{a b}^{7}\right)^{m_{a b}}}{m_{a b} !} F_{6} .
\end{aligned}
$$

We then change variables again, this time $n_{13} \rightarrow n_{13}+m_{13}+m_{44}+r_{56}+k_{23}+k_{33}$, and sum over $n_{13}$, which leads to 


$$
\begin{aligned}
& G_{7}=\sum \frac{\left(-h_{6}\right)_{m_{4}+m_{14}+m_{24}+m_{34}+m_{44}}\left(p_{4}-h_{3}+h_{6}\right)_{m_{4}+m_{11}+m_{13}+m_{22}}}{\left(p_{4}-h_{3}\right)_{2 m_{4}+m_{11}+m_{13}+m_{14}+m_{22}+m_{24}+m_{34}+m_{44}}\left(p_{4}-h_{3}+1-d / 2\right)_{m_{4}}} \\
& \times \frac{\left(p_{4}-n_{1}\right)_{m_{4}+m_{14}-r}\left(-h_{5}\right)_{m_{3}+m_{13}+m_{23}+m_{33}+m_{44}}}{\left(m_{4}-r-k_{23}\right) ! r_{34} !\left(r-r_{56}-r_{34}-k_{12}-k_{33}\right) ! r_{56} ! k_{12} ! k_{23} ! k_{33} !} \\
& \times \frac{\left(p_{2}+h_{3}\right)_{n_{1}+n_{23}}\left(p_{3}-h_{2}+h_{4}\right)_{m_{2}+m_{11}+m_{34}}\left(\bar{p}_{3}+h_{2}+h_{5}\right)_{m_{3}+m_{12}}}{\left(\bar{p}_{3}+h_{2}\right)_{2 m_{3}+m_{12}+m_{13}+m_{23}+m_{33}+m_{44}}\left(\bar{p}_{3}+h_{2}+1-d / 2\right)_{n_{3}}} \\
& \times \frac{\left(p_{3}\right)_{n_{2}+n_{3}-n_{1}+n_{12}+n_{33}}\left(p_{2}+h_{2}\right)_{m_{1}-m_{2}+m_{3}+m_{13}+m_{23}+m_{44}}\left(-h_{2}\right)_{m_{1}+m_{2}-m_{3}+m_{11}+m_{22}+m_{24}+m_{34}}}{\left(p_{2}\right)_{2 m_{1}+m_{11}+m_{13}+m_{22}+m_{23}+m_{24}+m_{34}+m_{44}}\left(p_{2}+1-d / 2\right)_{n_{1}}}
\end{aligned}
$$

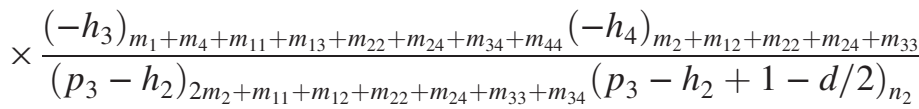

$$
\begin{aligned}
& \times \frac{m_{12} ! m_{23} ! m_{33} !(-1)^{k_{12}+k_{23}+k_{33}}}{n_{12} ! n_{23} ! n_{33} !} \frac{\left(u_{1}^{7}\right)^{n_{1}+r}\left(u_{2}^{7}\right)^{n_{2}+r_{34}}\left(u_{3}^{7}\right)^{n_{3}+r_{56}}\left(u_{4}^{7}\right)^{m_{4}}}{n_{1} ! n_{2} ! n_{3} !} \prod_{1 \leq a \leq b \leq 4} \frac{\left(1-v_{a b}^{7}\right)^{m_{a b}}}{m_{a b} !} F_{6} .
\end{aligned}
$$

Using the fact that $m_{12}=n_{12}+k_{12}, m_{23}=n_{13}+k_{23}$, and $m_{33}=k_{33}+n_{33}$, we evaluate the sums over $k_{12}, k_{23}$, and $k_{33}$, to obtain

$$
\begin{aligned}
& G_{7}=\sum \frac{\left(p_{4}-m_{1}\right)_{m_{4}+m_{14}}\left(-h_{6}\right)_{m_{4}+m_{14}+m_{24}+m_{34}+m_{44}}\left(p_{4}-h_{3}+h_{6}\right)_{m_{4}+m_{11}+m_{13}+m_{22}}}{\left(p_{2}+h_{3}+m_{1}\right)_{-m_{4}}\left(p_{4}-h_{3}\right)_{2 m_{4}+m_{11}+m_{13}+m_{14}+m_{22}+m_{24}+m_{34}+m_{44}}\left(p_{4}-h_{3}+1-d / 2\right)_{m_{4}}} \\
& \times \frac{\left(p_{2}+h_{3}\right)_{m_{1}-m_{4}+m_{23}}\left(p_{3}\right)_{-m_{1}+m_{2}+m_{3}+m_{12}+m_{33}}\left(p_{3}-h_{2}+h_{4}\right)_{m_{2}+m_{11}+m_{34}}\left(\bar{p}_{3}+h_{2}+h_{5}\right)_{m_{3}+m_{12}}}{\left(\bar{p}_{3}+h_{2}\right)_{2 m_{3}+m_{12}+m_{13}+m_{23}+m_{33}+m_{44}}\left(\bar{p}_{3}+h_{2}+1-d / 2\right)_{m_{3}}}
\end{aligned}
$$

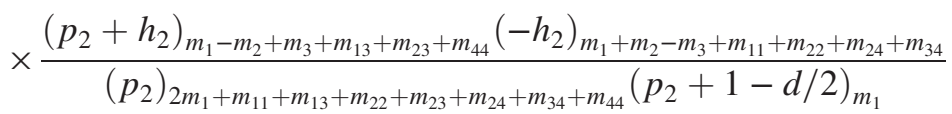

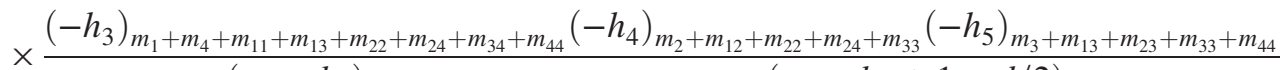

$$
\begin{aligned}
& \left(p_{3}-h_{2}\right)_{2 m_{2}+m_{11}+m_{12}+m_{22}+m_{24}+m_{33}+m_{34}}\left(p_{3}-h_{2}+1-d / 2\right)_{m_{2}} \\
& \times \frac{\left(-m_{1}\right)_{r}\left(-m_{4}\right)_{r}\left(-p_{2}+d / 2-m_{1}\right)_{r}\left(p_{3}-m_{1}+m_{2}+m_{3}\right)_{r-r_{34}-r_{56}}}{\left(1-p_{2}-h_{3}-m_{1}\right)_{r}\left(p_{4}-m_{1}\right)_{r}\left(r-r_{56}-r_{34}\right) !} \\
& \times \frac{\left(-m_{2}\right)_{r_{34}}\left(-m_{3}\right)_{r_{56}}\left(-p_{3}+h_{2}+d / 2-m_{2}\right)_{r_{34}}\left(-\bar{p}_{3}-h_{2}+d / 2-m_{3}\right)_{r_{56}}}{r_{34} ! r_{56} !} \\
& \times F_{6} \prod_{1 \leq a \leq 4} \frac{\left(u_{a}^{7}\right)^{m_{a}}}{m_{a} !} \prod_{1 \leq a \leq b \leq 4} \frac{\left(1-v_{a b}^{7}\right)^{m_{a b}}}{m_{a b} !} .
\end{aligned}
$$

Expanding $F_{6}$ with the help of the first result in (2.13) leads to

$$
\begin{aligned}
& G_{7}=\sum \frac{\left(p_{4}-m_{1}\right)_{m_{4}+m_{14}}\left(-h_{6}\right)_{m_{4}+m_{14}+m_{24}+m_{34}+m_{44}}\left(p_{4}-h_{3}+h_{6}\right)_{m_{4}+m_{11}+m_{13}+m_{22}}}{\left(p_{2}+h_{3}+m_{1}\right)_{-m_{4}}\left(p_{4}-h_{3}\right)_{2 m_{4}+m_{11}+m_{13}+m_{14}+m_{22}+m_{24}+m_{34}+m_{44}}\left(p_{4}-h_{3}+1-d / 2\right)_{m_{4}}} \\
& \times \frac{\left(p_{2}+h_{3}\right)_{m_{1}-m_{4}+m_{23}}\left(p_{3}\right)_{-m_{1}+m_{2}+m_{3}+m_{12}+m_{33}}\left(p_{3}-h_{2}+h_{4}\right)_{m_{2}+m_{11}+m_{34}}\left(\bar{p}_{3}+h_{2}+h_{5}\right)_{m_{3}+m_{12}}}{\left(\bar{p}_{3}+h_{2}\right)_{2 m_{3}+m_{12}+m_{13}+m_{23}+m_{33}+m_{44}}\left(\bar{p}_{3}+h_{2}+1-d / 2\right)_{m_{3}}} \\
& \times \frac{\left(p_{2}+h_{2}\right)_{m_{1}-m_{2}+m_{3}+m_{13}+m_{23}+m_{44}}\left(-h_{2}\right)_{m_{1}+m_{2}-m_{3}+m_{11}+m_{22}+m_{24}+m_{34}}}{\left(p_{2}\right)_{2 m_{1}+m_{11}+m_{13}+m_{22}+m_{23}+m_{24}+m_{34}+m_{44}\left(p_{2}+1-d / 2\right)_{m_{1}}}} \\
& \times \frac{\left(-h_{3}\right)_{m_{1}+m_{4}+m_{11}+m_{13}+m_{22}+m_{24}+m_{34}+m_{44}}\left(-h_{4}\right)_{m_{2}+m_{12}+m_{22}+m_{24}+m_{33}}\left(-h_{5}\right)_{m_{3}+m_{13}+m_{23}+m_{33}+m_{44}}}{\left(p_{3}-h_{2}\right)_{2}+m_{2}+m_{11}+m_{12}+m_{23}+m_{24}+m_{33}+m_{34}} \\
& \times F_{7} \prod_{1 \leq a \leq 4} \frac{\left(u_{a}^{7}\right)^{m_{a}}}{m_{a} !} \prod_{1 \leq a \leq b \leq 4} \frac{\left(1-v_{a b}^{7}\right)^{m_{a b}}}{m_{a b} !},
\end{aligned}
$$


which results in the appropriate $C_{7}$ presented in (2.17) and implies that $F_{7}$ is given by

$$
\begin{aligned}
F_{7}= & \frac{\left(-p_{3}+h_{2}+d / 2-m_{2}\right)_{m_{2}}}{\left(p_{3}\right)_{-m_{1}}\left(-h_{2}\right)_{-m_{3}}} \sum \frac{\left(-m_{2}\right)_{r_{34}+t_{1}}\left(-m_{3}\right)_{r_{56}+t_{2}}\left(-\bar{p}_{3}-h_{2}+d / 2-m_{3}\right)_{r_{56}}}{\left(r-r_{56}-r_{34}\right) ! r_{34} ! r_{56} !} \\
& \times \frac{\left(-m_{1}\right)_{r+t_{2}}\left(-m_{4}\right)_{r}\left(-p_{2}+d / 2-m_{1}\right)_{r}\left(p_{3}-m_{1}+m_{2}+m_{3}\right)_{r-r_{56}-r_{34}}}{\left(1-p_{2}-h_{3}-m_{1}\right)_{r}\left(p_{4}-m_{1}\right)_{r}} \\
& \times \frac{\left(-h_{2}\right)_{t_{1}}\left(p_{3}\right)_{t_{1}}}{\left(-h_{2}-m_{3}\right)_{r_{56}+t_{1}+t_{2}}\left(p_{3}-m_{1}\right)_{r+t_{1}+t_{2}}} \frac{\left.\left(\bar{t}_{3}-d / 2\right)_{t_{1}+t_{2}}+1-d / 2\right)_{t_{1}} t_{1} ! t_{2} !}{\left(p_{2}+\right.}
\end{aligned}
$$

To reach the solution introduced in (2.18), we first evaluate the sum over $r_{34}$, leading to

$$
\begin{aligned}
F_{7}= & \frac{\left(-p_{3}+h_{2}+d / 2-m_{2}\right)_{m_{2}}}{\left(p_{3}\right)_{-m_{1}}\left(-h_{2}\right)_{-m_{3}}} \sum \frac{\left(-m_{2}\right)_{t_{1}}\left(-m_{3}\right)_{r_{56}+t_{2}}\left(-\bar{p}_{3}-h_{2}+d / 2-m_{3}\right)_{r_{56}}}{\left(r-r_{56}\right) ! r_{56} !} \\
& \times \frac{\left(-m_{1}\right)_{r+t_{2}}\left(-m_{4}\right)_{r}\left(-p_{2}+d / 2-m_{1}\right)_{r}\left(p_{3}-m_{1}+m_{3}+t_{1}\right)_{r-r_{56}}}{\left(1-p_{2}-h_{3}-m_{1}\right)_{r}\left(p_{4}-m_{1}\right)_{r}} \\
& \times \frac{\left(-h_{2}\right)_{t_{1}}\left(p_{3}\right)_{t_{1}}}{\left(-h_{2}-m_{3}\right)_{r_{56}+t_{1}+t_{2}}\left(p_{3}-m_{1}\right)_{r+t_{1}+t_{2}}} \frac{\left(p_{3}-h_{2}+1-d / 2\right)_{t_{1}} t_{1} ! t_{2} !}{\left(p_{3} !\right.}
\end{aligned}
$$

We then use the first identity in (A.3) and get

$$
\begin{aligned}
F_{7}= & \frac{\left(-p_{3}+h_{2}+d / 2-m_{2}\right)_{m_{2}}}{\left(p_{3}\right)_{-m_{1}}\left(-h_{2}\right)_{-m_{3}}} \sum \frac{\left(-m_{2}\right)_{t_{1}}\left(-m_{3}\right)_{r_{56}+t_{2}}\left(-\bar{p}_{3}-h_{2}+d / 2-m_{3}\right)_{m_{3}}}{\left(r-r_{56}\right) ! r_{56} !} \\
& \times \frac{\left(-m_{1}\right)_{r}\left(-m_{4}\right)_{r}\left(-p_{2}+d / 2-m_{1}\right)_{r}\left(p_{3}-m_{1}+m_{3}+t_{1}\right)_{r-r_{56}}}{\left(1-p_{2}-h_{3}-m_{1}\right)_{r}\left(p_{4}-m_{1}\right)_{r}\left(\bar{p}_{3}+h_{2}+1-d / 2\right)_{t_{2}}} \\
& \times \frac{\left(\bar{p}_{3}-d / 2\right)_{t_{1}+t_{2}}}{\left(-h_{2}-m_{3}\right)_{m_{3}+t_{1}}\left(p_{3}-m_{1}\right)_{r+t_{1}+t_{2}}} \frac{\left(-h_{2}\right)_{t_{1}}\left(p_{3}\right)_{t_{1}+t_{2}}}{\left(p_{3}-h_{2}+1-d / 2\right)_{t_{1}} t_{1} ! t_{2} !} .
\end{aligned}
$$

We finally sum over $r_{56}$, leading to (2.18) with the second equality for $F_{6}$ in (2.13), which concludes our proof of the scalar seven-point conformal blocks in the extended snowflake channel.

\section{APPENDIX B: SYMMETRY PROPERTIES}

This Appendix is dedicated to the proofs of the symmetry properties of the scalar seven-point conformal blocks in the extended snowflake channel. The three generators to investigate are the reflections as well as the dendrite permutations of the first and second kinds introduced in (3.1), (3.2), and (3.3), respectively.

\section{Reflections}

We aim to prove (3.1), which corresponds to the invariance of the scalar seven-point conformal blocks under reflections. For simplicity, we denote (3.1) by $G_{7}=G_{7 R}$. We first rewrite the conformal cross-ratios in (3.1) in terms of the original conformal cross-ratios and then resum the extra sums to obtain $G_{7}$ back, taking into account that $F_{7}$ is invariant under reflections as dictated by (2.18). 
We therefore start with

$$
\begin{aligned}
& G_{7 R}=\sum \frac{\left(p_{4}-m_{1}^{\prime}\right)_{m_{4}^{\prime}+m_{14}}\left(-h_{6}\right)_{m_{4}^{\prime}+m_{14}+m_{24}+m_{34}+m_{44}}\left(p_{4}-h_{3}+h_{6}\right)_{m_{4}^{\prime}+m_{11}+m_{13}+m_{22}}}{\left(p_{2}+h_{3}+m_{1}^{\prime}\right)_{-m_{4}^{\prime}}\left(p_{4}-h_{3}\right)_{2 m_{4}^{\prime}+m_{11}+m_{13}+m_{14}+m_{22}+m_{24}+m_{34}+m_{44}}\left(p_{4}-h_{3}+1-d / 2\right)_{m_{4}^{\prime}}} \\
& \times \frac{\left(p_{2}+h_{3}\right)_{m_{1}^{\prime}-m_{4}^{\prime}+m_{23}}\left(p_{3}\right)_{-m_{1}^{\prime}+m_{2}^{\prime}+m_{3}^{\prime}+m_{12}+m_{33}}\left(\bar{p}_{3}+h_{2}+h_{5}\right)_{m_{3}^{\prime}+m_{11}+m_{34}}\left(p_{3}-h_{2}+h_{4}\right)_{m_{2}^{\prime}+m_{12}}}{\left(p_{3}-h_{2}\right)_{2 m_{2}^{\prime}+m_{12}+m_{13}+m_{23}+m_{33}+m_{44}}\left(p_{3}-h_{2}+1-d / 2\right)_{m_{2}^{\prime}}} \\
& \times \frac{\left(-h_{2}\right)_{m_{1}^{\prime}+m_{2}^{\prime}-m_{3}^{\prime}+m_{13}+m_{23}+m_{44}}\left(p_{2}+h_{2}\right)_{m_{1}^{\prime}-m_{2}^{\prime}+m_{3}^{\prime}+m_{11}+m_{22}+m_{24}+m_{34}}}{\left(p_{2}\right)_{2 m_{1}^{\prime}+m_{11}+m_{13}+m_{22}+m_{23}+m_{24}+m_{34}+m_{44}}\left(p_{2}+1-d / 2\right)_{m_{1}^{\prime}}} \\
& \times \frac{\left(-h_{3}\right)_{m_{1}^{\prime}+m_{4}^{\prime}+m_{11}+m_{13}+m_{22}+m_{24}+m_{34}+m_{44}}\left(-h_{5}\right)_{m_{3}^{\prime}+m_{12}+m_{22}+m_{24}+m_{33}}\left(-h_{4}\right)_{m_{2}^{\prime}+m_{13}+m_{23}+m_{33}+m_{44}}}{\left(\bar{p}_{3}+h_{2}\right)_{2 m_{3}^{\prime}+m_{11}+m_{12}+m_{22}+m_{24}+m_{33}+m_{34}}\left(\bar{p}_{3}+h_{2}+1-d / 2\right)_{m_{3}^{\prime}}} \\
& \times \prod_{1 \leq a \leq b \leq 4}\left(\begin{array}{c}
m_{a b} \\
k_{a b}
\end{array}\right)\left(\begin{array}{c}
h_{3}-h_{6}-m_{1}^{\prime}-k_{11}-k_{13}+k_{14}-k_{22} \\
m_{11}^{\prime}
\end{array}\right)\left(\begin{array}{c}
h_{4}-m_{2}^{\prime}-k_{13}-k_{23}-k_{33}-k_{44} \\
m_{12}^{\prime}
\end{array}\right) \\
& \times\left(\begin{array}{c}
k_{22} \\
m_{13}^{\prime}
\end{array}\right)\left(\begin{array}{c}
k_{14} \\
m_{14}^{\prime}
\end{array}\right)\left(\begin{array}{c}
k_{13} \\
m_{22}^{\prime}
\end{array}\right)\left(\begin{array}{c}
h_{5}-m_{3}^{\prime}-k_{12}-k_{22}-k_{24}-k_{33} \\
m_{23}^{\prime}
\end{array}\right)\left(\begin{array}{c}
k_{44} \\
m_{24}^{\prime}
\end{array}\right)\left(\begin{array}{c}
k_{33} \\
m_{33}^{\prime}
\end{array}\right) \\
& \times\left(\begin{array}{c}
h_{6}-m_{4}^{\prime}-k_{14}-k_{24}-k_{34}-k_{44} \\
m_{34}^{\prime}
\end{array}\right)\left(\begin{array}{c}
k_{24} \\
m_{44}^{\prime}
\end{array}\right)(-1)^{\sum_{1 \leq a \leq b \leq 4}\left(k_{a b}+m_{a b}^{\prime}\right)} F_{7} \prod_{1 \leq a \leq 4} \frac{\left(u_{a}^{7}\right)^{m_{a}^{\prime}}}{m_{a}^{\prime} !} \prod_{1 \leq a \leq b \leq 4} \frac{\left(1-v_{a b}^{7}\right)^{m_{a b}^{\prime}}}{m_{a b} !},
\end{aligned}
$$

and evaluate the sums over $k_{11}, k_{12}, k_{23}$ and $k_{34}$ to get

$$
\begin{aligned}
& G_{7 R}=\sum \frac{\left(p_{4}-m_{1}^{\prime}\right)_{m_{4}^{\prime}+m_{14}}\left(-h_{6}\right)_{m_{4}^{\prime}+m_{14}+m_{24}+m_{34}+m_{44}}\left(p_{4}-h_{3}+h_{6}\right)_{m_{4}^{\prime}+m_{11}+m_{13}+m_{22}}}{\left(p_{2}+h_{3}+m_{1}^{\prime}\right)_{-m_{4}^{\prime}}\left(p_{4}-h_{3}\right)_{2 m_{4}^{\prime}+m_{11}+m_{13}+m_{14}+m_{22}+m_{24}+m_{34}+m_{44}}\left(p_{4}-h_{3}+1-d / 2\right)_{m_{4}^{\prime}}} \\
& \times \frac{\left(p_{2}+h_{3}\right)_{m_{1}^{\prime}-m_{4}^{\prime}+m_{23}}\left(p_{3}\right)_{-m_{1}^{\prime}+m_{2}^{\prime}+m_{3}^{\prime}+m_{12}+m_{33}}\left(\bar{p}_{3}+h_{2}+h_{5}\right)_{m_{3}^{\prime}+m_{11}+m_{34}}\left(p_{3}-h_{2}+h_{4}\right)_{m_{2}^{\prime}+m_{12}}}{\left(p_{3}-h_{2}\right)_{2 m_{2}^{\prime}+m_{12}+m_{13}+m_{23}+m_{33}+m_{44}}\left(p_{3}-h_{2}+1-d / 2\right)_{m_{2}^{\prime}}}
\end{aligned}
$$

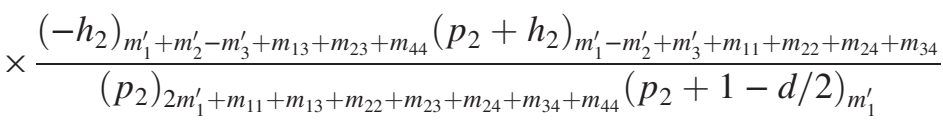

$$
\begin{aligned}
& \times \frac{\left(-h_{3}\right)_{m_{1}^{\prime}+m_{4}^{\prime}+m_{11}+m_{13}+m_{22}+m_{24}+m_{34}+m_{44}}\left(-h_{5}\right)_{m_{3}^{\prime}+m_{12}+m_{22}+m_{24}+m_{33}}\left(-h_{4}\right)_{m_{2}^{\prime}+m_{13}+m_{23}+m_{33}+m_{44}}}{\left(\bar{p}_{3}+h_{2}\right)_{2 m_{3}^{\prime}+m_{11}+m_{12}+m_{22}+m_{24}+m_{33}+m_{34}}\left(\bar{p}_{3}+h_{2}+1-d / 2\right)_{m_{3}^{\prime}}} \\
& \times\left(\begin{array}{c}
m_{13} \\
k_{13}
\end{array}\right)\left(\begin{array}{c}
m_{14} \\
k_{14}
\end{array}\right)\left(\begin{array}{c}
m_{22} \\
k_{22}
\end{array}\right)\left(\begin{array}{c}
m_{24} \\
k_{24}
\end{array}\right)\left(\begin{array}{c}
m_{33} \\
k_{33}
\end{array}\right)\left(\begin{array}{c}
m_{44} \\
k_{44}
\end{array}\right)\left(\begin{array}{c}
k_{22} \\
m_{13}^{\prime}
\end{array}\right)\left(\begin{array}{c}
k_{14} \\
m_{14}^{\prime}
\end{array}\right)\left(\begin{array}{c}
k_{13} \\
m_{22}^{\prime}
\end{array}\right)\left(\begin{array}{c}
k_{44} \\
m_{24}^{\prime}
\end{array}\right)\left(\begin{array}{c}
k_{33} \\
m_{33}^{\prime}
\end{array}\right)\left(\begin{array}{c}
k_{24} \\
m_{44}^{\prime}
\end{array}\right)
\end{aligned}
$$

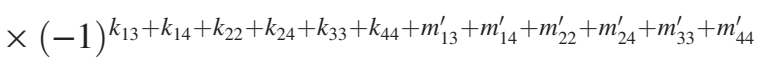

$$
\begin{aligned}
& \times \frac{\left(-m_{11}^{\prime}\right)_{m_{11}}\left(-h_{3}+h_{6}+m_{1}^{\prime}+m_{11}+k_{13}-k_{14}+k_{22}\right)_{m_{11}^{\prime}-m_{11}}}{m_{11}^{\prime} !} \\
& \times \frac{\left(-m_{23}^{\prime}\right)_{m_{12}}\left(-h_{5}+m_{3}^{\prime}+m_{12}+k_{22}+k_{24}+k_{33}\right)_{m_{23}^{\prime}-m_{12}}}{m_{23}^{\prime} !} \\
& \times \frac{\left(-m_{12}^{\prime}\right)_{m_{23}}\left(-h_{4}+m_{2}^{\prime}+m_{23}+k_{13}+k_{33}+k_{44}\right)_{m_{12}^{\prime}-m_{23}}}{m_{12}^{\prime} !} \\
& \times \frac{\left(-m_{34}^{\prime}\right)_{m_{34}}\left(-h_{6}+m_{4}^{\prime}+m_{34}+k_{14}+k_{24}+k_{44}\right)_{m_{34}^{\prime}-m_{34}}}{m_{34}^{\prime} !} \\
& \times F_{7} \prod_{1 \leq a \leq 4} \frac{\left(u_{a}^{7}\right)^{m_{a}^{\prime}}}{m_{a}^{\prime} !} \prod_{1 \leq a \leq b \leq 4} \frac{\left(1-v_{a b}^{7}\right)^{m_{a b}^{\prime}}}{m_{a b} !} .
\end{aligned}
$$


We then change variables by

$$
\begin{aligned}
& k_{13} \rightarrow k_{13}+m_{22}^{\prime}, \quad k_{14} \rightarrow k_{14}+m_{14}^{\prime}, \quad k_{22} \rightarrow k_{22}+m_{13}^{\prime}, \\
& k_{24} \rightarrow k_{24}+m_{44}^{\prime}, \quad k_{33} \rightarrow k_{33}+m_{33}^{\prime}, \quad k_{44} \rightarrow k_{44}+m_{24}^{\prime} \text {, }
\end{aligned}
$$

and use the following identity:

$$
\begin{aligned}
& \left(-h_{3}+h_{6}+m_{1}^{\prime}+m_{13}^{\prime}-m_{14}^{\prime}+m_{22}^{\prime}+m_{11}+k_{13}-k_{14}+k_{22}\right)_{m_{11}^{\prime}-m_{11}} \\
& \quad=\sum_{j_{1} \geq 0}\left(\begin{array}{c}
m_{11}^{\prime}-m_{11} \\
j_{1}
\end{array}\right)\left(-m_{13}+m_{22}^{\prime}+k_{13}\right)_{j_{1}}\left(-h_{3}+h_{6}+m_{1}^{\prime}+m_{13}^{\prime}-m_{14}^{\prime}+m_{11}+m_{13}-k_{14}+k_{22}\right)_{m_{11}^{\prime}-m_{11}-j_{1}},
\end{aligned}
$$

to compute the sum over $k_{13}$. With the help of a similar trick, we evaluate the sums over all other $k_{a b}$ 's except $k_{14}$, to get

$$
\begin{aligned}
& G_{7 R}=\sum \frac{\left(p_{4}-m_{1}^{\prime}\right)_{m_{4}^{\prime}+m_{14}}\left(-h_{6}\right)_{m_{4}^{\prime}+m_{14}+m_{24}+m_{34}+m_{44}}\left(p_{4}-h_{3}+h_{6}\right)_{m_{4}^{\prime}+m_{11}+m_{13}+m_{22}}}{\left(p_{2}+h_{3}+m_{1}^{\prime}\right)_{-m_{4}^{\prime}}\left(p_{4}-h_{3}\right)_{2 m_{4}^{\prime}+m_{11}+m_{13}+m_{14}+m_{22}+m_{24}+m_{34}+m_{44}}\left(p_{4}-h_{3}+1-d / 2\right)_{m_{4}^{\prime}}} \\
& \times \frac{\left(p_{2}+h_{3}\right)_{m_{1}^{\prime}-m_{4}^{\prime}+m_{23}}\left(p_{3}\right)_{-m_{1}^{\prime}+m_{2}^{\prime}+m_{3}^{\prime}+m_{12}+m_{33}}\left(\bar{p}_{3}+h_{2}+h_{5}\right)_{m_{3}^{\prime}+m_{11}+m_{34}}\left(p_{3}-h_{2}+h_{4}\right)_{m_{2}^{\prime}+m_{12}}}{\left(p_{3}-h_{2}\right)_{2 m_{2}^{\prime}+m_{12}+m_{13}+m_{23}+m_{33}+m_{44}}\left(p_{3}-h_{2}+1-d / 2\right)_{m_{2}^{\prime}}} \\
& \times \frac{\left(-h_{2}\right)_{m_{1}^{\prime}+m_{2}^{\prime}-m_{3}^{\prime}+m_{13}+m_{23}+m_{44}}\left(p_{2}+h_{2}\right)_{m_{1}^{\prime}-m_{2}^{\prime}+m_{3}^{\prime}+m_{11}+m_{22}+m_{24}+m_{34}}}{\left(p_{2}\right)_{2 m_{1}^{\prime}+m_{11}+m_{13}+m_{22}+m_{23}+m_{24}+m_{34}+m_{44}\left(p_{2}+1-d / 2\right)_{m_{1}^{\prime}}}}
\end{aligned}
$$

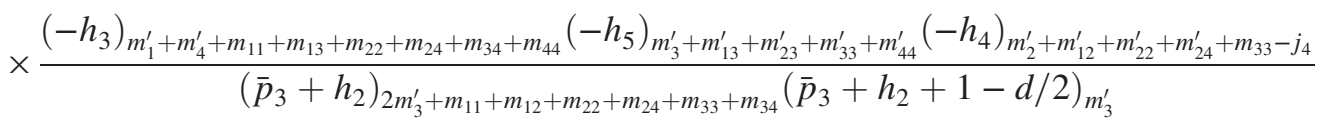

$$
\begin{aligned}
& \times \frac{\left(-m_{11}^{\prime}\right)_{m_{11}+j_{1}+j_{2}}\left(-h_{3}+h_{6}+m_{1}^{\prime}-m_{14}^{\prime}+m_{11}+m_{13}+m_{22}-k_{14}\right)_{m_{11}^{\prime}-m_{11}-j_{1}-j_{2}}}{\left(m_{22}-m_{13}^{\prime}-j_{2}\right) !\left(m_{13}-m_{22}^{\prime}-j_{1}\right) !} \\
& \times \frac{\left(-m_{23}^{\prime}\right)_{m_{12}+m_{22}+m_{24}+m_{33}-m_{13}^{\prime}-m_{33}^{\prime}-m_{44}^{\prime}-j_{2}-j_{3}-j_{4}}}{\left(-h_{5}+m_{3}^{\prime}+m_{12}+m_{22}+m_{24}+m_{33}\right)_{-j_{2}-j_{3}-j_{4}}\left(m_{24}-m_{44}^{\prime}-j_{3}\right) !\left(m_{44}-m_{24}^{\prime}-j_{5}\right) !} \\
& \times \frac{\left(-m_{34}^{\prime}\right)_{m_{34}+j_{3}+j_{5}}\left(-m_{12}^{\prime}\right)_{m_{13}+m_{23}+m_{44}-m_{22}^{\prime}-m_{24}^{\prime}-j_{1}+j_{4}-j_{5}}}{\left(-h_{4}+m_{2}^{\prime}+m_{13}+m_{23}+m_{33}+m_{44}\right)_{-j_{1}-j_{5}} j_{1} ! j_{2} ! j_{3} ! j_{4} ! j_{5} !\left(m_{33}-m_{33}^{\prime}-j_{4}\right) !} \\
& \times(-1)^{k_{14}} \frac{\left(-h_{6}+m_{4}^{\prime}+m_{14}^{\prime}+m_{24}+m_{34}+m_{44}+k_{14}\right)_{m_{34}^{\prime}-m_{34}-j_{3}-j_{5}}}{\left(m_{14}-m_{14}^{\prime}-k_{14}\right) ! k_{14} ! m_{11} ! m_{12} ! m_{23} ! m_{34} !} \\
& \times F_{7} \prod_{1 \leq a \leq 4} \frac{\left(u_{a}^{7}\right)^{m_{a}^{\prime}}}{m_{a}^{\prime} !} \prod_{1 \leq a \leq b \leq 4} \frac{\left(1-v_{a b}^{7}\right)^{m_{a b}^{\prime}}}{m_{a b}^{\prime} !} .
\end{aligned}
$$

We now use the identity,

$$
\begin{aligned}
& \left(-h_{3}+h_{6}+m_{1}^{\prime}-m_{14}^{\prime}+m_{11}+m_{13}+m_{22}-k_{14}\right)_{m_{11}^{\prime}-m_{11}-j_{1}-j_{2}} \\
& \quad=\sum_{j_{6} \geq 0}\left(\begin{array}{c}
m_{11}^{\prime}-m_{11}-j_{1}-j_{2} \\
j_{6}
\end{array}\right)\left(-k_{14}\right)_{j_{6}}\left(-h_{3}+h_{6}+m_{1}^{\prime}-m_{14}^{\prime}+m_{11}+m_{13}+m_{22}\right)_{m_{11}^{\prime}-m_{11}-j_{1}-j_{2}-j_{6}},
\end{aligned}
$$

and then change $k_{14}$ by $k_{14} \rightarrow k_{14}+j_{6}$ to resum over $k_{14}$. This procedure leads to 


$$
\begin{aligned}
& G_{7 R}=\sum \frac{\left(p_{4}-m_{1}^{\prime}\right)_{m_{4}^{\prime}+m_{14}}\left(-h_{6}\right)_{m_{4}^{\prime}+m_{14}^{\prime}+m_{24}+m_{34}^{\prime}+m_{44}-j_{3}-j_{5}+j_{6}}\left(p_{4}-h_{3}+h_{6}\right)_{m_{4}^{\prime}+m_{11}+m_{13}+m_{22}}}{\left(p_{2}+h_{3}+m_{1}^{\prime}\right)_{-m_{4}^{\prime}}\left(p_{4}-h_{3}\right)_{2 m_{4}^{\prime}+m_{11}+m_{13}+m_{14}+m_{22}+m_{24}+m_{34}+m_{44}}\left(p_{4}-h_{3}+1-d / 2\right)_{m_{4}^{\prime}}} \\
& \times \frac{\left(p_{2}+h_{3}\right)_{m_{1}^{\prime}-m_{4}^{\prime}+m_{23}}\left(p_{3}\right)_{-m_{1}^{\prime}+m_{2}^{\prime}+m_{3}^{\prime}+m_{12}+m_{33}}\left(\bar{p}_{3}+h_{2}+h_{5}\right)_{m_{3}^{\prime}+m_{11}+m_{34}}\left(p_{3}-h_{2}+h_{4}\right)_{m_{2}^{\prime}+m_{12}}}{\left(p_{3}-h_{2}\right)_{2 m_{2}^{\prime}+m_{12}+m_{13}+m_{23}+m_{33}+m_{44}}\left(p_{3}-h_{2}+1-d / 2\right)_{m_{2}^{\prime}}}
\end{aligned}
$$

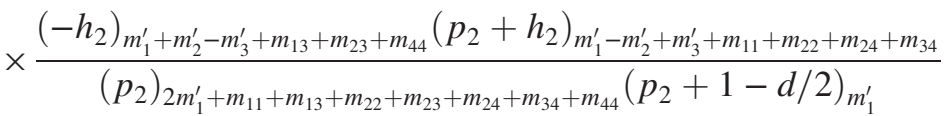

$$
\begin{aligned}
& \times \frac{\left(-h_{3}\right)_{m_{1}^{\prime}+m_{4}^{\prime}+m_{11}+m_{13}+m_{22}+m_{24}+m_{34}+m_{44}}\left(-h_{5}\right)_{m_{3}^{\prime}+m_{13}^{\prime}+m_{23}^{\prime}+m_{33}^{\prime}+m_{44}^{\prime}}\left(-h_{4}\right)_{m_{2}^{\prime}+m_{12}^{\prime}+m_{22}^{\prime}+m_{24}^{\prime}+m_{33}-j_{4}}}{\left(\bar{p}_{3}+h_{2}\right)_{2 m_{3}^{\prime}+m_{11}+m_{12}+m_{22}+m_{24}+m_{33}+m_{34}}\left(\bar{p}_{3}+h_{2}+1-d / 2\right)_{m_{3}^{\prime}}} \\
& \times \frac{\left(-m_{11}^{\prime}\right)_{m_{11}+j_{1}+j_{2}+j_{6}}\left(-h_{3}+h_{6}+m_{1}^{\prime}-m_{14}^{\prime}+m_{11}+m_{13}+m_{22}\right)_{m_{11}^{\prime}-m_{11}-j_{1}-j_{2}-j_{6}}}{\left(m_{22}-m_{13}^{\prime}-j_{2}\right) !\left(m_{13}-m_{22}^{\prime}-j_{1}\right) !} \\
& \times \frac{\left(-m_{23}^{\prime}\right)_{m_{12}+m_{22}+m_{24}+m_{33}-m_{13}^{\prime}-m_{33}^{\prime}-m_{44}^{\prime}-j_{2}-j_{3}-j_{4}}}{\left(-h_{5}+m_{3}^{\prime}+m_{12}+m_{22}+m_{24}+m_{33}\right)_{-j_{2}-j_{3}-j_{4}}\left(m_{24}-m_{44}^{\prime}-j_{3}\right) !\left(m_{44}-m_{24}^{\prime}-j_{5}\right) !} \\
& \times \frac{\left(-m_{34}^{\prime}\right)_{m_{14}+m_{34}-m_{14}^{\prime}+j_{3}+j_{5}-j_{6}}\left(-m_{12}^{\prime}\right)_{m_{13}+m_{23}+m_{44}-m_{22}^{\prime}-m_{24}^{\prime}-j_{1}+j_{4}-j_{5}}}{\left(-h_{4}+m_{2}^{\prime}+m_{13}+m_{23}+m_{33}+m_{44}\right)_{-j_{1}-j_{5}} j_{1} ! j_{2} ! j_{3} ! j_{4} ! j_{5} ! j_{6} !\left(m_{33}-m_{33}^{\prime}-j_{4}\right) !} \\
& \times \frac{(-1)^{j_{6}}}{\left(m_{14}-m_{14}^{\prime}-j_{6}\right) ! m_{11} ! m_{12} ! m_{23} ! m_{34} !} F_{7} \prod_{1 \leq a \leq 4} \frac{\left(u_{a}^{7}\right)^{m_{a}^{\prime}}}{m_{a}^{\prime} !} \prod_{1 \leq a \leq b \leq 4} \frac{\left(1-v_{a b}^{7}\right)^{m_{a b}^{\prime}}}{m_{a b}^{\prime} !} .
\end{aligned}
$$

It is thus possible to evaluate the sum over $m_{14}$ (after shifting $m_{14}$ by $m_{14}+m_{14}^{\prime}+j_{6}$ ) such that

$$
\begin{aligned}
& G_{7 R}=\sum \frac{\left(p_{4}-m_{1}^{\prime}\right)_{m_{4}^{\prime}+m_{14}^{\prime}+j_{6}}\left(-h_{6}\right)_{m_{4}^{\prime}+m_{14}^{\prime}+m_{24}+m_{34}^{\prime}+m_{44}-j_{3}-j_{5}+j_{6}}\left(p_{4}-h_{3}+h_{6}\right)_{m_{4}^{\prime}+m_{11}+m_{13}+m_{22}}}{\left(p_{2}+h_{3}+m_{1}^{\prime}\right)_{-m_{4}^{\prime}}\left(p_{4}-h_{3}\right)_{2 m_{4}^{\prime}+m_{11}+m_{13}+m_{14}^{\prime}+m_{22}+m_{24}+m_{34}^{\prime}+m_{44}-j_{3}-j_{5}+j_{6}}\left(p_{4}-h_{3}+1-d / 2\right)_{m_{4}^{\prime}}} \\
& \times \frac{\left(p_{2}+h_{3}\right)_{m_{1}^{\prime}-m_{4}^{\prime}+m_{23}}\left(p_{3}\right)_{-m_{1}^{\prime}+m_{2}^{\prime}+m_{3}^{\prime}+m_{12}+m_{33}}\left(\bar{p}_{3}+h_{2}+h_{5}\right)_{m_{3}^{\prime}+m_{11}+m_{34}}\left(p_{3}-h_{2}+h_{4}\right)_{m_{2}^{\prime}+m_{12}}}{\left(p_{3}-h_{2}\right)_{2 m_{2}^{\prime}+m_{12}+m_{13}+m_{23}+m_{33}+m_{44}}\left(p_{3}-h_{2}+1-d / 2\right)_{m_{2}^{\prime}}} \\
& \times \frac{\left(-h_{2}\right)_{m_{1}^{\prime}+m_{2}^{\prime}-m_{3}^{\prime}+m_{13}+m_{23}+m_{44}}\left(p_{2}+h_{2}\right)_{m_{1}^{\prime}-m_{2}^{\prime}+m_{3}^{\prime}+m_{11}+m_{22}+m_{24}+m_{34}}}{\left(p_{2}\right)_{2 m_{1}^{\prime}+m_{11}+m_{13}+m_{22}+m_{23}+m_{24}+m_{34}+m_{44}}\left(p_{2}+1-d / 2\right)_{m_{1}^{\prime}}}
\end{aligned}
$$

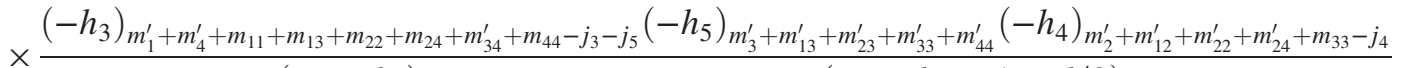

$$
\begin{aligned}
& \left(\bar{p}_{3}+h_{2}\right)_{2 m_{3}^{\prime}+m_{11}+m_{12}+m_{22}+m_{24}+m_{33}+m_{34}}\left(\bar{p}_{3}+h_{2}+1-d / 2\right)_{m_{3}^{\prime}} \\
& \times \frac{\left(-m_{11}^{\prime}\right)_{m_{11}+j_{1}+j_{2}+j_{6}}\left(-h_{3}+h_{6}+m_{1}^{\prime}-m_{14}^{\prime}+m_{11}+m_{13}+m_{22}\right)_{m_{11}^{\prime}-m_{11}-j_{1}-j_{2}-j_{6}}}{\left(m_{22}-m_{13}^{\prime}-j_{2}\right) !\left(m_{13}-m_{22}^{\prime}-j_{1}\right) !} \\
& \times \frac{\left(-m_{23}^{\prime}\right)_{m_{12}+m_{22}+m_{24}+m_{33}-m_{13}^{\prime}-m_{33}^{\prime}-m_{44}^{\prime}-j_{2}-j_{3}-j_{4}}}{\left(-h_{5}+m_{3}^{\prime}+m_{12}+m_{22}+m_{24}+m_{33}\right)_{-j_{2}-j_{3}-j_{4}}\left(m_{24}-m_{44}^{\prime}-j_{3}\right) !\left(m_{44}-m_{24}^{\prime}-j_{5}\right) !} \\
& \times \frac{\left(-m_{34}^{\prime}\right)_{m_{34}+j_{3}+j_{5}}\left(-m_{12}^{\prime}\right)_{m_{13}+m_{23}+m_{44}-m_{22}^{\prime}-m_{24}^{\prime}-j_{1}+j_{4}-j_{5}}}{\left(-h_{4}+m_{2}^{\prime}+m_{13}+m_{23}+m_{33}+m_{44}\right)_{-j_{1}-j_{5}} j_{1} ! j_{2} ! j_{3} ! j_{4} ! j_{5} ! j_{6} !\left(m_{33}-m_{33}^{\prime}-j_{4}\right) !} \\
& \times \frac{(-1)^{j_{6}}}{m_{11} ! m_{12} ! m_{23} ! m_{34} !} F_{7} \prod_{1 \leq a \leq 4} \frac{\left(u_{a}^{7}\right)^{m_{a}^{\prime}}}{m_{a}^{\prime} !} \prod_{1 \leq a \leq b \leq 4} \frac{\left(1-v_{a b}^{7}\right)^{m_{a b}^{\prime}}}{m_{a b}^{\prime} !} .
\end{aligned}
$$


With the help of the second identity in (A.3), we now eliminate the sum over $j_{6}$ and get

$$
\begin{aligned}
& G_{7 R}=\sum \frac{\left(p_{4}-m_{1}^{\prime}\right)_{m_{4}^{\prime}+m_{14}^{\prime}}\left(-h_{6}\right)_{m_{4}^{\prime}+m_{14}^{\prime}+m_{24}+m_{34}^{\prime}+m_{44}-j_{3}-j_{5}}\left(p_{4}-h_{3}+h_{6}\right)_{m_{4}^{\prime}+m_{11}^{\prime}+m_{13}+m_{22}-j_{1}-j_{2}}}{\left(p_{2}+h_{3}+m_{1}^{\prime}\right)_{-m_{4}^{\prime}}\left(p_{4}-h_{3}\right)_{2 m_{4}^{\prime}+m_{11}^{\prime}+m_{13}+m_{14}^{\prime}+m_{22}+m_{24}+m_{34}^{\prime}+m_{44}-j_{1}-j_{2}-j_{3}-j_{5}}\left(p_{4}-h_{3}+1-d / 2\right)_{m_{4}^{\prime}}} \\
& \times \frac{\left(p_{2}+h_{3}\right)_{m_{1}^{\prime}-m_{4}^{\prime}+m_{23}}\left(p_{3}\right)_{-m_{1}^{\prime}+m_{2}^{\prime}+m_{3}^{\prime}+m_{12}+m_{33}}\left(\bar{p}_{3}+h_{2}+h_{5}\right)_{m_{3}^{\prime}+m_{11}+m_{34}}\left(p_{3}-h_{2}+h_{4}\right)_{m_{2}^{\prime}+m_{12}}}{\left(p_{3}-h_{2}\right)_{2 m_{2}^{\prime}+m_{12}+m_{13}+m_{23}+m_{33}+m_{44}}\left(p_{3}-h_{2}+1-d / 2\right)_{m_{2}^{\prime}}}
\end{aligned}
$$

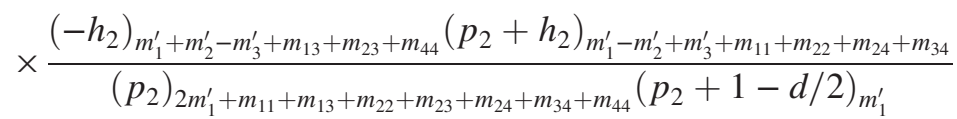

$$
\begin{aligned}
& \times \frac{\left(-h_{3}\right)_{m_{1}^{\prime}+m_{4}^{\prime}+m_{11}^{\prime}+m_{13}+m_{22}+m_{24}+m_{34}^{\prime}+m_{44}-j_{1}-j_{2}-j_{3}-j_{5}}\left(-h_{5}\right)_{m_{3}^{\prime}+m_{13}^{\prime}+m_{23}^{\prime}+m_{33}^{\prime}+m_{44}^{\prime}}}{\left(\bar{p}_{3}+h_{2}\right)_{2 m_{3}^{\prime}+m_{11}+m_{12}+m_{22}+m_{24}+m_{33}+m_{34}}\left(\bar{p}_{3}+h_{2}+1-d / 2\right)_{m_{3}^{\prime}}}
\end{aligned}
$$

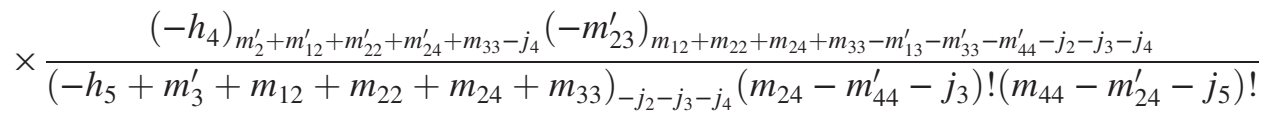

$$
\begin{aligned}
& \times \frac{\left(-m_{34}^{\prime}\right)_{m_{34}+j_{3}+j_{5}}\left(-m_{12}^{\prime}\right)_{m_{13}+m_{23}+m_{44}-m_{22}^{\prime}-m_{24}^{\prime}-j_{1}+j_{4}-j_{5}}}{\left(-h_{4}+m_{2}^{\prime}+m_{13}+m_{23}+m_{33}+m_{44}\right)_{-j_{1}-j_{5}} j_{1} ! j_{2} ! j_{3} ! j_{4} ! j_{5} !\left(m_{22}-m_{13}^{\prime}-j_{2}\right) !\left(m_{33}-m_{33}^{\prime}-j_{4}\right) !} \\
& \times \frac{\left(-m_{11}^{\prime}\right)_{m_{11}+j_{1}+j_{2}}}{\left(m_{13}-m_{22}^{\prime}-j_{1}\right) ! m_{11} ! m_{12} ! m_{23} ! m_{34} !} F_{7} \prod_{1 \leq a \leq 4} \frac{\left(u_{a}^{7}\right)^{m_{a}^{\prime}}}{m_{a}^{\prime} !} \prod_{1 \leq a \leq b \leq 4} \frac{\left(1-v_{a b}^{7}\right)^{m_{a b}^{\prime}}}{m_{a b}^{\prime} !} .
\end{aligned}
$$

At this point, we introduce $m_{44}=m-m_{13}$ and then change $m_{13}$ by $m_{13} \rightarrow m_{13}+m_{22}^{\prime}+j_{1}$. This allows us to sum over $m_{13}$,

$$
\begin{aligned}
& G_{7 R}=\sum \frac{\left(p_{4}-m_{1}^{\prime}\right)_{m_{4}^{\prime}+m_{14}^{\prime}}\left(-h_{6}\right)_{m_{4}^{\prime}+m_{14}^{\prime}+m_{24}^{\prime}+m_{24}+m_{34}^{\prime}-j_{3}}\left(p_{4}-h_{3}+h_{6}\right)_{m_{4}^{\prime}+m_{11}^{\prime}+m_{22}^{\prime}+m_{22}-j_{2}}}{\left(p_{2}+h_{3}+m_{1}^{\prime}\right)_{-m_{4}^{\prime}}\left(p_{4}-h_{3}\right)_{2 m_{4}^{\prime}+m_{11}^{\prime}+m_{14}^{\prime}+m_{22}^{\prime}+m_{22}+m_{24}^{\prime}+m_{24}+m_{34}^{\prime}-j_{2}-j_{3}}\left(p_{4}-h_{3}+1-d / 2\right)_{m_{4}^{\prime}}} \\
& \times \frac{\left(p_{2}+h_{3}\right)_{m_{1}^{\prime}-m_{4}^{\prime}+m_{23}}\left(p_{3}\right)_{-m_{1}^{\prime}+m_{2}^{\prime}+m_{3}^{\prime}+m_{12}+m_{33}}\left(\bar{p}_{3}+h_{2}+h_{5}\right)_{m_{3}^{\prime}+m_{11}+m_{34}}\left(p_{3}-h_{2}+h_{4}\right)_{m_{2}^{\prime}+m_{12}}}{\left(p_{3}-h_{2}\right)_{2 m_{2}^{\prime}+m_{12}+m+m_{23}+m_{33}}\left(p_{3}-h_{2}+1-d / 2\right)_{m_{2}^{\prime}}}
\end{aligned}
$$

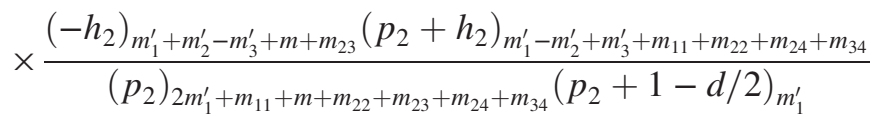

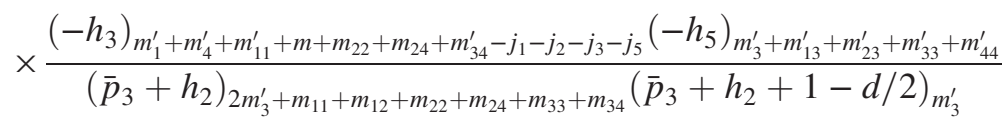

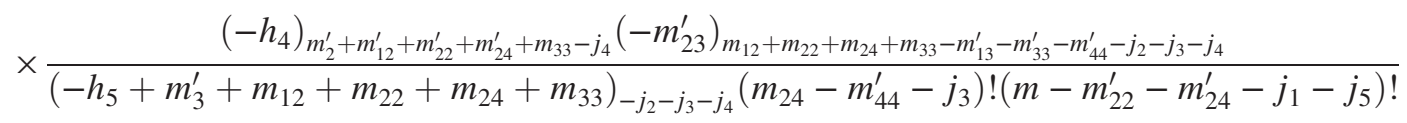

$$
\begin{aligned}
& \times \frac{\left(-m_{34}^{\prime}\right)_{m_{34}+j_{3}+j_{5}}\left(-m_{12}^{\prime}\right)_{m+m_{23}-m_{22}^{\prime}-m_{24}^{\prime}-j_{1}+j_{4}-j_{5}}}{\left(-h_{4}+m_{2}^{\prime}+m+m_{23}+m_{33}\right)_{-j_{1}-j_{5}} j_{1} ! j_{2} ! j_{3} ! j_{4} ! j_{5} !\left(m_{22}-m_{13}^{\prime}-j_{2}\right) !\left(m_{33}-m_{33}^{\prime}-j_{4}\right) !} \\
& \times \frac{\left(-m_{11}^{\prime}\right)_{m_{11}+j_{1}+j_{2}}}{m_{11} ! m_{12} ! m_{23} ! m_{34} !} F_{7} \prod_{1 \leq a \leq 4} \frac{\left(u_{a}^{7}\right)^{m_{a}^{\prime}}}{m_{a}^{\prime} !} \prod_{1 \leq a \leq b \leq 4} \frac{\left(1-v_{a b}^{7}\right)^{m_{a b}^{\prime}}}{m_{a b}^{\prime} !} \text {. }
\end{aligned}
$$

In the same spirit, we define $m_{24}=n-m_{22}$ and change $m_{22}$ by $m_{22} \rightarrow m_{22}+m_{13}^{\prime}+j_{2}$ to evaluate the sum over $m_{22}$, which leads to 


$$
\begin{aligned}
& G_{7 R}=\sum \frac{\left(p_{4}-m_{1}^{\prime}\right)_{m_{4}^{\prime}+m_{14}^{\prime}}\left(-h_{6}\right)_{m_{4}^{\prime}+m_{14}^{\prime}+m_{24}^{\prime}+m_{34}^{\prime}+m_{44}^{\prime}}\left(p_{4}-h_{3}+h_{6}\right)_{m_{4}^{\prime}+m_{11}^{\prime}+m_{13}^{\prime}+m_{22}^{\prime}}}{\left(p_{2}+h_{3}+m_{1}^{\prime}\right)_{-m_{4}^{\prime}}\left(p_{4}-h_{3}\right)_{2 m_{4}^{\prime}+m_{11}^{\prime}+m_{13}^{\prime}+m_{14}^{\prime}+m_{22}^{\prime}+m_{24}^{\prime}+m_{34}^{\prime}+m_{44}^{\prime}}\left(p_{4}-h_{3}+1-d / 2\right)_{m_{4}^{\prime}}} \\
& \times \frac{\left(p_{2}+h_{3}\right)_{m_{1}^{\prime}-m_{4}^{\prime}+m_{23}}\left(p_{3}\right)_{-m_{1}^{\prime}+m_{2}^{\prime}+m_{3}^{\prime}+m_{12}+m_{33}}\left(\bar{p}_{3}+h_{2}+h_{5}\right)_{m_{3}^{\prime}+m_{11}+m_{34}}\left(p_{3}-h_{2}+h_{4}\right)_{m_{2}^{\prime}+m_{12}}}{\left(p_{3}-h_{2}\right)_{2 m_{2}^{\prime}+m_{12}+m+m_{23}+m_{33}}\left(p_{3}-h_{2}+1-d / 2\right)_{m_{2}^{\prime}}} \\
& \times \frac{\left(-h_{2}\right)_{m_{1}^{\prime}+m_{2}^{\prime}-m_{3}^{\prime}+m+m_{23}}\left(p_{2}+h_{2}\right)_{m_{1}^{\prime}-m_{2}^{\prime}+m_{3}^{\prime}+m_{11}+n+m_{34}}}{\left(p_{2}\right)_{2 m_{1}^{\prime}+m_{11}+m+n+m_{23}+m_{34}}\left(p_{2}+1-d / 2\right)_{m_{1}^{\prime}}} \\
& \times \frac{\left(-h_{3}\right)_{m_{1}^{\prime}+m_{4}^{\prime}+m_{11}^{\prime}+m+n+m_{34}^{\prime}-j_{1}-j_{2}-j_{3}-j_{5}}\left(-h_{5}\right)_{m_{3}^{\prime}+m_{13}^{\prime}+m_{23}^{\prime}+m_{33}^{\prime}+m_{44}^{\prime}}\left(-h_{4}\right)_{m_{2}^{\prime}+m_{12}^{\prime}+m_{22}^{\prime}+m_{24}^{\prime}+m_{33}-j_{4}}}{\left(\bar{p}_{3}+h_{2}\right)_{2 m_{3}^{\prime}+m_{11}+m_{12}+n+m_{33}+m_{34}}\left(\bar{p}_{3}+h_{2}+1-d / 2\right)_{m_{3}^{\prime}}} \\
& \times \frac{\left(-m_{23}^{\prime}\right)_{m_{12}+n+m_{33}-m_{13}^{\prime}-m_{33}^{\prime}-m_{44}^{\prime}-j_{2}-j_{3}-j_{4}}}{\left(-h_{5}+m_{3}^{\prime}+m_{12}+n+m_{33}\right)_{-j_{2}-j_{3}-j_{4}}\left(n-m_{13}^{\prime}-m_{44}^{\prime}-j_{2}-j_{3}\right) !\left(m-m_{22}^{\prime}-m_{24}^{\prime}-j_{1}-j_{5}\right) !} \\
& \times \frac{\left(-m_{34}^{\prime}\right)_{m_{34}+j_{3}+j_{5}}\left(-m_{12}^{\prime}\right)_{m+m_{23}-m_{22}^{\prime}-m_{24}^{\prime}-j_{1}+j_{4}-j_{5}}}{\left(-h_{4}+m_{2}^{\prime}+m+m_{23}+m_{33}\right)_{-j_{1}-j_{5}} j_{1} ! j_{2} ! j_{3} ! j_{4} ! j_{5} !\left(m_{33}-m_{33}^{\prime}-j_{4}\right) !} \\
& \times \frac{\left(-m_{11}^{\prime}\right)_{m_{11}+j_{1}+j_{2}}}{m_{11} ! m_{12} ! m_{23} ! m_{34} !} F_{7} \prod_{1 \leq a \leq 4} \frac{\left(u_{a}^{7}\right)^{m_{a}^{\prime}}}{m_{a}^{\prime} !} \prod_{1 \leq a \leq b \leq 4} \frac{\left(1-v_{a b}^{7}\right)^{m_{a b}^{\prime}}}{m_{a b}^{\prime} !} .
\end{aligned}
$$

We now redefine $m_{34}=k_{1}-m_{11}, j_{5}=k_{2}-j_{1}, j_{3}=k_{3}-j_{2}$, and compute the sums over $j_{1}, m_{11}$, and $j_{2}$, to obtain

$$
\begin{aligned}
& G_{7 R}=\sum \frac{\left(p_{4}-m_{1}^{\prime}\right)_{m_{4}^{\prime}+m_{14}^{\prime}}\left(-h_{6}\right)_{m_{4}^{\prime}+m_{14}^{\prime}+m_{24}^{\prime}+m_{34}^{\prime}+m_{44}^{\prime}}\left(p_{4}-h_{3}+h_{6}\right)_{m_{4}^{\prime}+m_{11}^{\prime}+m_{13}^{\prime}+m_{22}^{\prime}}}{\left(p_{2}+h_{3}+m_{1}^{\prime}\right)_{-m_{4}^{\prime}}\left(p_{4}-h_{3}\right)_{2 m_{4}^{\prime}+m_{11}^{\prime}+m_{13}^{\prime}+m_{14}^{\prime}+m_{22}^{\prime}+m_{24}^{\prime}+m_{34}^{\prime}+m_{44}^{\prime}}\left(p_{4}-h_{3}+1-d / 2\right)_{m_{4}^{\prime}}} \\
& \times \frac{\left(p_{2}+h_{3}\right)_{m_{1}^{\prime}-m_{4}^{\prime}+m_{23}}\left(p_{3}\right)_{-m_{1}^{\prime}+m_{2}^{\prime}+m_{3}^{\prime}+m_{12}+m_{33}}\left(\bar{p}_{3}+h_{2}+h_{5}\right)_{m_{3}^{\prime}+k_{1}}\left(p_{3}-h_{2}+h_{4}\right)_{m_{2}^{\prime}+m_{12}}}{\left(p_{3}-h_{2}\right)_{2 m_{2}^{\prime}+m_{12}+m+m_{23}+m_{33}}\left(p_{3}-h_{2}+1-d / 2\right)_{m_{2}^{\prime}}} \\
& \times \frac{\left(-h_{2}\right)_{m_{1}^{\prime}+m_{2}^{\prime}-m_{3}^{\prime}+m+m_{23}}\left(p_{2}+h_{2}\right)_{m_{1}^{\prime}-m_{2}^{\prime}+m_{3}^{\prime}+k_{1}+n}}{\left(p_{2}\right)_{2 m_{1}^{\prime}+k_{1}+m+n+m_{23}}\left(p_{2}+1-d / 2\right)_{m_{1}^{\prime}}} \\
& \times \frac{\left(-h_{3}\right)_{m_{1}^{\prime}+m_{4}^{\prime}+m_{11}^{\prime}+m+n+m_{34}^{\prime}-k_{2}-k_{3}}\left(-h_{5}\right)_{m_{3}^{\prime}+m_{13}^{\prime}+m_{23}^{\prime}+m_{33}^{\prime}+m_{44}^{\prime}}\left(-h_{4}\right)_{m_{2}^{\prime}+m_{12}^{\prime}+m_{22}^{\prime}+m_{24}^{\prime}+m_{33}-j_{4}}}{\left(\bar{p}_{3}+h_{2}\right)_{2 m_{3}^{\prime}+k_{1}+m_{12}+n+m_{33}}\left(\bar{p}_{3}+h_{2}+1-d / 2\right)_{m_{3}^{\prime}}} \\
& \times \frac{\left(-m_{23}^{\prime}\right)_{m_{12}+n+m_{33}-m_{13}^{\prime}-m_{33}^{\prime}-m_{44}^{\prime}-k_{3}-j_{4}}}{\left(-h_{5}+m_{3}^{\prime}+m_{12}+n+m_{33}\right)_{-k_{3}-j_{4}}\left(n-m_{13}^{\prime}-m_{44}^{\prime}-k_{3}\right) !\left(m-m_{22}^{\prime}-m_{24}^{\prime}-k_{2}\right) !} \\
& \times \frac{\left(-m_{12}^{\prime}\right)_{m+m_{23}-m_{22}^{\prime}-m_{24}^{\prime}-k_{2}+j_{4}}}{\left(-h_{4}+m_{2}^{\prime}+m+m_{23}+m_{33}\right)_{-k_{2}} k_{1} ! k_{2} ! k_{3} ! j_{4} !\left(m_{33}-m_{33}^{\prime}-j_{4}\right) !} \\
& \times \frac{\left(-m_{11}^{\prime}-m_{34}^{\prime}\right)_{k_{1}+k_{2}+k_{3}}}{m_{12} ! m_{23} !} F_{7} \prod_{1 \leq a \leq 4} \frac{\left(u_{a}^{7}\right)^{m_{a}^{\prime}}}{m_{a}^{\prime} !} \prod_{1 \leq a \leq b \leq 4} \frac{\left(1-v_{a b}^{7}\right)^{m_{a b}^{\prime}}}{m_{a b}^{\prime} !} .
\end{aligned}
$$


We then shift $m$ by $m \rightarrow m+m_{22}^{\prime}+m_{24}^{\prime}+k_{2}$ and use the first identity in (A.3) to rewrite the sum over $k_{2}$, leading to

$$
\begin{aligned}
& G_{7 R}=\sum \frac{\left(p_{4}-m_{1}^{\prime}\right)_{m_{4}^{\prime}+m_{14}^{\prime}}\left(-h_{6}\right)_{m_{4}^{\prime}+m_{14}^{\prime}+m_{24}^{\prime}+m_{34}^{\prime}+m_{44}^{\prime}}\left(p_{4}-h_{3}+h_{6}\right)_{m_{4}^{\prime}+m_{11}^{\prime}+m_{13}^{\prime}+m_{22}^{\prime}}}{\left(p_{2}+h_{3}+m_{1}^{\prime}\right)_{-m_{4}^{\prime}}\left(p_{4}-h_{3}\right)_{2 m_{4}^{\prime}+m_{11}^{\prime}+m_{13}^{\prime}+m_{14}^{\prime}+m_{22}^{\prime}+m_{24}^{\prime}+m_{34}^{\prime}+m_{44}^{\prime}}\left(p_{4}-h_{3}+1-d / 2\right)_{m_{4}^{\prime}}} \\
& \times \frac{\left(p_{2}+h_{3}\right)_{m_{1}^{\prime}-m_{4}^{\prime}+m_{23}}\left(p_{3}\right)_{-m_{1}^{\prime}+m_{2}^{\prime}+m_{3}^{\prime}+m_{12}+m_{33}}\left(\bar{p}_{3}+h_{2}+h_{5}\right)_{m_{3}^{\prime}+k_{1}}\left(p_{3}-h_{2}+h_{4}\right)_{m_{2}^{\prime}+m_{12}+k_{2}}}{\left(p_{3}-h_{2}\right)_{2 m_{2}^{\prime}+m_{12}+m_{22}^{\prime}+m_{24}^{\prime}+m+m_{23}+m_{33}+k_{2}}\left(p_{3}-h_{2}+1-d / 2\right)_{m_{2}^{\prime}}}
\end{aligned}
$$

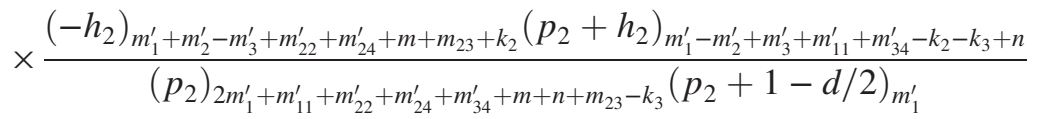

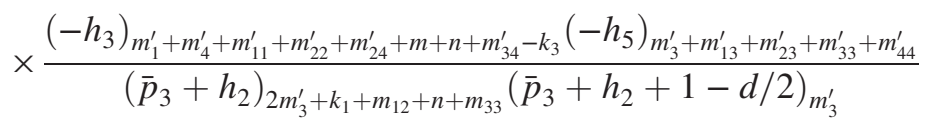

$$
\begin{aligned}
& \times \frac{\left(-h_{4}\right)_{m_{2}^{\prime}+m_{12}^{\prime}+m_{22}^{\prime}+m_{24}^{\prime}+m_{33}-j_{4}}\left(-m_{23}^{\prime}\right)_{m_{12}+n+m_{33}-m_{13}^{\prime}-m_{33}^{\prime}-m_{44}^{\prime}-k_{3}-j_{4}}}{\left(-h_{5}+m_{3}^{\prime}+m_{12}+n+m_{33}\right)_{-k_{3}-j_{4}}\left(n-m_{13}^{\prime}-m_{44}^{\prime}-k_{3}\right) ! m !} \\
& \times \frac{(-1)^{k_{2}}\left(-m_{12}^{\prime}\right)_{m+m_{23}+j_{4}}}{k_{1} ! k_{2} ! k_{3} ! j_{4} !\left(m_{33}-m_{33}^{\prime}-j_{4}\right) !} \frac{\left(-m_{11}^{\prime}-m_{34}^{\prime}\right)_{k_{1}+k_{2}+k_{3}}}{m_{12} ! m_{23} !} F_{7} \prod_{1 \leq a \leq 4} \frac{\left(u_{a}^{7}\right)^{m_{a}^{\prime}}}{m_{a}^{\prime} !} \prod_{1 \leq a \leq b \leq 4} \frac{\left(1-v_{a b}^{7}\right)^{m_{a b}^{\prime}}}{m_{a b}^{\prime} !} .
\end{aligned}
$$

With these modifications, we can complete the sum over $k_{1}$ and obtain

$$
\begin{aligned}
& G_{7 R}=\sum \frac{\left(p_{4}-m_{1}^{\prime}\right)_{m_{4}^{\prime}+m_{14}^{\prime}}\left(-h_{6}\right)_{m_{4}^{\prime}+m_{14}^{\prime}+m_{24}^{\prime}+m_{34}^{\prime}+m_{44}^{\prime}}\left(p_{4}-h_{3}+h_{6}\right)_{m_{4}^{\prime}+m_{11}^{\prime}+m_{13}^{\prime}+m_{22}^{\prime}}}{\left(p_{2}+h_{3}+m_{1}^{\prime}\right)_{-m_{4}^{\prime}}\left(p_{4}-h_{3}\right)_{2 m_{4}^{\prime}+m_{11}^{\prime}+m_{13}^{\prime}+m_{14}^{\prime}+m_{22}^{\prime}+m_{24}^{\prime}+m_{34}^{\prime}+m_{44}^{\prime}}\left(p_{4}-h_{3}+1-d / 2\right)_{m_{4}^{\prime}}} \\
& \times \frac{\left(p_{2}+h_{3}\right)_{m_{1}^{\prime}-m_{4}^{\prime}+m_{23}}\left(p_{3}\right)_{-m_{1}^{\prime}+m_{2}^{\prime}+m_{3}^{\prime}+m_{12}+m_{33}}\left(\bar{p}_{3}+h_{2}+h_{5}\right)_{m_{3}^{\prime}}\left(p_{3}-h_{2}+h_{4}\right)_{m_{2}^{\prime}+m_{12}+k_{2}}}{\left(p_{3}-h_{2}\right)_{2 m_{2}^{\prime}+m_{12}+m_{22}^{\prime}+m_{24}^{\prime}+m+m_{23}+m_{33}+k_{2}}\left(p_{3}-h_{2}+1-d / 2\right)_{m_{2}^{\prime}}} \\
& \times \frac{\left(-h_{2}\right)_{m_{1}^{\prime}+m_{2}^{\prime}-m_{3}^{\prime}+m_{22}^{\prime}+m_{24}^{\prime}+m+m_{23}+k_{2}}\left(p_{2}+h_{2}\right)_{m_{1}^{\prime}-m_{2}^{\prime}+m_{3}^{\prime}+m_{11}^{\prime}+m_{34}^{\prime}-k_{2}-k_{3}+n}}{\left(p_{2}\right)_{2 m_{1}^{\prime}+m_{11}^{\prime}+m_{22}^{\prime}+m_{24}^{\prime}+m_{34}^{\prime}+m+n+m_{23}-k_{3}}\left(p_{2}+1-d / 2\right)_{m_{1}^{\prime}}} \\
& \times \frac{\left(-h_{3}\right)_{m_{1}^{\prime}+m_{4}^{\prime}+m_{11}^{\prime}+m_{22}^{\prime}+m_{24}^{\prime}+m+n+m_{34}^{\prime}-k_{3}}\left(-h_{5}\right)_{m_{3}^{\prime}+m_{13}^{\prime}+m_{23}^{\prime}+m_{33}^{\prime}+m_{44}^{\prime}}\left(-h_{4}\right)_{m_{2}^{\prime}+m_{12}^{\prime}+m_{22}^{\prime}+m_{24}^{\prime}+m_{33}-j_{4}}}{\left(\bar{p}_{3}+h_{2}\right)_{2 m_{3}^{\prime}+m_{11}^{\prime}+m_{34}^{\prime}+m_{12}+n+m_{33}-k_{2}-k_{3}}\left(\bar{p}_{3}+h_{2}+1-d / 2\right)_{m_{3}^{\prime}}} \\
& \times \frac{\left(-h_{5}+m_{3}^{\prime}+m_{12}+n+m_{33}\right)_{m_{11}^{\prime}+m_{34}^{\prime}-k_{2}-k_{3}}\left(-m_{23}^{\prime}\right)_{m_{12}+n+m_{33}-m_{13}^{\prime}-m_{33}^{\prime}-m_{44}^{\prime}-k_{3}-j_{4}}}{\left(-h_{5}+m_{3}^{\prime}+m_{12}+n+m_{33}\right)_{-k_{3}-j_{4}}\left(n-m_{13}^{\prime}-m_{44}^{\prime}-k_{3}\right) ! m !} \\
& \times \frac{(-1)^{k_{2}}\left(-m_{12}^{\prime}\right)_{m+m_{23}+j_{4}}}{k_{2} ! k_{3} ! j_{4} !\left(m_{33}-m_{33}^{\prime}-j_{4}\right) !} \frac{\left(-m_{11}^{\prime}-m_{34}^{\prime}\right)_{k_{2}+k_{3}}}{m_{12} ! m_{23} !} F_{7} \prod_{1 \leq a \leq 4} \frac{\left(u_{a}^{7}\right)^{m_{a}^{\prime}}}{m_{a}^{\prime} !} \prod_{1 \leq a \leq b \leq 4} \frac{\left(1-v_{a b}^{7}\right)^{m_{a b}^{\prime}}}{m_{a b}^{\prime} !} .
\end{aligned}
$$


It is then possible to shift $n$ by $n \rightarrow n+m_{13}^{\prime}+m_{44}^{\prime}+k_{3}$ and evaluate the sum over $k_{3},{ }^{6}$ leading to

$$
\begin{aligned}
& G_{7 R}=\sum \frac{\left(p_{4}-m_{1}^{\prime}\right)_{m_{4}^{\prime}+m_{14}^{\prime}}\left(-h_{6}\right)_{m_{4}^{\prime}+m_{14}^{\prime}+m_{24}^{\prime}+m_{34}^{\prime}+m_{44}^{\prime}}\left(p_{4}-h_{3}+h_{6}\right)_{m_{4}^{\prime}+m_{11}^{\prime}+m_{13}^{\prime}+m_{22}^{\prime}}}{\left(p_{2}+h_{3}+m_{1}^{\prime}\right)_{-m_{4}^{\prime}}\left(p_{4}-h_{3}\right)_{2 m_{4}^{\prime}+m_{11}^{\prime}+m_{13}^{\prime}+m_{14}^{\prime}+m_{22}^{\prime}+m_{24}^{\prime}+m_{34}^{\prime}+m_{44}^{\prime}}\left(p_{4}-h_{3}+1-d / 2\right)_{m_{4}^{\prime}}}
\end{aligned}
$$

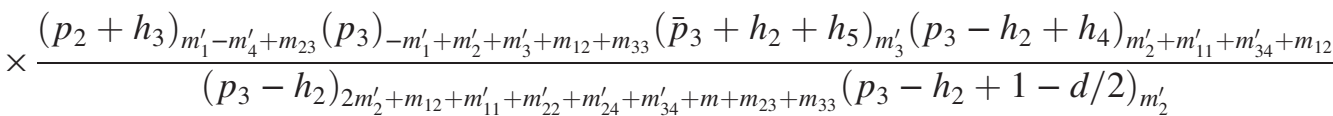

$$
\begin{aligned}
& \times \frac{\left(-h_{2}\right)_{m_{1}^{\prime}+m_{2}^{\prime}-m_{3}^{\prime}+m_{11}^{\prime}+m_{22}^{\prime}+m_{24}^{\prime}+m_{34}^{\prime}+m+m_{23}}\left(p_{2}+h_{2}\right)_{m_{1}^{\prime}-m_{2}^{\prime}+m_{3}^{\prime}+m_{13}^{\prime}+m_{44}^{\prime}+n}}{\left(p_{2}\right)_{2 m_{1}^{\prime}+m_{11}^{\prime}+m_{13}^{\prime}+m_{22}^{\prime}+m_{24}^{\prime}+m_{34}^{\prime}+m_{44}^{\prime}+m+n+m_{23}}\left(p_{2}+1-d / 2\right)_{m_{1}^{\prime}}} \\
& \times \frac{\left(-h_{3}\right)_{m_{1}^{\prime}+m_{4}^{\prime}+m_{11}^{\prime}+m_{13}^{\prime}+m_{22}^{\prime}+m_{24}^{\prime}+m_{44}^{\prime}+m+n+m_{34}^{\prime}}\left(-h_{5}\right)_{m_{3}^{\prime}+m_{13}^{\prime}+m_{23}^{\prime}+m_{33}^{\prime}+m_{44}^{\prime}}}{\left.\left(\bar{p}_{3}+h_{2}\right)_{2 m_{3}^{\prime}+m_{13}^{\prime}+m_{44}^{\prime}+m_{12}+n+m_{33}} \bar{p}_{3}+h_{2}+1-d / 2\right)_{m_{3}^{\prime}}} \\
& \times \frac{\left(-h_{4}\right)_{m_{2}^{\prime}+m_{12}^{\prime}+m_{22}^{\prime}+m_{24}^{\prime}+m_{33}-j_{4}}\left(-m_{23}^{\prime}\right)_{m_{12}+n+m_{33}-m_{33}^{\prime}-j_{4}}}{\left(-h_{5}+m_{3}^{\prime}+m_{13}^{\prime}+m_{44}^{\prime}+m_{12}+n+m_{33}\right)_{-j_{4}} n ! m !} \\
& \times \frac{\left(-m_{12}^{\prime}\right)_{m+m_{23}+j_{4}}}{j_{4} !\left(m_{33}-m_{33}^{\prime}-j_{4}\right) ! m_{12} ! m_{23} !} F_{7} \prod_{1 \leq a \leq 4} \frac{\left(u_{a}^{7}\right)^{m_{a}^{\prime}}}{m_{a}^{\prime} !} \prod_{1 \leq a \leq b \leq 4} \frac{\left(1-v_{a b}^{7}\right)^{m_{a b}^{\prime}}}{m_{a b}^{\prime} !} \text {. }
\end{aligned}
$$

As before, we shift $m_{33}$ by $m_{33} \rightarrow m_{33}+m_{33}^{\prime}+j_{4}$ and rewrite the sum over $m_{23}$ with the help of the first identity in (A.3). This allows us to express $G_{7 R}$ as

$$
\begin{aligned}
& G_{7 R}=\sum \frac{\left(p_{4}-m_{1}^{\prime}\right)_{m_{4}^{\prime}+m_{14}^{\prime}}\left(-h_{6}\right)_{m_{4}^{\prime}+m_{14}^{\prime}+m_{24}^{\prime}+m_{34}^{\prime}+m_{44}^{\prime}}\left(p_{4}-h_{3}+h_{6}\right)_{m_{4}^{\prime}+m_{11}^{\prime}+m_{13}^{\prime}+m_{22}^{\prime}}}{\left(p_{2}+h_{3}+m_{1}^{\prime}\right)_{-m_{4}^{\prime}}\left(p_{4}-h_{3}\right)_{2 m_{4}^{\prime}+m_{11}^{\prime}+m_{13}^{\prime}+m_{14}^{\prime}+m_{22}^{\prime}+m_{24}^{\prime}+m_{34}^{\prime}+m_{44}^{\prime}}\left(p_{4}-h_{3}+1-d / 2\right)_{m_{4}^{\prime}}}
\end{aligned}
$$

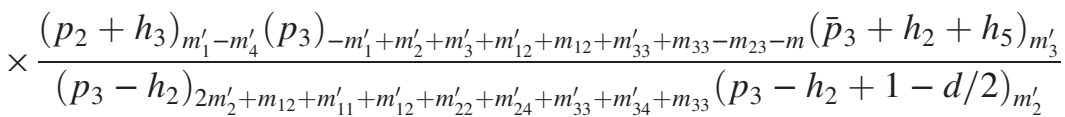

$$
\begin{aligned}
& \times \frac{\left(-h_{2}\right)_{m_{1}^{\prime}+m_{2}^{\prime}-m_{3}^{\prime}+m_{11}^{\prime}+m_{22}^{\prime}+m_{24}^{\prime}+m_{34}^{\prime}+m+m_{23}}\left(p_{2}+h_{2}\right)_{m_{1}^{\prime}-m_{2}^{\prime}+m_{3}^{\prime}+m_{13}^{\prime}+m_{44}^{\prime}+n}}{\left(p_{2}\right)_{2 m_{1}^{\prime}+m_{11}^{\prime}+m_{13}^{\prime}+m_{22}^{\prime}+m_{24}^{\prime}+m_{34}^{\prime}+m_{44}^{\prime}+m+n+m_{23}}\left(p_{2}+1-d / 2\right)_{m_{1}^{\prime}}} \\
& \times \frac{\left(-h_{3}\right)_{m_{1}^{\prime}+m_{4}^{\prime}+m_{11}^{\prime}+m_{13}^{\prime}+m_{22}^{\prime}+m_{24}^{\prime}+m_{44}^{\prime}+m_{34}^{\prime}+m_{23}+m+n}\left(-h_{5}\right)_{m_{3}^{\prime}+m_{13}^{\prime}+m_{23}^{\prime}+m_{33}^{\prime}+m_{44}^{\prime}}}{\left(\bar{p}_{3}+h_{2}\right)_{2 m_{3}^{\prime}+m_{13}^{\prime}+m_{33}^{\prime}+m_{44}^{\prime}+m_{12}+n+m_{33}+j_{4}}\left(\bar{p}_{3}+h_{2}+1-d / 2\right)_{m_{3}^{\prime}}} \\
& \times \frac{\left(p_{3}-h_{2}+h_{4}\right)_{m_{2}^{\prime}+m_{11}^{\prime}+m_{34}^{\prime}+m_{12}}\left(-h_{4}\right)_{m_{2}^{\prime}+m_{12}^{\prime}+m_{22}^{\prime}+m_{24}^{\prime}+m_{33}^{\prime}+m_{33}}\left(-m_{23}^{\prime}\right)_{m_{12}+n+m_{33}}}{n ! m !} \\
& \times \frac{(-1)^{m_{23}}\left(-h_{5}+m_{3}^{\prime}+m_{13}^{\prime}+m_{33}^{\prime}+m_{44}^{\prime}+m_{12}+n+m_{33}\right)_{j_{4}}\left(-m_{12}^{\prime}\right)_{m+m_{23}+j_{4}}}{j_{4} ! m_{33} ! m_{12} ! m_{23} !} \\
& \times F_{7} \prod_{1 \leq a \leq 4} \frac{\left(u_{a}^{7}\right)^{m_{a}^{\prime}}}{m_{a}^{\prime} !} \prod_{1 \leq a \leq b \leq 4} \frac{\left(1-v_{a b}^{7}\right)^{m_{a b}^{\prime}}}{m_{a b}^{\prime} !} .
\end{aligned}
$$

\footnotetext{
${ }^{6}$ Using the binomial identity as in (A.1) with $v=1$, the sum over $k_{3}$ forces $k_{2}=m_{11}^{\prime}+m_{34}^{\prime}$.
} 
At this point, it is trivial to see that the sum over $j_{4}$ gives

$$
\begin{aligned}
& G_{7 R}=\sum \frac{\left(p_{4}-m_{1}^{\prime}\right)_{m_{4}^{\prime}+m_{14}^{\prime}}\left(-h_{6}\right)_{m_{4}^{\prime}+m_{14}^{\prime}+m_{24}^{\prime}+m_{34}^{\prime}+m_{44}^{\prime}}\left(p_{4}-h_{3}+h_{6}\right)_{m_{4}^{\prime}+m_{11}^{\prime}+m_{13}^{\prime}+m_{22}^{\prime}}}{\left(p_{2}+h_{3}+m_{1}^{\prime}\right)_{-m_{4}^{\prime}}\left(p_{4}-h_{3}\right)_{2 m_{4}^{\prime}+m_{11}^{\prime}+m_{13}^{\prime}+m_{14}^{\prime}+m_{22}^{\prime}+m_{24}^{\prime}+m_{34}^{\prime}+m_{44}^{\prime}}\left(p_{4}-h_{3}+1-d / 2\right)_{m_{4}^{\prime}}}
\end{aligned}
$$

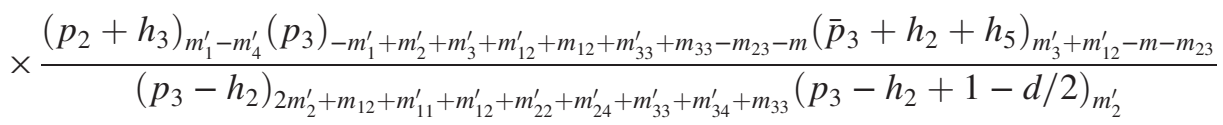

$$
\begin{aligned}
& \times \frac{\left(-h_{2}\right)_{m_{1}^{\prime}+m_{2}^{\prime}-m_{3}^{\prime}+m_{11}^{\prime}+m_{22}^{\prime}+m_{24}^{\prime}+m_{34}^{\prime}+m+m_{23}}\left(p_{2}+h_{2}\right)_{m_{1}^{\prime}-m_{2}^{\prime}+m_{3}^{\prime}+m_{13}^{\prime}+m_{44}^{\prime}+n}}{\left(p_{2}\right)_{2 m_{1}^{\prime}+m_{11}^{\prime}+m_{13}^{\prime}+m_{22}^{\prime}+m_{24}^{\prime}+m_{34}^{\prime}+m_{44}^{\prime}+m+n+m_{23}}\left(p_{2}+1-d / 2\right)_{m_{1}^{\prime}}} \\
& \times \frac{\left(-h_{3}\right)_{m_{1}^{\prime}+m_{4}^{\prime}+m_{11}^{\prime}+m_{13}^{\prime}+m_{22}^{\prime}+m_{24}^{\prime}+m_{44}^{\prime}+m_{34}^{\prime}+m_{23}+m+n}\left(-h_{5}\right)_{m_{3}^{\prime}+m_{13}^{\prime}+m_{23}^{\prime}+m_{33}^{\prime}+m_{44}^{\prime}}}{\left(\bar{p}_{3}+h_{2}\right)_{2 m_{3}^{\prime}+m_{12}^{\prime}+m_{13}^{\prime}+m_{33}^{\prime}+m_{44}^{\prime}+m_{12}+n+m_{33}-m_{23}-m}\left(\bar{p}_{3}+h_{2}+1-d / 2\right)_{m_{3}^{\prime}}} \\
& \times \frac{\left(p_{3}-h_{2}+h_{4}\right)_{m_{2}^{\prime}+m_{11}^{\prime}+m_{34}^{\prime}+m_{12}}\left(-h_{4}\right)_{m_{2}^{\prime}+m_{12}^{\prime}+m_{22}^{\prime}+m_{24}^{\prime}+m_{33}^{\prime}+m_{33}}\left(-m_{23}^{\prime}\right)_{m_{12}+n+m_{33}}}{n ! m !} \\
& \times \frac{(-1)^{m_{23}}\left(-m_{12}^{\prime}\right)_{m+m_{23}}}{m_{33} ! m_{12} ! m_{23} !} F_{7} \frac{\left(u_{a}^{7}\right)^{m_{a}^{\prime}}}{m_{a}^{\prime} !} \prod_{1 \leq a \leq b \leq 4} \frac{\left(1-v_{a b}^{7}\right)^{m_{a b}^{\prime}}}{m_{a b}^{\prime} !} .
\end{aligned}
$$

After redefining $m_{33}=k-m_{12}$, we can compute the sums over $m_{12}, k$, and $n$, leading to

$$
\begin{aligned}
& G_{7 R}=\sum \frac{\left(p_{4}-m_{1}^{\prime}\right)_{m_{4}^{\prime}+m_{14}^{\prime}}\left(-h_{6}\right)_{m_{4}^{\prime}+m_{14}^{\prime}+m_{24}^{\prime}+m_{34}^{\prime}+m_{44}^{\prime}}\left(p_{4}-h_{3}+h_{6}\right)_{m_{4}^{\prime}+m_{11}^{\prime}+m_{13}^{\prime}+m_{22}^{\prime}}}{\left(p_{2}+h_{3}+m_{1}^{\prime}\right)_{-m_{4}^{\prime}}\left(p_{4}-h_{3}\right)_{2 m_{4}^{\prime}+m_{11}^{\prime}+m_{13}^{\prime}+m_{14}^{\prime}+m_{22}^{\prime}+m_{24}^{\prime}+m_{34}^{\prime}+m_{44}^{\prime}}\left(p_{4}-h_{3}+1-d / 2\right)_{m_{4}^{\prime}}}
\end{aligned}
$$

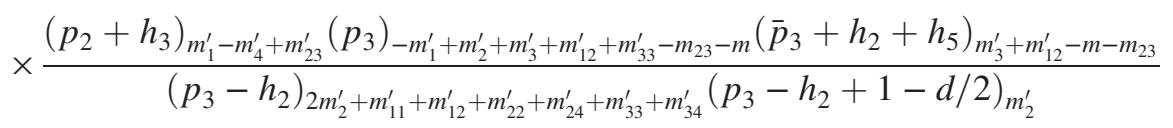

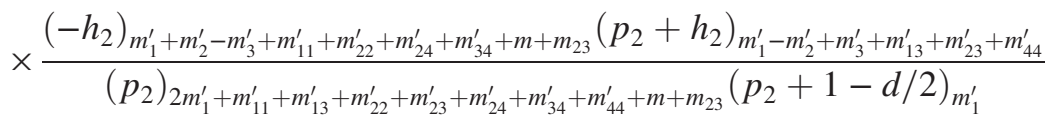

$$
\begin{aligned}
& \times \frac{\left(-h_{3}\right)_{m_{1}^{\prime}+m_{4}^{\prime}+m_{11}^{\prime}+m_{13}^{\prime}+m_{22}^{\prime}+m_{24}^{\prime}+m_{44}^{\prime}+m_{34}^{\prime}+m_{23}+m}\left(-h_{5}\right)_{m_{3}^{\prime}+m_{13}^{\prime}+m_{23}^{\prime}+m_{33}^{\prime}+m_{44}^{\prime}}}{\left(\bar{p}_{3}+h_{2}\right)_{2 m_{3}^{\prime}+m_{12}^{\prime}+m_{13}^{\prime}+m_{23}^{\prime}+m_{33}^{\prime}+m_{44}^{\prime}-m_{23}-m}\left(\bar{p}_{3}+h_{2}+1-d / 2\right)_{m_{3}^{\prime}}} \\
& \times \frac{\left(p_{3}-h_{2}+h_{4}\right)_{m_{2}^{\prime}+m_{11}^{\prime}+m_{34}^{\prime}}\left(-h_{4}\right)_{m_{2}^{\prime}+m_{12}^{\prime}+m_{22}^{\prime}+m_{24}^{\prime}+m_{33}^{\prime}}}{m !} \\
& \times \frac{(-1)^{m_{23}}\left(-m_{12}^{\prime}\right)_{m+m_{23}}}{m_{23} !} F_{7} \prod_{1 \leq a \leq 4} \frac{\left(u_{a}^{7}\right)^{m_{a}^{\prime}}}{m_{a}^{\prime} !} \prod_{1 \leq a \leq b \leq 4} \frac{\left(1-v_{a b}^{7}\right)^{m_{a b}^{\prime}}}{m_{a b}^{\prime} !} .
\end{aligned}
$$


Finally, we introduce $m_{23}=r-m$ and evaluate the sums over $m$ and $r{ }^{7}$ such that

$$
\begin{aligned}
& G_{7 R}=\sum \frac{\left(p_{4}-m_{1}^{\prime}\right)_{m_{4}^{\prime}+m_{14}^{\prime}}\left(-h_{6}\right)_{m_{4}^{\prime}+m_{14}^{\prime}+m_{24}^{\prime}+m_{34}^{\prime}+m_{44}^{\prime}}\left(p_{4}-h_{3}+h_{6}\right)_{m_{4}^{\prime}+m_{11}^{\prime}+m_{13}^{\prime}+m_{22}^{\prime}}}{\left(p_{2}+h_{3}+m_{1}^{\prime}\right)_{-m_{4}^{\prime}}\left(p_{4}-h_{3}\right)_{2 m_{4}^{\prime}+m_{11}^{\prime}+m_{13}^{\prime}+m_{14}^{\prime}+m_{22}^{\prime}+m_{24}^{\prime}+m_{34}^{\prime}+m_{44}^{\prime}}\left(p_{4}-h_{3}+1-d / 2\right)_{m_{4}^{\prime}}} \\
& \times \frac{\left(p_{2}+h_{3}\right)_{m_{1}^{\prime}-m_{4}^{\prime}+m_{23}^{\prime}}\left(p_{3}\right)_{-m_{1}^{\prime}+m_{2}^{\prime}+m_{3}^{\prime}+m_{12}^{\prime}+m_{33}^{\prime}}\left(p_{3}-h_{2}+h_{4}\right)_{m_{2}^{\prime}+m_{11}^{\prime}+m_{34}^{\prime}}\left(\bar{p}_{3}+h_{2}+h_{5}\right)_{m_{3}^{\prime}+m_{12}^{\prime}}}{\left(p_{3}-h_{2}\right)_{2 m_{2}^{\prime}+m_{11}^{\prime}+m_{12}^{\prime}+m_{22}^{\prime}+m_{24}^{\prime}+m_{33}^{\prime}+m_{34}^{\prime}}\left(p_{3}-h_{2}+1-d / 2\right)_{m_{2}^{\prime}}}
\end{aligned}
$$

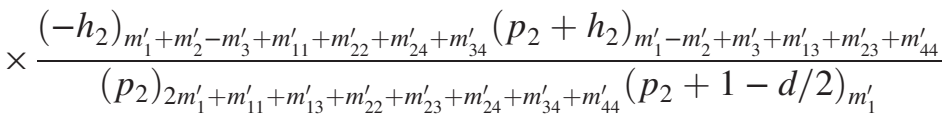

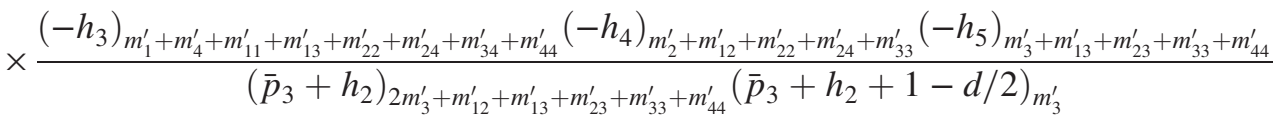

$$
\begin{aligned}
& \times F_{7} \prod_{1 \leq a \leq 4} \frac{\left(u_{a}^{7}\right)^{m_{a}^{\prime}}}{m_{a}^{\prime} !} \prod_{1 \leq a \leq b \leq 4} \frac{\left(1-v_{a b}^{7}\right)^{m_{a b}^{\prime}}}{m_{a b}^{\prime} !} \\
& =G_{7} \text {. }
\end{aligned}
$$

This ends our proof of the invariance of the scalar seven-point correlation functions under reflections (3.1).

\section{Dendrite permutations of the first kind}

Invariance under dendrite permutations of the first kind implies the identity (3.2), which we rewrite as $G_{7}=G_{7 P_{1}}$ to simplify the notation. Once again, $F_{7}$ is invariant under the generator, as can be seen from (2.18). Expressing $G_{7 P_{1}}$ in terms of the original conformal cross-ratios and expanding, we find

$$
\begin{aligned}
& G_{7 P_{1}}=\sum \frac{\left(p_{4}-m_{1}\right)_{m_{4}+m_{14}}\left(-h_{6}\right)_{m_{4}+m_{14}+m_{24}+m_{34}+m_{44}}\left(p_{4}-h_{3}+h_{6}\right)_{m_{4}+m_{11}+m_{13}+m_{22}}}{\left(p_{4}-h_{3}\right)_{2 m_{4}+m_{11}+m_{13}+m_{14}+m_{22}+m_{24}+m_{34}+m_{44}}\left(p_{4}-h_{3}+1-d / 2\right)_{m_{4}}} \\
& \times \frac{\left(p_{2}+h_{3}\right)_{m_{1}-m_{4}+m_{23}}\left(-h_{4}\right)_{m_{2}+m_{11}+m_{34}}\left(\bar{p}_{3}+h_{2}+h_{5}\right)_{m_{3}+m_{12}}}{\left(p_{2}+h_{3}+m_{1}\right)_{-m_{4}}\left(\bar{p}_{3}+h_{2}\right)_{2 m_{3}+m_{13}+m_{44}+m_{12}+m_{23}+m_{33}\left(\bar{p}_{3}+h_{2}+1-d / 2\right)_{m_{3}}}} \\
& \times \frac{\left(p_{3}\right)_{-m_{1}+m_{2}+m_{3}+m_{12}+m_{33}}\left(p_{2}+h_{2}\right)_{m_{1}-m_{2}+m_{3}+m_{13}+m_{44}+m_{23}}\left(-h_{2}\right)_{m_{1}+m_{2}-m_{3}+m_{11}+m_{22}+m_{24}+m_{34}}}{\left(p_{2}\right)_{2 m_{1}+m_{11}+m_{13}+m_{22}+m_{23}+m_{24}+m_{34}+m_{44}}\left(p_{2}+1-d / 2\right)_{m_{1}}} \\
& \times \frac{\left(-h_{3}\right)_{m_{1}+m_{4}+m_{11}+m_{13}+m_{22}+m_{24}+m_{34}+m_{44}}\left(p_{3}-h_{2}+h_{4}\right)_{m_{2}+m_{12}+m_{22}+m_{24}+m_{33}}\left(-h_{5}\right)_{m_{3}+m_{13}+m_{23}+m_{33}+m_{44}}}{\left(p_{3}-h_{2}\right)_{2 m_{2}+m_{11}+m_{12}+m_{22}+m_{24}+m_{33}+m_{34}}\left(p_{3}-h_{2}+1-d / 2\right)_{m_{2}}} \\
& \times\left(\begin{array}{c}
m_{12} \\
k_{12}
\end{array}\right)\left(\begin{array}{l}
m_{13} \\
k_{13}
\end{array}\right)\left(\begin{array}{l}
m_{23} \\
k_{23}
\end{array}\right)\left(\begin{array}{c}
m_{33} \\
k_{33}
\end{array}\right)\left(\begin{array}{c}
m_{44} \\
k_{44}
\end{array}\right)\left(\begin{array}{c}
-p_{3}-h_{5}+m_{1}-m_{2}-k_{12}+k_{13}+k_{23}+k_{44} \\
m_{12}^{\prime}
\end{array}\right) \\
& \times\left(\begin{array}{c}
k_{13} \\
m_{13}^{\prime}
\end{array}\right)\left(\begin{array}{c}
k_{23} \\
m_{23}^{\prime}
\end{array}\right)\left(\begin{array}{c}
h_{5}-m_{3}-k_{13}-k_{23}-k_{33}-k_{44} \\
m_{33}^{\prime}
\end{array}\right)\left(\begin{array}{c}
k_{44} \\
m_{44}^{\prime}
\end{array}\right) \\
& \times(-1)^{k_{12}+k_{13}+k_{23}+k_{33}+k_{44}+m_{12}^{\prime}+m_{13}^{\prime}+m_{23}^{\prime}+m_{33}^{\prime}+m_{44}^{\prime}} F_{7} \prod_{1 \leq a \leq 4} \frac{\left(u_{a}^{7}\right)^{m_{a}}}{m_{a} !} \prod_{1 \leq a \leq b \leq 4} \frac{\left(1-v_{a b}^{7}\right)^{m_{a b}}}{m_{a b} !},
\end{aligned}
$$

and we must now resum the extra sums to rewrite the result as $G_{7}{ }^{8}$

\footnotetext{
${ }^{7}$ Again, the binomial identity (A.1) with $v=1$ implies that the sum over $m$ forces $r=0$.

${ }^{8}$ For simplicity, we wrote the product over the $v_{a b}$ cross-ratios although some of the cross-ratios are mixed as per (3.2). They will be unmixed at the end of the proof.
} 
We first evaluate the sums over $k_{12}$ and $k_{33}$, leading to

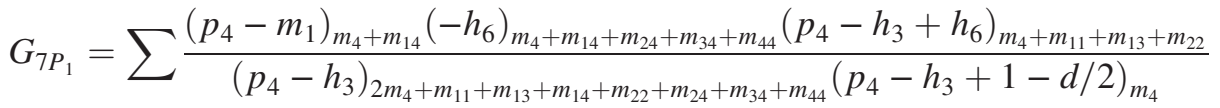

$$
\begin{aligned}
& \times \frac{\left(p_{2}+h_{3}\right)_{m_{1}-m_{4}+m_{23}}\left(-h_{4}\right)_{m_{2}+m_{11}+m_{34}}\left(\bar{p}_{3}+h_{2}+h_{5}\right)_{m_{3}+m_{12}}}{\left(p_{2}+h_{3}+m_{1}\right)_{-m_{4}}\left(\bar{p}_{3}+h_{2}\right)_{2 m_{3}+m_{13}+m_{44}+m_{12}+m_{23}+m_{33}\left(\bar{p}_{3}+h_{2}+1-d / 2\right)_{m_{3}}}}
\end{aligned}
$$

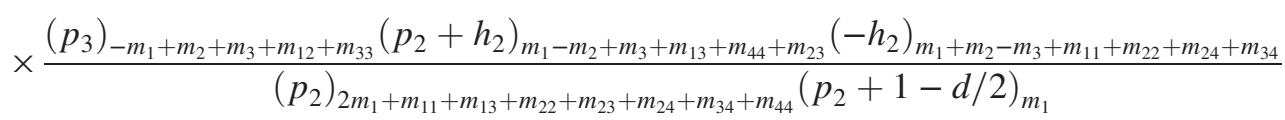

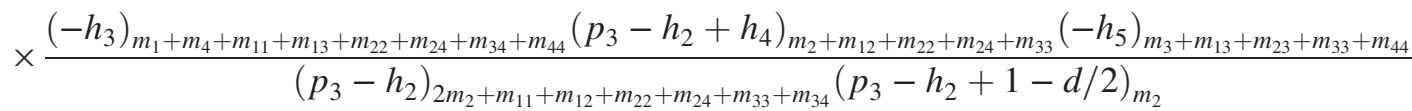

$$
\begin{aligned}
& \times\left(\begin{array}{c}
m_{13} \\
k_{13}
\end{array}\right)\left(\begin{array}{l}
m_{23} \\
k_{23}
\end{array}\right)\left(\begin{array}{c}
m_{44} \\
k_{44}
\end{array}\right) \frac{\left(-m_{12}^{\prime}\right)_{m_{12}}\left(p_{3}+h_{5}-m_{1}+m_{2}+m_{12}-k_{13}-k_{23}-k_{44}\right)_{m_{12}^{\prime}-m_{12}}}{m_{12}^{\prime} !} \\
& \times\left(\begin{array}{c}
k_{13} \\
m_{13}^{\prime}
\end{array}\right)\left(\begin{array}{c}
k_{23} \\
m_{23}^{\prime}
\end{array}\right)\left(\begin{array}{c}
k_{44} \\
m_{44}^{\prime}
\end{array}\right) \frac{\left(-m_{33}^{\prime}\right)_{m_{33}}\left(-h_{5}+m_{3}+m_{33}+k_{13}+k_{23}+k_{44}\right)_{m_{33}^{\prime}-m_{33}}}{m_{33}^{\prime} !} \\
& \times(-1)^{k_{13}+k_{23}+k_{44}+m_{13}^{\prime}+m_{23}^{\prime}+m_{44}^{\prime}} F_{7} \prod_{1 \leq a \leq 4} \frac{\left(u_{a}^{7}\right)^{m_{a}}}{m_{a} !} \prod_{1 \leq a \leq b \leq 4} \frac{\left(1-v_{a b}^{7}\right)^{m_{a b}}}{m_{a b} !} .
\end{aligned}
$$

Using the following identity:

$$
\begin{aligned}
& \left(p_{3}+h_{5}-m_{1}+m_{2}+m_{12}-k_{13}-k_{23}-k_{44}\right)_{m_{12}^{\prime}-m_{12}} \\
& \quad=\sum_{j_{1} \geq 0}\left(\begin{array}{c}
m_{12}^{\prime}-m_{12} \\
j_{1}
\end{array}\right)\left(p_{3}+h_{5}-m_{1}+m_{2}+m_{12}-m_{13}^{\prime}-k_{23}-k_{44}\right)_{m_{12}^{\prime}-m_{12}-j_{1}}\left(-k_{13}+m_{13}^{\prime}\right)_{j_{1}},
\end{aligned}
$$

and then changing the variable such that $k_{13} \rightarrow k_{13}+m_{13}^{\prime}+j_{1}$, we can then evaluate the sum over $k_{13}$ to obtain

$$
\begin{aligned}
& G_{7 P_{1}}=\sum \frac{\left(p_{4}-m_{1}\right)_{m_{4}+m_{14}}\left(-h_{6}\right)_{m_{4}+m_{14}+m_{24}+m_{34}+m_{44}}\left(p_{4}-h_{3}+h_{6}\right)_{m_{4}+m_{11}+m_{13}+m_{22}}}{\left(p_{4}-h_{3}\right)_{2 m_{4}+m_{11}+m_{13}+m_{14}+m_{22}+m_{24}+m_{34}+m_{44}}\left(p_{4}-h_{3}+1-d / 2\right)_{m_{4}}} \\
& \times \frac{\left(p_{2}+h_{3}\right)_{m_{1}-m_{4}+m_{23}}\left(-h_{4}\right)_{m_{2}+m_{11}+m_{34}}\left(\bar{p}_{3}+h_{2}+h_{5}\right)_{m_{3}+m_{12}}}{\left(p_{2}+h_{3}+m_{1}\right)_{-m_{4}}\left(\bar{p}_{3}+h_{2}\right)_{2 m_{3}+m_{13}+m_{44}+m_{12}+m_{23}+m_{33}\left(\bar{p}_{3}+h_{2}+1-d / 2\right)_{m_{3}}}} \\
& \times \frac{\left(p_{3}\right)_{-m_{1}+m_{2}+m_{3}+m_{12}+m_{33}}\left(p_{2}+h_{2}\right)_{m_{1}-m_{2}+m_{3}+m_{13}+m_{44}+m_{23}}\left(-h_{2}\right)_{m_{1}+m_{2}-m_{3}+m_{11}+m_{22}+m_{24}+m_{34}}}{\left(p_{2}\right)_{2 m_{1}+m_{11}+m_{13}+m_{22}+m_{23}+m_{24}+m_{34}+m_{44}}\left(p_{2}+1-d / 2\right)_{m_{1}}}
\end{aligned}
$$

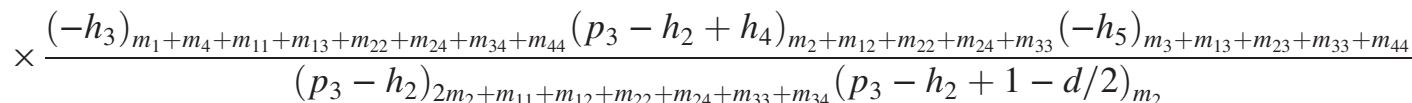

$$
\begin{aligned}
& \times\left(\begin{array}{c}
m_{23} \\
k_{23}
\end{array}\right)\left(\begin{array}{c}
m_{44} \\
k_{44}
\end{array}\right) \frac{m_{13} !\left(-m_{12}^{\prime}\right)_{j_{1}+m_{12}}\left(p_{3}+h_{5}-m_{1}+m_{2}+m_{12}-m_{13}^{\prime}-k_{23}-k_{44}\right)_{m_{12}^{\prime}-m_{12}-j_{1}}}{m_{12}^{\prime} ! m_{13}^{\prime} !\left(m_{13}-m_{13}^{\prime}-j_{1}\right) !} \\
& \times\left(\begin{array}{c}
k_{23} \\
m_{23}^{\prime}
\end{array}\right)\left(\begin{array}{c}
k_{44} \\
m_{44}^{\prime}
\end{array}\right) \frac{\left(-m_{33}^{\prime}\right)_{m_{13}+m_{33}-m_{13}^{\prime}-j_{1}}\left(-h_{5}+m_{3}+m_{13}+m_{33}+k_{23}+k_{44}\right)_{j_{1}+m_{13}^{\prime}+m_{33}^{\prime}-m_{13}-m_{33}}}{j_{1} ! m_{33}^{\prime} !} \\
& \times(-1)^{j_{1}+k_{23}+k_{44}+m_{23}^{\prime}+m_{44}^{\prime}} F_{7} \prod_{1 \leq a \leq 4} \frac{\left(u_{a}^{7}\right)^{m_{a}}}{m_{a} !} \prod_{1 \leq a \leq b \leq 4} \frac{\left(1-v_{a b}^{7}\right)^{m_{a b}}}{m_{a b} !} .
\end{aligned}
$$


In a similar way, we compute the sums over $k_{23}$ and $k_{44}$, giving us

$$
\begin{aligned}
& G_{7 P_{1}}=\sum \frac{\left(p_{4}-m_{1}\right)_{m_{4}+m_{14}}\left(-h_{6}\right)_{m_{4}+m_{14}+m_{24}+m_{34}+m_{44}}\left(p_{4}-h_{3}+h_{6}\right)_{m_{4}+m_{11}+m_{13}+m_{22}}}{\left(p_{4}-h_{3}\right)_{2 m_{4}+m_{11}+m_{13}+m_{14}+m_{22}+m_{24}+m_{34}+m_{44}}\left(p_{4}-h_{3}+1-d / 2\right)_{m_{4}}} \\
& \times \frac{\left(p_{2}+h_{3}\right)_{m_{1}-m_{4}+m_{23}}\left(-h_{4}\right)_{m_{2}+m_{11}+m_{34}}\left(\bar{p}_{3}+h_{2}+h_{5}\right)_{m_{3}+m_{12}}}{\left(p_{2}+h_{3}+m_{1}\right)_{-m_{4}}\left(\bar{p}_{3}+h_{2}\right)_{2 m_{3}+m_{13}+m_{44}+m_{12}+m_{23}+m_{33}\left(\bar{p}_{3}+h_{2}+1-d / 2\right)_{m_{3}}}} \\
& \times \frac{\left(p_{3}\right)_{-m_{1}+m_{2}+m_{3}+m_{12}+m_{33}}\left(p_{2}+h_{2}\right)_{m_{1}-m_{2}+m_{3}+m_{13}+m_{44}+m_{23}}\left(-h_{2}\right)_{m_{1}+m_{2}-m_{3}+m_{11}+m_{22}+m_{24}+m_{34}}}{\left(p_{2}\right)_{2 m_{1}+m_{11}+m_{13}+m_{22}+m_{23}+m_{24}+m_{34}+m_{44}}\left(p_{2}+1-d / 2\right)_{m_{1}}} \\
& \times \frac{\left(-h_{3}\right)_{m_{1}+m_{4}+m_{11}+m_{13}+m_{22}+m_{24}+m_{34}+m_{44}}\left(p_{3}-h_{2}+h_{4}\right)_{m_{2}+m_{12}+m_{22}+m_{24}+m_{33}}}{\left(p_{3}-h_{2}\right)_{2 m_{2}+m_{11}+m_{12}+m_{22}+m_{24}+m_{33}+m_{34}}\left(p_{3}-h_{2}+1-d / 2\right)_{m_{2}}} \\
& \times \frac{\left(-m_{12}^{\prime}\right)_{j_{1}+j_{2}+j_{3}+m_{12}}\left(p_{3}+h_{5}-m_{1}+m_{2}+m_{12}-m_{13}^{\prime}-m_{23}^{\prime}-m_{44}^{\prime}\right)_{m_{12}^{\prime}-m_{12}-j_{1}-j_{2}-j_{3}}}{\left(m_{13}-m_{13}^{\prime}-j_{1}\right) !\left(m_{23}-m_{23}^{\prime}-j_{2}\right) !}
\end{aligned}
$$

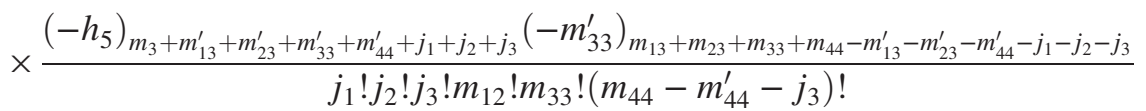

$$
\begin{aligned}
& \times(-1)^{j_{1}+j_{2}+j_{3}} F_{7} \prod_{1 \leq a \leq 4} \frac{\left(u_{a}^{7}\right)^{m_{a}}}{m_{a} !} \prod_{1 \leq a \leq b \leq 4} \frac{\left(1-v_{a b}^{7}\right)^{m_{a b}^{\prime}}}{m_{a b}^{\prime} !} .
\end{aligned}
$$

We then define $m_{13}=m-m_{44}$ and shift $m_{44}$ by $m_{44}+m_{44}^{\prime}+j_{3}$. This allows us to sum over $m_{44}$, leading to

$$
\begin{aligned}
& G_{7 P_{1}}=\sum \frac{\left(p_{4}-m_{1}\right)_{m_{4}+m_{14}}\left(-h_{6}\right)_{m_{4}+m_{44}^{\prime}+m_{14}+m_{24}+m_{34}+j_{3}}\left(p_{4}-h_{3}+h_{6}\right)_{m_{4}+m_{13}^{\prime}+m_{11}+m_{22}+j_{1}}}{\left(p_{4}-h_{3}\right)_{2 m_{4}+m_{13}^{\prime}+m_{44}^{\prime}+m_{11}+m_{14}+m_{22}+m_{24}+m_{34}+j_{1}+j_{3}}\left(p_{4}-h_{3}+1-d / 2\right)_{m_{4}}} \\
& \times \frac{\left(p_{2}+h_{3}\right)_{m_{1}-m_{4}+m_{23}}\left(-h_{4}\right)_{m_{2}+m_{11}+m_{34}}\left(\bar{p}_{3}+h_{2}+h_{5}\right)_{m_{3}+m_{12}}}{\left(p_{2}+h_{3}+m_{1}\right)_{-m_{4}}\left(\bar{p}_{3}+h_{2}\right)_{2 m_{3}+m_{12}+m_{23}+m_{33}+m}\left(\bar{p}_{3}+h_{2}+1-d / 2\right)_{m_{3}}} \\
& \times \frac{\left(p_{3}\right)_{-m_{1}+m_{2}+m_{3}+m_{12}+m_{33}}\left(p_{2}+h_{2}\right)_{m_{1}-m_{2}+m_{3}+m_{23}+m}\left(-h_{2}\right)_{m_{1}+m_{2}-m_{3}+m_{11}+m_{22}+m_{24}+m_{34}}}{\left(p_{2}\right)_{2 m_{1}+m_{11}+m_{22}+m_{23}+m_{24}+m_{34}+m}\left(p_{2}+1-d / 2\right)_{m_{1}}}
\end{aligned}
$$

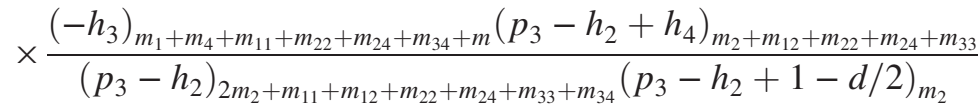

$$
\begin{aligned}
& \times \frac{\left(-m_{12}^{\prime}\right)_{j_{1}+j_{2}+j_{3}+m_{12}}\left(p_{3}+h_{5}-m_{1}+m_{2}+m_{12}-m_{13}^{\prime}-m_{23}^{\prime}-m_{44}^{\prime}\right)_{m_{12}^{\prime}-m_{12}-j_{1}-j_{2}-j_{3}}}{\left(m-m_{13}^{\prime}-m_{44}^{\prime}-j_{1}-j_{3}\right) !\left(m_{23}-m_{23}^{\prime}-j_{2}\right) !}
\end{aligned}
$$

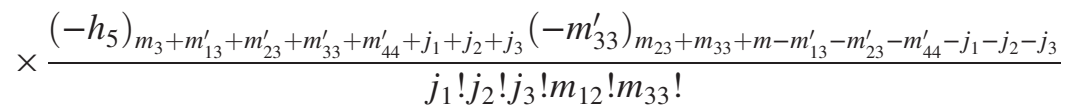

$$
\begin{aligned}
& \times(-1)^{j_{1}+j_{2}+j_{3}} F_{7} \prod_{1 \leq a \leq 4} \frac{\left(u_{a}^{7}\right)^{m_{a}}}{m_{a} !} \prod_{1 \leq a \leq b \leq 4} \frac{\left(1-v_{a b}^{7}\right)^{m_{a b}^{\prime}}}{m_{a b}^{\prime} !} .
\end{aligned}
$$


Defining $j_{3}=j-j_{1}$, the sum over $j_{1}$ thus gives

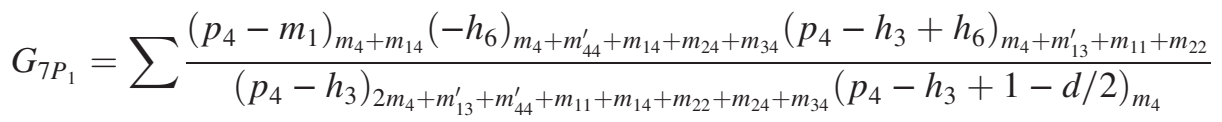

$$
\begin{aligned}
& \times \frac{\left(p_{2}+h_{3}\right)_{m_{1}-m_{4}+m_{23}}\left(-h_{4}\right)_{m_{2}+m_{11}+m_{34}}\left(\bar{p}_{3}+h_{2}+h_{5}\right)_{m_{3}+m_{12}}}{\left(p_{2}+h_{3}+m_{1}\right)_{-m_{4}}\left(\bar{p}_{3}+h_{2}\right)_{2 m_{3}+m_{12}+m_{23}+m_{33}+m}\left(\bar{p}_{3}+h_{2}+1-d / 2\right)_{m_{3}}} \\
& \times \frac{\left(p_{3}\right)_{-m_{1}+m_{2}+m_{3}+m_{12}+m_{33}}\left(p_{2}+h_{2}\right)_{m_{1}-m_{2}+m_{3}+m_{23}+m}\left(-h_{2}\right)_{m_{1}+m_{2}-m_{3}+m_{11}+m_{22}+m_{24}+m_{34}}}{\left(p_{2}\right)_{2 m_{1}+m_{11}+m_{22}+m_{23}+m_{24}+m_{34}+m}\left(p_{2}+1-d / 2\right)_{m_{1}}}
\end{aligned}
$$

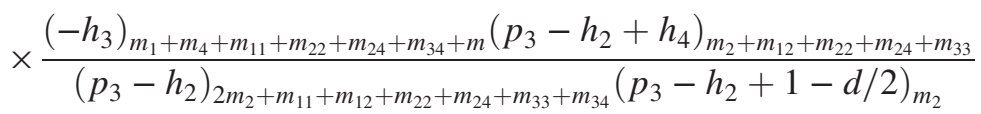

$$
\begin{aligned}
& \times \frac{\left(-m_{12}^{\prime}\right)_{j+j_{2}+m_{12}}\left(p_{3}+h_{5}-m_{1}+m_{2}+m_{12}-m_{13}^{\prime}-m_{23}^{\prime}-m_{44}^{\prime}\right)_{m_{12}^{\prime}-m_{12}-j-j_{2}}}{\left(m-m_{13}^{\prime}-m_{44}^{\prime}-j\right) !\left(m_{23}-m_{23}^{\prime}-j_{2}\right) !} \\
& \times \frac{\left(-h_{5}\right)_{m_{3}+m_{13}^{\prime}+m_{23}^{\prime}+m_{33}^{\prime}+m_{44}^{\prime}+j+j_{2}}\left(-m_{33}^{\prime}\right)_{m_{23}+m_{33}+m-m_{13}^{\prime}-m_{23}^{\prime}-m_{44}^{\prime}-j-j_{2}}}{j ! j_{2} ! m_{12} ! m_{33} !} \\
& \times(-1)^{j+j_{2}} F_{7} \prod_{1 \leq a \leq 4} \frac{\left(u_{a}^{7}\right)^{m_{a}}}{m_{a} !} \prod_{1 \leq a \leq b \leq 4} \frac{\left(1-v_{a b}^{7}\right)^{m_{a b}^{\prime}}}{m_{a b}^{\prime} !} .
\end{aligned}
$$

We now define $m_{23}=k-m$ and then change $m$ by $m \rightarrow m+m_{13}^{\prime}+m_{44}^{\prime}+j$. The sums over $m$ and $k$ (with the extra change $k \rightarrow k+m_{13}^{\prime}+m_{23}^{\prime}+m_{44}^{\prime}+j+j_{2}$ ) can be performed and lead to

$$
\begin{aligned}
& G_{7 P_{1}}=\sum \frac{\left(p_{4}-m_{1}\right)_{m_{4}+m_{14}}\left(-h_{6}\right)_{m_{4}+m_{44}^{\prime}+m_{14}+m_{24}+m_{34}}\left(p_{4}-h_{3}+h_{6}\right)_{m_{4}+m_{13}^{\prime}+m_{11}+m_{22}}}{\left(p_{4}-h_{3}\right)_{2 m_{4}+m_{13}^{\prime}+m_{44}^{\prime}+m_{11}+m_{14}+m_{22}+m_{24}+m_{34}}\left(p_{4}-h_{3}+1-d / 2\right)_{m_{4}}} \\
& \times \frac{\left(p_{2}+h_{3}\right)_{m_{1}-m_{4}+m_{23}^{\prime}+j_{2}}\left(-h_{4}\right)_{m_{2}+m_{11}+m_{34}}\left(\bar{p}_{3}+h_{2}+h_{5}\right)_{m_{3}+m_{12}}}{\left(p_{2}+h_{3}+m_{1}\right)_{-m_{4}}\left(\bar{p}_{3}+h_{2}\right)_{2 m_{3}+m_{12}+m_{13}^{\prime}+m_{23}^{\prime}+m_{33}^{\prime}+m_{44}^{\prime}+j+j_{2}}\left(\bar{p}_{3}+h_{2}+1-d / 2\right)_{m_{3}}} \\
& \times \frac{\left(p_{3}\right)_{-m_{1}+m_{2}+m_{3}+m_{12}+m_{33}^{\prime}}\left(p_{2}+h_{2}\right)_{m_{1}-m_{2}+m_{3}+m_{13}^{\prime}+m_{23}^{\prime}+m_{44}^{\prime}+j+j_{2}}\left(-h_{2}\right)_{m_{1}+m_{2}-m_{3}+m_{11}+m_{22}+m_{24}+m_{34}}}{\left(p_{2}\right)_{2 m_{1}+m_{13}^{\prime}+m_{23}^{\prime}+m_{44}^{\prime}+m_{11}+m_{22}+m_{24}+m_{34}+j+j_{2}}\left(p_{2}+1-d / 2\right)_{m_{1}}}
\end{aligned}
$$

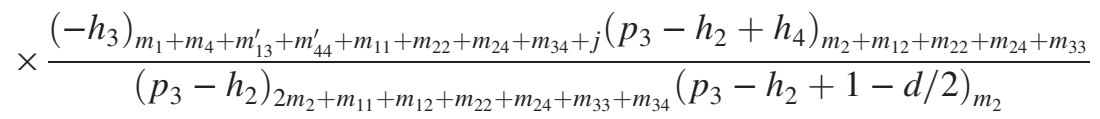

$$
\begin{aligned}
& \times \frac{\left(-m_{33}^{\prime}\right)_{m_{33}}\left(-m_{12}^{\prime}\right)_{j+j_{2}+m_{12}}\left(p_{3}+h_{5}-m_{1}+m_{2}+m_{12}-m_{13}^{\prime}-m_{23}^{\prime}-m_{44}^{\prime}\right)_{m_{12}^{\prime}-m_{12}-j-j_{2}}}{j ! j_{2} ! m_{12} ! m_{33} !} \\
& \times\left(-h_{5}\right)_{m_{3}+m_{13}^{\prime}+m_{23}^{\prime}+m_{33}^{\prime}+m_{44}^{\prime}+j+j_{2}}(-1)^{j+j_{2}} F_{7} \prod_{1 \leq a \leq 4} \frac{\left(u_{a}^{7}\right)^{m_{a}}}{m_{a} !} \prod_{1 \leq a \leq b \leq 4} \frac{\left(1-v_{a b}^{7}\right)^{m_{a b}^{\prime}}}{m_{a b}^{\prime} !} .
\end{aligned}
$$


It is now possible to resum over $m_{33}$ to get

$$
\begin{aligned}
& G_{7 P_{1}}=\sum \frac{\left(p_{4}-m_{1}\right)_{m_{4}+m_{14}}\left(-h_{6}\right)_{m_{4}+m_{44}^{\prime}+m_{14}+m_{24}+m_{34}}\left(p_{4}-h_{3}+h_{6}\right)_{m_{4}+m_{13}^{\prime}+m_{11}+m_{22}}}{\left(p_{4}-h_{3}\right)_{2 m_{4}+m_{13}^{\prime}+m_{44}^{\prime}+m_{11}+m_{14}+m_{22}+m_{24}+m_{34}}\left(p_{4}-h_{3}+1-d / 2\right)_{m_{4}}} \\
& \times \frac{\left(p_{2}+h_{3}\right)_{m_{1}-m_{4}+m_{23}^{\prime}+j_{2}}\left(-h_{4}\right)_{m_{2}+m_{11}+m_{34}+m_{33}^{\prime}}\left(\bar{p}_{3}+h_{2}+h_{5}\right)_{m_{3}+m_{12}}}{\left(p_{2}+h_{3}+m_{1}\right)_{-m_{4}}\left(\bar{p}_{3}+h_{2}\right)_{2 m_{3}+m_{12}+m_{13}^{\prime}+m_{23}^{\prime}+m_{33}^{\prime}+m_{44}^{\prime}+j+j_{2}}\left(\bar{p}_{3}+h_{2}+1-d / 2\right)_{m_{3}}} \\
& \times \frac{\left(p_{3}\right)_{-m_{1}+m_{2}+m_{3}+m_{12}+m_{33}^{\prime}}\left(p_{2}+h_{2}\right)_{m_{1}-m_{2}+m_{3}+m_{13}^{\prime}+m_{23}^{\prime}+m_{44}^{\prime}+j+j_{2}}\left(-h_{2}\right)_{m_{1}+m_{2}-m_{3}+m_{11}+m_{22}+m_{24}+m_{34}}}{\left(p_{2}\right)_{2 m_{1}+m_{13}^{\prime}+m_{23}^{\prime}+m_{44}^{\prime}+m_{11}+m_{22}+m_{24}+m_{34}+j+j_{2}}\left(p_{2}+1-d / 2\right)_{m_{1}}} \\
& \times \frac{\left(-h_{3}\right)_{m_{1}+m_{4}+m_{13}^{\prime}+m_{44}^{\prime}+m_{11}+m_{22}+m_{24}+m_{34}+j}\left(p_{3}-h_{2}+h_{4}\right)_{m_{2}+m_{12}+m_{22}+m_{24}}}{\left(p_{3}-h_{2}\right)_{2 m_{2}+m_{11}+m_{12}+m_{22}+m_{24}+m_{33}^{\prime}+m_{34}}\left(p_{3}-h_{2}+1-d / 2\right)_{m_{2}}} \\
& \times \frac{\left(-m_{12}^{\prime}\right)_{j+j_{2}+m_{12}}\left(p_{3}+h_{5}-m_{1}+m_{2}+m_{12}-m_{13}^{\prime}-m_{23}^{\prime}-m_{44}^{\prime}\right)_{m_{12}^{\prime}-m_{12}-j-j_{2}}}{j ! j_{2} ! m_{12} !} \\
& \times\left(-h_{5}\right)_{m_{3}+m_{13}^{\prime}+m_{23}^{\prime}+m_{33}^{\prime}+m_{44}^{\prime}+j+j_{2}}(-1)^{j+j_{2}} F_{7} \prod_{1 \leq a \leq 4} \frac{\left(u_{a}^{7}\right)^{m_{a}}}{m_{a} !} \prod_{1 \leq a \leq b \leq 4} \frac{\left(1-v_{a b}^{7}\right)^{m_{a b}^{\prime}}}{m_{a b}^{\prime} !} .
\end{aligned}
$$

We finally shift $j$ by $j \rightarrow j-j_{2}$ and evaluate the sums over $j_{2}, j$, and $m_{12},{ }^{9}$ to obtain

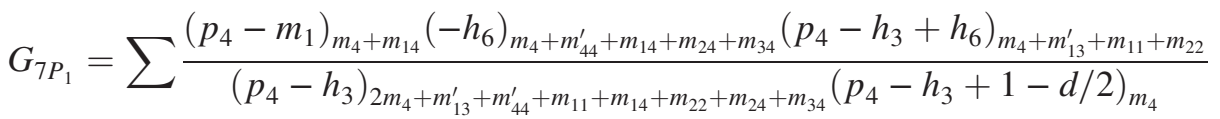

$$
\begin{aligned}
& \times \frac{\left(p_{2}+h_{3}\right)_{m_{1}-m_{4}+m_{23}^{\prime}}\left(-h_{4}\right)_{m_{2}+m_{11}+m_{34}+m_{12}^{\prime}+m_{33}^{\prime}}\left(\bar{p}_{3}+h_{2}+h_{5}\right)_{m_{3}+m_{12}^{\prime}}}{\left(p_{2}+h_{3}+m_{1}\right)_{-m_{4}}\left(\bar{p}_{3}+h_{2}\right)_{2 m_{3}+m_{12}^{\prime}+m_{13}^{\prime}+m_{23}^{\prime}+m_{33}^{\prime}+m_{44}^{\prime}}\left(\bar{p}_{3}+h_{2}+1-d / 2\right)_{m_{3}}}
\end{aligned}
$$

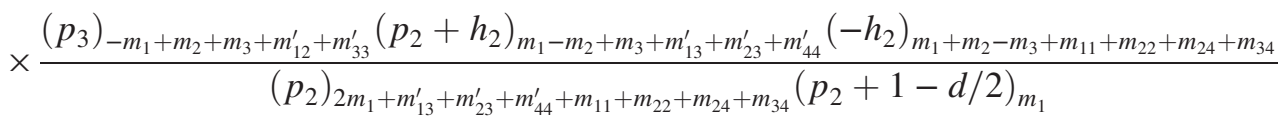

$$
\begin{aligned}
& \times \frac{\left(-h_{3}\right)_{m_{1}+m_{4}+m_{13}^{\prime}+m_{44}^{\prime}+m_{11}+m_{22}+m_{24}+m_{34}}\left(p_{3}-h_{2}+h_{4}\right)_{m_{2}+m_{22}+m_{24}}\left(-h_{5}\right)_{m_{3}+m_{13}^{\prime}+m_{23}^{\prime}+m_{33}^{\prime}+m_{44}^{\prime}}}{\left(p_{3}-h_{2}\right)_{2 m_{2}+m_{11}+m_{12}^{\prime}+m_{22}+m_{24}+m_{33}^{\prime}+m_{34}}\left(p_{3}-h_{2}+1-d / 2\right)_{m_{2}}} \\
& \times F_{7} \prod_{1 \leq a \leq 4} \frac{\left(u_{a}^{7}\right)^{m_{a}}}{m_{a} !} \prod_{1 \leq a \leq b \leq 4} \frac{\left(1-v_{a b}^{7}\right)^{m_{a b}^{\prime}}}{m_{a b}^{\prime} !} \text {. }
\end{aligned}
$$

Using the fact that $m_{11}=m_{22}^{\prime}, m_{22}=m_{11}^{\prime}, m_{14}=m_{14}^{\prime}, m_{24}=m_{34}^{\prime}$ and $m_{34}=m_{24}^{\prime}$, we find that $G_{7 P}=G_{7}$ as expected from (3.2), proving invariance of the scalar seven-point conformal blocks in the extended snowflake channel under dendrite permutations of the first kind.

\section{Dendrite permutations of the second kind}

We finally focus on the proof of the invariance of the scalar seven-point correlation functions under dendrite permutations of the second kind (3.3), which we write as $G_{7}=G_{7 P_{2}}$ for notational simplicity.

\footnotetext{
${ }^{9}$ Before proceeding with the sum over $j$, we use the first identity in (A.3).
} 
Taking into account the fact that $F_{7}$ is invariant under dendrite permutations of the second kind, we find that

$$
\begin{aligned}
& G_{7 P_{2}}=\sum \frac{\left(p_{4}-m_{1}\right)_{m_{4}+m_{14}}\left(p_{4}-h_{3}+h_{6}\right)_{m_{4}+m_{14}+m_{24}+m_{34}+m_{44}}\left(-h_{6}\right)_{m_{4}+m_{11}+m_{13}+m_{22}}}{\left(p_{2}+h_{3}+m_{1}\right)_{-m_{4}}\left(p_{4}-h_{3}\right)_{2 m_{4}+m_{11}+m_{13}+m_{14}+m_{22}+m_{24}+m_{34}+m_{44}}\left(p_{4}-h_{3}+1-d / 2\right)_{m_{4}}} \\
& \times \frac{\left(p_{2}+h_{3}\right)_{m_{1}-m_{4}+m_{23}}\left(p_{3}\right)_{-m_{1}+m_{2}+m_{3}+m_{12}+m_{33}}\left(p_{3}-h_{2}+h_{4}\right)_{m_{2}+m_{11}+m_{34}}\left(\bar{p}_{3}+h_{2}+h_{5}\right)_{m_{3}+m_{12}}}{\left(\bar{p}_{3}+h_{2}\right)_{2 m_{3}+m_{12}+m_{13}+m_{23}+m_{33}+m_{44}}\left(\bar{p}_{3}+h_{2}+1-d / 2\right)_{m_{3}}} \\
& \times \frac{\left(p_{2}+h_{2}\right)_{m_{1}-m_{2}+m_{3}+m_{13}+m_{23}+m_{44}}\left(-h_{2}\right)_{m_{1}+m_{2}-m_{3}+m_{11}+m_{22}+m_{24}+m_{34}}}{\left(p_{2}\right)_{2 m_{1}+m_{11}+m_{13}+m_{22}+m_{23}+m_{24}+m_{34}+m_{44}\left(p_{2}+1-d / 2\right)_{m_{1}}}}
\end{aligned}
$$

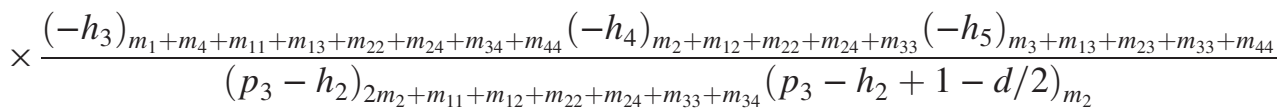

$$
\begin{aligned}
& \times(-1)^{k_{14}+m_{14}^{\prime}} \frac{m_{14}^{\prime} !}{m_{14} !}\left(\begin{array}{c}
m_{14} \\
k_{14}
\end{array}\right)\left(\begin{array}{c}
-p_{4}+m_{1}-m_{4}-k_{14} \\
m_{14}^{\prime}
\end{array}\right) F_{7} \prod_{1 \leq a \leq 4} \frac{\left(u_{a}^{7}\right)^{m_{a}}}{m_{a} !} \prod_{1 \leq a \leq b \leq 4} \frac{\left(1-v_{a b}^{7}\right)^{m_{a b}^{\prime}}}{m_{a b}^{\prime} !},
\end{aligned}
$$

with

$$
\begin{array}{lll}
m_{11}=m_{34}^{\prime}, & m_{12}=m_{12}^{\prime}, & m_{22}=m_{24}^{\prime}, \\
m_{13}=m_{44}^{\prime}, & m_{23}=m_{23}^{\prime}, & m_{33}=m_{33}^{\prime}, \\
m_{24}=m_{22}^{\prime}, & m_{34}=m_{11}^{\prime}, & m_{44}=m_{13}^{\prime} .
\end{array}
$$

Computing the sum over $k_{14}$ leads to

$$
\begin{aligned}
& G_{7 P_{2}}=\sum \frac{\left(p_{4}-m_{1}\right)_{m_{4}+m_{14}^{\prime}}\left(p_{4}-h_{3}+h_{6}\right)_{m_{4}+m_{14}+m_{24}+m_{34}+m_{44}}\left(-h_{6}\right)_{m_{4}+m_{11}+m_{13}+m_{22}}}{\left(p_{2}+h_{3}+m_{1}\right)_{-m_{4}}\left(p_{4}-h_{3}\right)_{2 m_{4}+m_{11}+m_{13}+m_{14}+m_{22}+m_{24}+m_{34}+m_{44}}\left(p_{4}-h_{3}+1-d / 2\right)_{m_{4}}} \\
& \times \frac{\left(p_{2}+h_{3}\right)_{m_{1}-m_{4}+m_{23}}\left(p_{3}\right)_{-m_{1}+m_{2}+m_{3}+m_{12}+m_{33}}\left(p_{3}-h_{2}+h_{4}\right)_{m_{2}+m_{11}+m_{34}}\left(\bar{p}_{3}+h_{2}+h_{5}\right)_{m_{3}+m_{12}}}{\left(\bar{p}_{3}+h_{2}\right)_{2 m_{3}+m_{12}+m_{13}+m_{23}+m_{33}+m_{44}}\left(\bar{p}_{3}+h_{2}+1-d / 2\right)_{m_{3}}}
\end{aligned}
$$

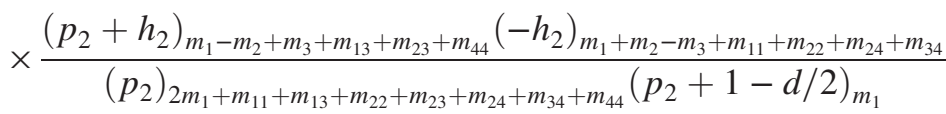

$$
\begin{aligned}
& \times \frac{\left(-h_{3}\right)_{m_{1}+m_{4}+m_{11}+m_{13}+m_{22}+m_{24}+m_{34}+m_{44}}\left(-h_{4}\right)_{m_{2}+m_{12}+m_{22}+m_{24}+m_{33}}\left(-h_{5}\right)_{m_{3}+m_{13}+m_{23}+m_{33}+m_{44}}}{\left(p_{3}-h_{2}\right)_{2 m_{2}+m_{14}+m_{12}+m_{22}+m_{24}+m_{33}+m_{34}}\left(p_{3}-h_{2}+1-d / 2\right)_{m_{2}}} \\
& \times \frac{\left(-m_{14}^{\prime}\right)_{m_{14}}}{m_{14} !} F_{7} \prod_{1 \leq a \leq 4} \frac{\left(u_{a}^{7}\right)^{m_{a}}}{m_{a} !} \prod_{1 \leq a \leq b \leq 4} \frac{\left(1-v_{a b}^{7}\right)^{m_{a b}^{\prime}}}{m_{a b}^{\prime} !} .
\end{aligned}
$$

We then evaluate the sum over $m_{14}$, giving

$$
\begin{aligned}
& G_{7 P_{2}}=\sum \frac{\left(p_{4}-m_{1}\right)_{m_{4}+m_{14}^{\prime}}\left(p_{4}-h_{3}+h_{6}\right)_{m_{4}+m_{24}+m_{34}+m_{44}}\left(-h_{6}\right)_{m_{4}+m_{11}+m_{13}+m_{14}^{\prime}+m_{22}}}{\left(p_{2}+h_{3}+m_{1}\right)_{-m_{4}}\left(p_{4}-h_{3}\right)_{2 m_{4}+m_{11}+m_{13}+m_{14}^{\prime}+m_{22}+m_{24}+m_{34}+m_{44}}\left(p_{4}-h_{3}+1-d / 2\right)_{m_{4}}} \\
& \times \frac{\left(p_{2}+h_{3}\right)_{m_{1}-m_{4}+m_{23}}\left(p_{3}\right)_{-m_{1}+m_{2}+m_{3}+m_{12}+m_{33}}\left(p_{3}-h_{2}+h_{4}\right)_{m_{2}+m_{11}+m_{34}}\left(\bar{p}_{3}+h_{2}+h_{5}\right)_{m_{3}+m_{12}}}{\left(\bar{p}_{3}+h_{2}\right)_{2 m_{3}+m_{12}+m_{13}+m_{23}+m_{33}+m_{44}}\left(\bar{p}_{3}+h_{2}+1-d / 2\right)_{m_{3}}} \\
& \times \frac{\left(p_{2}+h_{2}\right)_{m_{1}-m_{2}+m_{3}+m_{13}+m_{23}+m_{44}}\left(-h_{2}\right)_{m_{1}+m_{2}-m_{3}+m_{11}+m_{22}+m_{24}+m_{34}}}{\left(p_{2}\right)_{2 m_{1}+m_{11}+m_{13}+m_{22}+m_{23}+m_{24}+m_{34}+m_{44}\left(p_{2}+1-d / 2\right)_{m_{1}}}} \\
& \times \frac{\left(-h_{3}\right)_{m_{1}+m_{4}+m_{11}+m_{13}+m_{22}+m_{24}+m_{34}+m_{44}}\left(-h_{4}\right)_{m_{2}+m_{12}+m_{22}+m_{24}+m_{33}}\left(-h_{5}\right)_{m_{3}+m_{13}+m_{23}+m_{33}+m_{44}}}{\left(p_{3}-h_{2}\right)_{2 m_{2}+m_{11}+m_{12}+m_{22}+m_{24}+m_{33}+m_{34}}\left(p_{3}-h_{2}+1-d / 2\right)_{m_{2}}} \\
& \times F_{7} \prod_{1 \leq a \leq 4} \frac{\left(u_{a}^{7}\right)^{m_{a}}}{m_{a} !} \prod_{1 \leq a \leq b \leq 4} \frac{\left(1-v_{a b}^{7}\right)^{m_{a b}^{\prime}}}{m_{a b}^{\prime} !} \\
& =G_{7} \text {, }
\end{aligned}
$$


which ends the proof of the invariance of the scalar seven-point correlation functions under dendrite permutations of the second kind (3.3).

\section{APPENDIX C: OPE LIMIT AND LIMIT OF UNIT OPERATOR}

This Appendix presents the remaining proofs for the OPE limit as well as the limit of unit operator. As usual, all resummations are performed with the help of (A.1), (A.2) and (A.3).

\section{OPE limit}

In the OPE limit $\eta_{3} \rightarrow \eta_{4}$, we expect from the topologies that

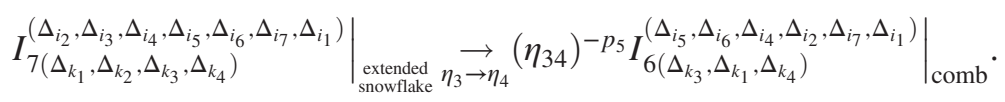

Defining all quantities in the vectors $\boldsymbol{h}$ and $\boldsymbol{p}$ on the rhs of (C.1) with primes, this leads to

$$
\begin{array}{rlrl}
L_{7} \prod_{1 \leq a \leq 4}\left(u_{a}^{7}\right)^{\frac{\Delta_{k a}}{2}} & \rightarrow\left(v_{23}^{6}\right)^{-\bar{p}_{4}^{\prime}-\bar{h}_{5}^{\prime}}\left(v_{12}^{6}\right)^{h_{5}^{\prime}} L_{6} \prod_{1 \leq a \leq 3}\left(u_{a}^{6}\right)^{\frac{\Delta_{k a}^{\prime}}{2}}, \\
u_{1}^{7} & \rightarrow \frac{u_{2}^{6}}{v_{23}^{6}}, \quad u_{2}^{7} \rightarrow 0, \quad u_{3}^{7} \rightarrow u_{1}^{6}, \quad u_{4}^{7} \rightarrow \frac{u_{3}^{6}}{v_{12}^{6}}, \\
v_{11}^{7} & \rightarrow \frac{v_{13}^{6}}{v_{23}^{6}}, & v_{12}^{7} & \rightarrow 1, \quad v_{13}^{7} \rightarrow \frac{v_{33}^{6}}{v_{23}^{6}}, \quad v_{14}^{7} \rightarrow \frac{v_{23}^{6}}{v_{12}^{6},} \\
v_{22}^{7} & \rightarrow \frac{v_{13}^{6}}{v_{23}^{6}}, & v_{23}^{7} & \rightarrow v_{11}^{6}, \quad v_{24}^{7} \rightarrow \frac{1}{v_{12}^{6}}, \quad v_{33}^{7} \rightarrow 1, \\
v_{34}^{7} & \rightarrow \frac{1}{v_{12}^{6}}, & v_{44}^{7} & \rightarrow \frac{v_{22}^{6}}{v_{12}^{6}},
\end{array}
$$

which implies the identity,

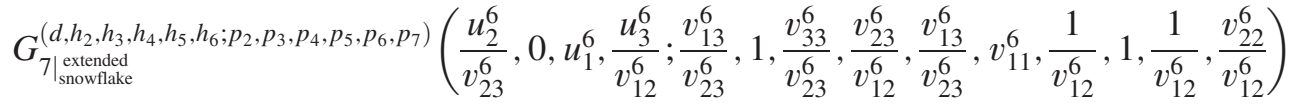

$$
\begin{aligned}
& =\left(v_{23}^{6}\right)^{\bar{p}_{4}^{\prime}+\bar{h}_{5}^{\prime}}\left(v_{12}^{6}\right)^{-h_{5}^{\prime}} G_{6 \mid \mathrm{comb}}^{\left(d, h_{2}^{\prime}, h_{3}^{\prime}, h_{4}^{\prime}, h_{5}^{\prime} ; p_{2}^{\prime}, p_{3}^{\prime}, p_{4}^{\prime}, p_{5}^{\prime}, p_{6}^{\prime}\right)}\left(u_{1}^{6}, u_{2}^{6}, u_{3}^{6} ; v_{11}^{6}, v_{12}^{6}, v_{13}^{6}, v_{22}^{6}, v_{23}^{6}, v_{33}^{6}\right) \text {. }
\end{aligned}
$$

Here the scalar six-point conformal blocks in the comb channel is given by

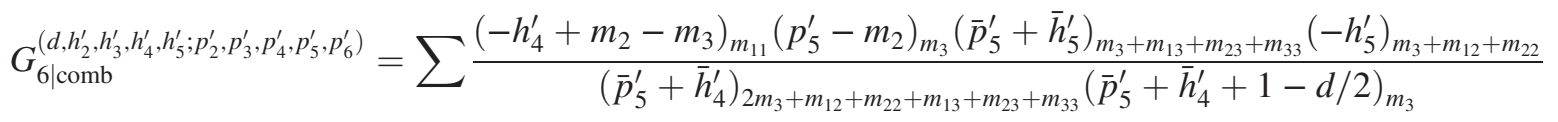

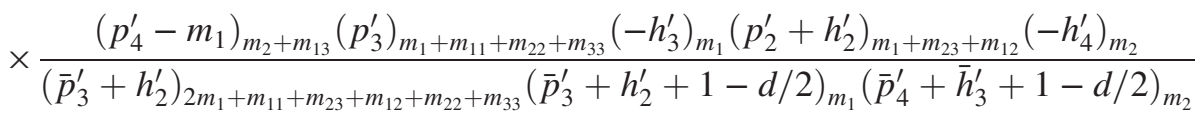

$$
\begin{aligned}
& \times \frac{\left(\bar{p}_{3}^{\prime}+\bar{h}_{3}^{\prime}\right)_{m_{1}+m_{2}+m_{11}+m_{23}+m_{12}+m_{22}+m_{33}}\left(\bar{p}_{4}^{\prime}+\bar{h}_{4}^{\prime}\right)_{m_{2}+m_{3}+m_{12}+m_{22}+m_{13}+m_{23}+m_{33}}}{\left(\bar{p}_{4}^{\prime}+\bar{h}_{3}^{\prime}\right)_{2 m_{2}+m_{11}+m_{12}+m_{22}+m_{13}+m_{23}+m_{33}}} \\
& \times F_{6} \prod_{1 \leq a \leq 3} \frac{\left(u_{a}^{6}\right)^{m_{a}}}{\left(m_{a}\right) !} \prod_{1 \leq a \leq b \leq 3} \frac{\left(1-v_{a b}^{6}\right)^{m_{a b}}}{\left(m_{a b}\right) !} \text {, }
\end{aligned}
$$

with

$$
F_{6}={ }_{3} F_{2}\left[\begin{array}{c}
-m_{1},-m_{2},-\bar{p}_{3}^{\prime}-\bar{h}_{2}^{\prime}+d / 2-m_{1} ; \\
p_{4}^{\prime}-m_{1}, h_{3}^{\prime}+1-m_{1}
\end{array}\right]{ }_{3} F_{2}\left[\begin{array}{c}
-m_{2},-m_{3},-\bar{p}_{4}^{\prime}-\bar{h}_{3}^{\prime}+d / 2-m_{2} ; \\
p_{5}^{\prime}-m_{2}, h_{4}^{\prime}+1-m_{2}
\end{array}\right],
$$


and

$$
\begin{aligned}
h_{2} & \rightarrow-p_{4}^{\prime}, \quad h_{3} \rightarrow-\bar{p}_{4}^{\prime}-\bar{h}_{4}^{\prime}, \quad h_{5} \rightarrow-p_{3}^{\prime}, \quad h_{6} \rightarrow h_{5}^{\prime}, \\
p_{2} & \rightarrow \bar{p}_{4}^{\prime}+\bar{h}_{3}^{\prime}, \quad p_{3} \rightarrow-h_{3}^{\prime}, \quad p_{4} \rightarrow p_{5}^{\prime}, \\
p_{2}+h_{3} & \rightarrow-h_{4}^{\prime}, \quad \bar{p}_{3}+h_{2}+h_{5} \rightarrow p_{2}^{\prime}+h_{2}^{\prime}, \quad p_{4}-h_{3}+h_{6} \rightarrow \bar{p}_{5}^{\prime}+\bar{h}_{5}^{\prime}, \\
p_{2}+h_{2} & \rightarrow \bar{p}_{3}^{\prime}+\bar{h}_{3}^{\prime}, \quad \bar{p}_{3}+\bar{h}_{2} \rightarrow \bar{p}_{3}^{\prime}+\bar{h}_{2}^{\prime}, \quad p_{4}-h_{3} \rightarrow \bar{p}_{5}^{\prime}+\bar{h}_{4}^{\prime} .
\end{aligned}
$$

To prove (C.1), which we rewrite as $G_{7 \mid \eta_{3} \rightarrow \eta_{4}}=G_{6}$ for simplicity, we first note that

$$
\begin{aligned}
F_{7} & =\frac{\left(-\bar{p}_{3}-h_{2}+d / 2-m_{1}^{\prime}\right)_{m_{1}^{\prime}}}{\left(p_{3}\right)_{-m_{2}^{\prime}}} \sum \frac{\left(-m_{1}^{\prime}\right)_{t_{2}}\left(\bar{p}_{3}-d / 2\right)_{t_{2}}\left(p_{3}\right)_{t_{2}}}{t_{2} !\left(p_{3}-m_{2}^{\prime}\right)_{t_{2}}\left(\bar{p}_{3}+h_{2}+1-d / 2\right)_{t_{2}}} \frac{\left(-m_{2}^{\prime}\right)_{t_{3}}\left(-m_{3}^{\prime}\right)_{t_{3}}\left(-p_{2}+d / 2-m_{2}^{\prime}\right)_{t_{3}}}{\left.t_{3}-h_{3}-m_{2}^{\prime}\right)_{t_{3}}\left(p_{4}-m_{2}^{\prime}\right)_{t_{3}}} \\
& =\frac{\left(-h_{2}-m_{1}^{\prime}\right)_{m_{1}^{\prime}}}{\left(p_{3}\right)_{-m_{2}^{\prime}}} \sum \frac{\left(-m_{1}^{\prime}\right)_{t_{2}}\left(-m_{2}^{\prime}\right)_{t_{2}}\left(\bar{p}_{3}-d / 2\right)_{t_{2}}}{t_{2} !\left(p_{3}-m_{2}^{\prime}\right)_{t_{3}}\left(-m_{3}^{\prime}\right)_{t_{3}}\left(-p_{2}-p_{2}+d / 2-m_{2}^{\prime}\right)_{t_{2}}} \frac{\left(-h_{2}-m_{1}^{\prime}\right)_{m_{1}^{\prime}}\left(p_{3}\right)_{m_{1}^{\prime}}}{t_{3} !\left(1-p_{2}-h_{3}-m_{2}^{\prime}\right)_{t_{3}}\left(p_{4}-m_{2}^{\prime}\right)_{t_{3}}} \sum \frac{\left(-m_{1}^{\prime}\right)_{t_{2}}\left(-m_{2}^{\prime}\right)_{t_{2}}\left(-\bar{p}_{3}-h_{2}+d / 2-m_{1}^{\prime}\right)_{t_{2}}}{t_{2} !\left(1-p_{3}-m_{1}^{\prime}\right)_{t_{2}}\left(-h_{2}-m_{1}^{\prime}\right)_{t_{2}}} \frac{\left(-m_{3}^{\prime}\right)_{t_{3}}\left(-p_{2}+d / 2-m_{2}^{\prime}\right)_{t_{3}}}{t_{3} !\left(1-p_{2}-h_{3}-m_{2}^{\prime}\right)_{t_{3}}\left(p_{4}-m_{2}^{\prime}\right)_{t_{3}}} \\
& =\frac{\left(-h_{2}-m_{1}^{\prime}\right)_{m_{1}^{\prime}}\left(p_{3}\right)_{m_{1}^{\prime}}}{\left(p_{3}\right)_{m_{1}^{\prime}-m_{2}^{\prime}}} F_{6} .
\end{aligned}
$$

Thus, multiplying $G_{7}$ by $\left(v_{23}^{6}\right)^{h_{3}-h_{6}}\left(v_{12}^{6}\right)^{h_{6}}$ and taking the OPE limit $\eta_{3} \rightarrow \eta_{4}$, we need to recover $G_{6}$ from

$$
\begin{aligned}
G_{7 \mid \eta_{3} \rightarrow \eta_{4}}= & \sum \frac{\left(p_{4}-m_{2}^{\prime}\right)_{m_{3}^{\prime}+m_{14}}\left(-h_{6}\right)_{m_{3}^{\prime}+m_{14}+m_{24}+m_{34}+m_{44}}\left(p_{4}-h_{3}+h_{6}\right)_{m_{3}^{\prime}+m_{11}+m_{13}+m_{22}}}{\left(p_{4}-h_{3}\right)_{2 m_{3}^{\prime}+m_{11}+m_{13}+m_{14}+m_{22}+m_{24}+m_{34}+m_{44}}\left(p_{4}-h_{3}+1-d / 2\right)_{m_{3}^{\prime}}} \\
& \times \frac{\left(p_{2}+h_{3}\right)_{m_{2}^{\prime}-m_{3}^{\prime}+m_{11}^{\prime}}\left(p_{3}-h_{2}+h_{4}\right)_{m_{11}+m_{34}}\left(\bar{p}_{3}+h_{2}+h_{5}\right)_{m_{1}^{\prime}}}{\left(p_{2}+h_{3}+m_{2}^{\prime}\right)_{-m_{3}^{\prime}}\left(\bar{p}_{3}+h_{2}\right)_{2 m_{1}^{\prime}+m_{13}+m_{44}+m_{11}^{\prime}}\left(\bar{p}_{3}+h_{2}+1-d / 2\right)_{m_{1}^{\prime}}} \\
& \times \frac{\left(p_{3}\right)_{m_{1}^{\prime}}\left(p_{2}+h_{2}\right)_{m_{2}^{\prime}+m_{1}^{\prime}+m_{13}+m_{44}+m_{11}^{\prime}}\left(-h_{2}-m_{1}^{\prime}\right)_{m_{1}^{\prime}}\left(-h_{2}\right)_{m_{2}^{\prime}-m_{1}^{\prime}+m_{11}+m_{22}+m_{24}+m_{34}}}{\left(p_{2}\right)_{2 m_{2}^{\prime}+m_{11}+m_{13}+m_{22}+m_{11}^{\prime}+m_{24}+m_{34}+m_{44}}\left(p_{2}+1-d / 2\right)_{m_{2}^{\prime}}} \\
& \times \frac{\left(-h_{3}\right)_{m_{2}^{\prime}+m_{3}^{\prime}+m_{11}+m_{13}+m_{22}+m_{24}+m_{34}+m_{44}}\left(-h_{4}\right)_{m_{22}+m_{24}}\left(-h_{5}\right)_{m_{1}^{\prime}+m_{13}+m_{11}^{\prime}+m_{44}}}{\left(p_{3}-h_{2}\right)_{m_{11}+m_{22}+m_{24}+m_{34}}} \\
& \times\left(\begin{array}{c}
m_{11} \\
k_{11}
\end{array}\right)\left(\begin{array}{c}
m_{13} \\
k_{13}
\end{array}\right)\left(\begin{array}{c}
m_{14} \\
k_{14}
\end{array}\right)\left(\begin{array}{c}
m_{22} \\
k_{22}
\end{array}\right)\left(\begin{array}{c}
m_{24} \\
k_{24}
\end{array}\right)\left(\begin{array}{c}
m_{34} \\
k_{34}
\end{array}\right)\left(\begin{array}{l}
m_{44} \\
k_{44}
\end{array}\right)\left(\begin{array}{c}
h_{6}-m_{3}^{\prime}-k_{14}-k_{24}-k_{34}-k_{44} \\
m_{33}^{\prime}
\end{array}\right) \\
& \times\left(\begin{array}{c}
k_{11} \\
l_{13}
\end{array}\right)\left(\begin{array}{c}
k_{22} \\
m_{13}^{\prime}-l_{13}
\end{array}\right)\left(\begin{array}{c}
k_{44} \\
m_{22}^{\prime}
\end{array}\right)\left(\begin{array}{l}
h_{3}-h_{6}-m_{2}^{\prime}-k_{11}-k_{13}-k_{22}+k_{14} \\
m_{23}^{\prime}
\end{array}\right. \\
& \times(-1)^{k_{11}+k_{13}+k_{14}+k_{22}+k_{24}+k_{34}+k_{44}+m_{12}^{\prime}+m_{13}^{\prime}+m_{22}^{\prime}+m_{23}^{\prime}+m_{33}^{\prime}} \\
& \times F_{6} \prod_{1 \leq a \leq 3} \frac{\left(u_{a}^{6}\right)^{m_{a}^{\prime}}}{m_{a}^{\prime} !} \frac{m_{12} ! m_{33} ! \prod_{1 \leq a \leq b \leq 3}\left(1-v_{a b}^{6}\right)_{a b}^{m_{a}^{\prime}}}{\prod_{1 \leq a \leq b \leq 4} m_{a b} !}
\end{aligned}
$$


To proceed, we first evaluate the sums over $k_{13}, k_{22}, k_{24}, k_{34}$, and finally $k_{44},{ }^{10}$ which gives

$$
\begin{aligned}
& G_{7 \mid \eta_{3} \rightarrow \eta_{4}}=\sum \frac{\left(p_{4}-m_{2}^{\prime}\right)_{m_{3}^{\prime}+m_{14}}\left(-h_{6}\right)_{m_{3}^{\prime}+m_{14}+m_{24}+m_{34}+m_{44}}\left(p_{4}-h_{3}+h_{6}\right)_{m_{3}^{\prime}+m_{11}+m_{13}+m_{22}}}{\left(p_{4}-h_{3}\right)_{2 m_{3}^{\prime}+m_{11}+m_{13}+m_{14}+m_{22}+m_{24}+m_{34}+m_{44}}\left(p_{4}-h_{3}+1-d / 2\right)_{m_{3}^{\prime}}} \\
& \times \frac{\left(p_{2}+h_{3}\right)_{m_{2}^{\prime}-m_{3}^{\prime}+m_{11}^{\prime}}\left(p_{3}-h_{2}+h_{4}\right)_{m_{11}+m_{34}}\left(\bar{p}_{3}+h_{2}+h_{5}\right)_{m_{1}^{\prime}}}{\left(p_{2}+h_{3}+m_{2}^{\prime}\right)_{-m_{3}^{\prime}}\left(\bar{p}_{3}+h_{2}\right)_{2 m_{1}^{\prime}+m_{13}+m_{44}+m_{11}^{\prime}}\left(\bar{p}_{3}+h_{2}+1-d / 2\right)_{m_{1}^{\prime}}} \\
& \times \frac{\left(p_{3}\right)_{m_{1}^{\prime}}\left(p_{2}+h_{2}\right)_{m_{2}^{\prime}+m_{1}^{\prime}+m_{13}+m_{44}+m_{11}^{\prime}}\left(-h_{2}-m_{1}^{\prime}\right)_{m_{1}^{\prime}}\left(-h_{2}\right)_{m_{2}^{\prime}-m_{1}^{\prime}+m_{11}+m_{22}+m_{24}+m_{34}}}{\left(p_{2}\right)_{2 m_{2}^{\prime}+m_{11}+m_{13}+m_{22}+m_{11}^{\prime}+m_{24}+m_{34}+m_{44}}\left(p_{2}+1-d / 2\right)_{m_{2}^{\prime}}} \\
& \times \frac{\left(-h_{3}\right)_{m_{2}^{\prime}+m_{3}^{\prime}+m_{11}+m_{13}+m_{22}+m_{24}+m_{34}+m_{44}}\left(-h_{4}\right)_{m_{22}+m_{24}}\left(-h_{5}\right)_{m_{1}^{\prime}+m_{13}+m_{11}^{\prime}+m_{44}}}{\left(p_{3}-h_{2}\right)_{m_{11}+m_{22}+m_{24}+m_{34}}}
\end{aligned}
$$

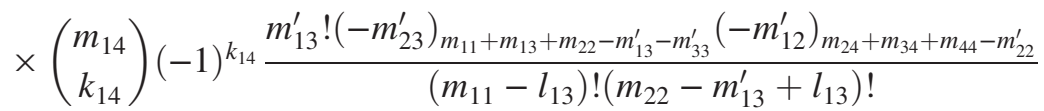

$$
\begin{aligned}
& \times \frac{\left(-h_{3}+h_{6}+m_{2}^{\prime}+m_{11}+m_{13}+m_{22}-k_{14}\right)_{m_{13}^{\prime}+m_{23}^{\prime}+m_{33}^{\prime}-m_{11}-m_{13}-m_{22}}}{\left(m_{13}-m_{33}^{\prime}\right) !} \\
& \times \frac{\left(-h_{6}+m_{3}^{\prime}+m_{24}+m_{34}+m_{44}+k_{14}\right)_{m_{12}^{\prime}+m_{22}^{\prime}-m_{24}-m_{34}-m_{44}}}{l_{13} !\left(m_{13}^{\prime}-l_{13}\right) !\left(m_{44}-m_{22}^{\prime}\right) ! m_{14} ! m_{24} ! m_{34} !} \frac{\left(u_{a}^{6}\right)^{m_{a}^{\prime}}}{m_{a}^{\prime} !} \prod_{1 \leq a \leq b \leq 3} \frac{\left(1-v_{a b}^{6}\right)^{m_{a b}^{\prime}}}{m_{a b}^{\prime} !} .
\end{aligned}
$$

To eliminate the sum over $k_{14}$, we now use the following identity:

$$
\begin{aligned}
& \left(-h_{3}+h_{6}+m_{2}^{\prime}+m_{11}+m_{13}+m_{22}-k_{14}\right)_{m_{13}^{\prime}+m_{23}^{\prime}+m_{33}^{\prime}-m_{11}-m_{13}-m_{22}} \\
& =\sum_{j}\left(\begin{array}{c}
m_{13}^{\prime}+m_{23}^{\prime}+m_{33}^{\prime}-m_{11}-m_{13}-m_{22} \\
j
\end{array}\right) \\
& \quad \times\left(-h_{3}+h_{6}+m_{2}^{\prime}+m_{11}+m_{13}+m_{22}\right)_{m_{13}^{\prime}+m_{23}^{\prime}+m_{33}^{\prime}-m_{11}-m_{13}-m_{22}-j}\left(-k_{14}\right)_{j},
\end{aligned}
$$

and then change the variable by $k_{14} \rightarrow k_{14}+j$. The sum over $k_{14}$ can be performed, leading to

$$
\begin{aligned}
& G_{7 \mid \eta_{3} \rightarrow \eta_{4}}=\sum \frac{\left(p_{4}-m_{2}^{\prime}\right)_{m_{3}^{\prime}+m_{14}}\left(-h_{6}\right)_{m_{3}^{\prime}+m_{12}^{\prime}+m_{22}^{\prime}+j}\left(p_{4}-h_{3}+h_{6}\right)_{m_{3}^{\prime}+m_{11}+m_{13}+m_{22}}}{\left(p_{4}-h_{3}\right)_{2 m_{3}^{\prime}+m_{11}+m_{13}+m_{14}+m_{22}+m_{24}+m_{34}+m_{44}}\left(p_{4}-h_{3}+1-d / 2\right)_{m_{3}^{\prime}}} \\
& \times \frac{\left(p_{2}+h_{3}\right)_{m_{2}^{\prime}-m_{3}^{\prime}+m_{11}^{\prime}}\left(p_{3}-h_{2}+h_{4}\right)_{m_{11}+m_{34}}\left(\bar{p}_{3}+h_{2}+h_{5}\right)_{m_{1}^{\prime}}}{\left(p_{2}+h_{3}+m_{2}^{\prime}\right)_{-m_{3}^{\prime}}\left(\bar{p}_{3}+h_{2}\right)_{2 m_{1}^{\prime}+m_{13}+m_{44}+m_{11}^{\prime}}\left(\bar{p}_{3}+h_{2}+1-d / 2\right)_{m_{1}^{\prime}}} \\
& \times \frac{\left(p_{3}\right)_{m_{1}^{\prime}}\left(p_{2}+h_{2}\right)_{m_{2}^{\prime}+m_{1}^{\prime}+m_{13}+m_{44}+m_{11}^{\prime}}\left(-h_{2}-m_{1}^{\prime}\right)_{m_{1}^{\prime}}\left(-h_{2}\right)_{m_{2}^{\prime}-m_{1}^{\prime}+m_{11}+m_{22}+m_{24}+m_{34}}}{\left(p_{2}\right)_{2 m_{2}^{\prime}+m_{11}+m_{13}+m_{22}+m_{11}^{\prime}+m_{24}+m_{34}+m_{44}}\left(p_{2}+1-d / 2\right)_{m_{2}^{\prime}}} \\
& \times \frac{\left(-h_{3}\right)_{m_{2}^{\prime}+m_{3}^{\prime}+m_{11}+m_{13}+m_{22}+m_{24}+m_{34}+m_{44}}\left(-h_{4}\right)_{m_{22}+m_{24}}\left(-h_{5}\right)_{m_{1}^{\prime}+m_{13}+m_{11}^{\prime}+m_{44}}}{\left(p_{3}-h_{2}\right)_{m_{11}+m_{22}+m_{24}+m_{34}}}
\end{aligned}
$$

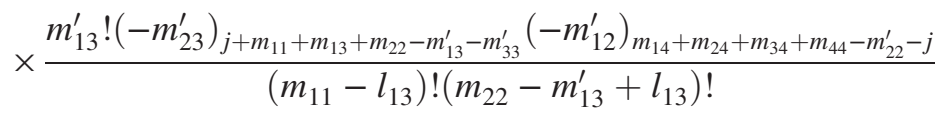

$$
\begin{aligned}
& \times \frac{\left(-h_{3}+h_{6}+m_{2}^{\prime}+m_{11}+m_{13}+m_{22}\right)_{m_{13}^{\prime}+m_{23}^{\prime}+m_{33}^{\prime}-m_{11}-m_{13}-m_{22}-j}}{l_{13} !\left(m_{13}^{\prime}-l_{13}\right) !\left(m_{13}-m_{33}^{\prime}\right) !} \\
& \times \frac{(-1)^{j}}{\left(m_{44}-m_{22}^{\prime}\right) !\left(m_{14}-j\right) ! j ! m_{24} ! m_{34} !} F_{6} \prod_{1 \leq a \leq 3} \frac{\left(u_{a}^{6}\right)^{m_{a}^{\prime}}}{m_{a}^{\prime} !} \prod_{1 \leq a \leq b \leq 3} \frac{\left(1-v_{a b}^{6}\right)^{m_{a b}^{\prime}}}{m_{a b}^{\prime} !} .
\end{aligned}
$$

\footnotetext{
${ }^{10}$ We first change variables such that $k_{13} \rightarrow k_{13}+m_{33}^{\prime}$ and $k_{44} \rightarrow k_{44}+m_{22}^{\prime}$.
} 
We now define $m_{34}=m-m_{24}$ to evaluate the sums over $m_{24}, m_{14}, m$, and finally $m_{44}{ }^{11}$ leading to

$$
\begin{aligned}
& G_{7 \mid \eta_{3} \rightarrow \eta_{4}}=\sum \frac{\left(p_{4}-m_{2}^{\prime}\right)_{m_{3}^{\prime}+j}\left(-h_{6}\right)_{m_{3}^{\prime}+m_{12}^{\prime}+m_{22}^{\prime}+j}\left(p_{4}-h_{3}+h_{6}\right)_{m_{3}^{\prime}+m_{11}+m_{13}+m_{22}}}{\left(p_{4}-h_{3}\right)_{2 m_{3}^{\prime}+m_{12}^{\prime}+m_{22}^{\prime}+m_{11}+m_{13}+m_{22}+j}\left(p_{4}-h_{3}+1-d / 2\right)_{m_{3}^{\prime}}} \\
& \times \frac{\left(p_{2}+h_{3}\right)_{m_{2}^{\prime}-m_{3}^{\prime}+m_{11}^{\prime}}\left(p_{3}-h_{2}+h_{4}\right)_{m_{11}}\left(\bar{p}_{3}+h_{2}+h_{5}\right)_{m_{1}^{\prime}+m_{12}^{\prime}}}{\left(p_{2}+h_{3}+m_{2}^{\prime}\right)_{-m_{3}^{\prime}}\left(\bar{p}_{3}+h_{2}\right)_{2 m_{1}^{\prime}+m_{11}^{\prime}+m_{12}^{\prime}+m_{22}^{\prime}+m_{13}}\left(\bar{p}_{3}+h_{2}+1-d / 2\right)_{m_{1}^{\prime}}} \\
& \times \frac{\left(p_{3}\right)_{m_{1}^{\prime}}\left(p_{2}+h_{2}\right)_{m_{1}^{\prime}+m_{2}^{\prime}+m_{11}^{\prime}+m_{12}^{\prime}+m_{22}^{\prime}+m_{13}}\left(-h_{2}-m_{1}^{\prime}\right)_{m_{1}^{\prime}}\left(-h_{2}\right)_{m_{2}^{\prime}-m_{1}^{\prime}+m_{11}+m_{22}}}{\left(p_{2}\right)_{2 m_{2}^{\prime}+m_{11}^{\prime}+m_{12}^{\prime}+m_{22}^{\prime}+m_{11}+m_{13}+m_{22}}\left(p_{2}+1-d / 2\right)_{m_{2}^{\prime}}} \\
& \times \frac{\left(-h_{3}\right)_{m_{2}^{\prime}+m_{3}^{\prime}+m_{12}^{\prime}+m_{22}^{\prime}+m_{11}+m_{13}+m_{22}}\left(-h_{4}\right)_{m_{22}}\left(-h_{5}\right)_{m_{1}^{\prime}+m_{11}^{\prime}+m_{22}^{\prime}+m_{13}}}{\left(p_{3}-h_{2}\right)_{m_{11}+m_{22}}} \\
& \times \frac{\left(-h_{3}+h_{6}+m_{2}^{\prime}+m_{11}+m_{13}+m_{22}\right)_{m_{13}^{\prime}+m_{23}^{\prime}+m_{33}^{\prime}-m_{11}-m_{13}-m_{22}-j}}{\left(m_{13}^{\prime}-l_{13}\right) !\left(m_{13}-m_{33}^{\prime}\right) !}
\end{aligned}
$$

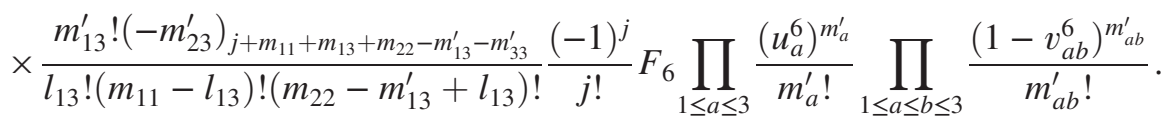

With the help of the first identity involving ${ }_{3} F_{2}$ shown in (A.3), we evaluate the sum over $j$ and get

$$
\begin{aligned}
& G_{7 \mid \eta_{3} \rightarrow \eta_{4}}=\sum \frac{\left(p_{4}-m_{2}^{\prime}\right)_{m_{3}^{\prime}}\left(-h_{6}\right)_{m_{3}^{\prime}+m_{12}^{\prime}+m_{22}^{\prime}}\left(p_{4}-h_{3}+h_{6}\right)_{m_{3}^{\prime}+m_{13}^{\prime}+m_{23}^{\prime}+m_{33}^{\prime}}}{\left(p_{4}-h_{3}\right)_{2 m_{3}^{\prime}+m_{12}^{\prime}+m_{13}^{\prime}+m_{22}^{\prime}+m_{23}^{\prime}+m_{33}^{\prime}}\left(p_{4}-h_{3}+1-d / 2\right)_{m_{3}^{\prime}}} \\
& \times \frac{\left(p_{2}+h_{3}\right)_{m_{2}^{\prime}-m_{3}^{\prime}+m_{11}^{\prime}}\left(p_{3}-h_{2}+h_{4}\right)_{m_{11}}\left(\bar{p}_{3}+h_{2}+h_{5}\right)_{m_{1}^{\prime}+m_{12}^{\prime}}}{\left(p_{2}+h_{3}+m_{2}^{\prime}\right)_{-m_{3}^{\prime}}\left(\bar{p}_{3}+h_{2}\right)_{2 m_{1}^{\prime}+m_{11}^{\prime}+m_{12}^{\prime}+m_{22}^{\prime}+m_{13}}\left(\bar{p}_{3}+h_{2}+1-d / 2\right)_{m_{1}^{\prime}}}
\end{aligned}
$$

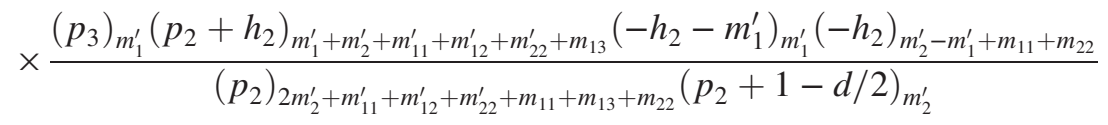

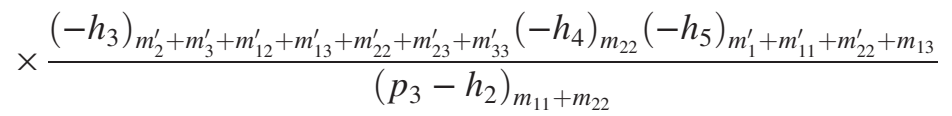

$$
\begin{aligned}
& \times \frac{m_{13}^{\prime} !\left(-m_{23}^{\prime}\right)_{m_{11}+m_{13}+m_{22}-m_{13}^{\prime}-m_{33}^{\prime}}}{l_{13} !\left(m_{13}^{\prime}-l_{13}\right) !\left(m_{13}-m_{33}^{\prime}\right) !\left(m_{11}-l_{13}\right) !\left(m_{22}-m_{13}^{\prime}+l_{13}\right) !} F_{6} \prod_{1 \leq a \leq 3} \frac{\left(u_{a}^{6}\right)^{m_{a}^{\prime}}}{m_{a}^{\prime} !} \prod_{1 \leq a \leq b \leq 3} \frac{\left(1-v_{a b}^{6}\right)^{m_{a b}^{\prime}}}{m_{a b}^{\prime} !} \text {. }
\end{aligned}
$$

We finally change the variables by

$$
m_{11} \rightarrow m_{11}+l_{13}, \quad m_{22} \rightarrow m_{22}+m_{13}^{\prime}-l_{13},
$$

and then define $m_{22}=n-m_{11}$. We can thus evaluate the sums over $m_{11}, n, l_{13}$, and $m_{13}$ after shifting $m_{13} \rightarrow m_{13}+m_{33}^{\prime}$, resulting in

\footnotetext{
${ }^{11}$ We first shift $m_{14} \rightarrow m_{14}+j$ and $m_{44} \rightarrow m_{44}+m_{22}^{\prime}$.
} 


$$
\begin{aligned}
& G_{7 \mid \eta_{3} \rightarrow \eta_{4}}=\sum \frac{\left(p_{4}-m_{2}^{\prime}\right)_{m_{3}^{\prime}}\left(-h_{6}\right)_{m_{3}^{\prime}+m_{12}^{\prime}+m_{22}^{\prime}}\left(p_{4}-h_{3}+h_{6}\right)_{m_{3}^{\prime}+m_{13}^{\prime}+m_{23}^{\prime}+m_{33}^{\prime}}}{\left(p_{4}-h_{3}\right)_{2 m_{3}^{\prime}+m_{12}^{\prime}+m_{13}^{\prime}+m_{22}^{\prime}+m_{23}^{\prime}+m_{33}^{\prime}}\left(p_{4}-h_{3}+1-d / 2\right)_{m_{3}^{\prime}}}
\end{aligned}
$$

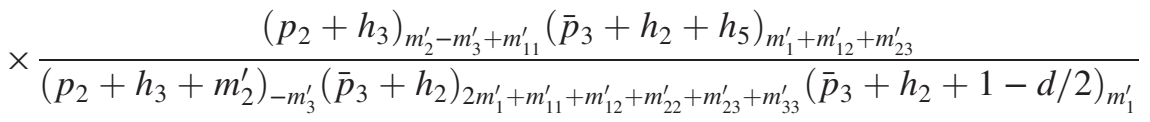

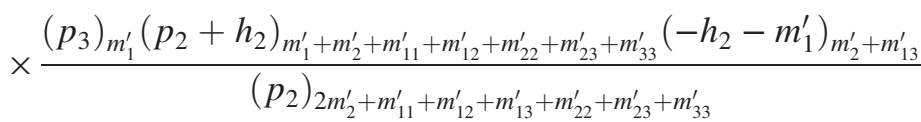

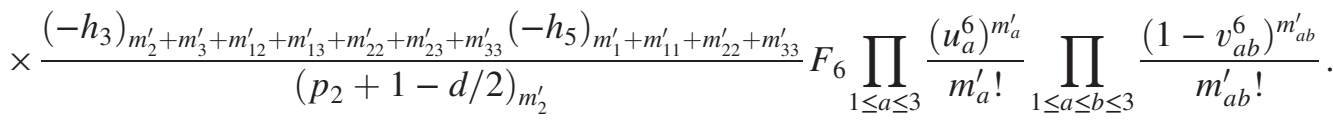

Reexpressing the unprimed variables in terms of the primed variables leads to $G_{6}$ which completes our proof of the OPE limit $\eta_{3} \rightarrow \eta_{4}$ (C.1).

\section{Limit of unit operator}

We focus first on the limit of unit operator given by $\mathcal{O}_{i_{2}}\left(\eta_{2}\right) \rightarrow \mathbb{1}$, for which we have $\Delta_{i_{2}}=0$ as well as $\Delta_{k_{4}}=\Delta_{k_{1}}$. It implies

$$
\left.\left.I_{7\left(\Delta_{k_{1}}, \Delta_{k_{2}}, \Delta_{k_{3}}, \Delta_{k_{4}}\right)}^{\left(\Delta_{i_{i}}, \Delta_{i_{1}}, \Delta_{i}, \Delta_{i_{1}}, \Delta_{i_{6}}, \Delta_{i_{7}}, \Delta_{i_{1}}\right)}\right|_{\substack{\text { extended } \\ \text { snowflake }}} \underset{\mathcal{O}_{i_{2}}\left(\eta_{2}\right) \rightarrow \mathbb{1}}{\longrightarrow} I_{6\left(\Delta_{k_{1}}, \Delta_{k_{2}}, \Delta_{k_{3}}\right)}^{\left(\Delta_{i_{7},}, \Delta_{i_{1}}, \Delta_{i_{1}}, \Delta_{i_{4}}, \Delta_{i_{5}}, \Delta_{i_{6}}\right)}\right|_{\text {snowflake }},
$$

with $p_{4}=p_{2}+h_{3}=0$. Moreover, in this limit the legs and conformal cross-ratios of the six- and seven-point correlation functions are related by

$$
\begin{array}{rlrl}
L_{7} \prod_{1 \leq a \leq 4}\left(u_{a}^{7}\right)^{\frac{\Delta_{\Delta_{a}}}{2}} & =\left(v_{24}^{7}\right)^{-h_{4}}\left(v_{34}^{7}\right)^{h_{4}-h_{2}} L_{6} \prod_{1 \leq a \leq 3}\left(u_{a}^{6}\right)^{\frac{\Delta_{a}}{2}}, \\
u_{1}^{6} & =\frac{u_{1}^{7} u_{4}^{7}}{v_{34}^{7}}, \quad u_{2}^{6}=\frac{u_{2}^{7}}{v_{24}^{7}}, \quad u_{3}^{6}=u_{3}^{7} v_{34}^{7}, \\
v_{11}^{6} & =\frac{v_{11}^{7}}{v_{34}^{7}}, \quad v_{12}^{6}=\frac{v_{12}^{7} v_{34}^{7}}{v_{24}^{7}}, \quad v_{13}^{6}=v_{13}^{7}, \\
v_{22}^{6} & =\frac{v_{22}^{7}}{v_{24}^{7}}, & v_{23}^{6} & =v_{44}^{7}, \quad v_{33}^{6}=\frac{v_{33}^{7} v_{34}^{7}}{v_{24}^{7}} .
\end{array}
$$

These observations lead to the identity $G_{7 \mid \mathcal{O}_{i_{2}} \rightarrow \mathbb{1}}=\left(v_{24}^{7}\right)^{h_{4}}\left(v_{34}^{7}\right)^{h_{2}-h_{4}} G_{6}$ that we now prove.

From the vanishing of $p_{4}$ and $p_{2}+h_{3}$, we have

$$
m_{1}=m_{4}=r, \quad m_{14}=m_{23}=0 .
$$

As a result, we find that 


$$
\begin{aligned}
& G_{7 \mid \mathcal{O}_{i_{2}} \rightarrow \mathbb{1}}=\sum \frac{\left(-h_{6}\right)_{m_{1}+m_{24}+m_{34}+m_{23}^{\prime}}\left(p_{2}+h_{6}\right)_{m_{1}+m_{11}+m_{13}+m_{22}}\left(p_{2}+h_{2}\right)_{m_{1}-m_{2}+m_{3}+m_{13}+m_{23}^{\prime}}}{\left(p_{2}\right)_{2 m_{1}+m_{11}+m_{13}+m_{22}+m_{24}+m_{34}+m_{23}^{\prime}}\left(p_{2}+1-d / 2\right)_{m_{1}}} \\
& \times \frac{\left(p_{3}\right)_{-m_{1}+m_{2}+m_{3}+m_{12}+m_{33}}\left(p_{3}-h_{2}+h_{4}\right)_{m_{2}+m_{11}+m_{34}}\left(\bar{p}_{3}+h_{2}+h_{5}\right)_{m_{3}+m_{12}}}{\left(\bar{p}_{3}+h_{2}\right)_{2 m_{3}+m_{12}+m_{13}+m_{33}+m_{23}^{\prime}}\left(\bar{p}_{3}+h_{2}+1-d / 2\right)_{m_{3}}} \\
& \times \frac{\left(-h_{2}\right)_{m_{1}+m_{2}-m_{3}+m_{11}+m_{22}+m_{24}+m_{34}}\left(-h_{4}\right)_{m_{2}+m_{12}+m_{22}+m_{24}+m_{33}}\left(-h_{5}\right)_{m_{3}+m_{13}+m_{33}+m_{23}^{\prime}}}{\left(p_{3}-h_{2}\right)_{2 m_{2}+m_{11}+m_{12}+m_{22}+m_{24}+m_{33}+m_{34}}\left(p_{3}-h_{2}+1-d / 2\right)_{m_{2}}} \\
& \times \frac{\left(-\bar{p}_{3}-h_{2}+d / 2-m_{3}\right)_{m_{3}}\left(-p_{3}+h_{2}+d / 2-m_{2}\right)_{m_{2}}}{\left(p_{3}\right)_{-m_{1}}} \frac{\left(-m_{2}\right)_{t_{1}}\left(-m_{3}\right)_{t_{2}}}{t_{1} ! t_{2} !} \\
& \times \frac{\left(\bar{p}_{3}-d / 2\right)_{t_{1}+t_{2}}}{\left(p_{3}-m_{1}\right)_{t_{1}+t_{2}}} \frac{\left(p_{3}\right)_{t_{1}+t_{2}}}{\left(\bar{p}_{3}+h_{2}+1-d / 2\right)_{t_{2}}\left(p_{3}-h_{2}+1-d / 2\right)_{t_{1}}} \\
& \times\left(\begin{array}{c}
m_{11} \\
k_{11}
\end{array}\right)\left(\begin{array}{c}
m_{12} \\
k_{12}
\end{array}\right)\left(\begin{array}{c}
m_{22} \\
k_{22}
\end{array}\right)\left(\begin{array}{c}
m_{33} \\
k_{33}
\end{array}\right)\left(\begin{array}{c}
k_{11} \\
m_{11}^{\prime}
\end{array}\right)\left(\begin{array}{c}
k_{12} \\
m_{12}^{\prime}
\end{array}\right)\left(\begin{array}{c}
k_{22} \\
m_{22}^{\prime}
\end{array}\right)\left(\begin{array}{c}
k_{33} \\
m_{33}^{\prime}
\end{array}\right) \\
& \times\left(v_{24}^{7}\right)^{m_{2}+k_{12}+k_{22}+k_{33}}\left(v_{34}^{7}\right)^{m_{1}-m_{3}+k_{11}-k_{12}-k_{33}} \frac{\left(1-v_{24}^{7}\right)^{m_{24}}}{m_{24} !} \frac{\left(1-v_{34}^{7}\right)^{m_{34}}}{m_{34} !} \\
& \times(-1)^{k_{11}+k_{12}+k_{22}+k_{33}+m_{11}^{\prime}+m_{12}^{\prime}+m_{22}^{\prime}+m_{33}^{\prime}} \frac{m_{23} !}{m_{23}^{\prime} !} \prod_{1 \leq a \leq 3} \frac{\left(u_{a}^{6}\right)^{m_{a}}}{m_{a} !} \prod_{1 \leq a \leq b \leq 3} \frac{\left(1-v_{a b}^{6}\right)^{m_{a b}^{\prime}}}{m_{a b} !},
\end{aligned}
$$

where we defined $m_{23}^{\prime}=m_{44}$.

We then shift all of $k_{a b}$ by $k_{a b} \rightarrow k_{a b}+m_{a b}^{\prime}$ and use the fact that

$$
\frac{1}{m_{a b} !}\left(\begin{array}{c}
m_{a b} \\
k_{a b}+m_{a b}^{\prime}
\end{array}\right)\left(\begin{array}{c}
k_{a b}+m_{a b}^{\prime} \\
m_{a b}^{\prime}
\end{array}\right)=\frac{1}{m_{a b}^{\prime} !\left(m_{a b}-m_{a b}^{\prime}\right) !}\left(\begin{array}{c}
m_{a b}-m_{a b}^{\prime} \\
k_{a b}
\end{array}\right)
$$

to write

$$
\begin{aligned}
& G_{7 \mid \mathcal{O}_{i_{2}} \rightarrow \mathbb{1}}=\sum \frac{\left(-h_{6}\right)_{m_{1}+m_{24}+m_{34}+m_{23}^{\prime}}\left(p_{2}+h_{6}\right)_{m_{1}+m_{11}+m_{13}^{\prime}+m_{22}}\left(p_{2}+h_{2}\right)_{m_{1}-m_{2}+m_{3}+m_{13}^{\prime}+m_{23}^{\prime}}}{\left(p_{2}\right)_{2 m_{1}+m_{11}+m_{13}^{\prime}+m_{22}+m_{24}+m_{34}+m_{23}^{\prime}}\left(p_{2}+1-d / 2\right)_{m_{1}}} \\
& \times \frac{\left(p_{3}\right)_{-m_{1}+m_{2}+m_{3}+m_{12}+m_{33}}\left(p_{3}-h_{2}+h_{4}\right)_{m_{2}+m_{11}+m_{34}}\left(\bar{p}_{3}+h_{2}+h_{5}\right)_{m_{3}+m_{12}}}{\left(\bar{p}_{3}+h_{2}\right)_{2 m_{3}+m_{12}+m_{13}^{\prime}+m_{33}+m_{23}^{\prime}}\left(\bar{p}_{3}+h_{2}+1-d / 2\right)_{m_{3}}} \\
& \times \frac{\left(-h_{2}\right)_{m_{1}+m_{2}-m_{3}+m_{11}+m_{22}+m_{24}+m_{34}}\left(-h_{4}\right)_{m_{2}+m_{12}+m_{22}+m_{24}+m_{33}}\left(-h_{5}\right)_{m_{3}+m_{13}^{\prime}+m_{33}+m_{23}^{\prime}}}{\left(p_{3}-h_{2}\right)_{2 m_{2}+m_{11}+m_{12}+m_{22}+m_{24}+m_{33}+m_{34}}\left(p_{3}-h_{2}+1-d / 2\right)_{m_{2}}} \\
& \times \frac{\left(-\bar{p}_{3}-h_{2}+d / 2-m_{3}\right)_{m_{3}}\left(-p_{3}+h_{2}+d / 2-m_{2}\right)_{m_{2}}}{\left(p_{3}\right)_{-m_{1}}} \frac{\left(-m_{2}\right)_{t_{1}}\left(-m_{3}\right)_{t_{2}}}{t_{1} ! t_{2} !} \\
& \times \frac{\left(\bar{p}_{3}-d / 2\right)_{t_{1}+t_{2}}}{\left(p_{3}-m_{1}\right)_{t_{1}+t_{2}}} \frac{\left(p_{3}\right)_{t_{1}+t_{2}}}{\left(\bar{p}_{3}+h_{2}+1-d / 2\right)_{t_{2}}\left(p_{3}-h_{2}+1-d / 2\right)_{t_{1}}} \\
& \times\left(\begin{array}{c}
m_{11}-m_{11}^{\prime} \\
k_{11}
\end{array}\right)\left(\begin{array}{c}
m_{12}-m_{12}^{\prime} \\
k_{12}
\end{array}\right)\left(\begin{array}{c}
m_{22}-m_{22}^{\prime} \\
k_{22}
\end{array}\right)\left(\begin{array}{c}
m_{33}-m_{33}^{\prime} \\
k_{33}
\end{array}\right) \\
& \times \frac{\left(v_{24}^{7}\right)^{m_{2}+k_{12}+k_{22}+k_{33}+m_{12}^{\prime}+m_{22}^{\prime}+m_{33}^{\prime}}\left(v_{34}^{7}\right)^{m_{1}-m_{3}+k_{11}-k_{12}-k_{33}+m_{11}^{\prime}-m_{12}^{\prime}-m_{33}^{\prime}}}{\left(m_{11}-m_{11}^{\prime}\right) !\left(m_{12}-m_{12}^{\prime}\right) !\left(m_{22}-m_{22}^{\prime}\right) !\left(m_{33}-m_{33}^{\prime}\right) !} \\
& \times \frac{\left(1-v_{24}^{7}\right)^{m_{24}}}{m_{24} !} \frac{\left(1-v_{34}^{7}\right)^{m_{34}}}{m_{34} !}(-1)^{k_{11}+k_{12}+k_{22}+k_{33}} \prod_{1 \leq a \leq 3} \frac{\left(u_{a}^{6}\right)^{m_{a}}}{m_{a} !} \prod_{1 \leq a \leq b \leq 3} \frac{\left(1-v_{a b}^{6}\right)^{m_{a b}^{\prime}}}{m_{a b}^{\prime} !},
\end{aligned}
$$

where we defined $m_{13}^{\prime}=m_{13}$. 
Using the identity (A.1), we can compute the sums over $k_{11}$ and $k_{22}$, leading to

$$
\begin{aligned}
& G_{7 \mid \mathcal{O}_{i_{2}} \rightarrow \mathbb{1}}=\sum \frac{\left(-h_{6}\right)_{m_{1}+m_{24}+m_{34}+m_{23}^{\prime}}\left(p_{2}+h_{6}\right)_{m_{1}+m_{11}+m_{13}^{\prime}+m_{22}}\left(p_{2}+h_{2}\right)_{m_{1}-m_{2}+m_{3}+m_{13}^{\prime}+m_{23}^{\prime}}}{\left(p_{2}\right)_{2 m_{1}+m_{11}+m_{13}^{\prime}+m_{22}+m_{24}+m_{34}+m_{23}^{\prime}}\left(p_{2}+1-d / 2\right)_{m_{1}}}
\end{aligned}
$$

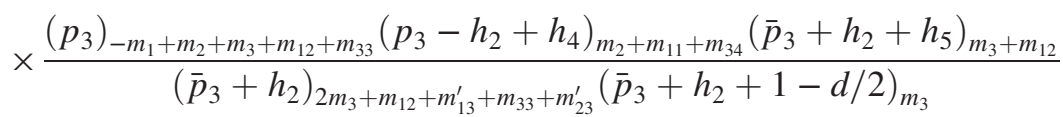

$$
\begin{aligned}
& \times \frac{\left(-h_{2}\right)_{m_{1}+m_{2}-m_{3}+m_{11}+m_{22}+m_{24}+m_{34}}\left(-h_{4}\right)_{m_{2}+m_{12}+m_{22}+m_{24}+m_{33}}\left(-h_{5}\right)_{m_{3}+m_{13}^{\prime}+m_{33}+m_{23}^{\prime}}}{\left(p_{3}-h_{2}\right)_{2 m_{2}+m_{11}+m_{12}+m_{22}+m_{24}+m_{33}+m_{34}}\left(p_{3}-h_{2}+1-d / 2\right)_{m_{2}}} \\
& \times \frac{\left(-\bar{p}_{3}-h_{2}+d / 2-m_{3}\right)_{m_{3}}\left(-p_{3}+h_{2}+d / 2-m_{2}\right)_{m_{2}}}{\left(p_{3}\right)_{-m_{1}}} \frac{\left(-m_{2}\right)_{t_{1}}\left(-m_{3}\right)_{t_{2}}}{t_{1} ! t_{2} !} \\
& \times \frac{\left(\bar{p}_{3}-d / 2\right)_{t_{1}+t_{2}}}{\left(p_{3}-m_{1}\right)_{t_{1}+t_{2}}} \frac{\left(p_{3}\right)_{t_{1}+t_{2}}}{\left(\bar{p}_{3}+h_{2}+1-d / 2\right)_{t_{2}}\left(p_{3}-h_{2}+1-d / 2\right)_{t_{1}}} \\
& \times\left(\begin{array}{c}
m_{12}-m_{12}^{\prime} \\
k_{12}
\end{array}\right)\left(\begin{array}{c}
m_{33}-m_{33}^{\prime} \\
k_{33}
\end{array}\right)\left(\begin{array}{l}
k_{12} \\
r_{12}
\end{array}\right)\left(\begin{array}{l}
k_{33} \\
r_{33}
\end{array}\right) \\
& \times \frac{\left(v_{24}^{7}\right)^{m_{2}+m_{12}^{\prime}+m_{22}^{\prime}+m_{33}^{\prime}}\left(v_{34}^{7}\right)^{m_{1}-m_{3}-k_{12}-k_{33}+m_{11}^{\prime}-m_{12}^{\prime}-m_{33}^{\prime}}}{\left(m_{11}-m_{11}^{\prime}\right) !\left(m_{12}-m_{12}^{\prime}\right) !\left(m_{22}-m_{22}^{\prime}\right) !\left(m_{33}-m_{33}^{\prime}\right) !} \frac{\left(1-v_{24}^{7}\right)^{r_{12}+r_{33}+m_{22}+m_{24}-m_{22}^{\prime}}}{m_{24} !} \\
& \times \frac{\left(1-v_{34}^{7}\right)^{m_{11}+m_{34}-m_{11}^{\prime}}}{m_{34} !}(-1)^{r_{12}+r_{33}+k_{12}+k_{33}} \prod_{1 \leq a \leq 3} \frac{\left(u_{a}^{6}\right)^{m_{a}}}{m_{a} !} \prod_{1 \leq a \leq b \leq 3} \frac{\left(1-v_{a b}^{6}\right)^{m_{a b}^{\prime}}}{m_{a b}^{\prime} !} .
\end{aligned}
$$

We now change $k_{12}$ and $k_{33}$ by $k_{12} \rightarrow k_{12}+r_{12}$ and $k_{33} \rightarrow k_{33}+r_{33}$, respectively, giving us

$$
\begin{aligned}
& G_{7 \mid \mathcal{O}_{i_{2}} \rightarrow \mathbb{1}}=\sum \frac{\left(-h_{6}\right)_{m_{1}+m_{24}+m_{34}+m_{23}^{\prime}}\left(p_{2}+h_{6}\right)_{m_{1}+m_{11}+m_{13}^{\prime}+m_{22}}\left(p_{2}+h_{2}\right)_{m_{1}-m_{2}+m_{3}+m_{13}^{\prime}+m_{23}^{\prime}}}{\left(p_{2}\right)_{2 m_{1}+m_{11}+m_{13}^{\prime}+m_{22}+m_{24}+m_{34}+m_{23}^{\prime}}\left(p_{2}+1-d / 2\right)_{m_{1}}} \\
& \times \frac{\left(p_{3}\right)_{-m_{1}+m_{2}+m_{3}+m_{12}+m_{33}}\left(p_{3}-h_{2}+h_{4}\right)_{m_{2}+m_{11}+m_{34}}\left(\bar{p}_{3}+h_{2}+h_{5}\right)_{m_{3}+m_{12}}}{\left(\bar{p}_{3}+h_{2}\right)_{2 m_{3}+m_{12}+m_{13}^{\prime}+m_{33}+m_{23}^{\prime}}\left(\bar{p}_{3}+h_{2}+1-d / 2\right)_{m_{3}}} \\
& \times \frac{\left(-h_{2}\right)_{m_{1}+m_{2}-m_{3}+m_{11}+m_{22}+m_{24}+m_{34}}\left(-h_{4}\right)_{m_{2}+m_{12}+m_{22}+m_{24}+m_{33}}\left(-h_{5}\right)_{m_{3}+m_{13}^{\prime}+m_{33}+m_{23}^{\prime}}}{\left(p_{3}-h_{2}\right)_{2 m_{2}+m_{11}+m_{12}+m_{22}+m_{24}+m_{33}+m_{34}}\left(p_{3}-h_{2}+1-d / 2\right)_{m_{2}}} \\
& \times \frac{\left(-\bar{p}_{3}-h_{2}+d / 2-m_{3}\right)_{m_{3}}\left(-p_{3}+h_{2}+d / 2-m_{2}\right)_{m_{2}}}{\left(p_{3}\right)_{-m_{1}}} \frac{\left(-m_{2}\right)_{t_{1}}\left(-m_{3}\right)_{t_{2}}}{t_{1} ! t_{2} !} \\
& \times \frac{\left(\bar{p}_{3}-d / 2\right)_{t_{1}+t_{2}}}{\left(p_{3}-m_{1}\right)_{t_{1}+t_{2}}} \frac{\left(p_{3}\right)_{t_{1}+t_{2}}}{\left(\bar{p}_{3}+h_{2}+1-d / 2\right)_{t_{2}}\left(p_{3}-h_{2}+1-d / 2\right)_{t_{1}}} \\
& \times\left(\begin{array}{c}
m_{12}-m_{12}^{\prime}-r_{12} \\
k_{12}
\end{array}\right)\left(\begin{array}{c}
m_{33}-m_{33}^{\prime}-r_{33} \\
k_{33}
\end{array}\right) \\
& \times \frac{\left(v_{24}^{7}\right)^{m_{2}+m_{12}^{\prime}+m_{22}^{\prime}+m_{33}^{\prime}}\left(v_{34}^{7}\right)^{m_{1}-m_{3}-r_{12}-r_{33}-k_{12}-k_{33}+m_{11}^{\prime}-m_{12}^{\prime}-m_{33}^{\prime}}}{r_{12} ! r_{33} !\left(m_{11}-m_{11}^{\prime}\right) !\left(m_{12}-m_{12}^{\prime}-r_{12}\right) !\left(m_{22}-m_{22}^{\prime}\right) !\left(m_{33}-m_{33}^{\prime}-r_{33}\right) !} \\
& \times \frac{\left(1-v_{24}^{7}\right)^{r_{12}+r_{33}+m_{22}+m_{24}-m_{22}^{\prime}}}{m_{24} !} \frac{\left(1-v_{34}^{7}\right)^{m_{11}+m_{34}-m_{11}^{\prime}}}{m_{34} !} \\
& \times(-1)^{k_{12}+k_{33}} \prod_{1 \leq a \leq 3} \frac{\left(u_{a}^{6}\right)^{m_{a}}}{m_{a} !} \prod_{1 \leq a \leq b \leq 3} \frac{\left(1-v_{a b}^{6}\right)^{m_{a b}^{\prime}}}{m_{a b}^{\prime} !} .
\end{aligned}
$$


The sums over $k_{12}$ and $k_{33}$ then lead to

$$
\begin{aligned}
& G_{7 \mid \mathcal{O}_{i_{2}} \rightarrow 1}=\sum \frac{\left(-h_{6}\right)_{m_{1}+m_{24}+m_{34}+m_{23}^{\prime}}\left(p_{2}+h_{6}\right)_{m_{1}+m_{11}+m_{13}^{\prime}+m_{22}}\left(p_{2}+h_{2}\right)_{m_{1}-m_{2}+m_{3}+m_{13}^{\prime}+m_{23}^{\prime}}}{\left(p_{2}\right)_{2 m_{1}+m_{11}+m_{13}^{\prime}+m_{22}+m_{24}+m_{34}+m_{23}^{\prime}}\left(p_{2}+1-d / 2\right)_{m_{1}}} \\
& \times \frac{\left(p_{3}\right)_{-m_{1}+m_{2}+m_{3}+m_{12}+m_{33}}\left(p_{3}-h_{2}+h_{4}\right)_{m_{2}+m_{11}+m_{34}}\left(\bar{p}_{3}+h_{2}+h_{5}\right)_{m_{3}+m_{12}}}{\left(\bar{p}_{3}+h_{2}\right)_{2 m_{3}+m_{12}+m_{13}^{\prime}+m_{33}+m_{23}^{\prime}}\left(\bar{p}_{3}+h_{2}+1-d / 2\right)_{m_{3}}} \\
& \times \frac{\left(-h_{2}\right)_{m_{1}+m_{2}-m_{3}+m_{11}+m_{22}+m_{24}+m_{34}}\left(-h_{4}\right)_{m_{2}+m_{12}+m_{22}+m_{24}+m_{33}}\left(-h_{5}\right)_{m_{3}+m_{13}^{\prime}+m_{33}+m_{23}^{\prime}}}{\left(p_{3}-h_{2}\right)_{2 m_{2}+m_{11}+m_{12}+m_{22}+m_{24}+m_{33}+m_{34}}\left(p_{3}-h_{2}+1-d / 2\right)_{m_{2}}} \\
& \times \frac{\left(-\bar{p}_{3}-h_{2}+d / 2-m_{3}\right)_{m_{3}}\left(-p_{3}+h_{2}+d / 2-m_{2}\right)_{m_{2}}}{\left(p_{3}\right)_{-m_{1}}} \frac{\left(-m_{2}\right)_{t_{1}}\left(-m_{3}\right)_{t_{2}}}{t_{1} ! t_{2} !} \\
& \times \frac{\left(\bar{p}_{3}-d / 2\right)_{t_{1}+t_{2}}}{\left(p_{3}-m_{1}\right)_{t_{1}+t_{2}}} \frac{\left(p_{3}\right)_{t_{1}+t_{2}}}{\left(\bar{p}_{3}+h_{2}+1-d / 2\right)_{t_{2}}\left(p_{3}-h_{2}+1-d / 2\right)_{t_{1}}} \\
& \times \frac{\left(v_{24}^{7}\right)^{m_{2}+m_{12}^{\prime}+m_{22}^{\prime}+m_{33}^{\prime}}\left(v_{34}^{7}\right)^{m_{1}-m_{3}-r_{12}-r_{33}+m_{11}^{\prime}-m_{12}^{\prime}-m_{33}^{\prime}}}{r_{12} ! r_{33} !\left(m_{11}-m_{11}^{\prime}\right) !\left(m_{12}-m_{12}^{\prime}-r_{12}\right) !\left(m_{22}-m_{22}^{\prime}\right) !\left(m_{33}-m_{33}^{\prime}-r_{33}\right) !}
\end{aligned}
$$

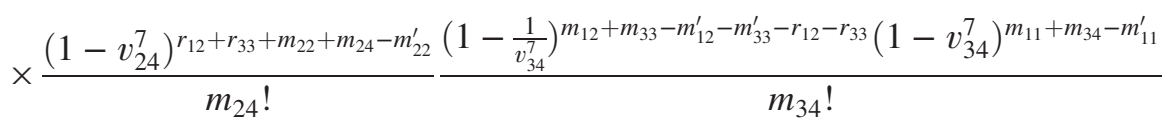

$$
\begin{aligned}
& \times \prod_{1 \leq a \leq 3} \frac{\left(u_{a}^{6}\right)^{m_{a}}}{m_{a} !} \prod_{1 \leq a \leq b \leq 3} \frac{\left(1-v_{a b}^{6}\right)^{m_{a b}^{\prime}}}{m_{a b}^{\prime} !} .
\end{aligned}
$$

Changing variables such that

$$
m_{11} \rightarrow m_{11}+m_{11}^{\prime}, \quad m_{12} \rightarrow m_{12}+m_{11}^{\prime}+r_{12}, \quad m_{22} \rightarrow m_{22}+m_{22}^{\prime}, \quad m_{33} \rightarrow m_{33}+m_{33}^{\prime}+r_{33},
$$

we obtain

$$
\begin{aligned}
& G_{7 \mid \mathcal{O}_{i_{2}} \rightarrow \mathbb{1}}=\sum \frac{\left(-h_{6}\right)_{m_{1}+m_{24}+m_{34}+m_{23}^{\prime}}\left(p_{2}+h_{6}\right)_{m_{1}+m_{11}+m_{11}^{\prime}+m_{13}^{\prime}+m_{22}+m_{22}^{\prime}}\left(p_{2}+h_{2}\right)_{m_{1}-m_{2}+m_{3}+m_{13}^{\prime}+m_{23}^{\prime}}}{\left(p_{2}\right)_{2 m_{1}+m_{11}+m_{11}^{\prime}+m_{13}^{\prime}+m_{22}+m_{22}^{\prime}+m_{24}+m_{34}+m_{23}^{\prime}}\left(p_{2}+1-d / 2\right)_{m_{1}}}
\end{aligned}
$$

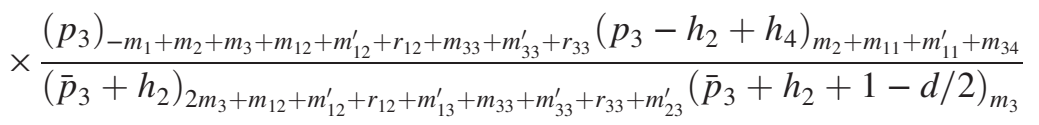

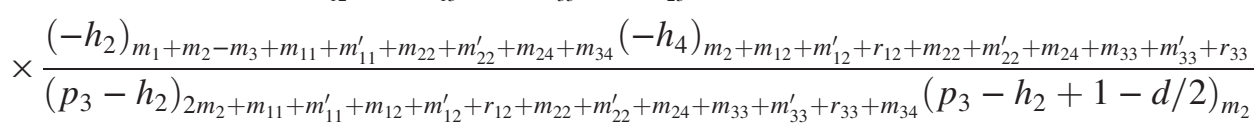

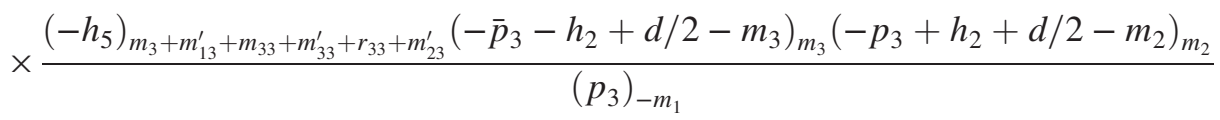

$$
\begin{aligned}
& \times \frac{\left(-m_{2}\right)_{t_{1}}\left(-m_{3}\right)_{t_{2}}}{t_{1} ! t_{2} !} \frac{\left(\bar{p}_{3}-d / 2\right)_{t_{1}+t_{2}}}{\left(p_{3}-m_{1}\right)_{t_{1}+t_{2}}} \frac{\left(p_{3}\right)_{t_{1}+t_{2}}\left(\bar{p}_{3}+h_{2}+h_{5}\right)_{m_{3}+m_{12}+m_{12}^{\prime}+r_{12}}}{\left(\bar{p}_{3}+h_{2}+1-d / 2\right)_{t_{2}}\left(p_{3}-h_{2}+1-d / 2\right)_{t_{1}}} \\
& \times \frac{\left(v_{24}^{7}\right)^{m_{2}+m_{12}^{\prime}+m_{22}^{\prime}+m_{33}^{\prime}}\left(v_{34}^{7}\right)^{m_{1}-m_{3}-r_{12}-r_{33}+m_{11}^{\prime}-m_{12}^{\prime}-m_{33}^{\prime}}}{r_{12} ! r_{33} ! m_{11} ! m_{12} ! m_{22} ! m_{33} !} \\
& \times \frac{\left(1-v_{24}^{7}\right)^{r_{12}+r_{33}+m_{22}+m_{24}}}{m_{24} !} \frac{\left(1-\frac{1}{v_{34}^{7}}\right)^{m_{12}+m_{33}}\left(1-v_{34}^{7}\right)^{m_{11}+m_{34}}}{m_{34} !} \prod_{1 \leq a \leq 3} \frac{\left(u_{a}^{6}\right)^{m_{a}}}{m_{a} !} \prod_{1 \leq a \leq b \leq 3} \frac{\left(1-v_{a b}^{6}\right)^{m_{a b}^{\prime}}}{m_{a b}^{\prime} !} .
\end{aligned}
$$

We now define

$$
m_{24}=n_{24}-m_{22}-r_{12}-r_{33}, \quad m_{34}=n_{34}-m_{11}, \quad m_{33}=s_{34}-m_{12},
$$


such that

$$
\begin{aligned}
& G_{7 \mid \mathcal{O}_{i_{2}} \rightarrow \mathbb{1}}=\sum \frac{\left(-h_{6}\right)_{m_{1}+n_{24}+n_{34}+m_{23}^{\prime}-m_{11}-m_{22}-r_{12}-r_{33}}\left(p_{2}+h_{6}\right)_{m_{1}+m_{11}+m_{11}^{\prime}+m_{13}^{\prime}+m_{22}+m_{22}^{\prime}}}{\left(p_{2}\right)_{2 m_{1}+m_{11}^{\prime}+m_{13}^{\prime}+m_{22}^{\prime}+n_{24}+n_{34}-r_{12}-r_{33}+m_{23}^{\prime}}\left(p_{2}+1-d / 2\right)_{m_{1}}}
\end{aligned}
$$

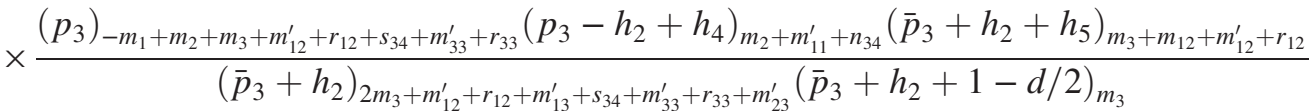

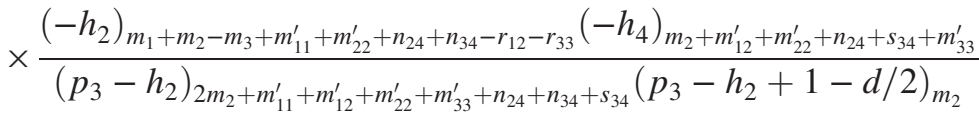

$$
\begin{aligned}
& \times \frac{\left(-h_{5}\right)_{m_{3}+m_{13}^{\prime}+s_{34}-m_{12}+m_{33}^{\prime}+r_{33}+m_{23}^{\prime}}\left(-\bar{p}_{3}-h_{2}+d / 2-m_{3}\right)_{m_{3}}\left(-p_{3}+h_{2}+d / 2-m_{2}\right)_{m_{2}}}{\left(p_{3}\right)_{-m_{1}}} \\
& \times \frac{\left(-m_{2}\right)_{t_{1}}\left(-m_{3}\right)_{t_{2}}}{t_{1} ! t_{2} !} \frac{\left(\bar{p}_{3}-d / 2\right)_{t_{1}+t_{2}}}{\left(p_{3}-m_{1}\right)_{t_{1}+t_{2}}} \frac{\left(p_{2}+h_{2}\right)_{m_{1}-m_{2}+m_{3}+m_{13}^{\prime}+m_{23}^{\prime}}\left(p_{3}\right)_{t_{1}+t_{2}}}{\left(\bar{p}_{3}+h_{2}+1-d / 2\right)_{t_{2}}\left(p_{3}-h_{2}+1-d / 2\right)_{t_{1}}} \\
& \times \frac{\left(v_{24}^{7}\right)^{m_{2}+m_{12}^{\prime}+m_{22}^{\prime}+m_{33}^{\prime}}\left(v_{34}^{7}\right)^{m_{1}-m_{3}-r_{12}-r_{33}+m_{11}^{\prime}-m_{12}^{\prime}-m_{33}^{\prime}}}{r_{12} ! r_{33} ! m_{11} ! m_{12} ! m_{22} !\left(s_{34}-m_{12}\right) !} \\
& \times \frac{\left(1-v_{24}^{7}\right)^{n_{24}}}{\left(n_{24}-m_{22}-r_{12}-r_{33}\right) !} \frac{\left(1-\frac{1}{v_{34}^{7}}\right)^{s_{34}}\left(1-v_{34}^{7}\right)^{n_{34}}}{\left(n_{34}-m_{11}\right) !} \prod_{1 \leq a \leq 3} \frac{\left(u_{a}^{6}\right)^{m_{a}}}{m_{a} !} \prod_{1 \leq a \leq b \leq 3} \frac{\left(1-v_{a b}^{6}\right)^{m_{a b}^{\prime}}}{m_{a b}^{\prime} !} .
\end{aligned}
$$

The sums over $m_{11}, m_{12}$, and $m_{22}$ can be performed, leading to

$$
\begin{aligned}
& G_{7 \mid \mathcal{O}_{i_{2}} \rightarrow \mathbb{1}}=\sum \frac{\left(-h_{6}\right)_{m_{1}+m_{23}^{\prime}}\left(p_{2}+h_{6}\right)_{m_{1}+m_{11}^{\prime}+m_{13}^{\prime}+m_{22}^{\prime}}\left(p_{2}+h_{2}\right)_{m_{1}-m_{2}+m_{3}+m_{13}^{\prime}+m_{23}^{\prime}}}{\left(p_{2}\right)_{2 m_{1}+m_{11}^{\prime}+m_{13}^{\prime}+m_{22}^{\prime}+m_{23}^{\prime}}\left(p_{2}+1-d / 2\right)_{m_{1}}}
\end{aligned}
$$

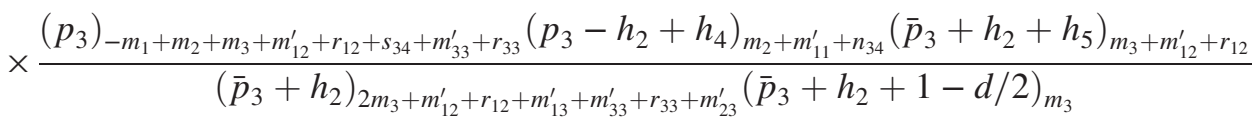

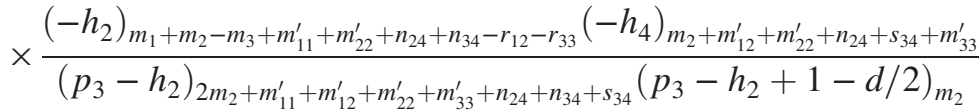

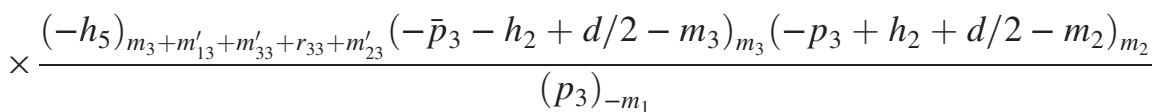

$$
\begin{aligned}
& \times \frac{\left(-m_{2}\right)_{t_{1}}\left(-m_{3}\right)_{t_{2}}}{t_{1} ! t_{2} !} \frac{\left(\bar{p}_{3}-d / 2\right)_{t_{1}+t_{2}}}{\left(p_{3}-m_{1}\right)_{t_{1}+t_{2}}} \frac{\left(p_{3}\right)_{t_{1}+t_{2}}}{\left(\bar{p}_{3}+h_{2}+1-d / 2\right)_{t_{2}}\left(p_{3}-h_{2}+1-d / 2\right)_{t_{1}}}
\end{aligned}
$$

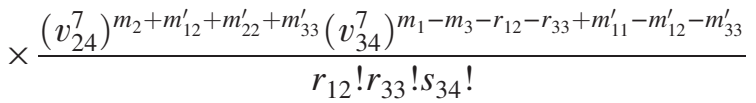

$$
\begin{aligned}
& \times \frac{\left(1-v_{24}^{7}\right)^{n_{24}}}{\left(n_{24}-r_{12}-r_{33}\right) !} \frac{\left(1-\frac{1}{v_{34}^{7}}\right)^{s_{34}}\left(1-v_{34}^{7}\right)^{n_{34}}}{n_{34} !} \prod_{1 \leq a 3} \frac{\left(u_{a}^{6}\right)^{m_{a}}}{m_{a} !} \prod_{1 \leq a \leq b \leq 3} \frac{\left(1-v_{a b}^{6}\right)^{m_{a b}^{\prime}}}{m_{a b}^{\prime} !} .
\end{aligned}
$$


We now redefine $r_{33}=r-r_{12}$ to evaluate the sum over $r_{12}$ and get

$$
\begin{aligned}
& G_{7 \mid \mathcal{O}_{i_{2}} \rightarrow \mathbb{1}}=\sum \frac{\left(-h_{6}\right)_{m_{1}+m_{23}^{\prime}}\left(p_{2}+h_{6}\right)_{m_{1}+m_{11}^{\prime}+m_{13}^{\prime}+m_{22}^{\prime}}\left(p_{2}+h_{2}\right)_{m_{1}-m_{2}+m_{3}+m_{13}^{\prime}+m_{23}^{\prime}}}{\left(p_{2}\right)_{2 m_{1}+m_{11}^{\prime}+m_{13}^{\prime}+m_{22}^{\prime}+m_{23}^{\prime}}\left(p_{2}+1-d / 2\right)_{m_{1}}} \\
& \times \frac{\left(p_{3}\right)_{-m_{1}+m_{2}+m_{3}+m_{12}^{\prime}+s_{34}+m_{33}^{\prime}+r}\left(p_{3}-h_{2}+h_{4}\right)_{m_{2}+m_{11}^{\prime}+n_{34}}\left(\bar{p}_{3}+h_{2}+h_{5}\right)_{m_{3}+m_{12}^{\prime}}}{\left(\bar{p}_{3}+h_{2}\right)_{2 m_{3}+m_{12}^{\prime}+m_{13}^{\prime}+m_{23}^{\prime}+m_{33}^{\prime}}\left(\bar{p}_{3}+h_{2}+1-d / 2\right)_{m_{3}}}
\end{aligned}
$$

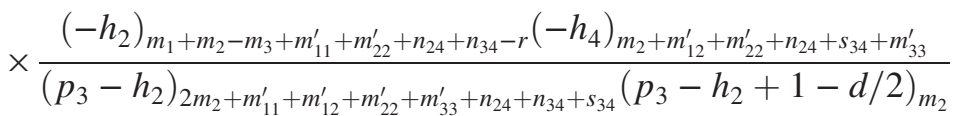

$$
\begin{aligned}
& \times \frac{\left(-h_{5}\right)_{m_{3}+m_{13}^{\prime}+m_{23}^{\prime}+m_{33}^{\prime}}\left(-\bar{p}_{3}-h_{2}+d / 2-m_{3}\right)_{m_{3}}\left(-p_{3}+h_{2}+d / 2-m_{2}\right)_{m_{2}}}{\left(p_{3}\right)_{-m_{1}}} \\
& \times \frac{\left(-m_{2}\right)_{t_{1}}\left(-m_{3}\right)_{t_{2}}}{t_{1} ! t_{2} !} \frac{\left(\bar{p}_{3}-d / 2\right)_{t_{1}+t_{2}}}{\left(p_{3}-m_{1}\right)_{t_{1}+t_{2}}} \frac{\left(p_{3}\right)_{t_{1}+t_{2}}}{\left(\bar{p}_{3}+h_{2}+1-d / 2\right)_{t_{2}}\left(p_{3}-h_{2}+1-d / 2\right)_{t_{1}}}
\end{aligned}
$$

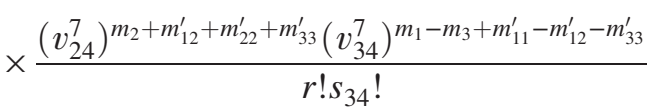

$$
\begin{aligned}
& \times\left(\begin{array}{l}
r \\
j
\end{array}\right) \frac{\left(1-v_{24}^{7}\right)^{n_{24}}}{\left(n_{24}-r\right) !} \frac{\left(1-\frac{1}{v_{34}^{7}}\right)^{j+s_{34}}\left(1-v_{34}^{7}\right)^{n_{34}}}{n_{34} !}(-1)^{j} \prod_{1 \leq a \leq 3} \frac{\left(u_{a}^{6}\right)^{m_{a}}}{m_{a} !} \prod_{1 \leq a \leq b \leq 3} \frac{\left(1-v_{a b}^{6}\right)^{m_{a b}^{\prime}}}{m_{a b}^{\prime} !},
\end{aligned}
$$

where we also expanded $\left(v_{34}^{7}\right)^{-r}$ in a power series in $1-v_{34}^{7}$.

After shifting $r$ by $r \rightarrow r+j$, we evaluate the sum over $r$, leading to

$$
\begin{aligned}
& G_{7 \mid \mathcal{O}_{i_{2}} \rightarrow \mathbb{1}}=\sum \frac{\left(-h_{6}\right)_{m_{1}+m_{23}^{\prime}}\left(p_{2}+h_{6}\right)_{m_{1}+m_{11}^{\prime}+m_{13}^{\prime}+m_{22}^{\prime}}\left(p_{2}+h_{2}\right)_{m_{1}-m_{2}+m_{3}+m_{13}^{\prime}+m_{23}^{\prime}}}{\left(p_{2}\right)_{2 m_{1}+m_{11}^{\prime}+m_{13}^{\prime}+m_{22}^{\prime}+m_{23}^{\prime}}\left(p_{2}+1-d / 2\right)_{m_{1}}} \\
& \times \frac{\left(p_{3}\right)_{-m_{1}+m_{2}+m_{3}+m_{12}^{\prime}+s_{34}+m_{33}^{\prime}+j}\left(p_{3}-h_{2}+h_{4}\right)_{m_{2}+m_{11}^{\prime}+n_{34}}\left(\bar{p}_{3}+h_{2}+h_{5}\right)_{m_{3}+m_{12}^{\prime}}}{\left(\bar{p}_{3}+h_{2}\right)_{2 m_{3}+m_{12}^{\prime}+m_{13}^{\prime}+m_{23}^{\prime}+m_{33}^{\prime}}\left(\bar{p}_{3}+h_{2}+1-d / 2\right)_{m_{3}}}
\end{aligned}
$$

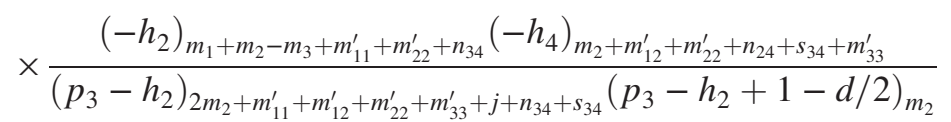

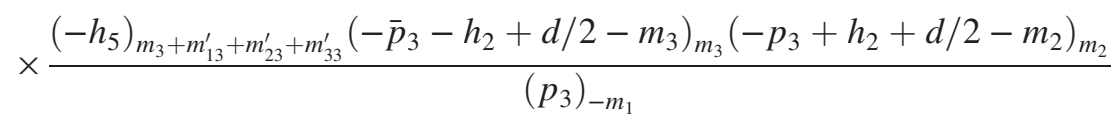

$$
\begin{aligned}
& \times \frac{\left(-m_{2}\right)_{t_{1}}\left(-m_{3}\right)_{t_{2}}}{t_{1} ! t_{2} !} \frac{\left(\bar{p}_{3}-d / 2\right)_{t_{1}+t_{2}}}{\left(p_{3}-m_{1}\right)_{t_{1}+t_{2}}} \frac{\left(p_{3}\right)_{t_{1}+t_{2}}}{\left(\bar{p}_{3}+h_{2}+1-d / 2\right)_{t_{2}}\left(p_{3}-h_{2}+1-d / 2\right)_{t_{1}}}
\end{aligned}
$$

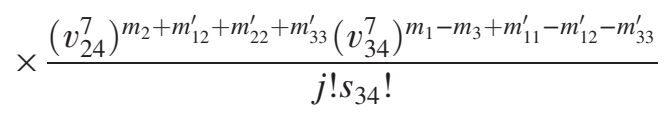

$$
\begin{aligned}
& \times \frac{\left(1-v_{24}^{7}\right)^{n_{24}}}{\left(n_{24}-j\right) !} \frac{\left(1-\frac{1}{v_{34}^{7}}\right)^{j+s_{34}}\left(1-v_{34}^{7}\right)^{n_{34}}}{n_{34} !}(-1)^{j} \prod_{1 \leq a \leq 3} \frac{\left(u_{a}^{6}\right)^{m_{a}}}{m_{a} !} \prod_{1 \leq a \leq b \leq 3} \frac{\left(1-v_{a b}^{6}\right)^{m_{a b}^{\prime}}}{m_{a b}^{\prime} !} .
\end{aligned}
$$


Changing $n_{24}$ by $n_{24}+j$, the sum over $n_{24}$ gives

$$
\begin{aligned}
& G_{7 \mid \mathcal{O}_{i_{2}} \rightarrow \mathbb{1}}=\sum \frac{\left(-h_{6}\right)_{m_{1}+m_{23}^{\prime}}\left(p_{2}+h_{6}\right)_{m_{1}+m_{11}^{\prime}+m_{13}^{\prime}+m_{22}^{\prime}}\left(p_{2}+h_{2}\right)_{m_{1}-m_{2}+m_{3}+m_{13}^{\prime}+m_{23}^{\prime}}}{\left(p_{2}\right)_{2 m_{1}+m_{11}^{\prime}+m_{13}^{\prime}+m_{22}^{\prime}+m_{23}^{\prime}}\left(p_{2}+1-d / 2\right)_{m_{1}}} \\
& \times \frac{\left(p_{3}\right)_{-m_{1}+m_{2}+m_{3}+m_{12}^{\prime}+s_{34}+m_{33}^{\prime}+j}\left(p_{3}-h_{2}+h_{4}\right)_{m_{2}+m_{11}^{\prime}+n_{34}}\left(\bar{p}_{3}+h_{2}+h_{5}\right)_{m_{3}+m_{12}^{\prime}}}{\left(\bar{p}_{3}+h_{2}\right)_{2 m_{3}+m_{12}^{\prime}+m_{13}^{\prime}+m_{23}^{\prime}+m_{33}^{\prime}}\left(\bar{p}_{3}+h_{2}+1-d / 2\right)_{m_{3}}}
\end{aligned}
$$

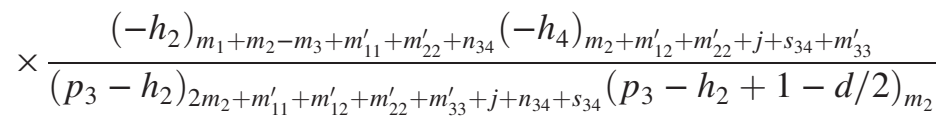

$$
\begin{aligned}
& \times \frac{\left(-h_{5}\right)_{m_{3}+m_{13}^{\prime}+m_{23}^{\prime}+m_{33}^{\prime}}\left(-\bar{p}_{3}-h_{2}+d / 2-m_{3}\right)_{m_{3}}\left(-p_{3}+h_{2}+d / 2-m_{2}\right)_{m_{2}}}{\left(p_{3}\right)_{-m_{1}}} \\
& \times \frac{\left(-m_{2}\right)_{t_{1}}\left(-m_{3}\right)_{t_{2}}}{t_{1} ! t_{2} !} \frac{\left(\bar{p}_{3}-d / 2\right)_{t_{1}+t_{2}}}{\left(p_{3}-m_{1}\right)_{t_{1}+t_{2}}} \frac{\left(p_{3}\right)_{t_{1}+t_{2}}}{\left(\bar{p}_{3}+h_{2}+1-d / 2\right)_{t_{2}}\left(p_{3}-h_{2}+1-d / 2\right)_{t_{1}}} \\
& \times \frac{\left(v_{24}^{7}\right)^{h_{4}-j-s_{34}}\left(v_{34}^{7}\right)^{m_{1}-m_{3}+m_{11}^{\prime}-m_{12}^{\prime}-m_{33}^{\prime}}}{s_{34} !} \\
& \times \frac{\left(1-v_{24}^{7}\right)^{j}}{j !} \frac{\left(1-\frac{1}{v_{34}^{7}}\right)^{j+s_{34}}\left(1-v_{34}^{7}\right)^{n_{34}}}{n_{34} !}(-1)^{j} \prod_{1 \leq a 3} \frac{\left(u_{a}^{6}\right)^{m_{a}}}{m_{a} !} \prod_{1 \leq a \leq b \leq 3} \frac{\left(1-v_{a b}^{6}\right)^{m_{a b}^{\prime}}}{m_{a b}^{\prime} !} .
\end{aligned}
$$

We now define $s_{34}=s-j$ and evaluate the sum over $j$ to reach

$$
\begin{aligned}
& G_{7 \mid \mathcal{O}_{i_{2}} \rightarrow \mathbb{1}}=\sum \frac{\left(-h_{6}\right)_{m_{1}+m_{23}^{\prime}}\left(p_{2}+h_{6}\right)_{m_{1}+m_{11}^{\prime}+m_{13}^{\prime}+m_{22}^{\prime}}\left(p_{2}+h_{2}\right)_{m_{1}-m_{2}+m_{3}+m_{13}^{\prime}+m_{23}^{\prime}}}{\left(p_{2}\right)_{2 m_{1}+m_{11}^{\prime}+m_{13}^{\prime}+m_{22}^{\prime}+m_{23}^{\prime}}\left(p_{2}+1-d / 2\right)_{m_{1}}}
\end{aligned}
$$

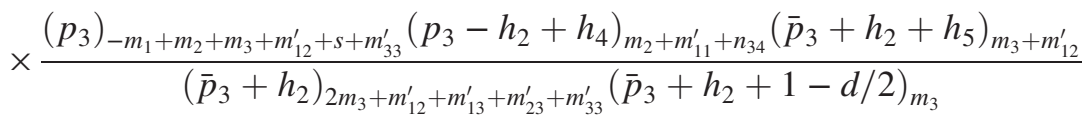

$$
\begin{aligned}
& \times \frac{\left(-h_{2}\right)_{m_{1}+m_{2}-m_{3}+m_{11}^{\prime}+m_{22}^{\prime}+n_{34}}\left(-h_{4}\right)_{m_{2}+m_{12}^{\prime}+m_{22}^{\prime}+s+m_{33}^{\prime}}}{\left(p_{3}-h_{2}\right)_{2 m_{2}+m_{11}^{\prime}+m_{12}^{\prime}+m_{22}^{\prime}+m_{33}^{\prime}+n_{34}+s}\left(p_{3}-h_{2}+1-d / 2\right)_{m_{2}}} \\
& \times \frac{\left(-h_{5}\right)_{m_{3}+m_{13}^{\prime}+m_{23}^{\prime}+m_{33}^{\prime}}\left(-\bar{p}_{3}-h_{2}+d / 2-m_{3}\right)_{m_{3}}\left(-p_{3}+h_{2}+d / 2-m_{2}\right)_{m_{2}}}{\left(p_{3}\right)_{-m_{1}}} \frac{\left(-m_{2}\right)_{t_{1}}\left(-m_{3}\right)_{t_{2}}}{t_{1} ! t_{2} !} \\
& \times \frac{\left(\bar{p}_{3}-d / 2\right)_{t_{1}+t_{2}}}{\left(p_{3}-m_{1}\right)_{t_{1}+t_{2}}} \frac{\left(p_{3}\right)_{t_{1}+t_{2}}}{\left(\bar{p}_{3}+h_{2}+1-d / 2\right)_{t_{2}}\left(p_{3}-h_{2}+1-d / 2\right)_{t_{1}}} \\
& \times \frac{\left(v_{24}^{7}\right)^{h_{4}}\left(v_{34}^{7}\right)^{m_{1}-m_{3}+m_{11}^{\prime}-m_{12}^{\prime}-m_{33}^{\prime}}}{s !} \frac{\left(1-\frac{1}{v_{34}^{7}}\right)^{s}\left(1-v_{34}^{7}\right)^{n_{34}}}{n_{34} !} \prod_{1 \leq a \leq 3} \frac{\left(u_{a}^{6}\right)^{m_{a}}}{m_{a} !} \prod_{1 \leq a \leq b \leq 3} \frac{\left(1-v_{a b}^{6}\right)^{m_{a b}^{\prime}}}{m_{a b}^{\prime} !} \text {. }
\end{aligned}
$$

We then express the sum over $s$ in terms of a hypergeometric function and use the first identity in (A.2) to rewrite the summation over $s$, leading to 


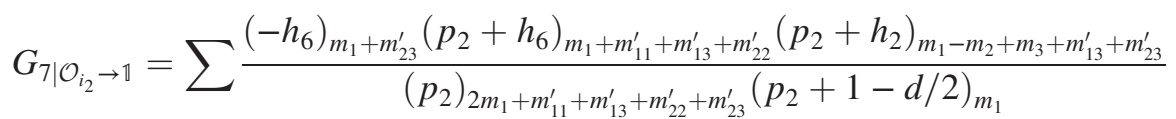

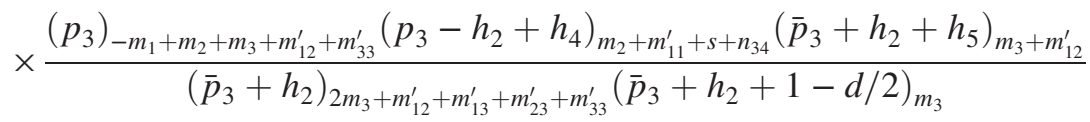

$$
\begin{aligned}
& \times \frac{\left(-h_{2}\right)_{m_{1}+m_{2}-m_{3}+m_{11}^{\prime}+m_{22}^{\prime}+s+n_{34}}\left(-h_{4}\right)_{m_{2}+m_{12}^{\prime}+m_{22}^{\prime}+m_{33}^{\prime}}}{\left(p_{3}-h_{2}\right)_{2 m_{2}+m_{11}^{\prime}+m_{12}^{\prime}+m_{22}^{\prime}+m_{33}^{\prime}+n_{34}+s}\left(p_{3}-h_{2}+1-d / 2\right)_{m_{2}}}
\end{aligned}
$$

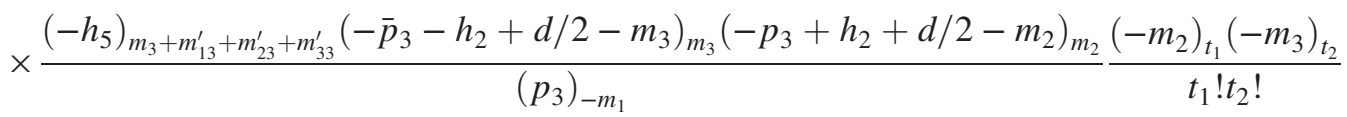

$$
\begin{aligned}
& \times \frac{\left(\bar{p}_{3}-d / 2\right)_{t_{1}+t_{2}}}{\left(p_{3}-m_{1}\right)_{t_{1}+t_{2}}} \frac{\left(p_{3}\right)_{t_{1}+t_{2}}}{\left(\bar{p}_{3}+h_{2}+1-d / 2\right)_{t_{2}}\left(p_{3}-h_{2}+1-d / 2\right)_{t_{1}}} \\
& \times(-1)^{s} \frac{\left(v_{24}^{7}\right)^{h_{4}}\left(v_{34}^{7}\right)^{h_{2}-h_{4}-s-n_{34}}}{s !} \frac{\left(1-v_{34}^{7}\right)^{s+n_{34}}}{n_{34} !} \prod_{1 \leq a \leq 3} \frac{\left(u_{a}^{6}\right)^{m_{a}}}{m_{a} !} \prod_{1 \leq a \leq b \leq 3} \frac{\left(1-v_{a b}^{6}\right)^{m_{a b}^{\prime}}}{m_{a b}^{\prime} !} \text {, }
\end{aligned}
$$

where we used $\left(1-1 / v_{34}^{7}\right)^{s}=(-1)^{s}\left(v_{34}^{7}\right)^{-s}\left(1-v_{34}^{7}\right)^{s}$.

After defining $n_{34}=m-s$, we evaluate the sum over $s$ to get

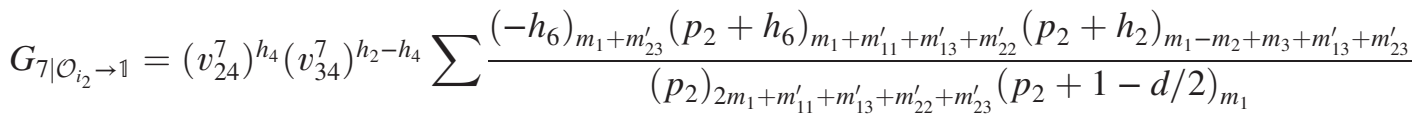

$$
\begin{aligned}
& \times \frac{\left(p_{3}\right)_{-m_{1}+m_{2}+m_{3}+m_{12}^{\prime}+m_{33}^{\prime}}\left(p_{3}-h_{2}+h_{4}\right)_{m_{2}+m_{11}^{\prime}}\left(\bar{p}_{3}+h_{2}+h_{5}\right)_{m_{3}+m_{12}^{\prime}}}{\left(\bar{p}_{3}+h_{2}\right)_{2 m_{3}+m_{12}^{\prime}+m_{13}^{\prime}+m_{23}^{\prime}+m_{33}^{\prime}}\left(\bar{p}_{3}+h_{2}+1-d / 2\right)_{m_{3}}}
\end{aligned}
$$

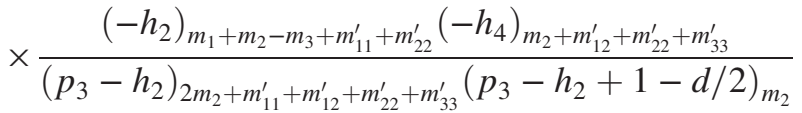

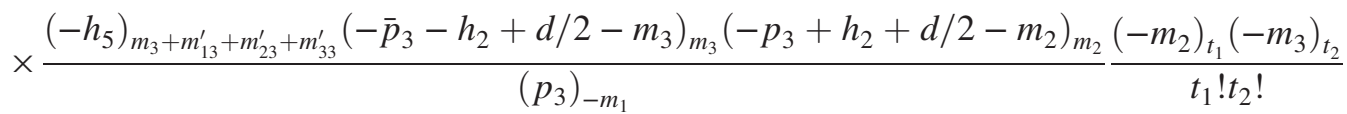

$$
\begin{aligned}
& \times \frac{\left(\bar{p}_{3}-d / 2\right)_{t_{1}+t_{2}}}{\left(p_{3}-m_{1}\right)_{t_{1}+t_{2}}} \frac{\left(p_{3}\right)_{t_{1}+t_{2}}}{\left(\bar{p}_{3}+h_{2}+1-d / 2\right)_{t_{2}}\left(p_{3}-h_{2}+1-d / 2\right)_{t_{1}}} \prod_{1 \leq a \leq 3} \frac{\left(u_{a}^{6}\right)^{m_{a}}}{m_{a} !} \prod_{1 \leq a \leq b \leq 3} \frac{\left(1-v_{a b}^{6}\right)^{m_{a b}^{\prime}}}{m_{a b}^{\prime} !} \\
& =\left(v_{24}^{7}\right)^{h_{4}}\left(v_{34}^{7}\right)^{h_{2}-h_{4}} G_{6} \text {, }
\end{aligned}
$$

which complete our proof for the limit of unit operator $\mathcal{O}_{2}\left(\eta_{2}\right) \rightarrow \mathbb{1}$ (C.2).

The last limit of unit operator to verify is $\mathcal{O}_{i_{3}}\left(\eta_{3}\right)=\mathbb{1}$ which leads to

$$
\left.\left.I_{7\left(\Delta_{k_{1}}, \Delta_{k_{2}}, \Delta_{k_{3}}, \Delta_{k_{4}}\right)}^{\left(\Delta_{i_{2}}, \Delta_{i_{3}}, \Delta_{i_{i}}, \Delta_{i_{2}}, \Delta_{i_{6}}, \Delta_{i_{7}}, \Delta_{i_{1}}\right)}\right|_{\substack{\text { extended } \\ \text { snowflake }}} \underset{\mathcal{O}_{i_{2}}\left(\eta_{2}\right) \rightarrow \mathbb{1}}{\longrightarrow} I_{6\left(\Delta_{k_{3}}, \Delta_{k_{1}}, \Delta_{k_{4}}\right)}^{\left(\Delta_{i_{5}}, \Delta_{i_{6}}, \Delta_{i_{i}}, \Delta_{i_{2}}, \Delta_{i_{7}}, \Delta_{i_{1}}\right)}\right|_{\text {comb }}
$$

Moreover, in this limit we have $\Delta_{i_{3}}=0, \Delta_{k_{2}}=\Delta_{i_{4}}$ as well as

$$
\begin{array}{rlrl}
L_{7} \prod_{1 \leq a \leq 4}\left(u_{a}^{7}\right)^{\frac{\Delta_{\Delta_{k}}}{2}} & =\left(v_{23}^{6}\right)^{h_{3}-h_{6}}\left(v_{12}^{6}\right)^{h_{6}} L_{6} \prod_{1 \leq a \leq 3}\left(u_{i}^{6}\right)^{\frac{\Delta_{k_{a}^{\prime}}}{2}}, \\
u_{1}^{6} & =u_{3}^{7}, \quad u_{2}^{6}=\frac{u_{1}^{7} v_{14}^{7}}{v_{34}^{7}}, \quad u_{3}^{6}=\frac{u_{4}^{7}}{v_{34}^{7}}, \\
v_{11}^{6} & =v_{23}^{7}, & v_{12}^{6}=\frac{1}{v_{34}^{7}}, & v_{13}^{6}=\frac{v_{11}^{7} v_{14}^{7}}{v_{34}^{7}}, \\
v_{22}^{6} & =\frac{v_{44}^{7}}{v_{34}^{7}}, & v_{23}^{6} & =\frac{v_{14}^{7}}{v_{34}^{7}},
\end{array}
$$


for the legs and conformal cross-ratios. Here, the primed parameters correspond to the parameters relevant for the rhs of (C.3). Considering that $h_{4}=p_{5}=p_{2}+h_{3}=0$, the limit of unit operator (C.3) implies the identity $G_{7 \mid \mathcal{O}_{i 3} \rightarrow \mathbb{1}} \equiv$ $\left(v_{23}^{6}\right)^{h_{3}-h_{6}}\left(v_{12}^{6}\right)^{h_{6}} G_{7}=G_{6}$.

From the vanishing components of $\boldsymbol{h}$ and $\boldsymbol{p}$, we have

$$
m_{2}=m_{12}=m_{22}=m_{24}=m_{33}=0 .
$$

As a result, we find that

$$
\begin{aligned}
F_{7} & =\frac{\left(-\bar{p}_{3}-h_{2}+d / 2-m_{3}\right)_{m_{3}}}{\left(p_{3}\right)_{-m_{1}}} \sum \frac{\left(-m_{3}\right)_{t_{2}}\left(\bar{p}_{3}-d / 2\right)_{t_{2}}}{t_{2} !\left(p_{3}-m_{1}\right)_{t_{2}}\left(p_{3}\right)_{t_{2}}\left(\bar{p}_{3}+h_{2}+1-d / 2\right)_{t_{2}}} \frac{\left(-m_{1}\right)_{r}\left(-m_{4}\right)_{r}\left(-p_{2}+d / 2-m_{1}\right)_{r}}{r !\left(1-p_{2}-h_{3}-m_{1}\right)_{r}\left(p_{4}-m_{1}\right)_{r}} \\
& =\frac{\left(-h_{2}-m_{1}^{\prime}\right)_{m_{1}^{\prime}}\left(p_{3}\right)_{m_{1}^{\prime}}}{\left(p_{3}\right)_{m_{1}^{\prime}-m_{2}^{\prime}}} F_{6 \mid \mathrm{comb} .}
\end{aligned}
$$

Thus, $G_{7 \mid \mathcal{O}_{i_{3}} \rightarrow \mathbb{1}}$ becomes

$$
\begin{aligned}
& G_{7 \mid \mathcal{O}_{i_{3}} \rightarrow \mathbb{1}}=\sum \frac{\left(p_{4}-m_{2}^{\prime}\right)_{m_{3}^{\prime}+m_{14}}\left(-h_{6}\right)_{m_{3}^{\prime}+m_{14}+m_{34}+m_{44}}\left(p_{4}-h_{3}+h_{6}\right)_{m_{3}^{\prime}+m_{11}+m_{13}}}{\left(p_{2}+h_{3}+m_{2}^{\prime}\right)_{-m_{3}^{\prime}}\left(p_{4}-h_{3}\right)_{2 m_{3}^{\prime}+m_{11}+m_{13}+m_{14}+m_{34}+m_{44}}\left(p_{4}-h_{3}+1-d / 2\right)_{m_{3}^{\prime}}} \\
& \times \frac{\left(p_{2}+h_{3}\right)_{m_{2}^{\prime}-m_{3}^{\prime}+m_{11}^{\prime}}\left(p_{3}\right)_{m_{1}^{\prime}}\left(p_{3}-h_{2}\right)_{m_{11}+m_{34}}\left(\bar{p}_{3}+h_{2}+h_{5}\right)_{m_{1}^{\prime}}}{\left(\bar{p}_{3}+h_{2}\right)_{2 m_{1}^{\prime}+m_{13}+m_{11}^{\prime}+m_{44}}\left(\bar{p}_{3}+h_{2}+1-d / 2\right)_{m_{1}^{\prime}}} \\
& \times \frac{\left(p_{2}+h_{2}\right)_{m_{1}^{\prime}+m_{2}^{\prime}+m_{13}+m_{11}^{\prime}+m_{44}}\left(-h_{2}-m_{1}^{\prime}\right)_{m_{1}^{\prime}}\left(-h_{2}\right)_{m_{1}^{\prime}-m_{2}^{\prime}+m_{11}+m_{34}}}{\left(p_{2}\right)_{2 m_{2}^{\prime}+m_{11}+m_{13}+m_{11}^{\prime}+m_{34}+m_{44}}\left(p_{2}+1-d / 2\right)_{m_{2}^{\prime}}} \\
& \times \frac{\left(-h_{3}\right)_{m_{2}^{\prime}+m_{3}^{\prime}+m_{11}+m_{13}+m_{34}+m_{44}}\left(-h_{5}\right)_{m_{2}^{\prime}+m_{13}+m_{11}^{\prime}+m_{44}}}{\left(p_{3}-h_{2}\right)_{m_{11}+m_{34}}} \\
& \times\left(\begin{array}{c}
m_{11} \\
k_{11}
\end{array}\right)\left(\begin{array}{c}
m_{13} \\
k_{13}
\end{array}\right)\left(\begin{array}{c}
m_{14} \\
k_{14}
\end{array}\right)\left(\begin{array}{c}
m_{34} \\
k_{34}
\end{array}\right)\left(\begin{array}{c}
m_{44} \\
k_{44}
\end{array}\right)\left(\begin{array}{c}
h_{6}-m_{3}^{\prime}-k_{14}-k_{34}-k_{44} \\
m_{12}^{\prime}
\end{array}\right)\left(\begin{array}{c}
k_{11} \\
m_{13}^{\prime}
\end{array}\right)\left(\begin{array}{c}
k_{44} \\
m_{22}^{\prime}
\end{array}\right) \\
& \times\left(\begin{array}{c}
h_{3}-h_{6}-m_{2}^{\prime}-k_{11}-k_{13}+k_{14} \\
m_{23}^{\prime}
\end{array}\right)\left(\begin{array}{c}
k_{13} \\
m_{33}^{\prime}
\end{array}\right)(-1)^{k_{11}+k_{13}+k_{14}+k_{34}+k_{44}+m_{12}^{\prime}+m_{13}^{\prime}+m_{22}^{\prime}+m_{23}^{\prime}+m_{33}^{\prime}} \\
& \times \frac{\left(1-v_{13}^{6}\right)^{m_{13}^{\prime}}}{m_{11} !} \frac{\left(1-v_{33}^{6}\right)^{m_{33}^{\prime}}}{m_{13} !} \frac{\left(1-v_{11}^{6}\right)^{m_{11}^{\prime}}}{m_{11}^{\prime} !} \frac{\left(1-v_{22}^{6}\right)^{m_{22}^{\prime}}}{m_{44} !} \frac{\left(1-v_{23}^{6}\right)^{m_{23}^{\prime}}}{m_{14} !} \frac{\left(1-v_{12}^{6}\right)^{m_{12}^{\prime}}}{m_{34} !} F_{6 \mid \mathrm{comb}} \prod_{1 \leq a \leq 3} \frac{\left(u_{a}^{6}\right)^{m_{a}^{\prime}}}{m_{a}^{\prime} !} \text {. }
\end{aligned}
$$

At this point, the resummations mirror the ones of the previous limit of unit operator. As such, they are left for the interested reader.

[1] S. Ferrara, A. F. Grillo, and R. Gatto, Tensor representations of conformal algebra and conformally covariant operator product expansion, Ann. Phys. (N.Y.) 76, 161 (1973); A. M. Polyakov, Nonhamiltonian approach to conformal quantum field theory, Zh. Eksp. Teor. Fiz. 66, 23 (1974) [Sov. Phys. JETP 39, 9 (1974)].

[2] F. A. Dolan and H. Osborn, Conformal partial waves and the operator product expansion, Nucl. Phys. B678, 491 (2004); Conformal partial waves: Further mathematical results, arXiv:1108.6194; P. Kravchuk, Casimir recursion relations for general conformal blocks, J. High Energy Phys. 02 (2018) 011.

[3] S. Ferrara and G. Parisi, Conformal covariant correlation functions, Nucl. Phys. B42, 281 (1972); S. Ferrara, A. F. Grillo, G. Parisi, and R. Gatto, The shadow operator formalism for conformal algebra. Vacuum expectation values and operator products, Lett. Nuovo Cimento 4, 115 (1972); D. Simmons-Duffin, Projectors, shadows, and conformal blocks, J. High Energy Phys. 04 (2014) 146. 
[4] D. Karateev, P. Kravchuk, and D. Simmons-Duffin, Weight shifting operators and conformal blocks, J. High Energy Phys. 02 (2018) 081; M. S. Costa and T. Hansen, AdS weight shifting operators, J. High Energy Phys. 09 (2018) 040.

[5] M. Isachenkov and V. Schomerus, Superintegrability of $d$-Dimensional Conformal Blocks, Phys. Rev. Lett. 117, 071602 (2016); V. Schomerus, E. Sobko, and M. Isachenkov, Harmony of spinning conformal blocks, J. High Energy Phys. 03 (2017) 085; V. Schomerus and E. Sobko, From spinning conformal blocks to matrix Calogero-Sutherland models, J. High Energy Phys. 04 (2018) 052; M. Isachenkov and V. Schomerus, Integrability of conformal blocks. Part I. Calogero-Sutherland scattering theory, J. High Energy Phys. 07 (2018) 180; I. Buri, V. Schomerus, and M. Isachenkov, Conformal group theory of tensor structures, J. High Energy Phys. 10 (2020) 004.

[6] E. Hijano, P. Kraus, E. Perlmutter, and R. Snively, Witten diagrams revisited: The AdS geometry of conformal blocks, J. High Energy Phys. 01 (2016) 146; M. Nishida and K. Tamaoka, Geodesic Witten diagrams with an external spinning field, Prog. Theor. Exp. Phys. 2017, $053 \mathrm{~B} 06$ (2017); A. Castro, E. Llabrs, and F. Rejon-Barrera, Geodesic diagrams, gravitational interactions \& OPE structures, J. High Energy Phys. 06 (2017) 099; E. Dyer, D. Z. Freedman, and J. Sully, Spinning geodesic Witten diagrams, J. High Energy Phys. 11 (2017) 060; H.-Y. Chen, E.-J. Kuo, and H. Kyono, Anatomy of geodesic Witten diagrams, J. High Energy Phys. 05 (2017) 070; C. Sleight and M. Taronna, Spinning Witten diagrams, J. High Energy Phys. 06 (2017) 100.

[7] S. Ferrara, A. F. Grillo, and R. Gatto, Manifestly conformal covariant operator-product expansion, Lett. Nuovo Cimento 2, 1363 (1971); S. Ferrara, R. Gatto, and A. F. Grillo, Conformal invariance on the light cone and canonical dimensions, Nucl. Phys. B34, 349 (1971); S. Ferrara, A. F. Grillo, and R. Gatto, Manifestly conformal-covariant expansion on the light 3cone, Phys. Rev. D 5, 3102 (1972); S. Ferrara, P. Gatto, and A. F. Grilla, Conformal algebra in spacetime and operator product expansion, Springer Tracts Mod. Phys. 67, 1 (1973); S. Ferrara, A. F. Grillo, G. Parisi, and R. Gatto, Covariant expansion of the conformal fourpoint function, Nucl. Phys. B49, 77 (1972); Erratum, Nucl. Phys. B53, 643 (1973); S. Ferrara, A. F. Grillo, R. Gatto, and G. Parisi, Analyticity properties and asymptotic expansions of conformal covariant green's functions, Nuovo Cimento A 19, 667 (1974); F. A. Dolan and H. Osborn, Conformal four point functions and the operator product expansion, Nucl. Phys. B599, 459 (2001); J.-F. Fortin and
W. Skiba, Conformal bootstrap in embedding space, Phys. Rev. D 93, 105047 (2016).

[8] J.-F. Fortin and W. Skiba, Conformal differential operator in embedding space and its applications, J. High Energy Phys. 07 (2019) 093; V. Comeau, J.-F. Fortin, and W. Skiba, Further results on a function relevant for conformal blocks, arXiv:1902.08598.

[9] J.-F. Fortin and W. Skiba, A recipe for conformal blocks, arXiv:1905.00036.

[10] J.-F. Fortin and W. Skiba, New methods for conformal correlation functions, J. High Energy Phys. 06 (2020) 028 .

[11] J.-F. Fortin, V. Prilepina, and W. Skiba, Conformal twopoint correlation functions from the operator product expansion, J. High Energy Phys. 04 (2020) 114; Conformal three-point correlation functions from the operator product expansion, arXiv:1907.08599; Conformal four-point correlation functions from the operator product expansion, J. High Energy Phys. 08 (2020) 115.

[12] J.-F. Fortin, W.-J. Ma, V. Prilepina, and W. Skiba, Efficient rules for all conformal blocks, arXiv:2002.09007.

[13] K. B. Alkalaev and V. A. Belavin, From global to heavylight: 5-point conformal blocks, J. High Energy Phys. 03 (2016) 184; V. Rosenhaus, Multipoint conformal blocks in the comb channel, J. High Energy Phys. 02 (2019) 142; V. Gonalves, R. Pereira, and X. Zhou, 20' Five-point function from $\mathrm{AdS}_{5} \times S^{5}$ supergravity, J. High Energy Phys. 10 (2019) 247; S. Parikh, Holographic dual of the five-point conformal block, J. High Energy Phys. 05 (2019) 051.

[14] C. B. Jepsen and S. Parikh, Propagator identities, holographic conformal blocks, and higher-point AdS diagrams, J. High Energy Phys. 10 (2019) 268.

[15] S. Parikh, A multipoint conformal block chain in $d$ dimensions, J. High Energy Phys. 05 (2020) 120.

[16] J.-F. Fortin, W. Ma, and W. Skiba, Higher-point conformal blocks in the comb channel, J. High Energy Phys. 07 (2020) 213.

[17] J.-F. Fortin, W.-J. Ma, and W. Skiba, Six-point conformal blocks in the snowflake channel, arXiv:2004.02824.

[18] T. Anous and F. M. Haehl, On the Virasoro six-point identity block and chaos, J. High Energy Phys. 08 (2020) 002 .

[19] H. Exton, Multiple hypergeometric functions and applications (Halstead Press, Ellis Horwood Limited, Chichester, 1976); H. M. Srivastava and P. W. Karlsson, Multiple Gaussian Hypergeometric Series (Halsted Press, Ellis Horwood Limited, Chichester, 1985). 